\title{
Studie zur Verwendung von Kinderschutzsystemen
}

Gerd Müller

Wolfgang Fastenmeier

Thomas Hummel 


\title{
Studie zur Verbesserung von Kinderschutzsystemen
}

\author{
Dr.-Ing. Gerd Müller \\ Prof. Dr. Wolfgang Fastenmeier \\ Dipl.-Ing. Thomas Hummel
}

Unfallforschung

der Versicherer 


\section{Impressum}

Gesamtverband der Deutschen Versicherungswirtschaft e. V.

Unfallforschung der Versicherer

Wilhelmstraße 43/43G, 10117 Berlin

Postfach 0802 64, 10002 Berlin

E-Mail: unfallforschung@gdv.de

Internet: www.udv.de

Facebook: www.facebook.com/unfallforschung

Twitter: @unfallforschung

YouTube: www.youtube.com/unfallforschung

ISBN-Nr.: 978-3-939163-85-5

Redaktion: Dipl.-Ing. Thomas Hummel

Bildnachweis: UDV und siehe Quellenangaben

Erschienen: 09/2018 


\section{Studie zur Verbesserung von Kinderschutzsystemen}

Bearbeitet durch:

Gesellschaft für Kraftfahrzeugtechnik Berlin - GKB UG

Dr.-Ing. Gerd Müller

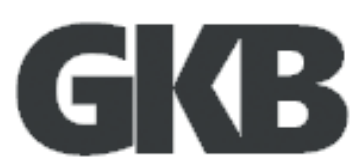

Mensch Verkehr Umwelt, Institut für Angewandte Psychologie Prof.-Dr. Wolfgang Fastenmeier

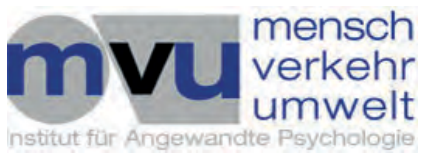

Bei der UDV betreut von:

Dipl.-Ing. Thomas Hummel

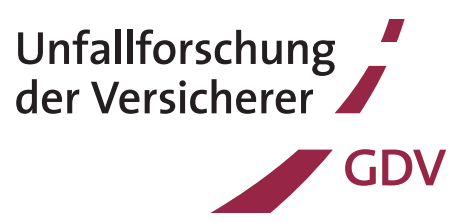





\section{Kurzfassung}

Ausgangslage.- In den letzten Jahren wurden zweifellos weitreichende Verbesserungen auf dem Gebiet der Kindersicherheit im Pkw erzielt. Die Einführung der gesetzlichen Sicherungspflicht im Jahr 1993 (Sicherung von Kindern in geeigneten Kinderschutzsystemen (KSS)) führte zudem zu einem erheblichen Anstieg der Sicherungsquote von Kindern in Pkw. Wie eine Reihe aufeinanderfolgender Studien seither gezeigt hat, besteht das Hauptproblem im Zusammenhang mit der Nutzung von Kindersitzen in deren falscher Benutzung (engl. Misuse). Rund zwei Drittel aller Kindersitze werden demnach fehlerhaft verwendet, was deren Schutzwirkung erheblich reduzieren oder ganz aufheben kann - ein Problem, das bei allen Modellen und in allen Systemgruppen auftritt. Ziel der vorliegenden Studie war es, den aktuellen Stand der Kindersicherheit im Pkw zu erheben und dabei insbesondere das Thema "Misuse“ ins Zentrum der Untersuchung zu stellen.

Felderhebungen zu Misuse.- Um über möglichst alle interessierenden Aspekte von Misuse Erkenntnisse zu erhalten, mussten sowohl Daten über die Häufigkeit, Schwere etc. von Misuse als auch über die zugrundeliegenden Kenntnisse, Einstellungen und Fertigkeiten der Nutzer erhoben werden. Deshalb wurde für die Feldstudie zu Misuse ein zweistufiges Beobachtungs- und Befragungskonzept erarbeitet. Im Zentrum der Beobachtungen an Kontaktplätzen stand zunächst die Erfassung von Misuse-Formen. Der Beobachtungsbogen umfasste vier Teile und zwar:

- Angaben zu Fahrzeug und Insassen,

- Angaben zur interviewten Person,

- Angaben zu Kind(ern), KSS und Misuse,

- Fragen zum festgestellten Misuse.

Für die Einstufung des erfassten Misuse wurde die schon in den Vorgängerprojekten verwendete Checkliste weiterentwickelt, in der die verschiedenen Misuse-Formen insbesondere nach Misuse-Schwere (leicht, mittel, schwer) systematisiert und mit Codes (z.B. „s-001“ für „schwerer Misuse, falsche Einbaurichtung“) versehen sind.

Der Erfassung von Kontextbedingungen, Handlungsmotiven und handlungsrelevanten Wissenselementen diente eine telefonische Nachbefragung. Der Fragebogen für die Nachbefragung umfasste ebenfalls vier Teile und zwar:

- Fragen zum Erwerb des Kindersitzes,

- Fragen zum Einbau des Kindersitzes (gilt in der Regel für KSS, bei denen Einbau und Sicherung zwei getrennte Vorgänge sind),

- Fragen zum Sichern im Kindersitz, 
- allgemeine Fragen zum Sichern im Kindersitz (handlungsrelevante Wissenselemente).

Ergebnisse.- Die in den Großräumen Berlin und München durchgeführte Feldstudie ist mit $\mathrm{n}=1.076$ erhobenen Fällen die mit Abstand größte Studie zur Kindersicherheit in Pkw und der Frage nach dem damit verbundenen Fehlgebrauch von KSS. Mit der Wahl unterschiedlicher Kontaktplätze wurde sichergestellt, dass verschiedene Fahrtzwecke (Einkauf, Bringen/Abholen vom Kindergarten, Schule, Freizeitfahrt) im Rahmen der Erhebungen streuten. Die Felderhebungen begannen im Juli 2016 und endeten im September 2017, womit eine Erhebung über alle Jahreszeiten und Wetterbedingungen gewährleistet war. Von den 1.076 erhobenen Fällen waren 1.046 Kinder in KSS gesichert, davon über $70 \%$ mit einem KSS + 3-Punkt-Gurt-Automatik-System sowie bereits mit knapp $30 \%$ erfreulicherweise in einem ISOFIX-System. Von den insgesamt 1.042 untersuchten Kindern waren $52 \%$ korrekt und 47,6 \% fehlerhaft gesichert. Damit liegt die Misusequote im Vergleich zu den bisherigen Studien erstmals unter $50 \%$. Die Misuse-Quote variiert signifikant in Abhängigkeit von den Systemarten. So erweist sich der Misuse-Anteil der Kindersitze der Klassen 0/0+ sowie 1 als überproportional hoch im Vergleich zur Klasse 2/3. Das bedeutet auch: nach wie vor sind es insbesondere die (sehr) jungen Kinder, die überdurchschnittlich oft von einer fehlerhaften Sicherung betroffen sind. ISOFIX-Systeme schneiden hochsignifikant besser ab als die herkömmlichen Befestigungsarten.

Eine bedeutsame Veränderung gegenüber den früheren Studien lässt sich auch hinsichtlich der Misuse-Schwere konstatieren, hier ist sogar ein gegenteiliger Effekt zu vermerken: War bislang eine Verschiebung von schwerem Misuse zu mittlerem und leichtem Misuse feststellbar, so zeigt sich in der aktuellen Studie ein erhöhter Anteil an schwerem Misuse, auf den etwa die Hälfte des gesamten Misuse entfällt gegenüber ca. $35 \%$ mittlerem und $15 \%$ leichtem Misuse. Erfreulich erscheint zwar, dass gängige Misuseformen durch moderne KSS eher vermieden werden, kommt es aber dennoch zu Fehlgebrauch, dann fällt dieser offenbar meist schwerwiegend aus.

Die Suche nach Misuse-Quellen verläuft weitgehend analog zu bekannten Erkenntnissen: Schlechte Wetterbedingungen wie Niederschlag und damit ggfs. verbundene Kleidungswechsel beim Kind sowie möglichst kurze Sicherungszeiten begünstigen Misuse ebenso wie kurze Fahrten unter 10 Minuten und den damit verbundenen Fahrtzwecken wie Holen/Bringen von Kita und Schule, kurze Erledigungen und Heimwege. Bemerkenswert erscheint: es sind insbesondere die jungen Eltern unter 30 Jahren sowie die Altersgruppe der 40-59-jährigen Sicherungsakteure, bei denen signifikant häufiger Misuse festgestellt wurde. Auch diejenigen, die die Schutzwirkung von KSS als sehr positiv beurteilen, begehen häufiger Misuse, wiegen sich also möglicherweise in trügerischer Sicherheit. Ausländische Nutzer aus dem südosteuropäischen Raum sowie dem Nahen Osten stellen nach wie vor 
eine besondere Problemgruppe bei der korrekten Sicherung von Kindern in Pkw dar, liegt doch deren Misuse-Quote sowie überhaupt die fehlende Sicherung deutlich höher als die deutscher Eltern.

Fehler beim Einbau ähneln sich in den Klassen 0/0+ sowie 1: Es dominieren Probleme, die im Zusammenhang mit dem Fahrzeuggurt stehen. Das sind insbesondere die Gurtlose, die Nichtbenutzung der Führungshilfen für den Gurt und das Vertauschen von Becken- und Schultergurt. In Klasse 2/3 treten relativ wenig Einbaufehler auf, hier herrschen Sicherungsfehler vor. Diese betreffen überwiegend Gurtlose des Beckengurtes sowie des Schultergurtes, verdrehte Fahrzeuggurte und weitere Fehler im Kontext der Gurte des KSS. In Gruppe 0/0+ sowie Gruppe1-Sitzen dominieren mit jeweils rund $90 \%$ die Gurtlose. Auch das Thema falsche Nutzung des Fangkörpers spielt in Gruppe 1-Sitzen eine Rolle.

Wie sich weiter zeigt, ist ein erheblicher Teil der Befragten der Auffassung, eine fehlerhafte Handhabung der Kindersitze hätte keinerlei Folgen für die Schutzwirkung der Systeme, nur ein geringer Teil äußerte eine realistische Einschätzung der damit verbundenen Risiken. Die meisten Eltern oder Sicherungsakteure antworteten allerdings mit „weiß nicht“. Hinsichtlich der subjektiven Begründungen für den festgestellten Misuse überwogen Antworten, die sich einerseits als unbewusste Nachlässigkeit interpretieren lassen. Andererseits zeigen sich Wissensdefizite in Bezug auf die korrekte Handhabung der Schutzsysteme, mangelnde/fehlerhafte Informationen zur korrekten Handhabung der Schutzsysteme, kurze Fahrtstrecke, Zeitdruck, der Wunsch nach Komfortverbesserung für das Kind, kleidungsbedingter Misuse, das Kind sichert sich selbst sowie „bewusste Nachlässigkeit“.

An der telefonischen Nachbefragung nahmen 107 Eltern teil. Vor dem Erwerb eines KSS wurden am häufigsten Testberichte in Zeitschriften sowie das Internet zu Rate gezogen; große Bedeutung wurde aber auch Ratschlägen von Verwandten/Bekannten beigemessen. Beratung im Fachgeschäft oder durch Automobilclubs u.ä. spielte demgegenüber eine etwas geringere, aber immer noch nennenswerte Rolle. Positive Testergebnisse, gefolgt von Kriterien wie Eignung für das Kind, Hersteller/Marke, einfache Handhabung, Komfort für das Kind sowie der Preis waren letztlich die ausschlaggebenden Gesichtspunkte beim Kauf des KSS.

Als Problembereiche erweisen sich einerseits die fragliche Handlungsrelevanz von Herstellerangaben, Platz- und Einbauprobleme bei mehreren Sitzen und 2-türigen Fahrzeugen. Ein Großteil der Befragten berichtet von Problemen beim Sichern, die meist mit durch von Kindern hervorgerufenen Schwierigkeiten (neben „Kind wehrt sich bei/gegen Sicherung“ betrifft das „Kind manipuliert unterwegs die Sicherung“, „Kind öffnet Sicherung während der Fahrt“) zusammenhängen. Darüber hinaus spielt die Handhabung der Gurte eine große Rolle. 
Insgesamt lässt sich hier festhalten: Die große Mehrheit der Befragten ist von der Schutzwirkung der Kindersitze überzeugt, weist also ein positives Sicherheitsbewusstsein auf. Trotz der z.T. guten Kenntnisse zu einigen der genannten Fragestellungen (Sicherungspflicht, ISOFIX) weisen die Befragten dennoch zu zentralen Fragen der korrekten Handhabung der KSS hinsichtlich Einbau und Sicherung z.T. deutliche Wissensdefizite auf.

Folgerungen.- Auf Basis der Untersuchungsergebnisse wurden verschiedene Maßnahmenvorschläge entwickelt. Diese richten sich an KSS- und Fahrzeughersteller, Gesetzgeber und Exekutive sowie Forschung und Verbraucherschutz. Zentrale Punkte betreffen u.a. die Förderung von ISOFIX, verbesserte Gebrauchsanleitungen und Piktogramme, Entwicklung von weiteren Kriterien für die Zulassungstests von KSS, Entwicklung verbindlicher Standards für die Durchführung von Fachberatungen. Dazu kommt die Weiterführung von Maßnahmen zur zielgruppenspezifischen Sensibilisierung und Aufklärung ausländischer Nutzer sowie der Nutzer im Allgemeinen. Obwohl die MisuseQuote bei KSS erstmals unter $50 \%$ gesunken ist, gehört deren fehlerhafte Handhabung nach wie vor zum festen Bestandteil ihrer Nutzung; zudem beeinflussen gesetzliche Änderungen sowie technische Neuerungen die alltägliche Nutzung von KSS. Deshalb erscheint es sinnvoll, Felduntersuchungen zu Misuse weiterhin in regelmäßigen Abständen vorzusehen. 


\begin{abstract}
Introduction.- Important advances have been made on children's transport safety in the last years (e.g. easier and more comfortable usage of child restraint systems (CRS), higher safety standards of CRS, homogeneous European rules like ECE-R44). Furthermore, the compulsory use of child safety seats in Germany since 1993 has considerably increased the rate of children secured by CRS or by seat belts. At the same time, however as results of a series of field observations reveal, a great number of CRS (about 60\%) has been persistently misused. Misuse impairs the protective capability of CRS and therefore can drastically reduce their protective performance. Therefore, an unalterable precondition for the protection of children as vehicle occupants is the proper and adequate use of CRS, that is a usage which doesn't affect or impair the protective capability of a safety seat. The purpose of the current study was to analyse the actual situation of children safety in cars with special focus on the misuse topic.
\end{abstract}

Field observations. - In order to completely cover all relevant aspects of misuse both data on the frequency, severity and types of misuse and the underlying knowledge, attitudes and skills of the users had to be recorded. Thus, a threefold concept of observation and inquiry was constructed. First of all misuse of CRS should be observed in public areas such as shopping centres, schools, road side restaurants etc. The observation form used included four parts with topics such as:

- Information about cars and passengers (e.g. model of car, number of doors, travel details, age and number of passengers/children, seating positions).

- Socio-demographical information about the person to be interviewed (e.g. gender, age, nationality).

- Information about the children, CRS and misuse (e.g. seating position, type of CRS, type and severity of misuse).

- Questions concerning the observed misuse (e.g. who did the installation / securing, is misuse affecting the safety of the child, possible reasons for misuse).

The form which was used to classify the misuse configurations was based on the approved form already used in previous studies, but was enhanced further on. The form systematically lists all deviations from the intended application and use of CRS according to general errors and mistakes, incorrect use concerning both installation of the CRS and securing of the child in the seat, and takes into account system-specific (following the ECE group classification) mistakes. The mistakes are defined by codes which include an assessment of the severity of misuse (slight, medium, severe). 
Subsequently (by telephone interview) a questionnaire was used to get more information about the misuse-relevant situational context, motives and knowledge of the users. The questionnaire had four sections as follows:

- Information about the acquisition of the CRS (e.g. when and where; is CRS new, second hand, borrowed? information sources).

- Questions concerning the installation of CRS (e.g. objective and subjective criteria for correct installation; type of problems; possible reasons for problems)

- Questions concerning the securing of the child (e.g. objective and subjective criteria for correct securing; type of problems, possible reasons for problems)

- General questions concerning protection-relevant knowledge of the users (e.g. safety belt regulations, confidence into the safety effects of CRS, airbags and CRS).

Results.- The field observations were conducted in Greater Berlin and Greater Munich and comprised $n=1,076$ cases which makes this study the largest sample ever. All relevant driving purposes (shopping, leisure activities, kindergarten, school) and all relevant weather conditions from July 2016 to September 2017 were included in the observations. In 1,046 cases the children had been secured in CRS, about $30 \%$ thereof in ISOFIX-systems. The misuse rate was $47 \%$, i.e. the first time ever below $50 \%$. Misuse rates depend significantly on the type of CRS: Higher misuse rates for group 0/0+ and group $1 \mathrm{CRS}$, typically where installing and securing are two separate actions, lower misuse rates for group $2 / 3 \mathrm{CRS}$. This indicates that especially younger children are in danger not to be secured properly. ISOFIXsystems are significantly better than all other types of CRS.

One negative effect compared to the previous studies is that misuse severity is increased. Misuse with heavy or severe impact on the protective capability was most likely to occur to Rearward Facing Systems like car beds and least probably/likely to booster seats and booster cushions, respectively.

As far as misuse influencing factors are concerned the results are in analogy to the previous studies: bad weather conditions, change of clothes, short trips < 10 minutes and related driving purposes such as driving to kindergarten and school, short transactions and going home lead to significantly higher misuse rates. It's also interesting that both parents and users with an age lower 30 years and users with an age of 40-59 years are involved in higher misuse rates. Moreover, research indicates evidence that especially migrants can be labelled as problem groups, because both the compliance to safety belt regulations and the misuse rate is significantly worse than that of German citizens.

The most frequent types of misuse both in installation and securing were loosely anchored CRS, loose straps, incorrect belt routing, and belt routing crossing the neck. This holds 
especially true for group $0 / 0+$ and group 1 -systems. In group 2/3-systems securing problems such as loose straps of pelvis and shoulder straps are dominating. In cases of misuse most of the adults questioned were convinced that their usage of the CRS was correct.

The telephone interview was conducted with 107 parents and users. About one in four adults were only partly or not at all convinced of the protective capabilities provided by a CRS. Before purchasing a CRS the main information sources were test reports and Internet recommendations, followed by advice from friends/relatives and consulting of professionals and automobile clubs. In the end criteria such as positive test reports, CRS-brand, child comfort, price and good usability were the crucial factors. Main problem areas concern too complicated instructions, user manuals and pictograms, installation problems using more than one seat and installing CRS in smaller cars (2-3 doors). Moreover, problems generated by the children themselves are dominant: child refuses, child manipulates or opens the clips during the trip.

Summing up it can be stated that both the majority of the users are convinced of the protective effects of CRS and the sensibility for safety issues is enhanced. Despite comparatively improved knowledge about topics such as safety belt regulation or new systems (ISOFIX) a relevant part of the users reveals unsafe behaviour due to poor knowledge or poor skills. Finally, we should learn that not only parents and adult users of CRS are target groups, but also the children themselves, as they are an active part in CRSuse and have their own subjective needs and attitudes towards being secured in CRS.

Conclusions.- Finally recommendations for the improvement of child safety in cars are developed; they are addressed to car and CRS-manufacturers, authorities, traffic safety councils and research. Crucial points are related to improved manuals and pictograms, criteria for the homologation of CRS including usability aspects, better standards for qualified information of specialized dealers. Moreover, target group measures both for the general public and migrants have to be further developed. Although for the first time ever the misuse ratio of CRS is lower than $50 \%$, the incorrect use of safety seats is still prevalent. As both legal rules and technical improvements have an influence on user behavior it seems to be appropriate to continue field observations on misuse of CRS regularly. 


\section{Inhaltsverzeichnis:}

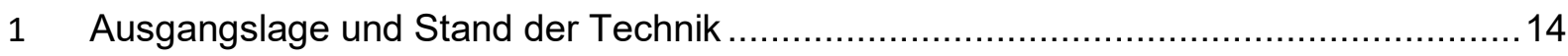

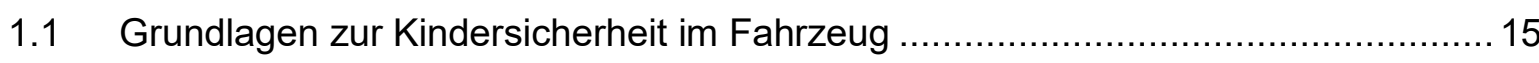

1.2 Bisherige Untersuchungen zum Fehlgebrauch von Kindersitzen.........................21

1.3 Bisherige Maßnahmen zur Vermeidung von Misuse .................................... 25

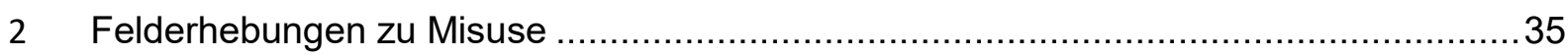

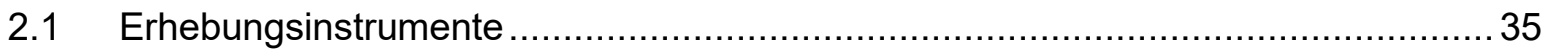

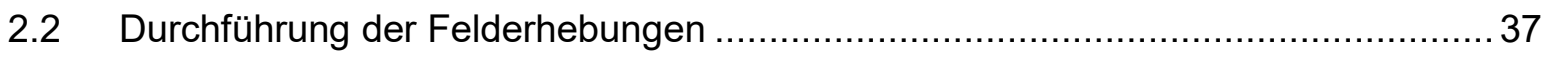

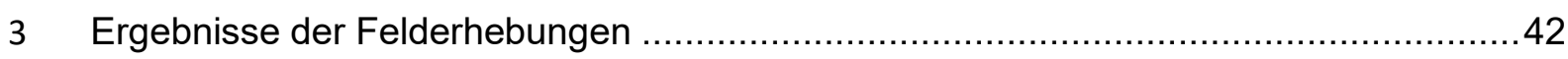

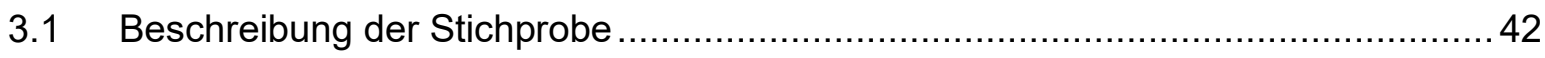

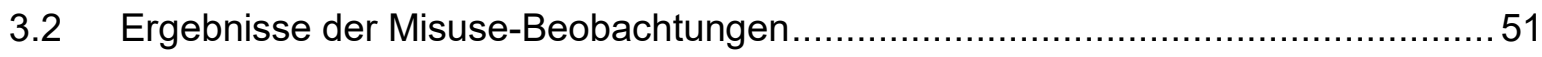

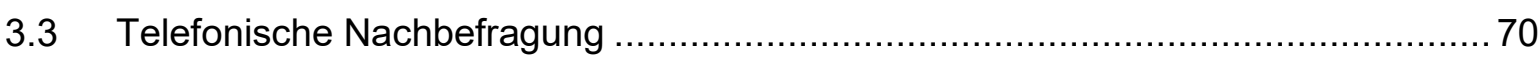

$4 \quad$ Mögliche Maßnahmen / Verbesserungsvorschläge und Empfehlungen......................80

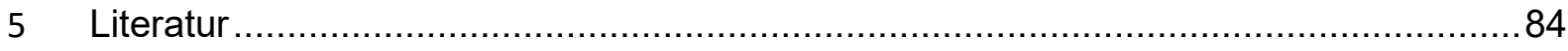

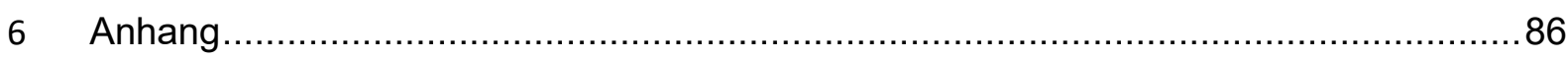

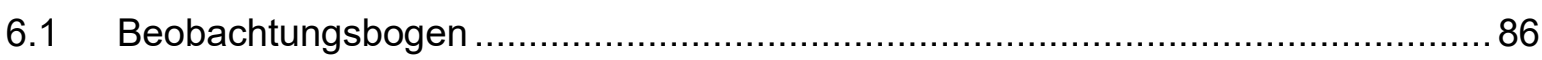

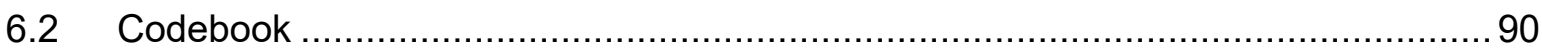

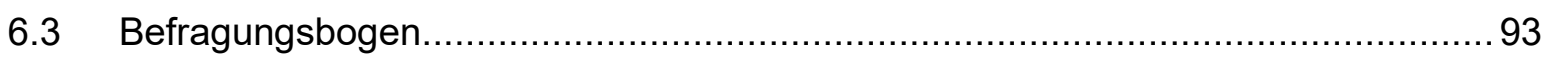

6.4 Struktur des Testprotokolls von EuroNCAP zur Kindersicherheit ...................... 102

6.5 Weitere Auswertung der Feldstudie ........................................................ 107

6.6 Ergebnisse der Schlittenversuche zur Darstellung der Folgen von Misuse........... 122 


\section{Ausgangslage und Stand der Technik}

In Zeiten eines hohen und sehr individuellen Bedürfnisses nach Mobilität ist die Nutzung des Pkws auch zur Beförderung von Kindern selbstverständlich. Da die Schutzeinrichtungen im Fahrzeug für erwachsene Insassen ausgelegt sind, müssen für Kinder spezielle Schutzeinrichtungen, sogenannte Kinderschutzsysteme (KSS), verwendet werden. Je nach Alter, Gewicht und Größe der Kinder gibt es bestimmte Körperregionen, die im Fall eines Unfalls besonders gefährdet sind, entsprechend gibt es verschiedene Schutzsysteme, die in Abhängigkeit vom Gewicht des Kindes zu verwenden sind.

Die Schutzwirkung von Kindersitzen hat sich seit der gesetzlichen Einführung ihrer Nutzungspflicht im Jahr 1993 stetig verbessert. Obwohl im Handel eine große Bandbreite an verschiedenen Modellen angeboten wird, was sich nicht zuletzt auch im Preis der Kindersitze zeigt, kann davon gesprochen werden, dass selbst die einfachsten und billigsten Sitze ein ausreichendes Maß an Schutz bieten. Das Hauptproblem im Zusammenhang mit der Nutzung von Kindersitzen besteht in deren falscher Benutzung (engl. Misuse). Studien dazu haben immer wieder gezeigt, dass rund zwei Drittel aller Kindersitze fehlerhaft verwendet werden, was deren Schutzwirkung erheblich reduzieren oder ganz aufheben kann. Auch wenn es hinsichtlich der Fehleranfälligkeit zwischen guten und schlechten Kindersitzen erhebliche Unterschiede gibt, muss festgestellt werden, dass das Problem der Fehlbenutzung bei allen Modellen und in allen Systemgruppen auftritt.

Der Fehlgebrauch von Kindersitzen und die damit einhergehenden Probleme wurden bereits vor längerer Zeit als grundlegendes Thema im Bereich der Kindersicherheit im Fahrzeug erkannt und untersucht. Dabei geht es zum einen darum, den Ist-Stand anhand von Feldstudien zu erheben und den Fehlgebrauch zu quantifizieren und zu analysieren, zum anderen wird versucht, aus verschiedenen Blickwinkeln die Gründe für den Misuse zu ergründen und mögliche Gegenmaßnahmen zu erarbeiten. Im Rahmen dieser Forschungsarbeiten ist es immer wieder nötig, die reale Situation zu erfassen, um Veränderungen im Sicherungsverhalten festzustellen. Dadurch können bisherige Maßnahmen auf ihren Erfolg hin überprüft und bewertet werden. Gleichzeitig lassen sich daraus Erkenntnisse für weiterführende Schritte ableiten.

Daraus ergibt sich die Notwendigkeit, solche Feldstudien in regelmäßigen Abständen zu wiederholen. Seit der letzten großen Feldstudie, die u.a. in Deutschland und auch bereits vor fünf Jahren durchgeführt wurde [Casper, 2012], haben sich grundlegende Randbedingungen geändert. Das ISOFIX-System als standardisierte Anbindung zwischen Fahrzeug und Kindersitz ist fahrzeugseitig mittlerweile gesetzlich vorgeschrieben, außerdem ist mit der ECE-R 129 eine neue Gesetzgebung zur Zulassung von Kindersitzen in Kraft getreten. Auch werden die sogenannten Fangkörpersysteme in den letzten Jahren wieder vermehrt nachgefragt, auch wenn ihre Schutzwirkung in Fachkreisen umstritten ist. 
In diesem Sinne ist es Aufgabe und Ziel der hier vorliegenden Misusestudie, einerseits ein aktuelles Bild von der Nutzungssituation von Kindersitzen zu erhalten. Anderseits kann damit überprüft werden, ob in der Vergangenheit eingeschlagene Wege zur Verbesserung der Kindersicherheit im Pkw zielführend waren. Technische und nutzungsspezifische Ursachen für Misuse können gezielt untersucht werden. Hinsichtlich des Umfangs setzt diese Studie neue Maßstäbe. Mit dem angestrebten Ziel von etwa 1.000 erhobenen Fällen ist diese Erhebung deutlich umfangreicher als alle bisher durchgeführten Feldstudien zu diesem Thema. Das erlaubt auch gezielte statistische Analysen zu sehr spezifischen Einzelfragen.

\subsection{Grundlagen zur Kindersicherheit im Fahrzeug}

Um Kinder im Fahrzeug im Fall eines Unfalls optimal zu schützen, müssen sie in einem geeigneten Sicherungssystem, einem Kindersitz, befördert werden. Das ist einerseits der Tatsache geschuldet, dass alle im Fahrzeug vorhandenen Rückhalteeinrichtungen für erwachsene Insassen ausgelegt sind und für kleine Insassen ihre Schutzwirkung nicht entfalten können. So ist beispielsweise die gesamte Gurtgeometrie nicht dafür ausgelegt, Menschen unter einer bestimmten Körpergröße an der Fahrzeugverzögerung teilhaben zu lassen. Andererseits gibt es grundsätzliche anatomische Unterschiede zwischen Kindern und Erwachsenen, die je nach Alter des Kindes auf verschiedene Weise berücksichtigt werden müssen. Selbst wenn es also möglich wäre, die fahrzeugseitigen Rückhaltesysteme auf den entsprechenden Größenbereich für Kinder einzustellen, wäre damit dennoch kein geeignetes Rückhaltesystem geschaffen. Das im Zusammenhang mit der Fürsorge für Kinder häufig erwähnte Zitat „Kinder sind keine kleinen Erwachsenen“ ist auch hier sehr treffend. Wesentliche Unterschiede im Körperbau von Kindern verschiedener Altersgruppen und von Erwachsenen sind in Fehler! Verweisquelle konnte nicht gefunden werden. argestellt. 


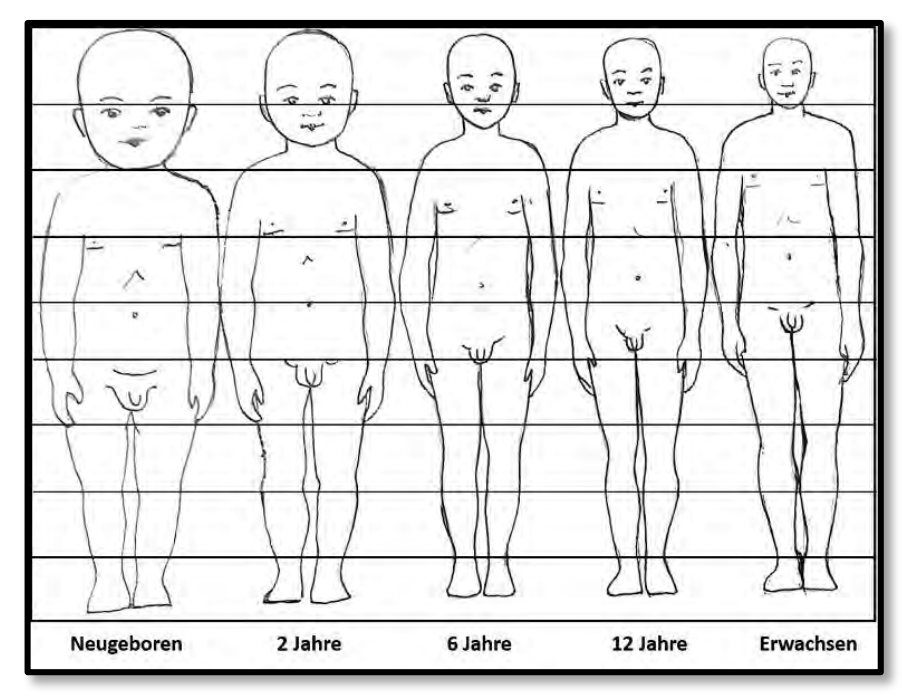

Abbildung 1: Vergleich der Körperproportionen in unterschiedlichen Entwicklungsstufen

Menschen verschiedener Altersgruppen sind hier auf eine Einheitsgröße skaliert. Gut zu sehen ist, dass der Kopf bei einem Neugeborenen rund ein Viertel seiner Gesamtgröße ausmacht, während er bei einem Erwachsenen nur rund einem Achtel der Gesamtgröße entspricht. Ebenso verhält es sich mit dem Gewicht des Kopfes. Bei einem Neugeborenen wiegt dieser knapp ein Kilo und macht damit rund ein Viertel des Gesamtgewichts aus. Im Gegensatz dazu trägt das Kopfgewicht eines durchschnittlichen erwachsenen Mannes nur etwa sechs Prozent zum Gesamtgewicht bei. Der schwere und große Kopf eines Babys und die gleichzeitig gering ausgeprägte Halsmuskulatur bedeuten, dass der Halsbereich einen besonderen Schutz vor äußeren Belastungen benötigt.

Im Brustbereich profitieren Kinder zunächst von relativ elastischen Strukturen der Rippen. Diese können ohne zu brechen Kräfte aufnehmen und so die inneren Organe schützen. Überschreiten diese Kräfte jedoch ein bestimmtes Maß, können die Rippen keinen wirkungsvollen Schutz mehr bieten und leiten die Kräfte direkt nach innen weiter. Darüber hinaus liegt bei kleinen Kindern eine sehr hohe Dichte der inneren Organe vor, insbesondere die Lungen nehmen im Verhältnis zum Gesamtkörper ein großes Volumen ein und drücken stark nach außen, wodurch die Verletzungsgefahr durch äußere Krafteinwirkungen sehr groß ist. Entsprechend muss der Thoraxbereich durch geeignete Maßnahmen seitens des Kindersitzes geschützt werden.

Der Abdominalbereich ist bei Erwachsenen und Kindern gleichermaßen ungeschützt vor Krafteinwirkungen. Es gibt keine knöcherne Struktur, die einen geeigneten Schutz für die Organe liefert. Während die Leber bei Erwachsenen fast komplett hinter den Rippen liegt, ist das bei Kindern nicht der Fall, weiterhin haben Erwachsene eine stärkere Bauchmuskulatur. 
Bei Kindern kommt hinzu, dass die Organe des Verdauungstrakts ebenfalls ein vergleichsweise großes Volumen einnehmen und somit weniger Möglichkeiten zum „Ausweichen“ bei möglichen Intrusionen haben.

Bei erwachsenen Insassen kann der Abdominalbereich vor Krafteinleitungen seitens des Fahrzeuggurts durch das Becken geschützt werden. Dieses ist robust genug um große Kräfte aufzunehmen, gleichzeitig bietet es aufgrund seiner Form einen guten Anlagepunkt für den Fahrzeuggurt. Die Beckenknochen eines Kindes sind dafür nicht geeignet. Zwar sind sie ebenfalls robust genug um Kräfte aufzunehmen, allerdings bieten sie aufgrund der noch wenig ausgebildeten Beckenschaufeln dem Fahrzeuggurt keinen guten Halt. Es besteht die Gefahr, dass der Gurt nach oben in den Abdomen rutscht und dort erhebliche Verletzungen verursacht.

Aus den genannten Gründen ist es notwendig, Kinder altersgemäß in geeigneten Kindersitzen zu befördern. Diese werden entsprechend der Zulassungsvorschrift von Kindersitzen (ECE-R 44) in verschiedene Gruppen in Abhängigkeit vom Gewicht des Kindes eingeteilt (Tabelle 1).

\begin{tabular}{|c|c|l|}
\hline Gruppe & Gewicht & \multicolumn{1}{|c|}{ Bemerkung } \\
\hline 0 & bis $10 \mathrm{~kg}$ & $\begin{array}{l}\text { rückwärtsgerichtet (Babyschale), eigenes } \\
\text { Gurtsystem } \\
\text { bis etwa 12 Monate }\end{array}$ \\
\hline $0+$ & bis 13 kg & $\begin{array}{l}\text { rückwärtsgerichtet (Babyschale), eigenes } \\
\text { Gurtsystem (diese Gruppe wurde nachträglich } \\
\text { eingeführt) } \\
\text { bis etwa 18 Monate }\end{array}$ \\
\hline 1 & $9-18 \mathrm{~kg}$ & $\begin{array}{l}\text { i.d.R. vorwärtsgerichtet, eigenes Gurtsystem } \\
\text { oder Fangkörper } \\
\text { ab etwa 15 Monaten, bis 3,5-4 Jahre }\end{array}$ \\
\hline 2 & $15-25 \mathrm{~kg}$ & $\begin{array}{l}\text { fast immer in Verbindung mit Gruppe 3, kein } \\
\text { eigenes Gurtsystem } \\
\text { ab etwa 4 Jahre }\end{array}$ \\
\hline 3 & $22-36 \mathrm{~kg}$ & $\begin{array}{l}\text { fast immer in Verbindung mit Gruppe 2, kein } \\
\text { eigenes Gurtsystem } \\
\text { ab etwa 7 Jahre }\end{array}$ \\
\hline
\end{tabular}

Tabelle 1: Gruppeneinteilung von Kindersitzen gemäß ECE-R 44 
Dabei sind Kindersitze vorstellbar, die für mehrere oder alle Gruppen geeignet sind, in der Praxis haben sich allerdings hauptsächlich drei Gruppen durchgesetzt: Gruppe 0+, Gruppe 1 , Gruppe 2/3.

\section{Gruppe 0/0+}

Sitze der Gruppe 0/0+ werden auch als Babyschalen bezeichnet und müssen immer rückwärtsgerichtet im Fahrzeug installiert werden (Abbildung 2). Dadurch ist sichergestellt, dass es beim Frontalaufprall, der häufigsten Aufprallart, zwischen Kopf und Oberkörper des Kindes keine Relativbewegung gibt, die zu großen Belastungen am Hals führen könnte. Sitze der Gruppe 0/0+ sind mit einem eigenen Drei-Punkt-Gurtsystem (Y-Gurt) oder 5Punkt-Gurtsystem ausgestattet, welches derart gestaltet ist, dass Kräfte nur an der stabilen Knochenstruktur eingeleitet werden (Schlüsselbein, Beckenknochen) und das Kind somit bestmöglich vor inneren Verletzungen geschützt wird.

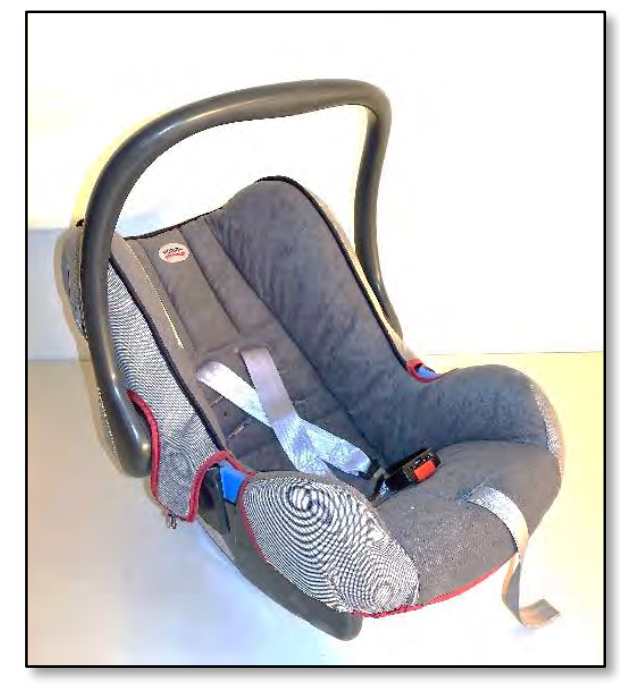

Abbildung 2: Gruppe 0/0+ Sitz

Bedeutende Fehler, die bei Babyschalen zu beobachten sind:

- falsche Einbaurichtung

- Vertauschen von Becken- und Schultergurt des Fahrzeuggurts.

Die falsche Einbaurichtung führt zum einen dazu, dass die Babyschale nicht fest mit dem Fahrzeuggurt verbunden werden kann, da dazu die Gurtführung am Kindersitz nicht geeignet ist. Vor allem aber kann die Schale ihre eigentliche Funktionsweise, nämlich das gleichmäßige Abstützen von Oberkörper und Kopf, nicht erfüllen. Im Fall einer 
Frontalkollision kann es durch die starke Vorverlagerung des Kopfes zu erheblichen Verletzungen im Halsbereich des Kindes kommen.

Das Vertauschen von Becken- und Schultergurt führt dazu, dass die Babyschale nahezu nicht mit dem Fahrzeug verbunden ist. Bei einer Frontalkollision bewegt sich die Schale unkontrolliert durchs Fahrzeug und wird durch den Fahrzeuggurt kaum zurückgehalten. Dadurch können ebenfalls erhebliche Verletzungen entstehen (siehe dazu auch Kapitel 6.6).

\section{Gruppe 1}

Sitze der Gruppe 1 werden in den meisten Fällen vorwärtsgerichtet im Fahrzeug eingebaut und verfügen ebenfalls über ein eigenes Gurtsystem. Eine Ausnahme bilden dabei die sogenannten Fangkörpersysteme; diese haben anstelle eines Gurtsystems einen Fangkörper, der das Kind zurückhält (Abbildung 3). Während hier keine besonderen Schutzmaßnahmen für den Halsbereich vorgesehen sind, besteht ein wesentliches Konstruktionsprinzip dieses Sitzes ebenfalls in der Vermeidung von Krafteinleitung in kritische Körperregionen. Durch den Fünf-Punkt-Gurt werden auch hier die Kräfte primär in die Schulterknochen bzw. ins Becken eingeleitet, Abdomen und Thorax sind weitestgehend geschützt bzw. durch eine großflächige Krafteinleitung wenig belastet.
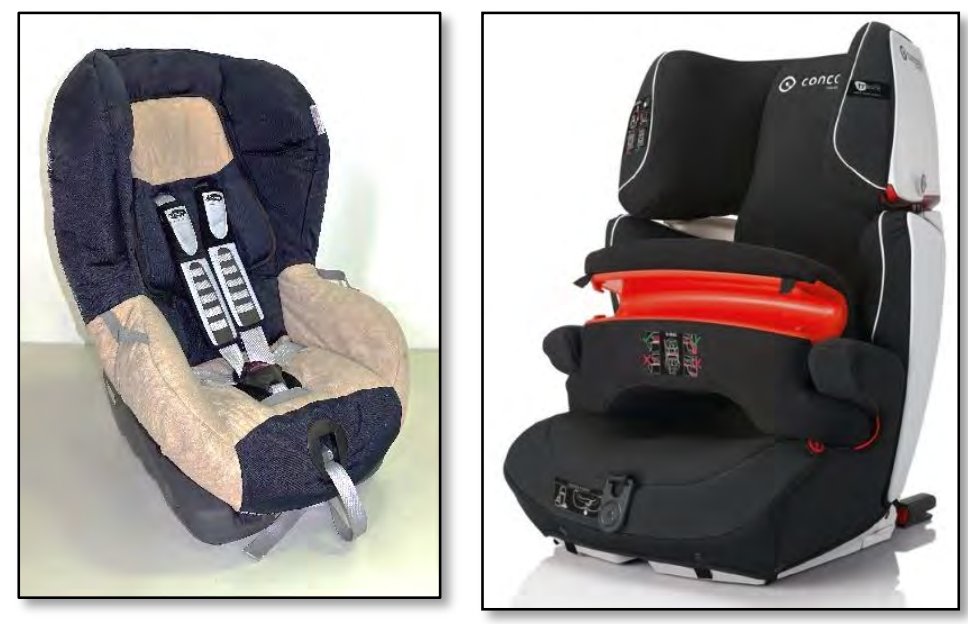

Abbildung 3: Gruppe 1 Sitz mit Fünf-Punkt-Gurtsystem (links) und mit Fangkörpersystem (rechts)

Bedeutende Fehler, die bei Gruppe 1 Sitzen zu beobachten sind:

- Kind ist zu klein für diese Sitzgruppe

- Die Führung des Fahrzeuggurts ist falsch

- Gurtlose im KSS oder im Fahrzeuggurt 
Dass Kinder zu früh in einen Gruppe 1 Sitz wechseln, ist ein häufig festzustellender Fehler. Im Ergebnis dessen werden die oben beschriebenen Schutzvorteile einer Babyschale, das gleichmäßige Abstützen von Kopf- und Oberkörper, aufgehoben. Das führt dazu, dass bei einer Kollision Verletzungen im Halsbereich sehr wahrscheinlich sind. Diese können schon bei mittlerer Unfallschwere erheblich sein.

Die korrekte Führung des Fahrzeuggurts erweist sich immer wieder als große Herausforderung. Werden dabei Fehler gemacht, ist von einer leichten Gurtlose bis zu einem völlig ungesicherten Sitz jedes Szenario vorstellbar, was im Ergebnis dazu führen kann, dass sich der Kindersitz unkontrolliert durchs Fahrzeug bewegt und somit Verletzungen beim Kind und auch bei anderen Insassen wahrscheinlich macht.

Eine Gurtlose im Gurtsystem des Kindersitzes oder im Fahrzeuggurt führt zu einer unerwünschten Vorverlagerung des Kindes, welche zu einem Kopfkontakt mit Fahrzeugteilen führen kann. Damit steigt das Risiko für Kopfverletzungen.

\section{Gruppe 2/3}

Sitze dieser Gruppe verfügen über kein eigenes Gurtsystem. Hier werden Kind und Kindersitz gemeinsam mit dem Fahrzeuggurt gesichert. Sitze dieser Gruppe gibt es entweder als einfache Sitzerhöhung oder als Sitzerhöhung mit Rückenlehne (Abbildung 4). Beide Systeme haben zunächst die Aufgabe, den Fahrzeuggurt so zu führen, dass er, wie in den zuvor beschriebenen Sitzen, an geeigneten Körperstellen Kräfte einleitet. Entsprechend sind diese Sitze mit Gurtführungen im Beckenbereich versehen, die ein Hochrutschen des Gurtes in den Abdominalbereich verhindern sollen. Gleichzeitig sorgt die erhöhte Sitzposition dafür, dass der Schultergurt über die Schulter und nicht über den Hals des Kindes verläuft. Ist der Sitz mit einer Rückenlehne versehen, so befindet sich im Schulterbereich ein zusätzlicher Gurtführungspunkt, der den gewünschten Verlauf über die Schulter sicherstellt. Außerdem sorgen seitliche Kopfpolster für einen Schutz im Fall eines Seitenaufpralls.
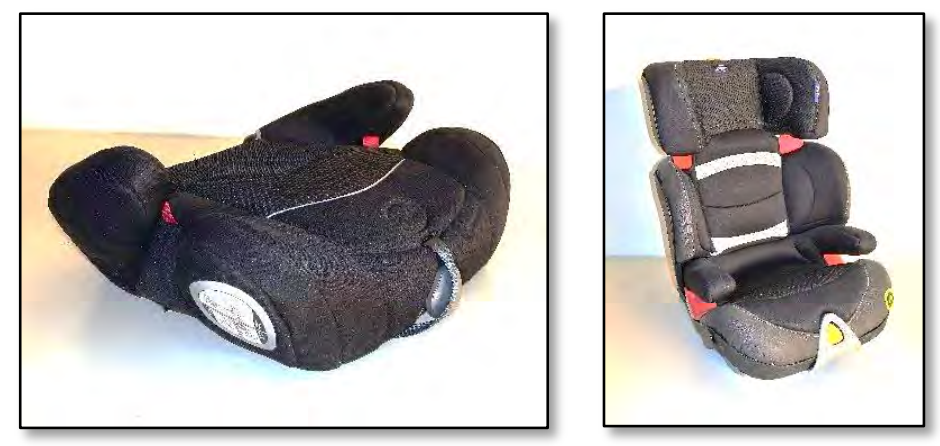

Abbildung 4: Gruppe 2/3 Sitz ohne und mit Rückenlehne 
Bedeutende Fehler, die bei Gruppe 2/3 Sitzen zu beobachten sind:

- Kind ist zu klein für diesen Kindersitz

- Falsche Führung des Fahrzeuggurts

Wie bei Gruppe 1 Kindersitzen kommt es auch in dieser Gruppe häufig vor, dass die Kinder für diese Sitze noch zu klein sind. Der Sitz kann innen dann keine optimale Sicherheit bieten, gleichzeitig lässt sich die Gurtführung nicht auf ihre Größe anpassen. Im Ergebnis ist damit zu rechnen, dass der Gurt selbst beim Kind Verletzungen verursacht oder aber das Kind nicht zurückhalten kann.

Die falsche Führung des Fahrzeuggurts kann je nach Ausführung unterschiedliche Folgen haben. Wird der Gurt beispielsweise nicht durch die entsprechenden Gurtführungen im Beckenbereich geleitet, kann dieser im Fall einer Frontalkollision in den Abdominalbereich einschneiden und dort erhebliche Verletzungen verursachen. Ebenfalls häufiger zu beobachten ist die Führung des Schultergurts unterhalb des Arms des Kindes. Diese vermeintliche Komforterhöhung führt dazu, dass der Oberkörper des Kindes nicht zurückgehalten wird, womit es zu einer großen Vorverlagerung kommt, Kopfverletzungen sind wahrscheinlich. Gleichzeitig rutscht der Schultergurt nach unten und es kann ebenfalls zu Verletzungen im Bauchbereich kommen.

In der neuen Gesetzgebung zur Zulassung von Kindersitzen, der ECE-R 129, ist diese starre Einteilung der Kindersitzklassen nicht mehr vorgesehen. Vielmehr obliegt es dem Kindersitzhersteller selbst eine Einteilung seiner Modelle vorzunehmen. Ob sich diese Einteilung nach der Größe, dem Alter oder dem Gewicht des Kindes richtet, bleibt ebenfalls dem KSS-Hersteller überlassen. Wesentliche Randbedingung seitens des Gesetzgebers ist jedoch, dass sichergestellt werden muss, dass Kinder bis zur Vollendung des 15. Lebensmonats nur rückwärtsgerichtet befördert werden.

\subsection{Bisherige Untersuchungen zum Fehlgebrauch von Kindersitzen}

In den letzten 25 Jahren gab es zahlreiche mehr oder weniger umfangreiche Untersuchungen zum Fehlgebrauch von Kindersitzen. Die erste große Studie vom GDV, beauftragt durch die Bundesanstalt für Straßenwesen (BASt), aus dem Jahr 1997 [Langwieder, 1997], bildete dabei sicherlich die Grundlage für weitere Forschungen. In Feldstudien wurde immer wieder untersucht, wie das reale Sicherungsverhalten aussieht und welche Entwicklungen im Verlauf mehrerer Jahre dabei zu beobachten sind. Sie sind damit ein wichtiges Instrument, um zu überprüfen, inwiefern sich Neuerungen im Markt 
durchsetzen und ob sie ggf. die erwünschten Resultate erzielen. Die wesentlichen in Deutschland durchgeführten Feldstudien werden im Folgenden vorgestellt.

\subsubsection{Ergebnisse bisheriger Feldstudien}

Eine wichtige Basis für Untersuchungen rund um den Fehlgebrauch von Kindersitzen stellen Feldstudien dar. Sie ermöglichen einen Blick auf das reale Sicherungsverhalten von Kindern im Pkw und beschreiben so die tatsächliche Ist-Situation. Werden diese Feldstudien regelmäßig wiederholt, kann neben dem aktuellen Status auch eine zeitliche Entwicklung im Vergleich der einzelnen Studien analysiert werden. Das kann Veränderungen bei der Sicherungsqualität ebenso wie die Verbreitung bestimmter Sitze oder Sicherungstechniken betreffen.

Im Regelfall werden neben der reinen Beobachtung auch kurze Befragungen mit den beteiligten Personen durchgeführt. Dadurch lassen sich verhaltensspezifische Faktoren ermitteln, die möglicherweise mit ursächlich für den Fehlgebrauch sind. Auch ergibt sich durch mehrere Studien in Folge die Möglichkeit zu untersuchen, inwiefern Aufklärungskampagnen oder andere Maßnahmen zur Reduzierung von Misuse Erfolg haben und ggf. das Problembewusstsein der Anwender verändert haben.

Die erste umfassende Feldstudie in Deutschland zur Sicherungssituation von Kindern im Pkw wurde 1995 erhoben und 1997 veröffentlicht [Langwieder, 1997]. Die Feldstudie ist dabei ein Teil einer insgesamt umfassenden Untersuchung zur Verbesserung des Schutzes von Kindern in Pkw. Für die Feldstudie wurde ein Codebook erarbeitet, in welchem nahezu alle Fehler und ihre Ausprägungsformen (leicht, mittel, schwer) codiert sind. Dieses Codebook fand auch in weiteren Studien Verwendung und wurde nicht zuletzt auch für die hier vorgestellte Studie in modifizierter Form verwendet, sodass eine gute Vergleichbarkeit der einzelnen Ergebnisse gegeben ist. Im weiteren Verlauf wurden im Jahr 2003 [Hummel, 2003], im Jahr 2006 [Fastenmeier, 2006], im Jahr 2008 [Hummel, 2008] und im Jahr 2012 [Casper, 2012] weitere Untersuchungen veröffentlicht.

Als Kernpunkt dieser Studien kann sicherlich die Frage nach der Häufigkeit des festgestellten Misuse gelten. Ein Vergleich der fünf genannten Studien macht deutlich, dass es im Zeitraum von über 15 Jahren keine signifikanten Veränderungen bei der Häufigkeit von Fehlbedienungen gab (Abbildung 5 ). Eine korrekte Sicherung konnte bei rund einem Drittel der Kinder, die einen Kindersitz benutzt haben, festgestellt werden, entsprechend waren zwei Drittel aller Kinder fehlerhaft im Fahrzeug gesichert. Zwar spricht der Vergleich auf der einen Seite für gut reproduzierbare Studienergebnisse, er zeigt aber auch, dass es im Untersuchungszeitraum keine positiven Effekte aufgrund von Maßnahmen zur Reduzierung der Häufigkeit von Misuse gab. 


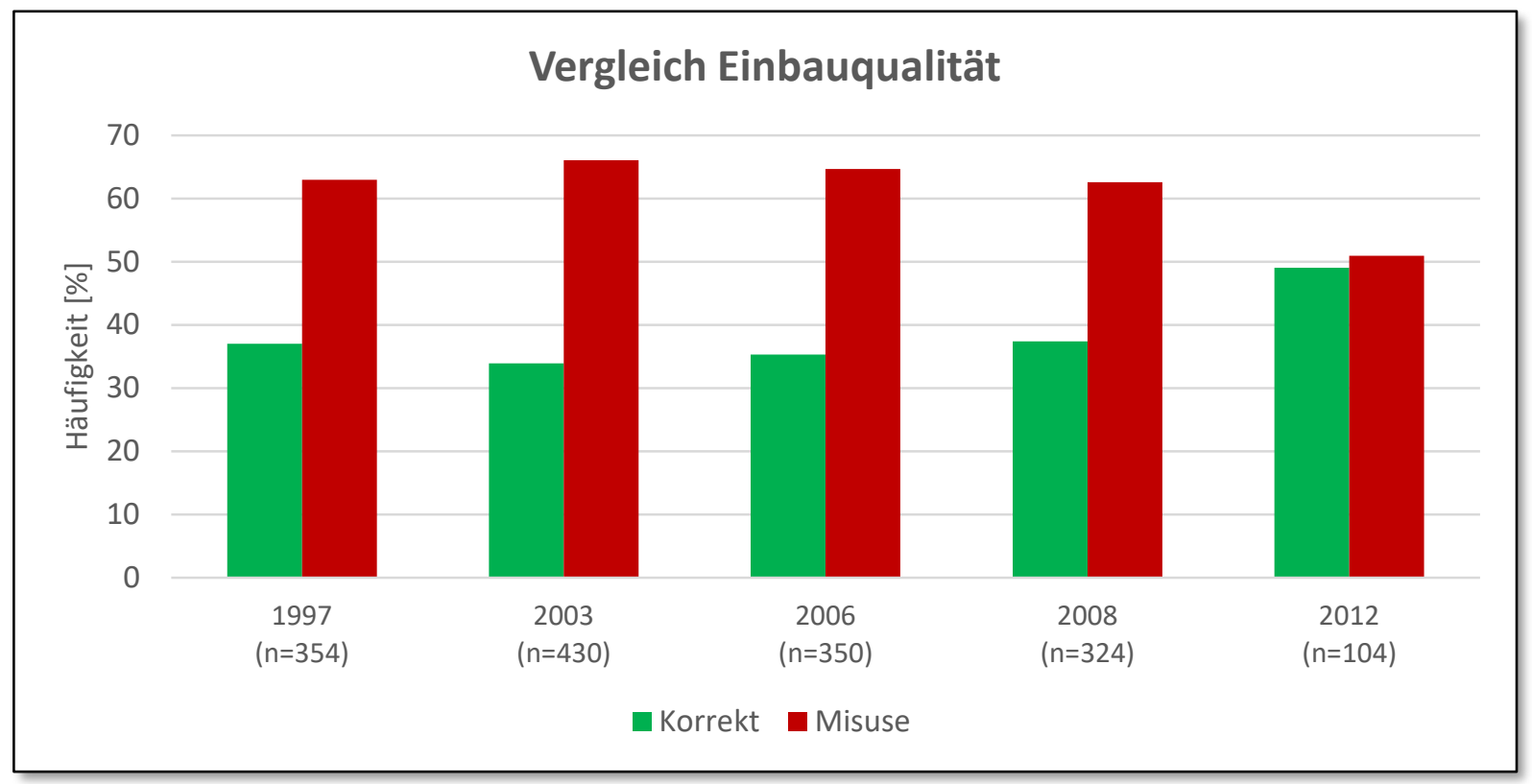

Abbildung 5: Vergleich der Misusequote verschiedener Feldstudien [Langwieder, 1997; Hummel, 2003; Fastenmeier, 2006; Hummel, 2008; Casper, 2012]

Neben der Häufigkeit von Misuse wurde in allen Feldstudien auch dessen Schwere bewertet. Ein Vergleich dieser Bewertung zeigt zumindest für die Anfangsphase eine positive Tendenz (Abbildung 6). Der Anteil der schweren Fehlbenutzung ist im Vergleich der Erhebungen 1997 und 2003 deutlich gesunken, von da an blieb die Verteilung allerdings auch konstant. Die Daten, die im Rahmen des EU-Forschungsprojekts CASPER erhoben wurden zeigen sogar, dass die Schwere des Misuse zugenommen hat. Hier ist allerdings einschränkend zu bemerken, dass diese Studie nicht nur in Deutschland, sondern auch in Frankreich und Italien durchgeführt wurde. Da Untersuchungen im Bereich der Kindersicherheit aber auch zur Fahrzeugsicherheit im Allgemeinen immer wieder zeigen, dass es zu diesem Thema erhebliche regionale Unterschiede gibt, ist davon auszugehen, dass dieser Faktor auch auf die Ergebnisse der CAPSER-Studie einen Einfluss hat.

Der positive Verlauf der Entwicklung der Misuseschwere im Vergleich der ersten vier Felderhebungen dürfte im Wesentlichen auf die Überarbeitung der Zulassungsvorschrift ECE-R 44 zurückzuführen sein. Im Jahr 1996 wurde die Regelung 44.02 durch die Regelung 44.03 abgelöst. In dieser neuen Regelung wurden zahlreiche Vorschriften mit dem Ziel überarbeitet, Fehlbenutzungen zu reduzieren. Während in der erstgenannten Studie, die 1995 erhoben wurde, ausschließlich Kindersitze untersucht wurden, die nach ECE-R 44.02 zugelassen wurden, dürfte deren Anteil in der Nachfolgestudie schon sehr gering gewesen sein. Ein wesentlicher Unterschied zwischen den Studien zeigt sich im Vergleich der Babyschalen. Während in der ersten Studie ein hoher Misuseanteil bei Gruppe 0-Sitzen 
festzustellen war, ging dieser bei den Folgestudien für 0+-Sitze deutlich zurück. Diese Sitze der Gruppe 0+ waren aber bereits nach der ECE-R 44.03 zugelassen, was bedeutet, dass sie entsprechend der neuformulierten Zulassungsverordnung über farbige Gurtmarkierungspunkte verfügen müssen. Das kann als Ursache für die Reduzierung von Fehlern bei der Einbaurichtung und bei der Führung des Fahrzeuggurts angenommen werden [vgl. Johannsen, 2007].

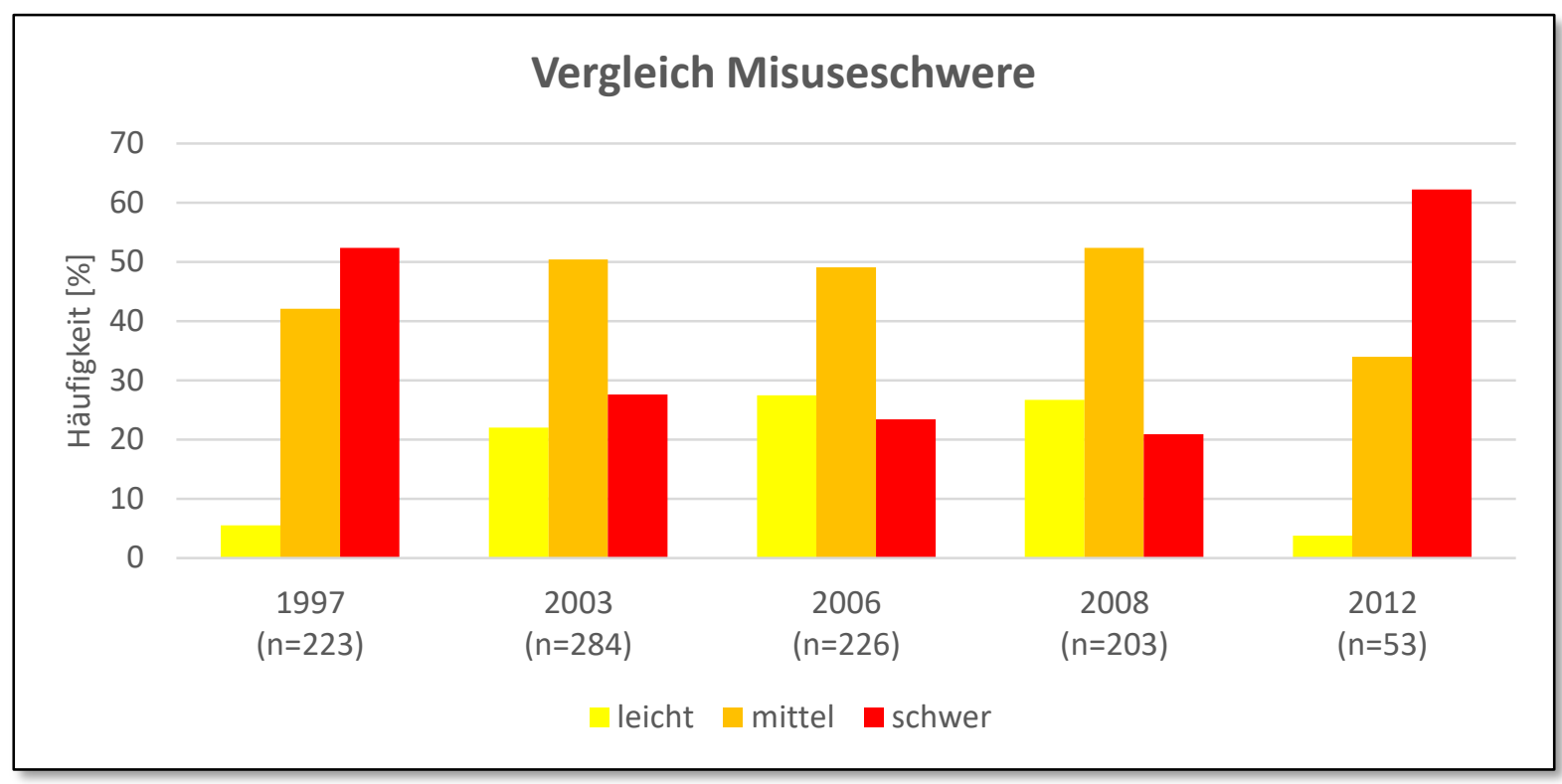

Abbildung 6: Vergleich der Verteilung der Misuseschwere [Langwieder, 1997; Hummel, 2003; Fastenmeier, 2006; Hummel, 2008; Casper, 2012]

Weiterhin wurde in den Feldstudien detailliert untersucht, bei welcher Kindersitzgruppe welcher Fehler mit welcher Häufigkeit auftritt und es wurden mögliche Zusammenhänge zwischen der Sicherungssituation und den Beförderungsumständen untersucht. Zusätzlich wurde in der Regel ein Kurzinterview mit dem Fahrer des Fahrzeugs durchgeführt, in dem Fragen zur Sicherung des Kindes und zur Einschätzung der Sicherung gestellt wurden.

Insgesamt zeigten die Feldstudien, dass die fehlerhafte Nutzung von Kindersitzen in den allermeisten Fällen durch ein Fehlverhalten seitens der Nutzer verursacht wird. Das geschieht zu einem großen Teil aus Nachlässigkeit, in seltenen Fällen wird eine Beschränkung der Sicherheit zugunsten einer vermeintlichen Komfortverbesserung allerdings bewusst in Kauf genommen. In wenigen Fällen ist die Fehlbenutzung auf rein technische Ursachen zurückzuführen. Beispiele dafür sind ein zu kurzer Fahrzeuggurt oder grundlegende Passungsprobleme zwischen Kindersitz und Fahrzeug. 
Nicht zuletzt haben die Studien gezeigt, dass die grundsätzliche Bereitschaft der Eltern ihr Kind richtig zu sichern, durchaus vorhanden ist. Allerdings führen häufig Unwissenheit oder ein falsches Verständnis davon, wie gesichert werden sollte, zu den Fehlbenutzungen. Offensichtlich besteht weiterhin ein großer Bedarf, den richtigen Gebrauch von Kindersitzen zu vereinfachen. Gleichzeitig sollte es Ziel der Hersteller von Sicherungssystemen sein, dafür Sorge zu tragen, dass auch häufig festgestellte Fehlbenutzungsarten nicht automatisch zu einem erheblichen Verlust des Schutzpotentials von Kindersitzen führen. Beispielsweise sei hier der vertauschte Gurt bei Babyschalen genannt, der regelmäßig in etwa der Hälfte der untersuchten Babyschalen festzustellen ist und im Ergebnis zu einer nicht im Fahrzeug sicher befestigten Babyschale führt.

\subsection{Bisherige Maßnahmen zur Vermeidung von Misuse}

Zur Vermeidung der Fehlbenutzung von Kindersitzen hat es in den letzten Jahren zahlreiche Aktivitäten gegeben. Dabei wurde der gesetzliche Rahmen zur Zulassung von Kindersitzen verändert. Seitens einiger Verbraucherschutzorganisationen wurden Tests mit erheblich höheren Anforderungen als die der gesetzlichen Zulassungstests eingeführt, es wurden Kampagnen und Schulungsmaßnahmen zur richtigen Benutzung von Kindersitzen ins Leben gerufen und nicht zuletzt haben viele Hersteller von Kindersitzen technische Neuerungen auf den Markt gebracht, die den Misuse verhindern sollen.

Im Folgenden werden wesentliche Maßnahmen und Konzepte vorgestellt und kurz erörtert, wobei diese Darstellung keinen Anspruch auf Vollständigkeit erhebt.

\subsubsection{Gesetzliche Maßnahmen}

Mit der Einführung der überarbeiteten ECE-Regelung zur Zulassung von Kindersitzen im Jahr 1995 (ECE-R 44.03) wurden einzelne Problemfelder mit großem Misusepotential adressiert. So ist es seitdem verpflichtend, dass der Verlauf des Fahrzeuggurts am Kindersitz einheitlich farblich markiert wird. Für rückwärtsgerichtete Kindersitze sind entsprechende Stellen blau zu markieren, für vorwärtsgerichtete KSS rot. Der häufig festgestellten Problematik der falschen Gurtführung bzw. der falschen Einbaurichtung des Kindersitzes sollte damit begegnet werden.

Der zu frühe Wechsel der Kinder von der Babyschale in einen vorwärtsgerichteten Sitz wurde mit der Einführung einer neuen Kindersitzgruppe adressiert. Die Gruppe 0+ gilt seither für ein Gewicht des Kindes bis $13 \mathrm{~kg}$ und gilt ergänzend zur Gruppe 0 (bis $10 \mathrm{~kg}$ ). Damit soll erreicht werden, dass Kinder länger in rückwärtsgerichteten Sitzen befördert 
werden. Als Ergebnis dieser Maßnahme ergibt sich ein großer Überschneidungsbereich mit der Gruppe 1 ( 9 bis $18 \mathrm{~kg}$ ), da diese nicht entsprechend angepasst wurde.

Das Problem eines schlecht positionierten Gurtschlosses im Gurtsystem des Kindersitzes wurde mit der Einführung eines fünften Gurts weitestgehend gelöst. Durch den Schrittgurt befindet sich das Gurtschloss an einem definierten Ort und kann nicht in den Abdominalbereich des Kindes hochrutschen. Die vorher üblichen 4-Punkt-Gurte sind seitdem nicht mehr zulassungsfähig, gleiches gilt für die sogenannte Gurtmanschette (siehe Abbildung 7).

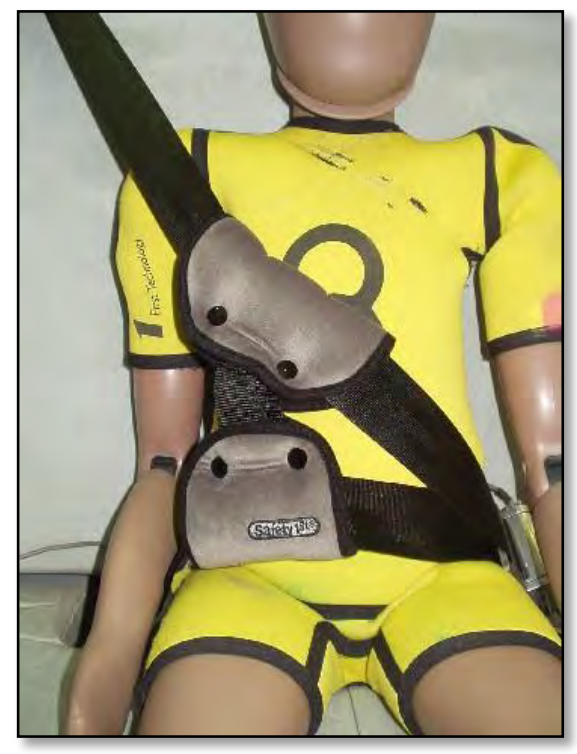

Abbildung 7: Dummy mit Gurtmanschette [Johannsen, 2006]

In einer weiteren Überarbeitung der ECE-R 44.03 wurde das ISOFIX-System als standardisierte, feste mechanische Verbindung zwischen Kindersitz und Fahrzeug ergänzt. Im Zusammenhang mit Bemühungen, die Fehlbenutzungsrate von Kindersitzen im Fahrzeug zu reduzieren, nimmt dieses System sowohl hinsichtlich seines Potentials als auch hinsichtlich seines Beispiels für die Komplexität des gesamten Themenbereichs eine herausragende Rolle ein. Das ISOFIX-System wurde entwickelt, um Fehler im Bereich der Schnittstelle zwischen Kindersitz und Fahrzeug zu vermeiden. Dabei wird der Kindersitz nicht durch den Fahrzeuggurt, sondern mittels einer festen, mechanischen Verankerung mit dem Fahrzeug verbunden. Am Kindersitz selbst sind zwei Haken befestigt, die in entsprechende Verankerungen am Fahrzeug einrasten (Abbildung 8). 


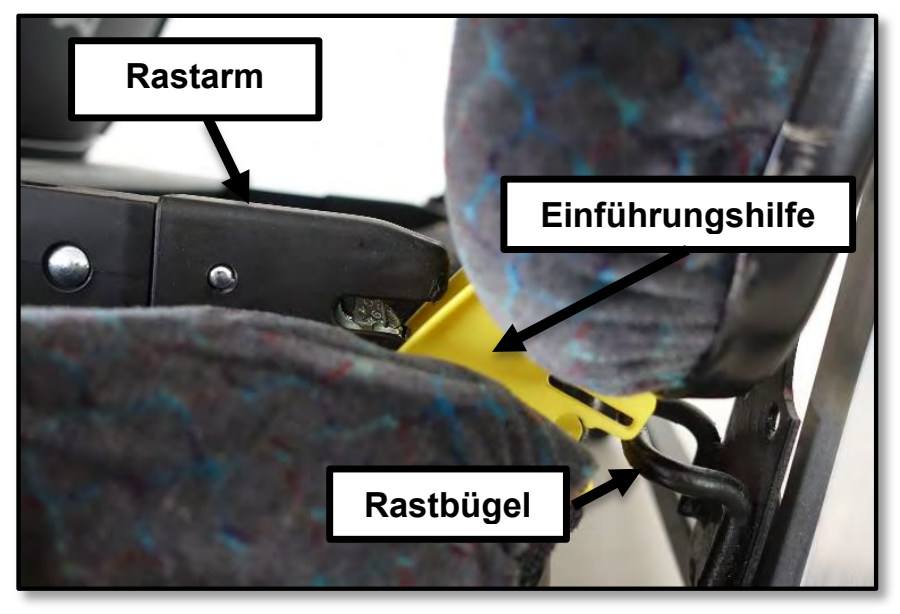

Abbildung 8: Prinzipskizze des ISOFIX-Systems

Mit Hilfe dieses Systems können alle maßgeblichen Fehler, die bei der Installation eines Kindersitzes im Fahrzeug auftreten können, vermieden werden.

Aufgrund der Konstruktionsweise des ISOFIX-Systems ergibt sich prinzipbedingt, dass der Kindersitz im Fall einer Verzögerung um die Achse durch die beiden Befestigungspunkte rotieren kann, was eine unerwünschte Vorverlagerung des Insassen zur Folge hat.

Um dieser Problematik zu begegnen, war es notwendig eine Antirotationseinrichtung zu entwickeln. Dabei haben sich zwei Lösungen durchgesetzt: Der Top Tether und der Stützfuß. Beim Top Tether wird der Kindersitz mittels eines Gurts, der an der Oberseite des Sitzes befestigt ist, nach hinten abgespannt (Abbildung 9). Beim Stützfuß stützt sich der Kindersitz direkt am Fahrzeugboden ab (Abbildung 10). Beide Systeme verhindern die Rotation wirkungsvoll, können aber gleichzeitig Ausgangspunkt weiterer Fehlbenutzungen sein.

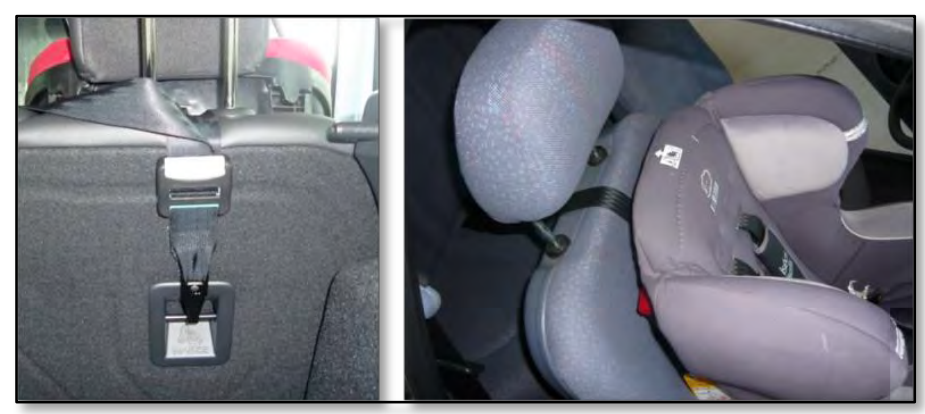

Abbildung 9: Gruppe 1 Sitz mit Top Tether Links: Gurt ist an vorgesehenem Punkt eingehängt. Ein Indikator zeigt an, dass der Gurt ausreichend gespannt ist. Rechts: Top Tether wird von der Rückseite des Kindersitzes über die Rückenlehne geführt 


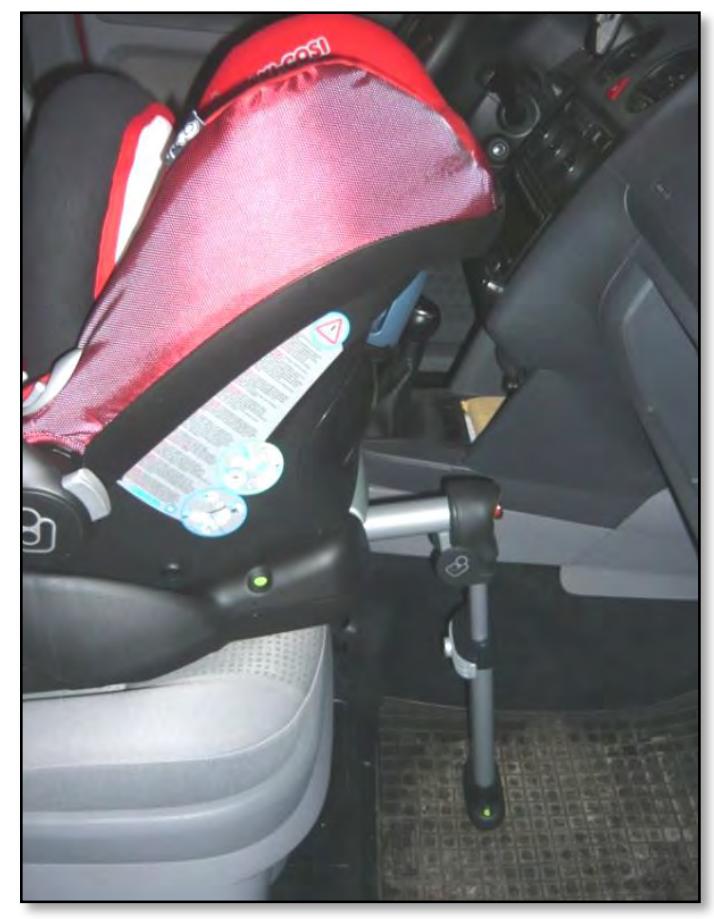

Abbildung 10: Stützfuß an der Vorderseite einer Basis, auf der eine Babyschale montiert ist. Indikatoren zeigen durch grüne Farbgebung an, dass die Babyschale richtig eingerastet ist und dass der Stützfuß ausreichend Bodenkontakt hat.

Um einen mit einem Top Tether ausgestatteten ISOFIX-Sitz verwenden zu können, ist es erforderlich, dass ein entsprechender Verankerungspunkt dafür im Fahrzeug vorhanden ist. Ein solcher wurde bisher nicht serienmäßig an allen mit ISOFIX-Haken ausgestatteten Plätzen verbaut. Entsprechend kann es dazu kommen, dass ein solcher Sitz ohne den oberen Spanngurt verwendet wird, da das Fahrzeug nicht über die entsprechende Aufnahme verfügt.

ISOFIX-Sitze mit einem Stützfuß können prinzipiell in allen Fahrzeugen verwendet werden, Einschränkungen gibt es hierbei allerdings, wenn sich im Boden des Fahrzeugs ein Staufach befindet. In diesem Fall kann es sein, dass der Deckel des Staufachs die auftretenden Kräfte möglicherweise nicht aufnehmen kann, entsprechend untersagen die meisten Fahrzeughersteller die Verwendung eines Stützfußes in dieser Konstellation [Cehic, 2012]. Vereinzelt werden Lösungen angeboten; so bieten einzelne Hersteller über den Zubehörhandel Füllmaterial an, mit dem das Staufach ausgefüllt werden kann, wodurch der Deckel abgestützt wird. Weiterhin kann es Probleme mit der Länge des Stützfußes geben oder er kann mit anderen Fahrzeugteilen, wie beispielsweise der Mittelkonsole, in Kontakt kommen, weshalb ein Einbau nicht möglich ist. Generell sind diese Kindersitze semiuniversal zugelassen, das heißt es liegt in der Verantwortung der Kindersitzhersteller 
sicherzustellen, dass der Kindersitz in den auf der zugehörigen Liste genannten Fahrzeugmodellen installierbar ist.

Jenseits dieser beiden Einflussgrößen (Top Tether und Stützfuß) gilt für ISOFIX-Sitze generell, oder zumindest für die allermeisten Modelle, dass sie keine Universalzulassung haben. Das heißt, jeder Sitz ist für bestimmte Fahrzeugmodelle zugelassen, die auf einer zugehörigen Liste vermerkt sind. Der Käufer muss sich anhand dieser Fahrzeugliste informieren, ob er den gewählten Sitz in seinem Fahrzeug überhaupt verwenden darf.

Neben der beschriebenen gesetzlichen Regelung ECE-R 44 wurde parallel die ECE-R 129 etabliert. Diese Regelung beschreibt ebenfalls Rahmenbedingungen, nach denen ein Kindersitz zugelassen wird. Langfristig soll sie die R44 ablösen. Die neue gesetzliche Regelung soll in zwei Phasen in Kraft treten, aktuell ist nur die Phase 1 verabschiedet worden. Gemäß dieser Regelung können nur KSS zugelassen werden, die mit ISOFIX ausgestattet sind. Diese Sitze sind mit dem Namen i-Size gekennzeichnet. Die ISOFIXAnbindung ist dabei standardisiert, d.h., sie ist mit neuen Fahrzeugen passungsfähig, deren ISOFIX-Verankerung ebenfalls als i-Size kompatibel gekennzeichnet ist. Aktuell gibt es jedoch nur wenige KSS, die nach dieser Norm zugelassen wurden.

Im Gegensatz zu den früheren Regelungen gibt es in der ECE-R 129 keine feste Einteilung der Kindersitze nach Gewichtsklassen mehr. Vielmehr werden die Kindersitze gemäß der Größe der Kinder zugeordnet und es obliegt dem Kindersitzhersteller, dafür eine Festlegung vorzunehmen. Eingeschränkt wird lediglich, dass der Hersteller dafür Sorge zu tragen hat, dass Kinder bis zum 15. Lebensmonat rückwärtsgerichtet im Fahrzeug befördert werden müssen.

Aus Sicht der Sicherheit wurden die Anforderungen mit der neuen Gesetzgebung verschärft. Als Messpuppen werden die deutlich biofideleren Dummys der Q-Serie eingesetzt. Neben dem Frontalaufprall werden KSS auch im Seitenaufprall getestet.

\subsubsection{Maßnahmen seitens Verbraucherschutzorganisationen}

Zahlreiche Verbraucherschutzorganisationen bieten Informationsbroschüren über die richtige Nutzung von Kindersitzen an. Dazu zählen der ADAC, der Autoclub Europa (ACE), der Deutsche Verkehrssicherheitsrat (DVR), die deutsche Verkehrswacht (DVW), der Gesamtverband der Deutschen Versicherungswirtschaft (GDV), einzelne Landesverkehrswachten und weitere Organisationen. Gutes Informationsmaterial ist aber auch seitens staatlicher Einrichtungen, wie beispielsweise der Bundesanstalt für Straßenwesen erhältlich. Diese Broschüren beinhalten grundlegende Informationen zum richtigen Umgang mit Kindersitzen, weisen auf häufige Fehler und deren Folgen hin und 
geben Tipps zum Erwerb von Kindersitzen. Je nach Ausführlichkeit und Detailtiefe dieser Informationen sind sie für Eltern eine nützliche Ergänzung zur Bedienungsanleitung des Kindersitzes.

Einige Organisationen haben es sich zur Aufgabe gemacht, das Schutzpotential von Kindersitzen zu testen. Dabei werden Anforderungen gestellt, die über die gesetzlichen Vorgaben hinausgehen.

Sehr aktiv auf diesem Gebiet sind in enger Zusammenarbeit der ADAC und die Stiftung Warentest. Im halbjährlichen Rhythmus werden Kindersitze getestet, die neu auf dem Markt erschienen sind. Abweichend vom gesetzlichen Zulassungstest wird eine reale Fahrzeugverzögerung simuliert, der Kindersitz selbst wird dabei in einer Fahrzeugumgebung getestet und nicht auf einer normierten Sitzbank, neben dem Frontalaufprall wird auch der Seitenaufprall geprüft. Als Prüfkörper werden anstelle der P-Dummys die weiter entwickelten Q-Dummys verwendet. Der Sitz wird anhand der gemessenen Belastungswerte am Dummy bewertet. Somit ergeben sich für den Verbraucher verschiedene Vorteile: zum einen erfährt er je nach Testprädikat (sehr gut, gut, befriedigend, mangelhaft, ...), wie gut ein bestimmtes Modell ist, gleichzeitig ergibt sich mit dieser Abstufung eine Vergleichbarkeit der Kindersitze untereinander. Neben der rein technischen Überprüfung werden bei diesem Testverfahren auch die Handhabbarkeit und die Anfälligkeit für Fehler getestet, was ebenfalls ein grundsätzlicher Unterschied im Vergleich zum Zulassungsverfahren ist. Das Ergebnis fließt ebenfalls in die Gesamtbewertung mit ein.

Neben den Kindersitztests durch den ADAC gibt es noch andere Einrichtungen, die in unregelmäßigen Abständen Testserien durchführen. Beispielsweise genannt seien hier Automobilzeitschriften wie Auto, Motor und Sport oder Autobild.

Ebenfalls sehr präsent in der öffentlichen Wahrnehmung sind die Fahrzeugtests, die gemäß den Vorgaben von EuroNCAP durchgeführt werden. Die Ergebnisse werden regelmäßig in den Medien publiziert und von den entsprechenden Fahrzeugherstellern werbewirksam eingesetzt. Im Rahmen dieser Fahrzeugtests wird auch der Schutz von Kindern überprüft, womit also auch die Schutzwirkung von Kindersitzen in der entsprechenden Kombination mit dem Pkw getestet wird. Die Auswahl des Kindersitzes obliegt dabei dem Fahrzeughersteller. Getestet werden ein Frontal- und ein Seitenaufprall, wobei mittlerweile ein 6- und ein 10jähriger Dummy aus der Q-Serie eingesetzt werden. Bewertet werden die Kinematik des Dummys und Belastungen, die sich aus der Auswertung von Beschleunigungswerten ergeben. Weiterhin wird im Bewertungsverfahren die Ausstattung des Fahrzeugs hinsichtlich Kindersicherheit bewertet. Dabei liegen das ISOFIX-System und die Möglichkeit der Airbagabschaltung im Fokus. 
Darüber hinaus findet eine Einbauprüfung im Fahrzeug statt. Kindersitze, die in unabhängigen Tests gute Ergebnisse erzielt haben, werden im Fahrzeug installiert. Länge des Gurts, Position des Gurtschlosses, Nutzungsmöglichkeit der ISOFIX-Anbindung sowie die Stabilität des KSS im Fahrzeug werden für alle Einbaupositionen untersucht.

Die grundsätzliche Struktur des Kindersicherheitstests bei EuroNCAP ist in Anhang 6.4 dargestellt.

\subsubsection{Technische Maßnahmen}

Mit dem Ziel, die Fehlbenutzungsquote zu reduzieren, gibt es auch seitens der Kindersitzhersteller zahlreiche Lösungsansätze für bestimmte Probleme. Dabei wird zum Teil mit mechanischen Indikatoren gearbeitet, die anzeigen, ob eine entsprechende Einrastvorrichtung ordnungsgemäß funktioniert hat oder ob ein Gurt genügend gespannt wurde. In beiden Fällen wird dem Nutzer durch einen einfachen Mechanismus angezeigt, ob die entsprechende Sicherungsvorrichtung korrekt genutzt wurde. Als Beispiele seien hier der "Visual Harness Tension Indicator" der Firma Britax Römer (Abbildung 11) sowie der Maxi Cosy Cabrio mit seiner Basis „Easybase“ (Abbildung 12) gezeigt.

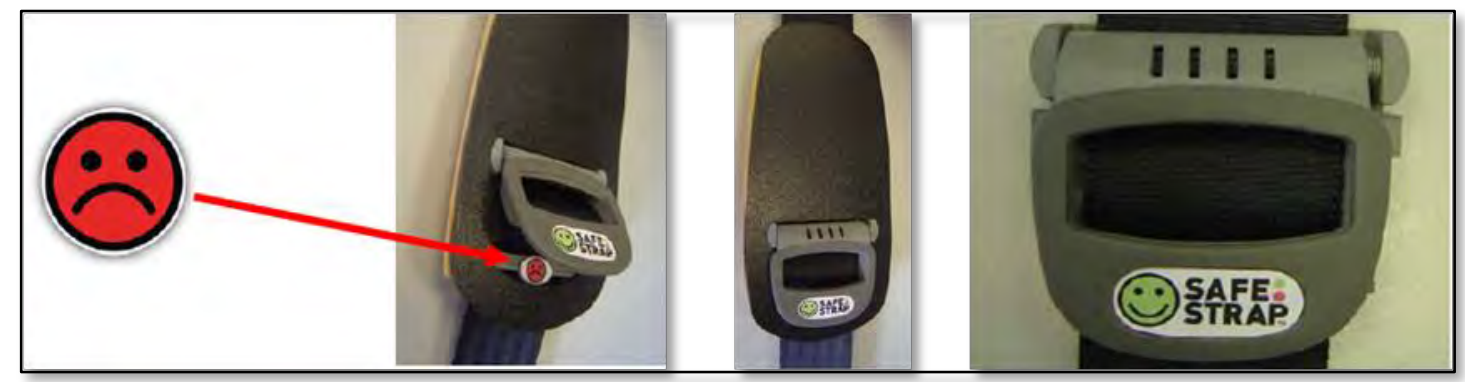

Abbildung 11: Anzeige zur Gurtspannkraft Links: Gurtspannkraft ist ungenügend, ein rotes Smiley ist zu sehen. Mitte, rechts: Gurtspannkraft ist ausreichend, dem Nutzer wird ein grünes Smiley angezeigt. [Bendjellal, 2006] 

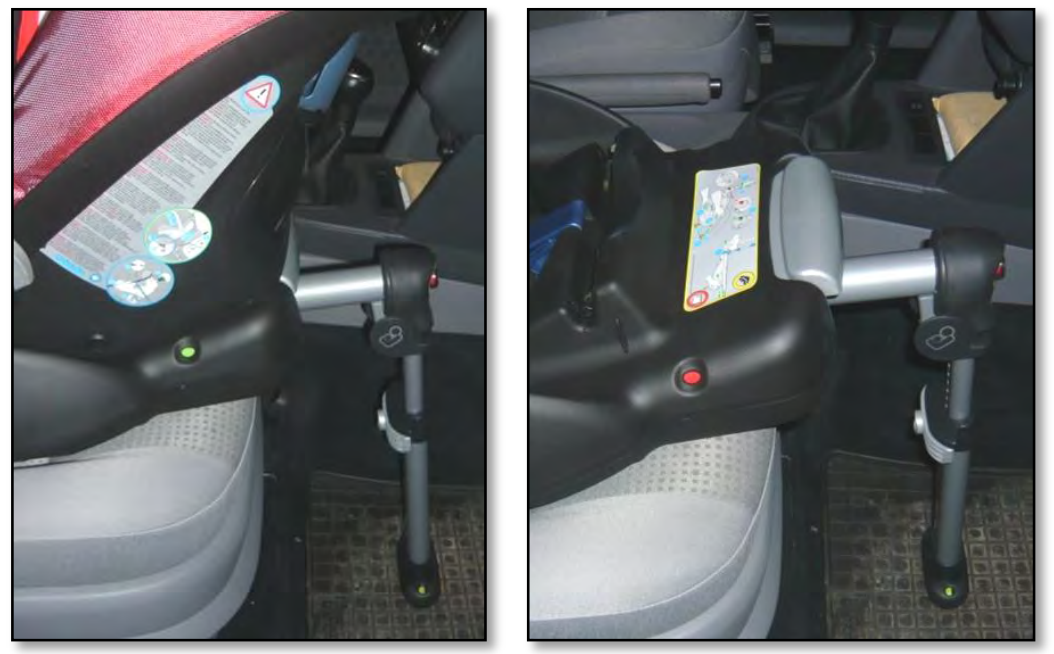

Abbildung 12: Anzeige zur richtigen Sicherung einer Babyschale. Links: Die Babyschale ist richtig auf der Sitzbasis eingerastet, der Indikator zeigt einen grünen Punkt. Rechts: Die Sitzschale ist nicht richtig eingerastet, der Indikator ist rot.

Ähnliche Systeme existieren zur Anzeige der richtigen Nutzung der ISOFIX-Verankerung, der richtigen Einstellung eines Stützfußes, zur Anzeige der hinreichenden Spannkraft beim Top Tether usw.

In vergleichbarer Weise funktionieren akustische Warnsignale. Diese geben einen Ton ab, wenn bestimmte Einstellungen nicht wie vorgesehen vorgenommen wurden. Solche Systeme können ebenfalls zur Überwachung der Gurtkraft und vor allem zur Überwachung des Gurtschlosses eingesetzt werden, aber auch andere mechanische Verbindungen und Einstellungen lassen sich überprüfen (ISOFIX, Stützfuß, ...). Akustische Warnungen haben den Vorteil, dass sie den Nutzer auch während der Fahrt zum Beispiel vor einem geöffneten Gurtschloss warnen können. Damit wird nicht nur die Einbausituation überwacht, sondern auch die Funktionsweise bestimmter Komponenten während der Fahrt.

Ein Prototyp für einen Kindersitz der Gruppe 1, der alle wesentlichen auftretenden Misusearten detektiert und anzeigt, wurde in einem studentischen Forschungsprojekt an der TU Berlin entwickelt (Abbildung 13). Dabei wurde von Beginn an die Idee verfolgt, dass jeder denkbare Fehler erkannt und dem Nutzer gleichzeitig ein Lösungsvorschlag angezeigt werden soll. Als Schnittstelle zum Anwender wird ein Monitor mit einem zweizeiligen Textfeld verwendet. Die Benutzung erfolgt dabei in zwei Schritten: Zunächst wird der Sitz mittels des Fahrzeuggurts im Pkw befestigt. Dabei werden der richtige Gurtpfad, die hinreichende Gurtspannung sowie die richtige Positionierung des Sitzes im Fahrzeug überwacht. Wird ein Fehler erkannt, erhält der Nutzer über das Display einen Lösungsvorschlag. Bei der Sicherung des Kindes im KSS werden sowohl die Gurtkraft, die richtige Höheneinstellung des Schultergurts und das Gurtschloss überwacht. Wird das 
Gurtschloss während der Fahrt geöffnet, ertönt eine Warnung. Wie in zahlreichen Versuchen mit erfahrenen und unerfahrenen Kindersitznutzern nachgewiesen werden konnte, unterbindet der Sitz verlässlich alle Fehlbedienungsarten [Müller, 2009].

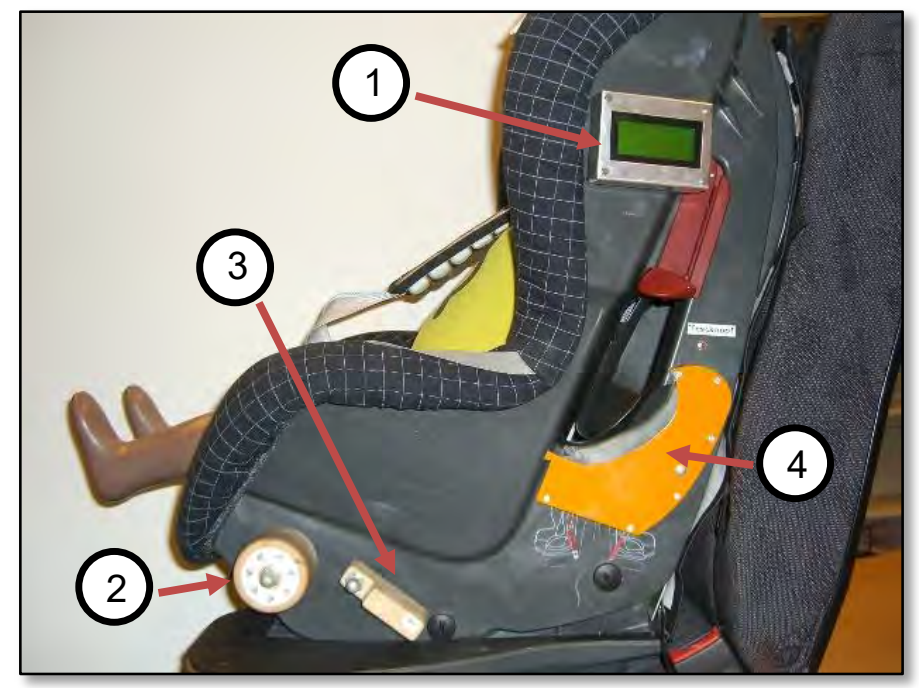

Abbildung 13: Prototyp eines KSS zur Vermeidung von Misuse mit Display (1) als Schnittstelle zum Nutzer, Rutschkupplung (2), zentraler Lösemechanismus (3), Sensor zur Ermittlung der Gurtkraft (4)

Auch seitens der Fahrzeughersteller wurden Systeme entwickelt, die Misuse erkennen und verhindern sollen. Ein Beispiel dafür ist "Child Seat Presence and Orientation Detection“ (CPOD). Dabei handelt es sich um ein System, welches die Art und die Einbaurichtung eines Kindersitzes auf dem Beifahrersitz erkennt. Befindet sich dort ein rückwärtsgerichteter Kindersitz, wird automatisch der Beifahrerairbag deaktiviert [Brämig, 2006]. Damit dieses System ordnungsgemäß funktioniert, müssen allerdings der Kindersitz mit einem Transponder und das Fahrzeug mit einem zugehörigen Empfänger ausgestattet sein. Daraus ergeben sich aber auch neue potentielle Ursachen für die Fehlbenutzung.

Zunehmend nachgefragt sind Systeme, deren Funktion es ist, das Sichern des Kindes zu erleichtern. Dazu zählen beispielsweise Kindersitze, die sich zum Einsetzen des Kindes um $90^{\circ}$ zur Fahrtrichtung drehen lassen (s. Abbildung 14). 


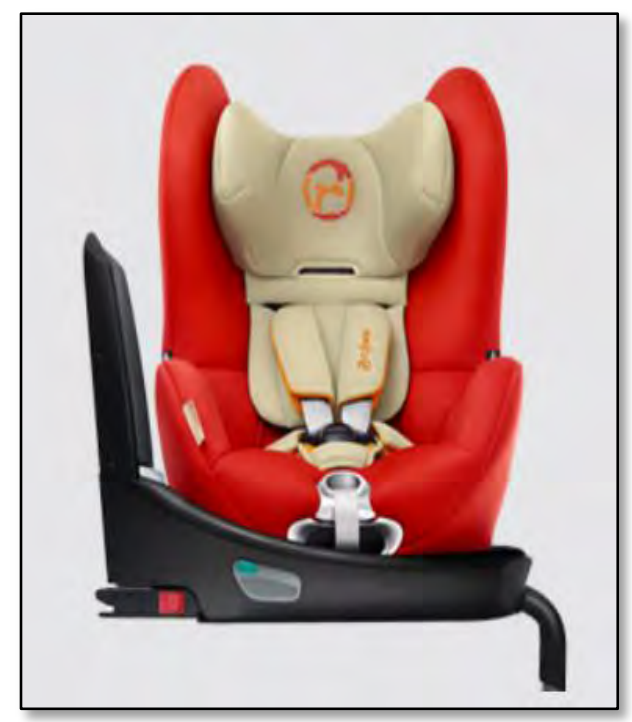

Abbildung 14: Drehbares KSS der Klasse 1 [Cybex, 2017]

Im dargestellten Beispiel besteht überdies die Möglichkeit, den Kindersitz sowohl vorwärtsals auch rückwärtsgerichtet im Fahrzeug zu verwenden. Das ermöglicht einen fließenden Übergang zwischen beiden Sicherungsvarianten. 


\section{Felderhebungen zu Misuse}

Um die Häufigkeit und die Schwere von Misuse in der Realität zu überprüfen und zu bewerten, wurde im Rahmen des hier vorgestellten Forschungsprojekts eine Misusestudie durchgeführt. Dabei wurden reale Sicherungssituationen von Kindern im Pkw analysiert. Ergänzend wurde mit dem Fahrer des jeweiligen Pkw ein Kurzinterview durchgeführt. Je nach Bereitschaft der interviewten Person wurde zusätzlich ein vertiefendes Telefoninterview durchgeführt.

Im folgenden Kapitel wird der Aufbau und Struktur der Fragebögen und die Durchführung der Feldbefragung beschrieben. Wesentlich bei der Konzeptionierung war das Ziel eine möglichst große Vergleichbarkeit $z u$ den in der Vergangenheit durchgeführten Misusestudien zu ermöglichen.

\subsection{Erhebungsinstrumente}

Um über möglichst alle interessierenden Aspekte von Misuse Erkenntnisse zu erhalten, mussten sowohl Daten über die Häufigkeit, Schwere etc. von Misuse als auch über die zugrundeliegenden Kenntnisse, Einstellungen und Fertigkeiten der Nutzer erhoben werden. Deshalb wurde für die Feldstudie zu Misuse ein zweistufiges Beobachtungs- und Befragungskonzept erarbeitet. Zur Erfassung von Misuse-Formen, den innen zugrundliegenden Handlungsmotiven sowie relevanter Kontextbedingungen im Feld wurden zwei Erhebungsinstrumente entwickelt. Beide Erhebungsinstrumente - Beobachtungsbogen und Fragebogen für die Nachbefragung - basieren auf den im Rahmen der Systematisierung von Misuse-Ursachen entwickelten Hypothesen aus den Arbeiten der Vorgängerstudien.

\section{Beobachtungsbogen}

Im Zentrum der Beobachtungen an Kontaktplätzen (z.B. Supermarkt, Freizeiteinrichtungen, Kindergarten, Schule) stand die Erfassung von Misuse-Formen. Ungesicherte Kinder und mit Erwachsenengurten gesicherte Kinder wurden mit diesem Instrument ebenfalls erfasst; weitere Daten zu dieser Teilgruppe wurden jedoch wegen der Fokussierung auf die MisuseFormen nicht erhoben. Der Beobachtungsbogen (vgl. Anhang 6.1) umfasste neben der Dokumentation der Witterungsbedingungen und des Kontaktplatzes insgesamt vier Teile und zwar:

- Angaben zu Fahrzeug und Insassen,

- Angaben zur interviewten Person,

- Angaben zu Kind(ern), KSS und Misuse, 
- Fragen zum festgestellten Misuse.

\section{Misuse-Formen}

Als Misuse bzw. fehlerhafte Nutzung von KSS wird hier jede Abweichung vom intendierten Gebrauch eines KSS, die die Schutzwirkung des KSS reduzieren könnte, verstanden. Für die Einstufung des erfassten Misuse wurde die schon in den Vorgängerprojekten jeweils (weiter)entwickelte Checkliste verwendet und in ihrer Struktur aber entscheidend vereinfacht. Jetzt orientiert sich die Checkliste in ihrer Gliederung an den KSS-Systemarten, denen jeweils die dort auftretenden Misuse-Formen zugeordnet sind. Eine Einschätzung der Misuse-Schwere (leicht, mittel, schwer) erfolgt a priori lediglich für ausgewählte Fehlbenutzungen, alle anderen werden nachträglich eingestuft.

Für die hier vorgestellte Studie wurde das Codebook erneut überarbeitet und an aktuelle Entwicklungen angepasst. Dazu gehören neben neuen gesetzlichen Rahmenbedingungen auch die Entwicklung neuer Sicherungssysteme. Das vollständige Codebook ist im Anhang (6.2) dargestellt.

Das Codebook besteht aus drei Teilen. Im ersten Abschnitt sind die möglichen Fehlerarten für Klasse 0/0+-Sitze aufgeführt, im zweiten Abschnitt die Fehlerarten der Klasse 1-Sitze und im dritten Abschnitt entsprechend die Misusevarianten für Klasse 2/3-Sitze. Das gilt auch für die jeweilige Entsprechung der Sitzmodelle, die nach der ECE-R 129 zugelassen sind.

Innerhalb der einzelnen Abschnitte sind die Fehlerarten nach „allgemeinen Fehlern“, „Fehlern, die die Sicherung im Pkw betreffen“, „Fehlern, die die Sicherung des Kindes im KSS betreffen“, „Fehlern im Zusammenhang mit ISOFIX“ und „sonstigen Fehlern“ unterteilt. Jeder dieser Untergruppen sind entsprechend bestimmte Fehler zugeordnet, die wiederum mit einem dreistelligen Code bezeichnet sind. Demnach lässt sich jeder Fehler eindeutig codieren und für statistische Auswertungen sinnvoll erfassen. Weiterhin ist jedem Fehler ein Schweregrad zugeordnet $(\mathrm{l}=$ leicht; $\mathrm{m}=$ mittel; $\mathrm{s}=$ schwer; $\mathrm{km}=$ kein Misuse, $\mathrm{nur}$ Auffälligkeiten).

\section{Fragebogen}

Der Erfassung von Kontextbedingungen, Handlungsmotiven und handlungsrelevanten Wissenselementen diente die Nachbefragung bei festgestelltem Misuse für alle im Fahrzeug vorgefundenen Kinder. Der Fragebogen für die Nachbefragung umfasste ebenfalls vier Teile und zwar:

Fragen zum Erwerb des Kindersitzes, 
- Fragen zum Einbau des Kindersitzes (gilt in der Regel für KSS, bei denen Einbau und Sicherung zwei getrennte Vorgänge sind),

- Fragen zum Sichern im Kindersitz,

- allgemeine Fragen zum Sichern im Kindersitz (handlungsrelevante Wissenselemente).

Die Beobachtungs- und Befragungsinstrumente wurden im Vorfeld der Felderhebungen Pretests unterzogen, um sie hinsichtlich der Kriterien Konsistenz, Verständlichkeit und Akzeptanz zu überprüfen. Die Ergebnisse der Pretests resultierten in einer Kürzung des Erhebungsinstruments für die Nachbefragung sowie in Modifikationen einzelner Items in beiden Erhebungsinstrumenten. Schließlich dienten sie zu einer ausführlichen Schulung der eingesetzten Interviewer und Beobachter. Die Erfahrungen früherer Studien führten dazu, den Beobachtungs- und Befragungsteil zu trennen. Der mit der Befragung verbundene Zeitaufwand (ca. 30 Minuten) für die Probanden führte ehemals zu massiven Akzeptanzproblemen und stellte potentiell die Durchführung der Erhebungen insgesamt in Frage. Daher wurde den Probanden alternativ zur Vor-Ort-Befragung eine telefonische Nachbefragung vorgeschlagen, die sehr zeitnah (d.h. zwei bis drei Tage später) erfolgen sollte.

\subsection{Durchführung der Felderhebungen}

Die Erhebungen wurden von insgesamt fünf geschulten Beobachtern/Interviewern durchgeführt, davon zwei Beobachter/Interviewer in Berlin, zwei Beobachter/Interviewer in München sowie eine weitere Interviewerin für die Nachbefragung in München. Pro Erhebungstag kamen jeweils zwei Beobachter/Interviewer zum Einsatz. Die Schulungen für die Interviewer/Beobachter fanden im Juni 2016 statt. Anschließend wurden verschiedene Pretests durchgeführt, die an einer Reihe von Punkten Modifikationen der Erhebungsinstrumente und des Untersuchungsdesigns nach sich zogen. 


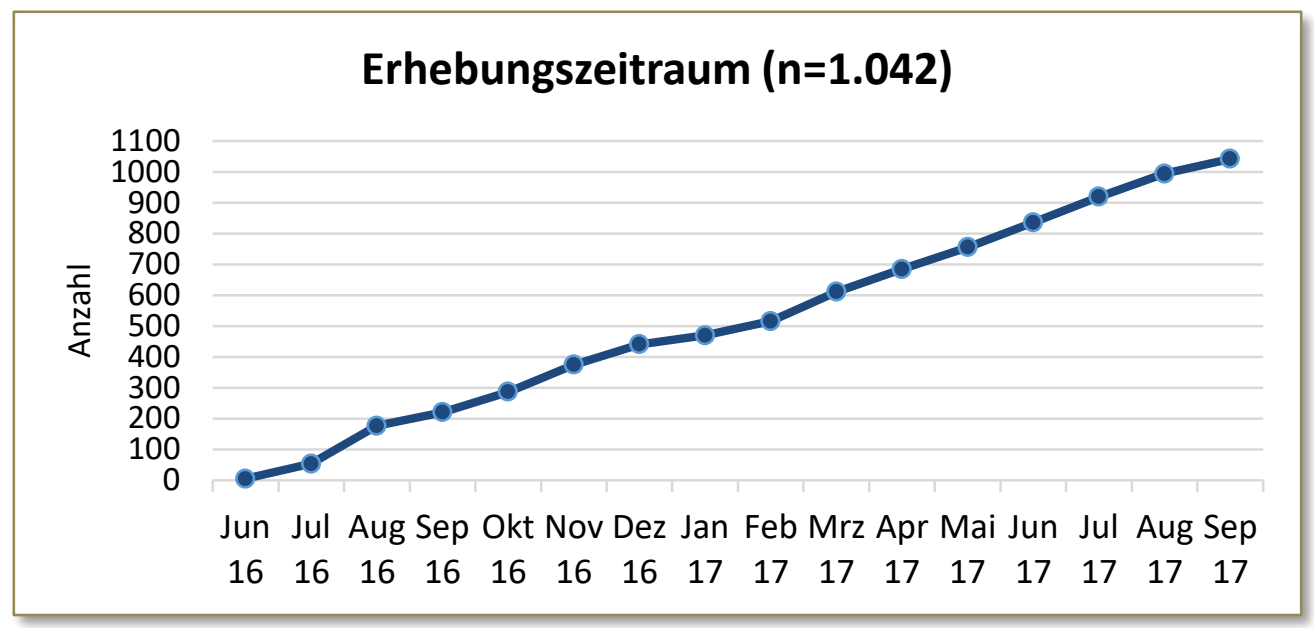

Abbildung 15: Erhebungszeitraum

Die Feldstudie wurde nach erfolgreichem Abschluss der Pretests Ende Juni 2016 begonnen und im Juni 2017 beendet. Über diesen Zeitraum wurden gleichmäßig Erhebungen durchgeführt (Abbildung 15).

Während der Studie wurden 1.076 Fälle von Kindern im Pkw erfasst, davon waren $1.042 \mathrm{im}$ Kindersitz gesichert, 34 weitere Kinder wurden ungesichert befördert. Da es das Ziel der Studie war Art und Häufigkeit von Misuse zu erheben, wurden Fälle, bei denen kein Kindersitz genutzt wurde, nur in Ausnahmefällen erhoben. Die Studie beinhaltet mehr Klasse 2/3-Sitze $(n=472)$ als Klasse 1-Sitze $(n=372)$ und als Klasse 0/0+-Sitze $(n=198)$. Diese Verteilung ergibt sich aus der Tatsache, dass 2/3-Sitze etwa ab einem Kindesalter von zweieinhalb bis drei Jahren bis zum Ende des elften Lebensjahrs genutzt werden, während die kleineren Klassen nur maximal ein Jahr bis anderthalb Jahre genutzt werden. Entsprechend sind diese Sitze deutlich häufiger anzutreffen.

Die Durchführung der Untersuchung erfolgte an ausgewählten Kontaktplätzen in den Großräumen Berlin und München. Eine Übersicht zu den Befragungsorten zeigt Abbildung 16. Eingeschlossen waren darin sowohl urbane Räume als auch Kontaktplätze in der Peripherie („Speckgürtel“) als auch weiter entfernt liegende Orte (z.B. Leipzig, Potsdam, Augsburg, Rosenheim). Mit der Wahl unterschiedlicher Kontaktplätze wurde sichergestellt, dass verschiedene Fahrtzwecke (Einkauf, Bringen/Abholen vom Kindergarten, Schule, Freizeitfahrt) im Rahmen der Erhebungen vorzufinden waren. Die Felderhebungen begannen im Juli 2016, in der Regel wochentags und tagsüber, immer wieder aber auch an Wochenenden und liefen über einen Zeitraum von ca. 1 Jahr weiter, womit eine Erhebung über alle Jahreszeiten gewährleistet ist. Damit sind auch unterschiedliche Wetterbedingungen berücksichtigt. Ausgesprochene Schlechtwettertage und Tage großer 
Kälte kamen allerdings nicht in Betracht, da dies für Interviewer und Probanden nicht zumutbar gewesen wäre.

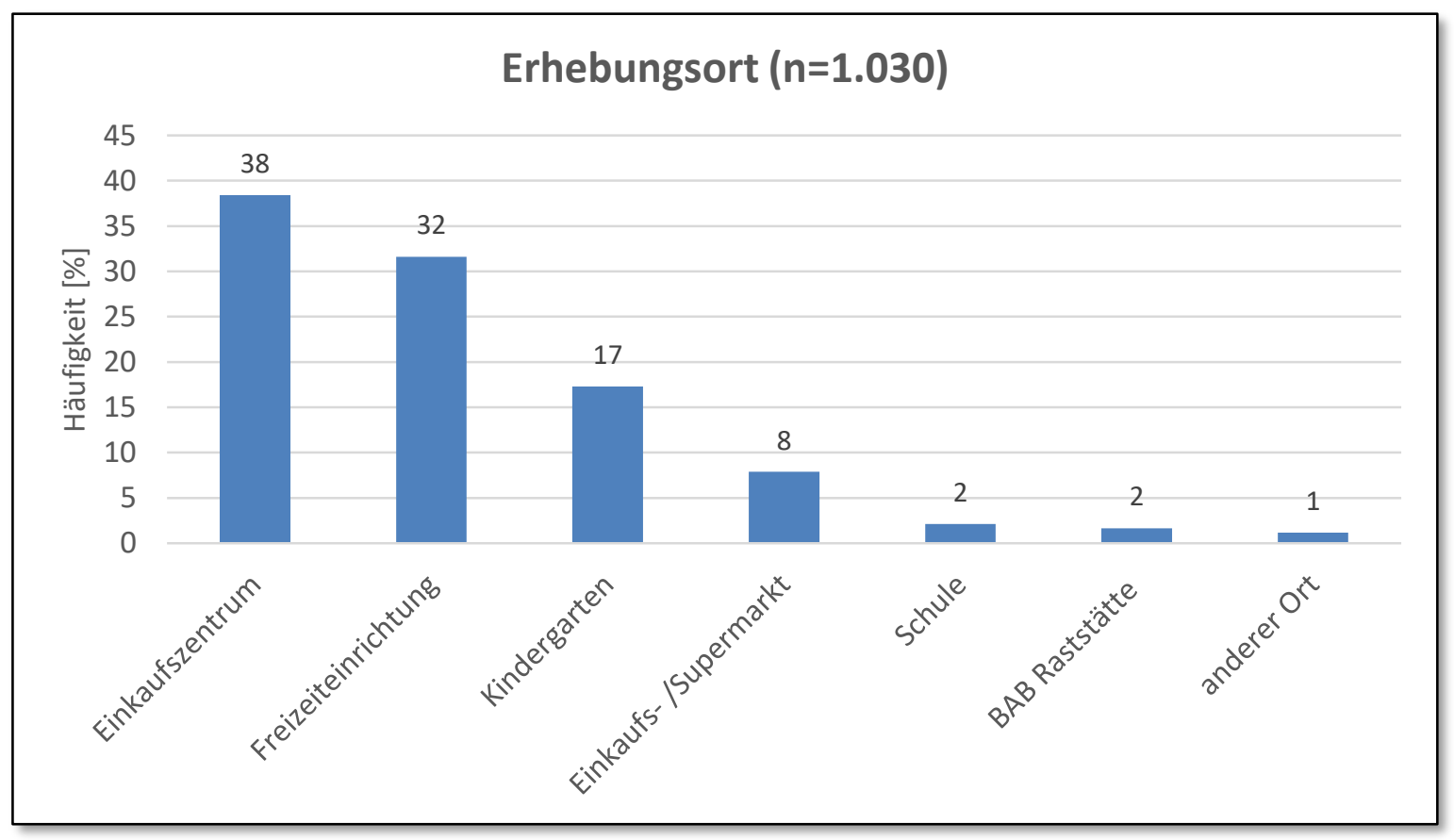

Abbildung 16: Erhebungsort in der Untersuchung

Die Erhebungen wurden an möglichst verschiedenen Orten durchgeführt, an denen mit Kindern im Fahrzeug gerechnet werden konnte. Ursprüngliche Zielstellung war dabei, möglichst eine Gleichverteilung der Befragungsorte zu erzielen. In der Praxis ließ sich das jedoch nicht umsetzen. Während an Orten wie Freizeiteinrichtungen und Einkaufszentren eine Befragung in der Regel gut durchgeführt werden konnte und bei den Eltern zumeist auch auf Akzeptanz traf, war dies in eher hektischen Situationen wie morgens vor der Schule oder vor der Kita kaum möglich. Diese Umfragen stellten sich als ausgesprochen zeitaufwändig heraus. Deshalb wurden insbesondere Schulen, bei denen ohnehin auch nur ein kleines Zeitfenster vor Schulbeginn für die Befragung zur Verfügung stand, im Verlauf der Studie nur noch selten als Befragungsort ausgewählt (Abbildung 16).

Kriterium für die Auswahl der Probanden war zunächst, dass sich die entsprechenden Personen zu einer gegebenen Zeit mit einem Fahrzeug an diesen Kontaktplätzen aufhielten und Kinder im Alter bis 12 Jahren mitführten. Damit wurde grundsätzlich angestrebt, eine Zufallsstichprobe zu ziehen und dabei alle KSS-Systemarten in die Untersuchung mit einzubeziehen. Zur Kodierung der Misuse-Formen diente der im Anhang (6.1) dargestellte Bogen. 
Die eingesetzten Beobachter/Interviewer hielten sich möglichst gleichmäßig in unterschiedlichen Segmenten der jeweiligen Befragungsgebiete auf und suchten im Wesentlichen ankommende Fahrzeuge auf. In der Regel wurde der Fahrer des Fahrzeugs durch die Beobachter/Interviewer angesprochen, anschließend das Untersuchungsanliegen erklärt. Wenn sich die angesprochenen Fahrer bereit erklärten, an der Erhebung teilzunehmen, erfasste bei ankommenden Fahrzeugen der eine Beobachter die allgemeinen Daten aus dem Beobachtungsbogen. Der zweite Beobachter überprüfte unterdessen, wie die Sicherung der Kinder im KSS sowie die KSS-Befestigungen im Pkw vorgenommen worden waren. Anschließend wurde entweder die Vor-Ort-Befragung jeweils für alle im Fahrzeug befindlichen Kinder durchgeführt oder der Termin für eine telefonische Nachbefragung innerhalb der nächsten Tage vereinbart. Die meisten der willigen Probanden entschieden sich für die telefonische Nachbefragung; sie stellen allerdings nur eine kleine Minderheit dar, denn insgesamt ist die Verweigererquote im Vergleich zu früheren Befragungen sehr hoch. Zwar war die Bereitschaft, sich einer Vor-Ort-Beobachtung zu unterziehen, durchweg positiv einzuschätzen, einer weiteren Befragung wollte aber nur eine Minderheit der Stichprobe zustimmen (siehe Kapitel 3.3).

Der Zeitbedarf pro Fahrzeug ohne weitere Vor-Ort-Befragung betrug durchschnittlich 15-20 Minuten und variierte je nach Anzahl der gesicherten Kinder im Fahrzeug. Unmittelbar nach jedem Fall prüften die Beobachter die Erhebungsbögen auf Vollständigkeit bzw. trugen fehlende Angaben nach.

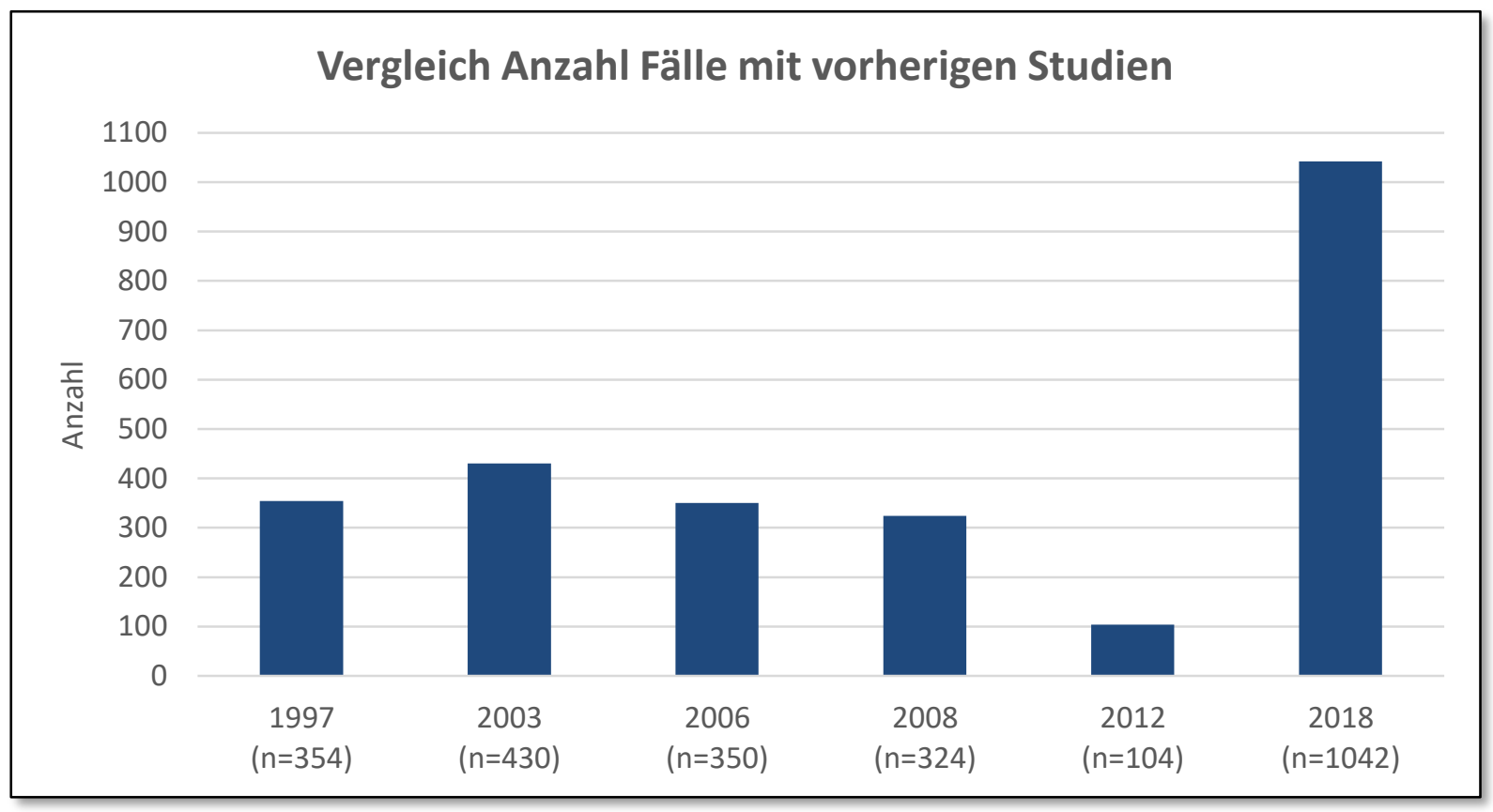

Abbildung 17: Vergleich der Fallanzahl mit vorherigen Studien [Langwieder, 1997; Hummel, 2003; Fastenmeier, 2006; Hummel, 2008; Casper, 2012] 
Im Vergleich zu den eingangs erwähnten früheren Studien konnte in der hier vorgestellten Felderhebung die Fallzahl deutlich gesteigert werden. Der Datensatz mit 1.042 gültigen und damit auswertbaren Einträgen erlaubt auch für spezielle Fragestellungen eine detaillierte Auswertung, die aufgrund der Fallzahl belastbare Ergebnisse mit sich bringt. 


\section{Ergebnisse der Felderhebungen}

In diesem Kapitel wird zunächst die Stichprobe in ihrer Gesamtheit beschrieben. Anschließend werden die Ergebnisse der Feldbeobachtung (s. Kapitel 3.2) vorgestellt. In Kapitel 3.3 werden dann die Resultate der Telefonbefragung gezeigt.

Wenn signifikante Unterschiede in den einzelnen Ausprägungen aufgetreten sind, wird darauf explizit hingewiesen.

\subsection{Beschreibung der Stichprobe}

Nachfolgender Überblick beschreibt die Stichprobe anhand von Daten zu den untersuchten Kindern, den erfassten Fahrzeugen, den mitfahrenden Insassen und den genutzten Kindersitzen. Basis dieser Beschreibung sind für die beiden Bereiche Insassen sowie Fahrzeuge die in den Felderhebungen erfassten Befragten $(n=721)$, für die beiden Bereiche Kinder und Kindersitze hingegen die in den Fahrzeugen befindlichen Kinder in Kindersitzen $(n=1.042)$; eine davon abweichende Stichprobengröße aufgrund fehlender Angaben („missing“) wird jeweils ausgewiesen.

\section{Insassen im Fahrzeug}

Die Anzahl der Insassen in den untersuchten Fahrzeugen reichte von zwei bis sechs Personen. In über $60 \%$ der untersuchten Fahrzeuge befanden sich zwei oder drei Personen (vgl. Abbildung 18).

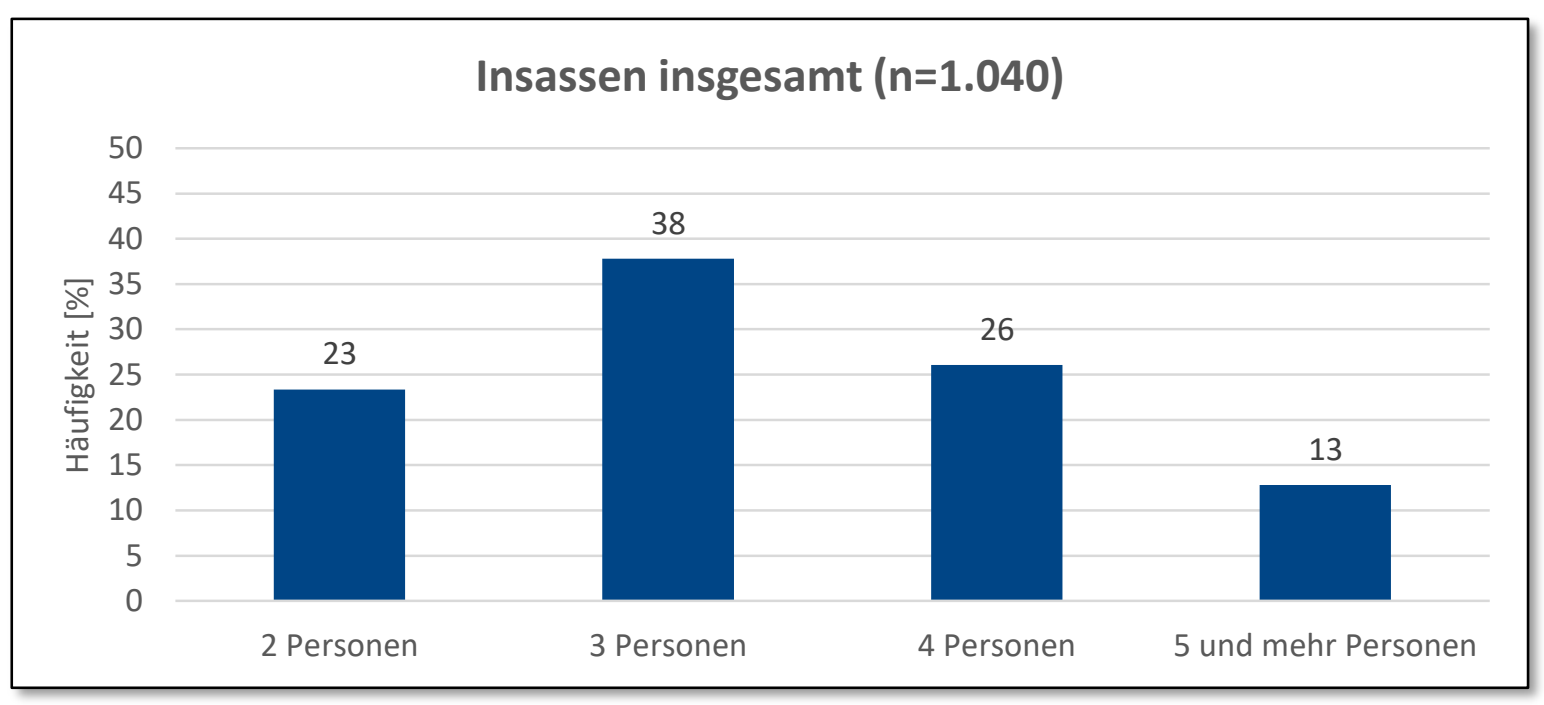

Abbildung 18: Verteilung der Fahrzeuginsassen in den erfassten Fahrzeugen 
Die Anzahl der Kinder unter 12 Jahren (also Kinder, die in einem Kindersitz zu sichern sind) variierte in den untersuchten Fahrzeugen von einem Kind bis vier Kindern. In mehr als $80 \%$ der Fahrzeuge handelte es sich um ein einzelnes Kind oder um zwei Kinder.

\section{Kinder unter 12 Jahren}

Von den insgesamt in den Erhebungen erfassten 1.042 Kindern waren 47,3\% weiblich und $52,7 \%$ männlich. Das Alter der Kinder reichte von einem Monat bis 12 Jahre (drei Fälle wurden ergänzend erhoben, bei denen die Kinder 12 Jahre oder älter waren und in einem KSS saßen). Abbildung 19 zeigt die Altersverteilung der Kinder. Diese Altersverteilung der Kinder spiegelt sich zum Teil auch in den Angaben zu ihrer Größe und ihrem Gewicht wider (vgl. Abbildung 20 und Abbildung 21).

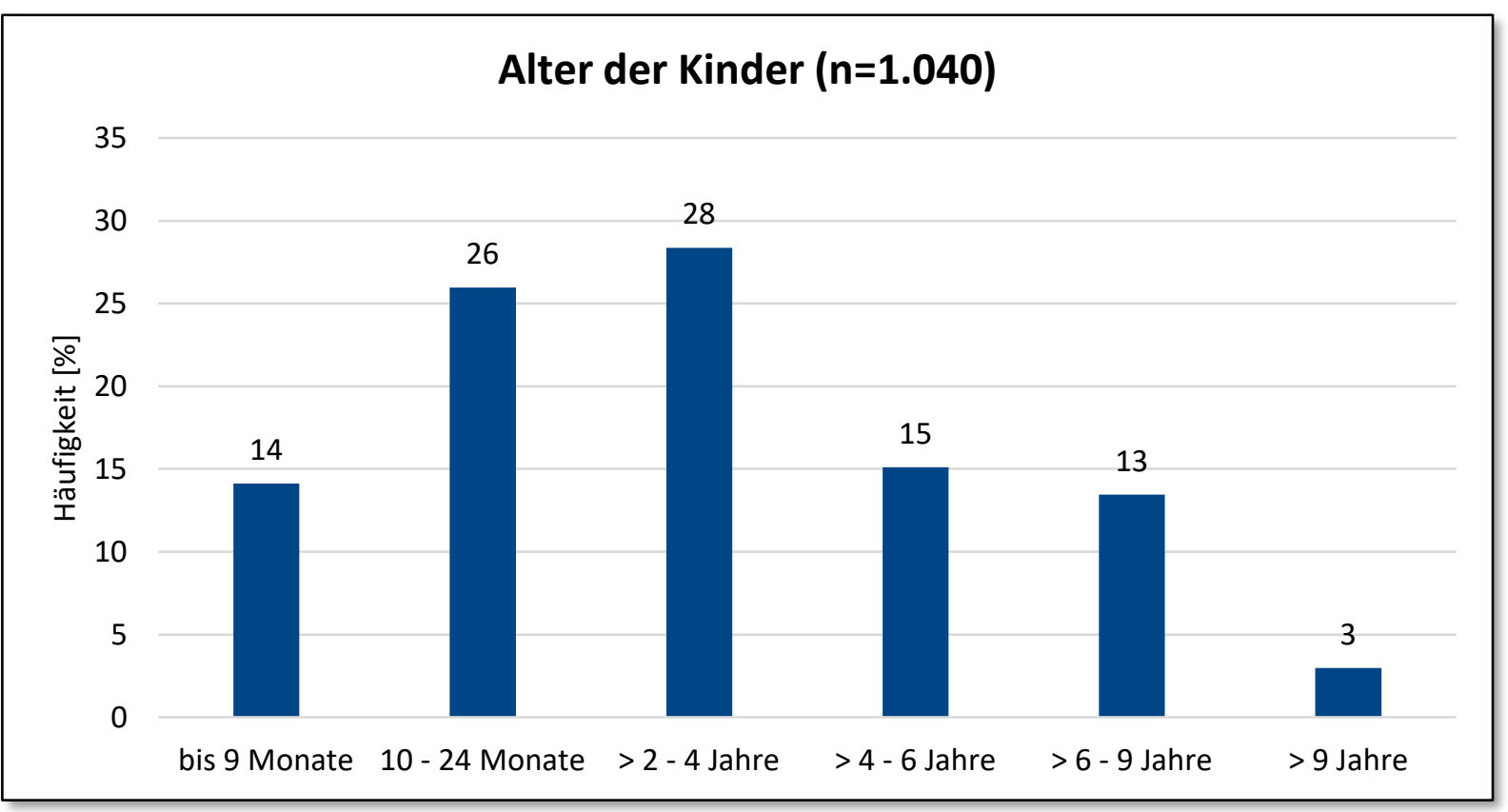

Abbildung 19: Altersverteilung der Kinder unter 12 Jahren 


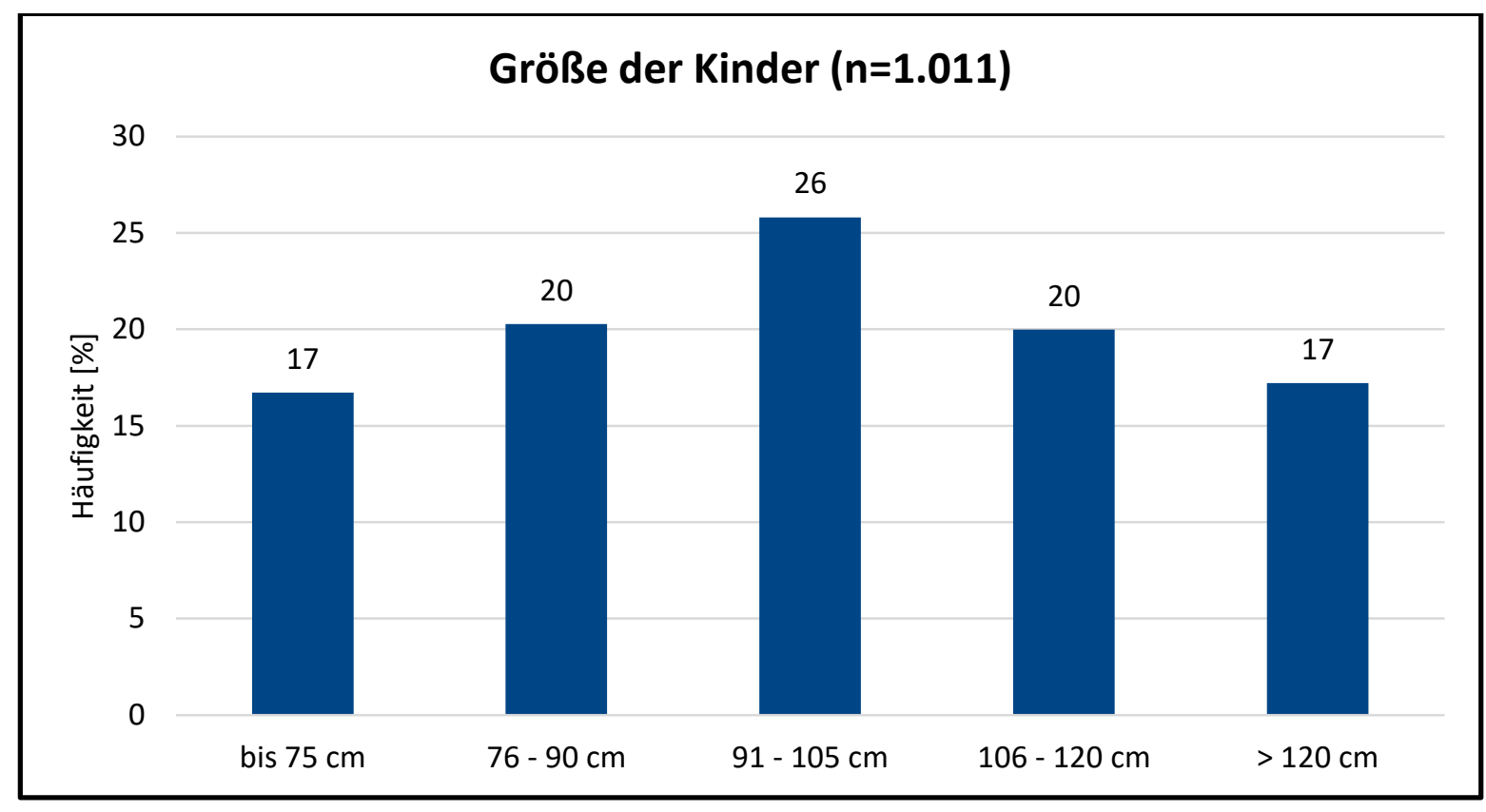

Abbildung 20: Größe der Kinder

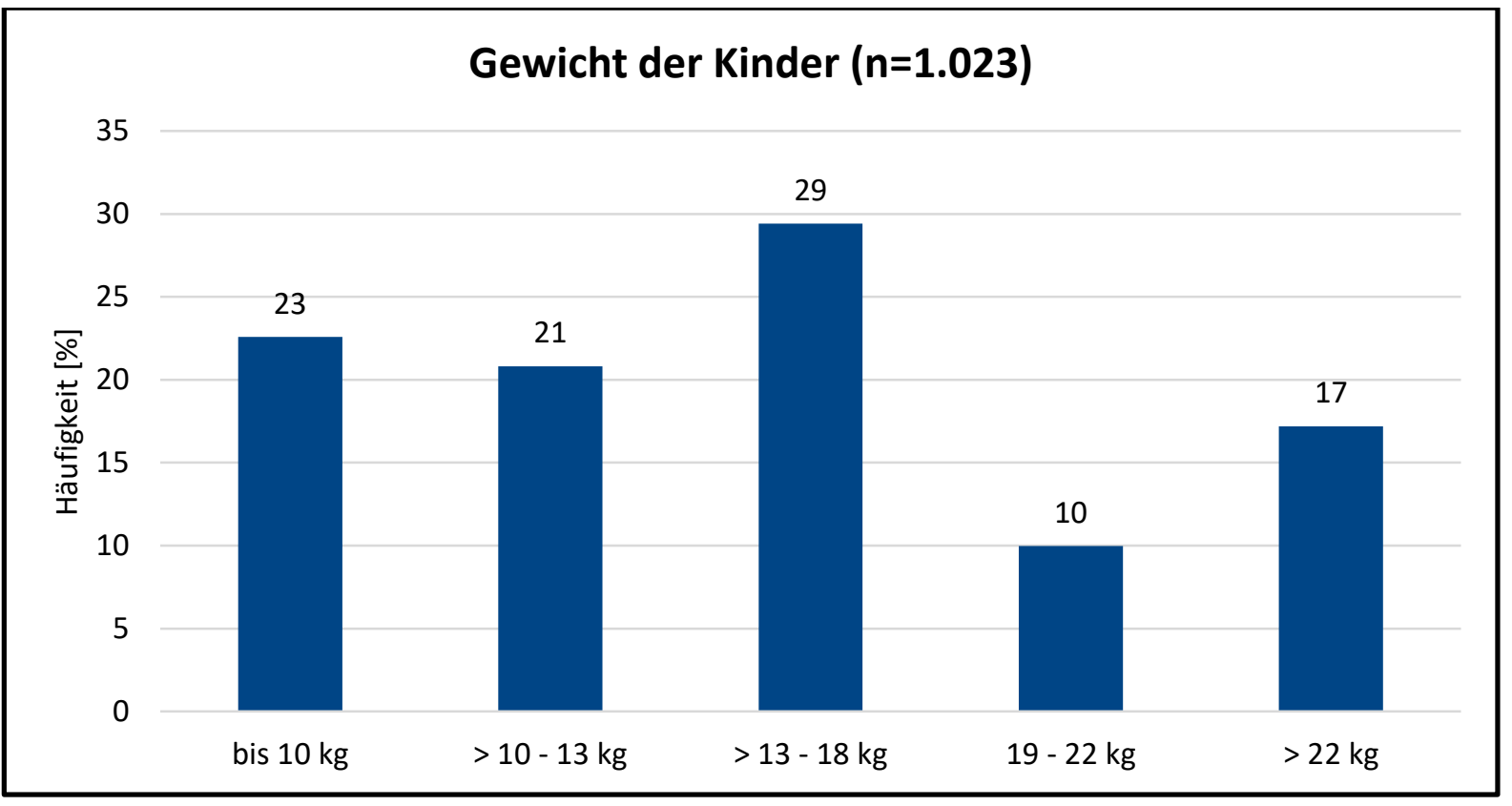

Abbildung 21: Gewicht der Kinder

Die überwiegende Mehrheit der Kinder war auf der Rückbank hinter Fahrer- und Beifahrersitz positioniert, am häufigsten rechts hinten (50,3\%); auf dem Beifahrersitz befanden sich $13 \%$ und auf der 3. Sitzreihe lediglich 1,5\% der Kinder (vgl.Abbildung 22). 
Wie Abbildung 28 zeigt, werden Gruppe 0/0+-Systeme fast ausschließlich rückwärtsgerichtet, Gruppe 1 sowie 2/3 Systeme nahezu ausschließlich vorwärtsgerichtet verwendet.

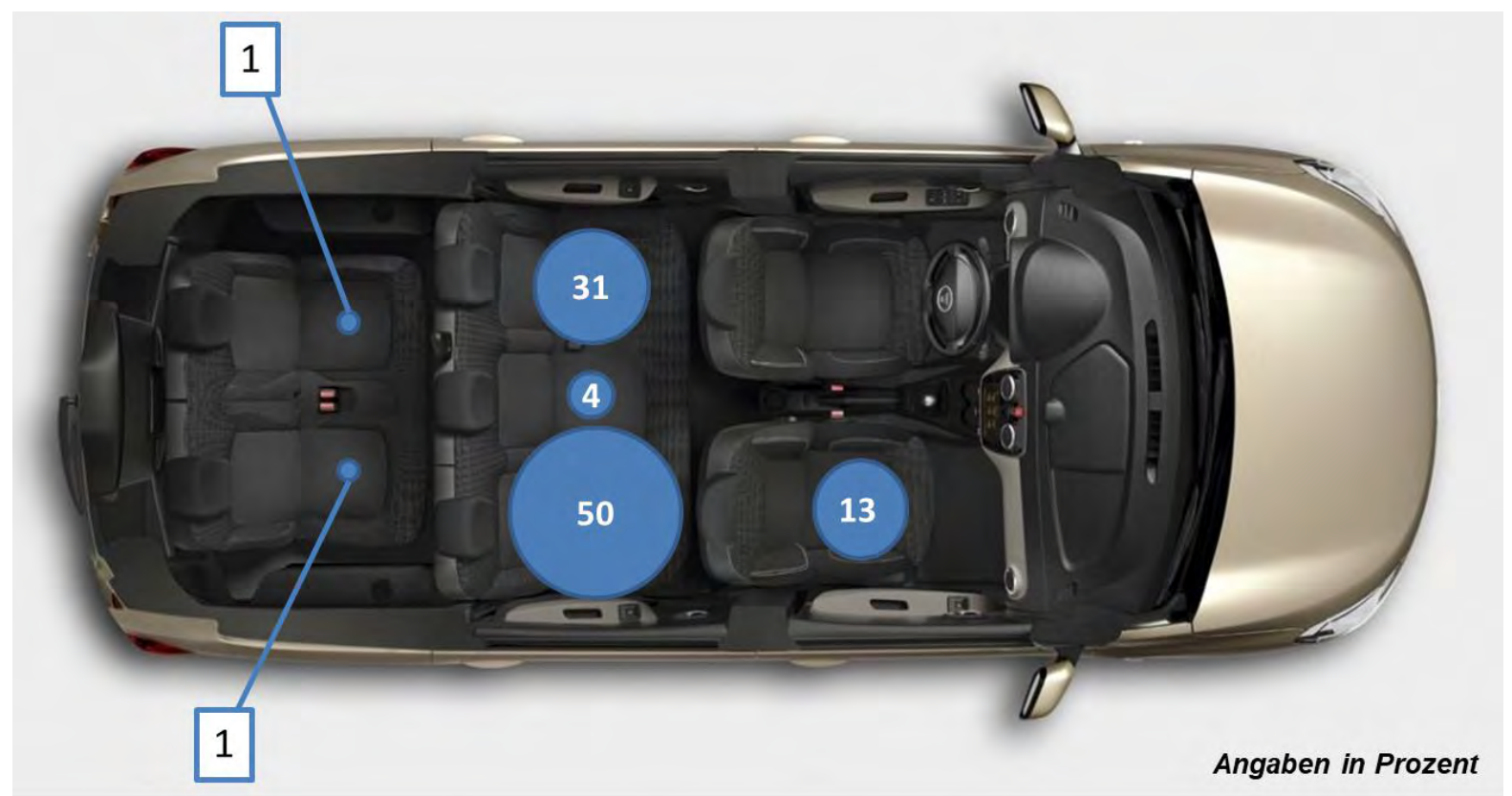

Abbildung 22: Sitzposition der Kinder ( $n=1.036)$ 


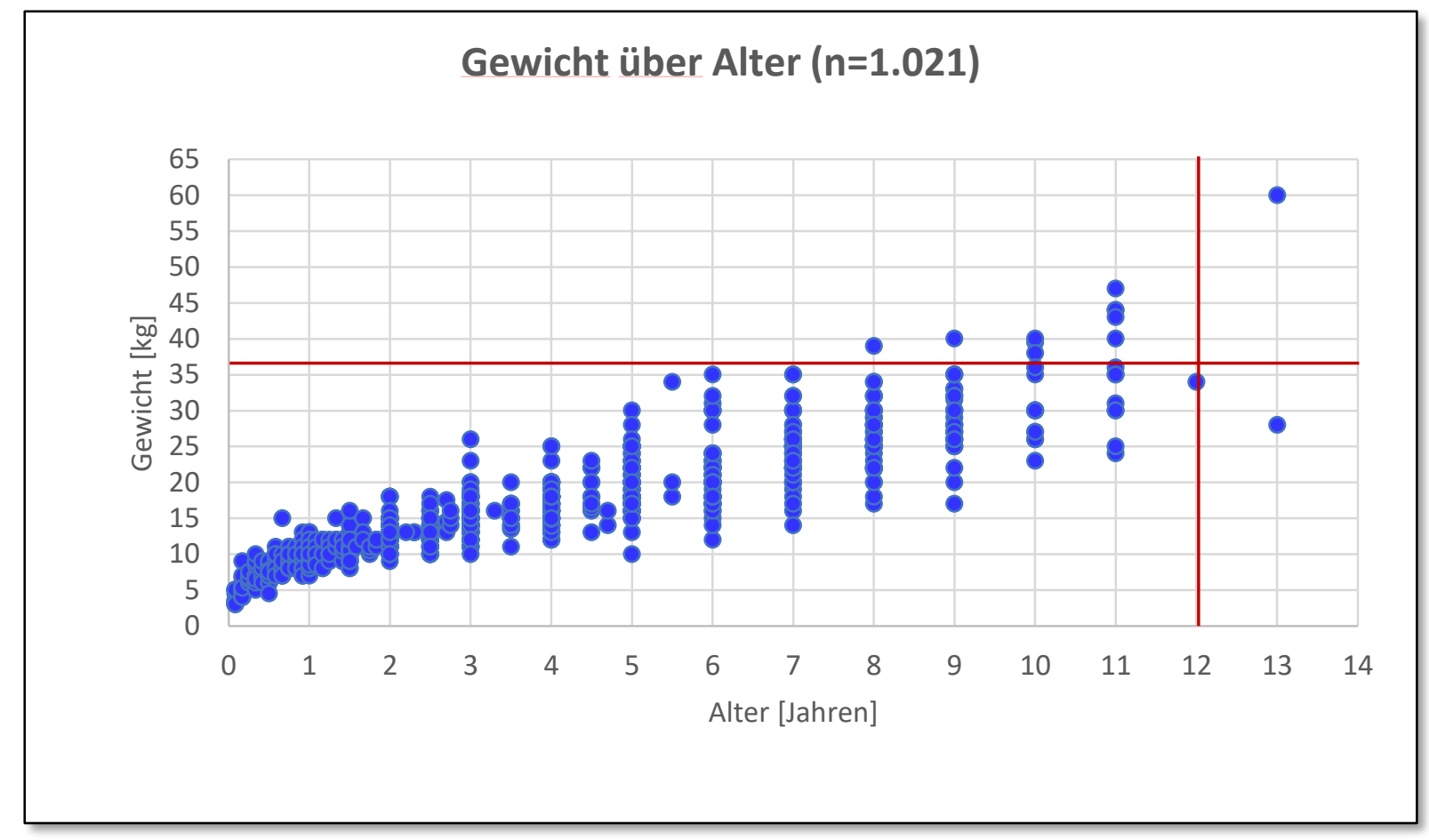

Abbildung 23: Gewicht über Alter

In Abbildung 23 ist die Alters- und Gewichtsverteilung der Kinder dargestellt, die in dieser Studie erfasst wurden. Bemerkenswert dabei ist, dass der Anteil der Kinder, die jünger als 12 Jahre und gleichzeitig schwerer als $36 \mathrm{~kg}$ sind, im Vergleich zu vorherigen Studien zugenommen hat (vgl. Abbildung 23).

Weitere Abbildungen zur Größen- und Gewichtsverteilung der untersuchten Kinder, sind in Anhang 6.5 zu finden.

\section{Soziodemographische Merkmale der Interviewpartner}

Wie erwähnt, wurden in der Regel die Fahrer des erfassten Fahrzeugs - dies waren in 63,3 \% der Fälle Frauen - angesprochen. Bei $58 \%$ der Interviewpartner handelte es sich um die Mutter des Kindes/der Kinder, bei 33,7\% um den Vater, bei 5,4\% um ein Großelternteil und bei 2,9\% um andere Verwandte oder um Bekannte. Im Vergleich zu früheren Studien hat damit der Anteil der Frauen deutlich abgenommen.

Abbildung 24 gibt die Altersverteilung der Interviewpartner wieder. Der Großteil der Altersgruppen (von $<30$ bis zu 60 Jahren) ist relativ gleichmäßig vertreten (von $21-30 \%$ ). Befragte mit mehr als 60 Jahren hatten einen Anteil von lediglich 4\%. 


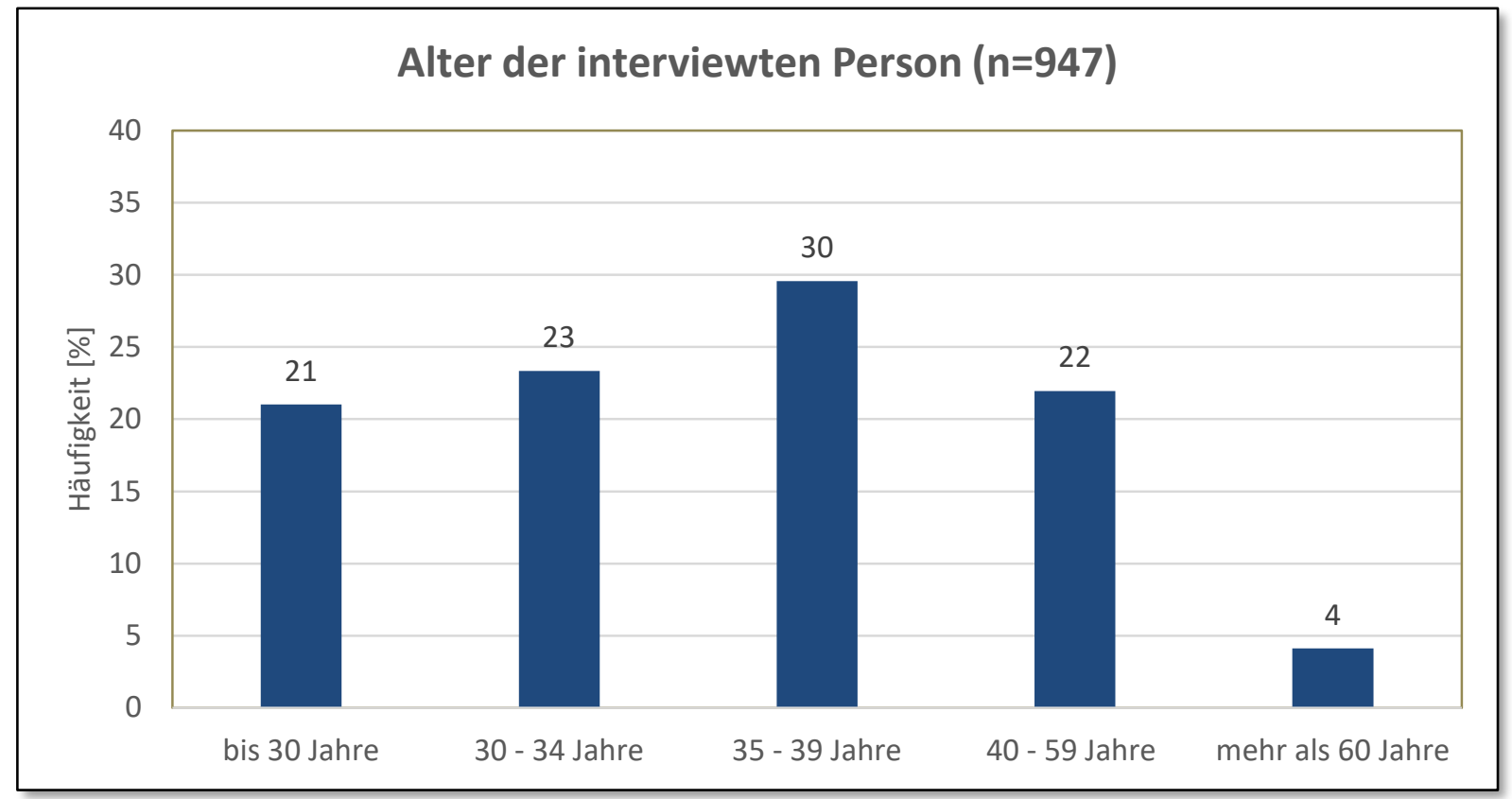

Abbildung 24: Alter der interviewten Person

Über $85 \%$ der befragten Personen waren deutsche Staatsbürger, der Anteil der Ausländer lag entsprechend bei rund $15 \%$. Zwischen beiden Gruppen gab es hinsichtlich der Sicherungsqualität signifikante Unterschiede, was in den folgenden Kapiteln an verschiedenen Stellen aufgezeigt wird.

\section{Fahrzeugmerkmale}

Bei den in den Erhebungen am häufigsten erfassten Fahrzeugarten handelte es sich mit jeweils etwa einem Drittel um Kleinwagen, Kombis und Großraumwagen bzw. Vans (vgl. Abbildung 25). Auffallend ist der relativ hohe Anteil von Kleinwagen. Das Alter der Fahrzeuge verteilt sich ebenfalls relativ gleichmäßig, etwas überraschend mag auch der relativ hohe Anteil von Fahrzeugen mit einem Alter von mehr als 9 Jahren $(33,2 \%)$ erscheinen (vgl. Abbildung 26). 


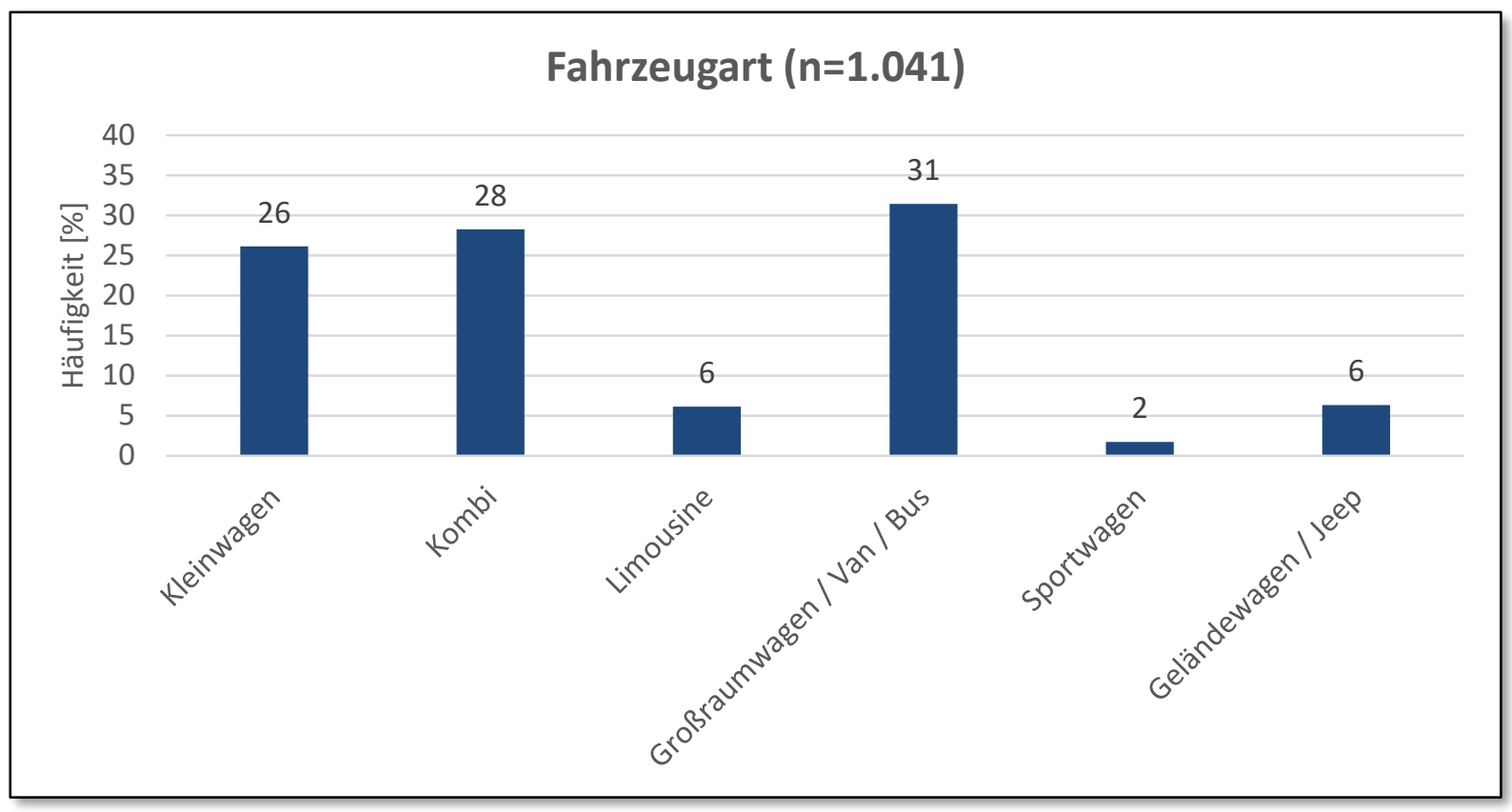

Abbildung 25: Erfasste Fahrzeugarten

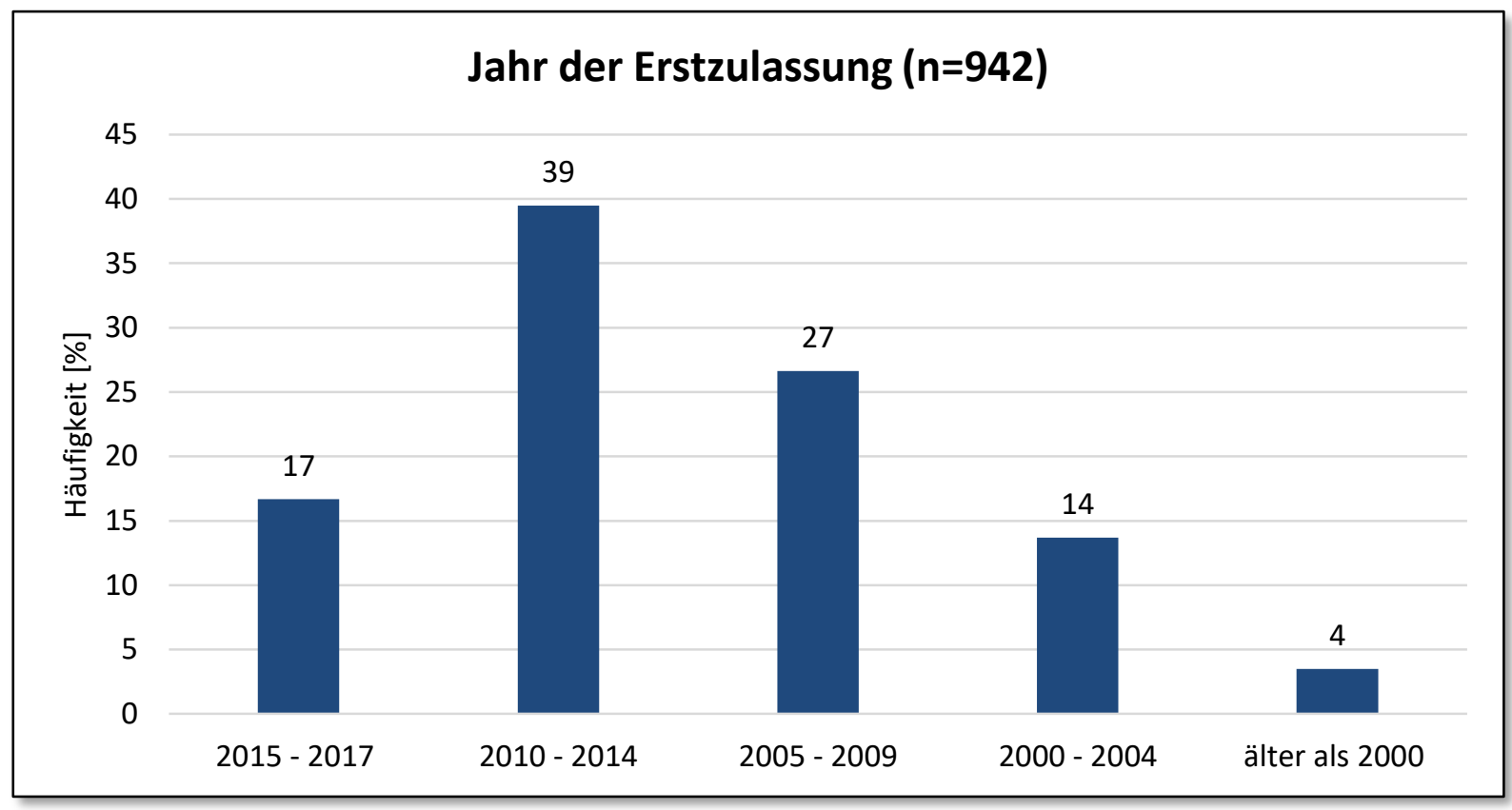

Abbildung 26: Alter der Fahrzeuge 


\section{Verwendete Schutzsysteme}

Am häufigsten waren die Systemgruppen $2 / 3$ mit 45,3, \% sowie 1 mit 35,7 \% vertreten; auf Kindersitze der Gruppe 0/0+ entfielen $19 \%$.

90,4 \% der verwendeten Schutzsysteme entsprachen der ECE-Norm 44.04, 7,9 \% der ECENorm 44.03. Nur 0,3\% entfällt auf die veraltete ECE-Norm 44.02 und 1,4\% auf die neue Norm ECE-R 129.

Befestigt waren die meisten KSS (79,6 \%) mit einem 3-Punkt-Gurt-Automatik-System, in $20 \%$ wurde ein ISOFIX-System verwendet, in nur 4 Fällen handelte es sich um integrierte KSS. Hier lässt sich also im Vergleich zu früheren Studien eine ebenso deutliche wie auch erfreuliche Hinwendung zu ISOFIX konstatieren (Abbildung 27).

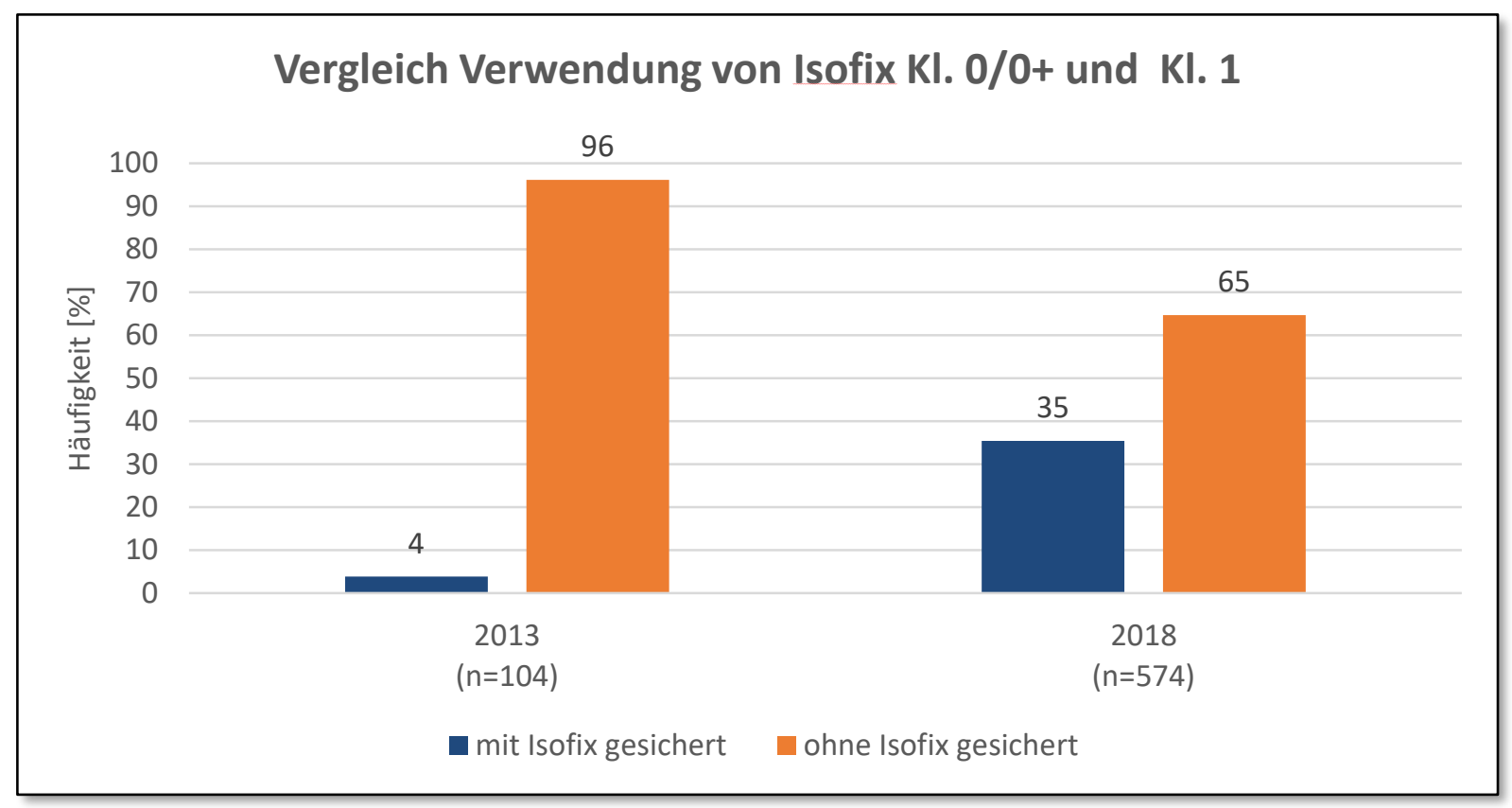

Abbildung 27: Verwendung von ISOFIX in KI.0/0+ und KI.1

Von den in einem Klasse 1-Sitz gesicherten Kindern saßen $81 \%$ in einem Sitz mit integriertem 5-Punkt-Gurtsystem, $19 \%$ der Kinder saßen in einem Sitz mit einem integrierten Fangkörper. In der Systematik dieser Studie wurden KSS mit integriertem Fangkörper immer dann als Gruppe 1-Sitz gewertet, wenn der Fangkörper tatsächlich auch genutzt wurde. War das nicht der Fall, wurde der Kindersitz dann entsprechend als Klasse 2/3-Sitz gezählt. 
Von den genutzten Kindersitzen waren 78,4 \% vorwärts, 21,4 \% rückwärts eingebaut; ein KSS wurde auf einer Sitzbank entgegen der Fahrtrichtung genutzt (Abbildung 28). Während rückwärtsgerichtete Kindersitze der Klasse 1 zwar sehr selten aber dennoch zunehmend häufiger in Deutschland zu finden sind, handelt es sich bei vorwärts gesicherten Babyschalen (Klasse $0 / 0+$ ) in jedem Fall um schwere Fehlbenutzung. Kindersitze dieser Klasse sind ausschließlich für die rückwärtsgerichtete Beförderung von Kindern zugelassen. Eine Sicherung in Fahrtrichtung führt im Fall eines Frontalaufpralls mit hoher Wahrscheinlichkeit zu Verletzungen.

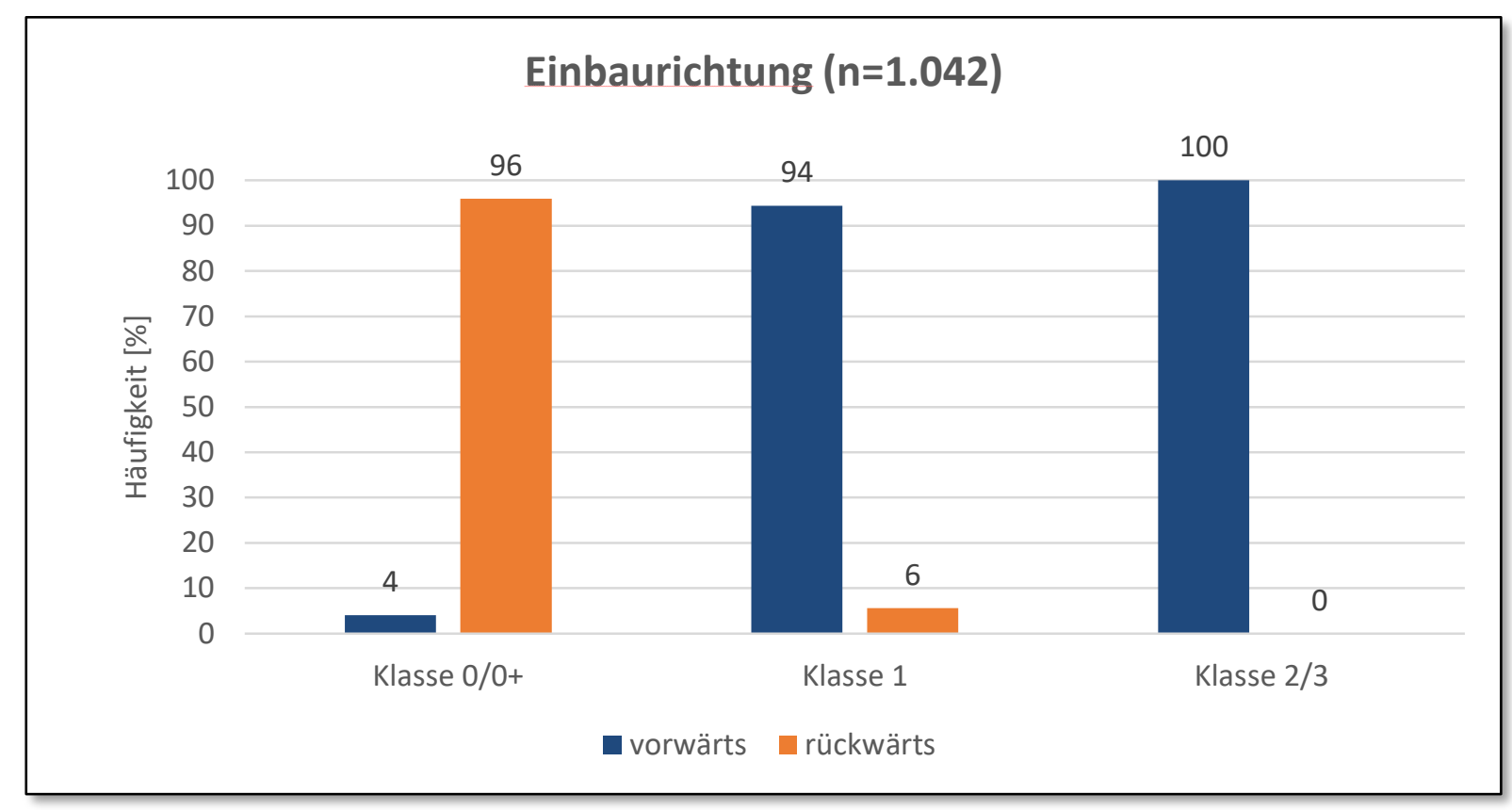

Abbildung 28: Einbaurichtung

\section{Merkmale der Fahrt}

Entsprechend der Anteile der gewählten Kontaktplätze handelte es sich beim Zweck der unternommenen Fahrt am häufigsten um Einkaufsfahrten, gefolgt von Freizeitfahrten und Fahrten, um Kinder zum Kindergarten/zur Schule zu bringen oder sie wieder abzuholen; andere Fahrtzwecke spielten eine lediglich untergeordnete Rolle. 


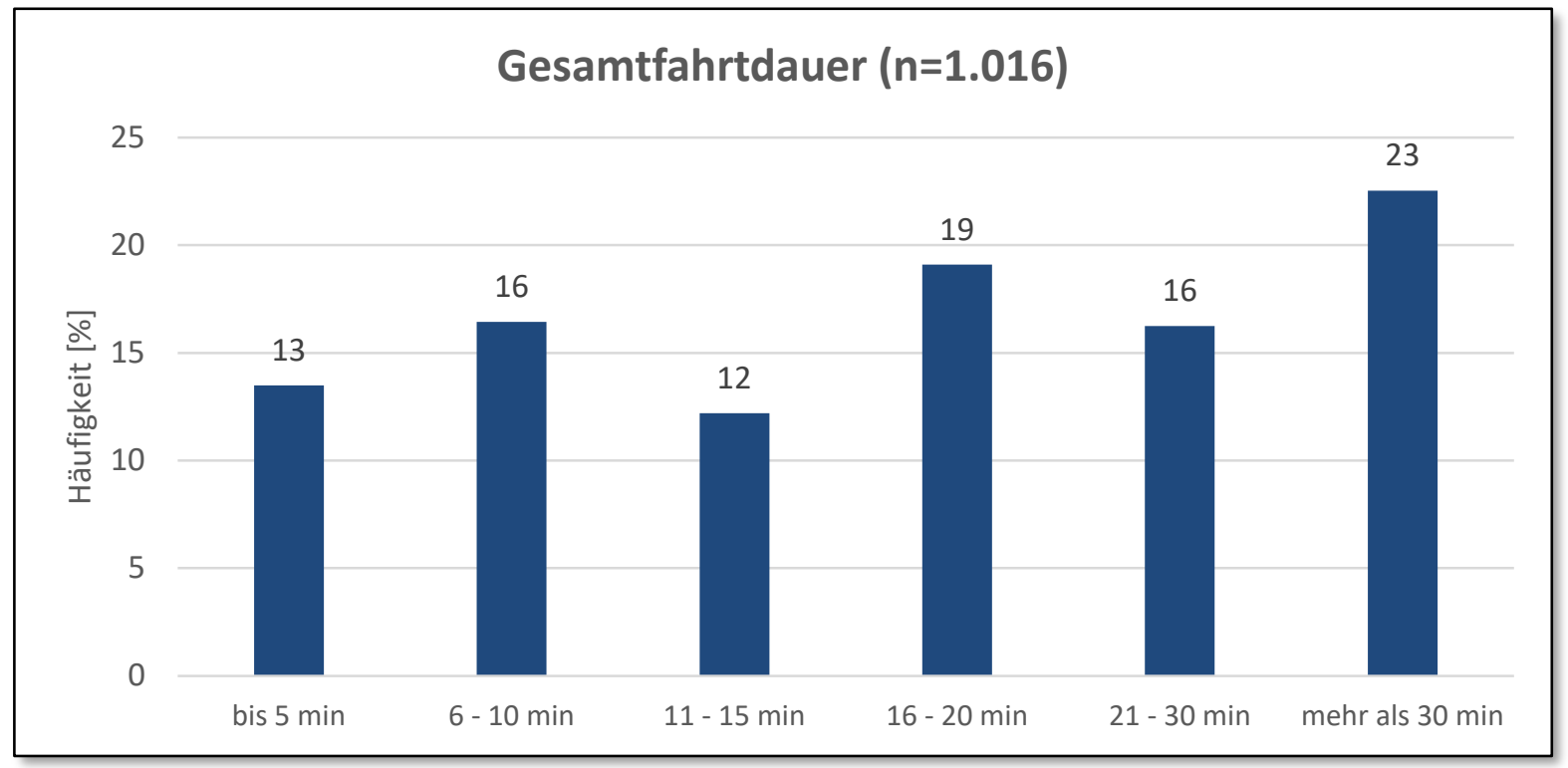

Abbildung 29: Fahrtdauer

Untersucht man die Gesamtdauer der Fahrt ${ }^{1}$, dann zeigt sich - wie auch schon in früheren Studien festgestellt -, dass es sich überproportional oft um kurze Fahrten mit bis zu 5 min oder 6 bis 10 min handelte (ca. $30 \%$ ). Auch die längeren Fahrtdauern (21 bis 30 min und länger als $30 \mathrm{~min}$ ) entsprechen mit Anteilen von knapp $40 \%$ früheren Ergebnissen. Abbildung 29 gibt einen Überblick zur Fahrtdauer.

\subsection{Ergebnisse der Misuse-Beobachtungen}

In diesem Abschnitt des Berichts geht es im Wesentlichen um die Auswertung des Beobachtungsteils der Studie. Insbesondere die verschiedenen Formen von Misuse, dessen Schwere und die Umstände, die den Fehlgebrauch wahrscheinlicher machen, werden anhand der erhobenen Daten analysiert und bewertet. Dieser Abschnitt gliedert sich in die Teile „Misuse allgemein“, „Installation des KSS“, „Sicherung des Kindes“, „Sonstige Fehler“ und „weitere Beobachtungen“.

\section{Untersuchung Misuse allgemein}

Von den insgesamt 1.042 untersuchten Kindern waren 52,4 \% korrekt und 47,6 \% fehlerhaft gesichert. Damit liegt die Misusequote im Vergleich zu bisherigen Studien erstmals unter

${ }^{1}$ Gemeint ist hier die Dauer von Start bis zum Ende der Fahrt, unabhängig vom Zeitpunkt der Befragung 
$50 \%$ (die Studie aus dem Jahr 2012 ist hier als Vergleich aufgrund der geringen Fallzahl ungeeignet) (Abbildung 30). Es zeigt sich also, dass der Anteil der fehlerhaften Nutzung von Kindersitzen deutlich zurückgegangen ist. Auch wenn die Ursachen für diese positive Entwicklung erst im Folgenden genauer herausgearbeitet werden sollen, zeigt sich schon jetzt, dass die in den letzten Jahren angestrebten Entwicklungen eine positive Wirkung entfaltet haben.

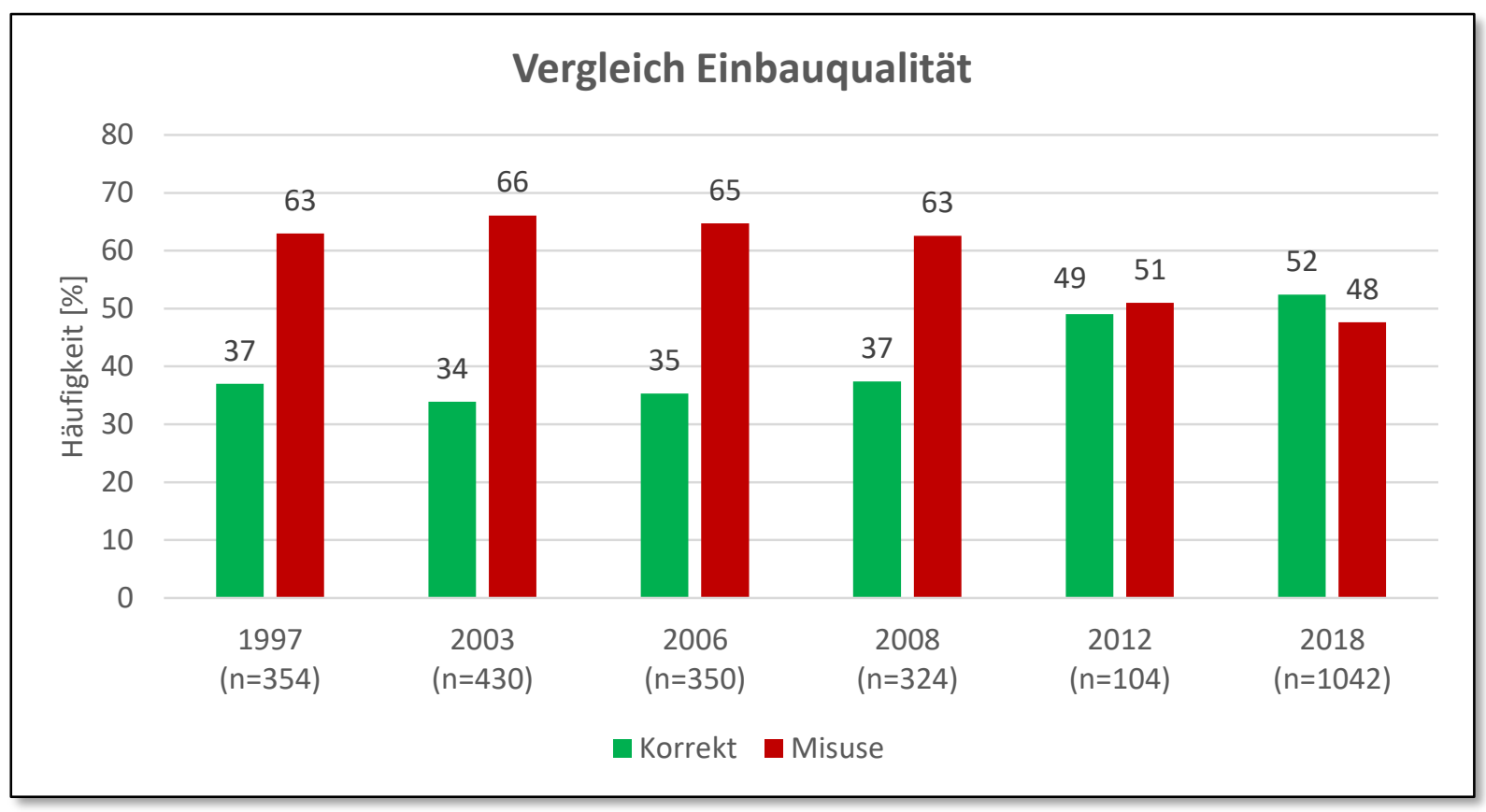

Abbildung 30: Vergleich der Misusequote über die Zeit [Langwieder, 1997; Hummel, 2003; Fastenmeier, 2006; Hummel, 2008; Casper, 2012]

Aufgeschlüsselt auf die einzelnen KSS-Klassen ist die Misusequote sehr unterschiedlich. Die fehlerhafte Verwendung von Kindersitzen tritt in den Klassen $0 / 0+$ und 1 signifikant häufiger auf als in der Klasse $2 / 3$ (vgl. Abbildung 31) ( $p=0,004)$. Das entspricht auch den Resultaten früher Studien. Ursächlich dafür ist im Wesentlichen, dass die Sicherung in einem Klasse 2/3-Sitz deutlich einfacher ist. Hier werden Kind und Kindersitz gemeinsam mittels Fahrzeuggurt auf ähnliche Weise im Fahrzeug gesichert, wie das bei Erwachsenen der Fall ist. Bei KSS der anderen beiden Klassen ist für die Sicherung des Kindes in der Regel ein eigenes Gurtsystem zu nutzen, die Installation des Sitzes im Fahrzeug hängt individuell von der Gestaltung des Sitzes und ganz grundsätzlich von der Art der Anbindung ans Fahrzeug ab. Das kann entweder der gewöhnliche 3-Punkt-Gurt sein oder das ISOFIXSystem. 


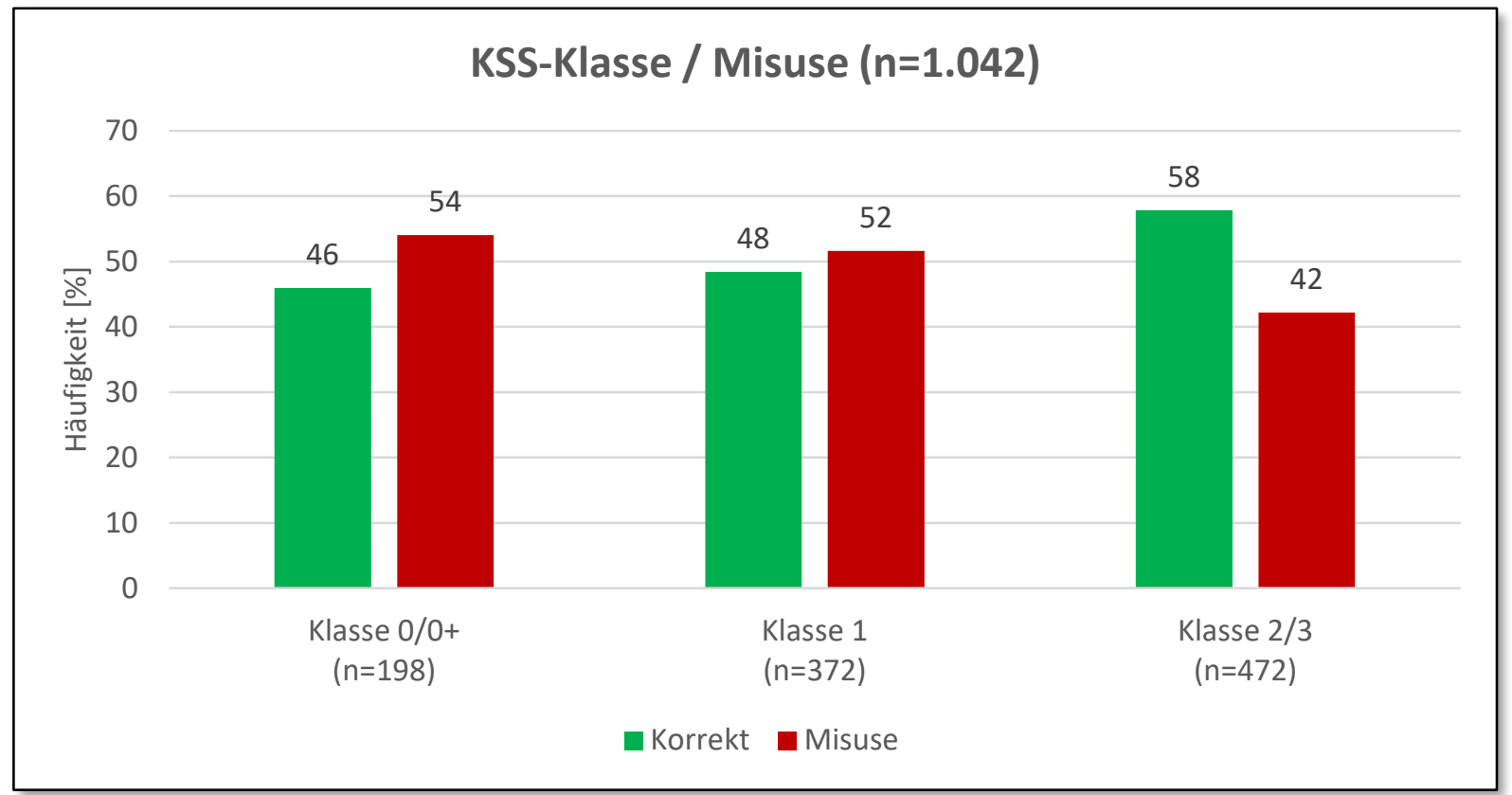

Abbildung 31: Misusequote über KSS-Klassen

Die Misuseschwere ist über die Stufen „leicht", „mittel“ und „schwer" nicht gleich verteilt. Mehr als die Hälfte des gesamten beobachteten Misuse، wurde als schwer bewertet, $35 \%$ als mittel und $15 \%$ als leicht (Abbildung 32). Im Vergleich zu vorherigen Studien muss damit zur Kenntnis genommen werden: Die Misusequote ist insgesamt zwar deutlich zurückgegangen, aber die Schwere des festgestellten Misuse ist angestiegen (s. Abbildung 33). Es scheint so zu sein, dass fortschrittliche Entwicklungen bei modernen KSS dafür sorgen, dass einige gängige Misuseformen recht gut vermieden werden können. Kommt es dann aber doch zu Fehlbenutzungen, sind diese vergleichsweise schwerwiegend. Diese These wird im Folgenden anhand verschiedener Auswertungen überprüft. 


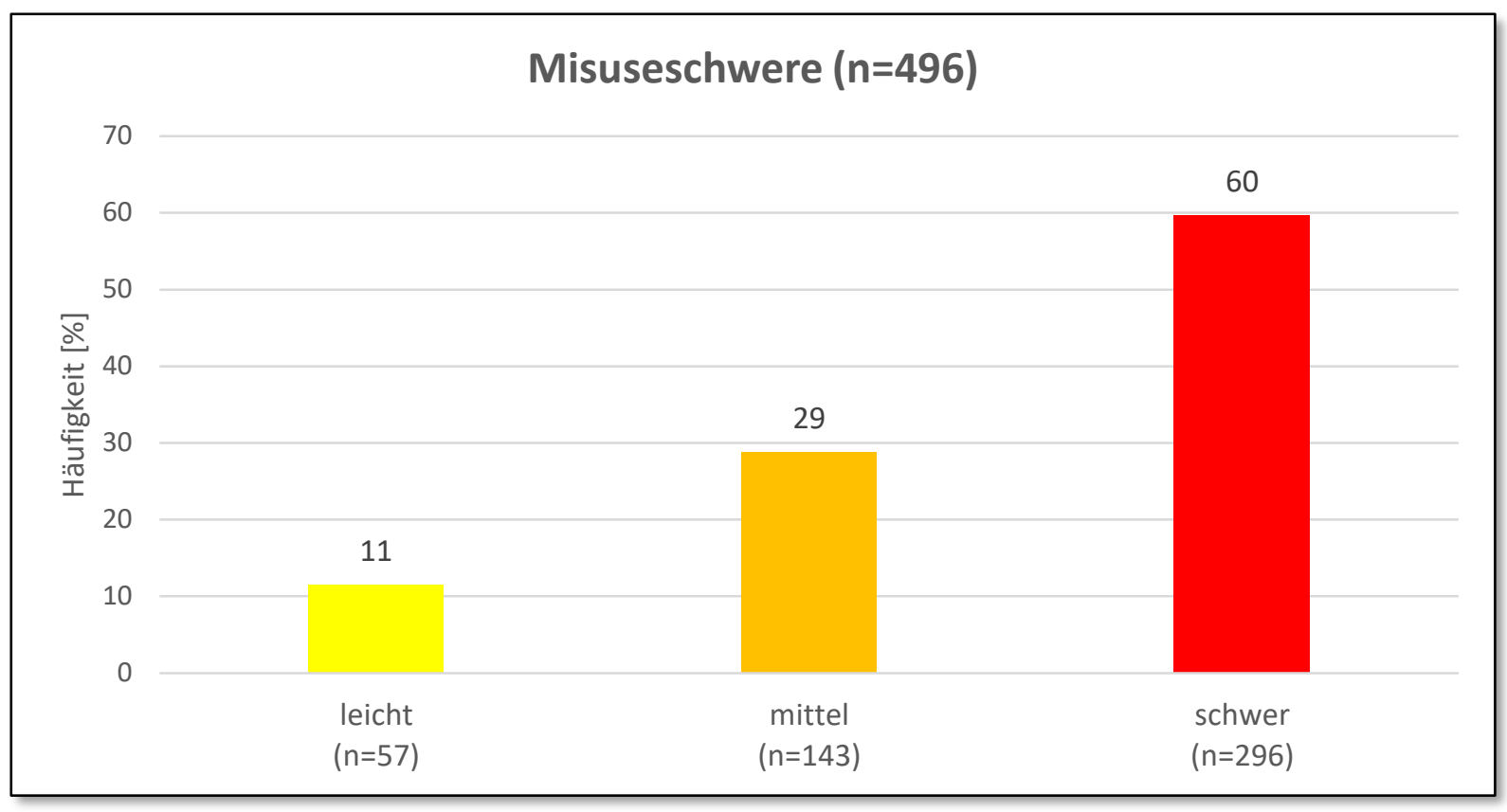

Abbildung 32: Misuseschwere

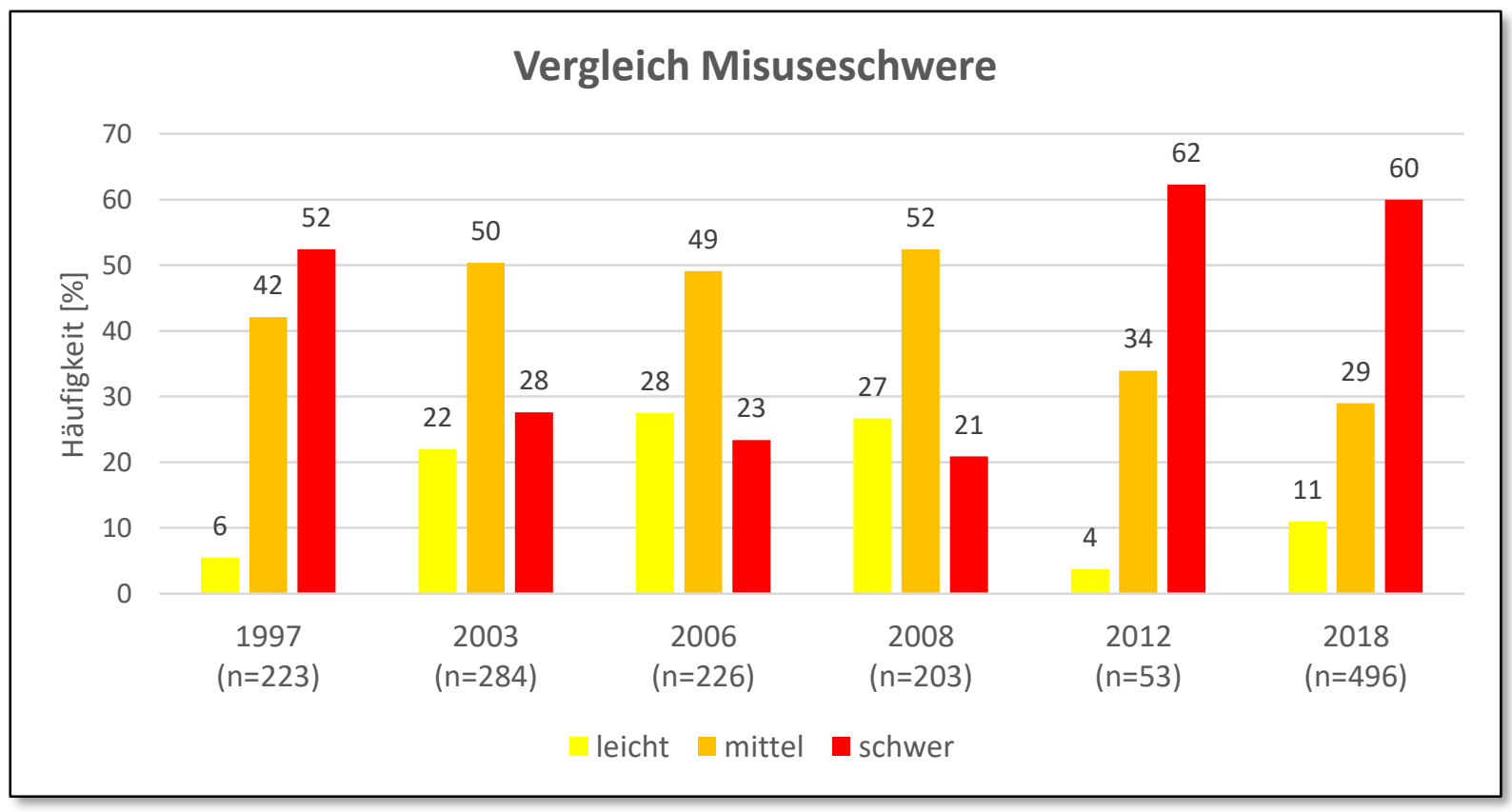

Abbildung 33: Vergleich der Misuseschwere über die Zeit

[Langwieder, 1997; Hummel, 2003; Fastenmeier, 2006; Hummel, 2008; Casper, 2012]

Im Folgenden werden verschiedene Faktoren untersucht, die möglicherweise das Auftreten von Misuse begünstigen. So zeigt sich zum Beispiel, dass schlechtes Wetter mit 
Niederschlag die Misusequote signifikant erhöht (Abbildung 34) $(p<0,001)$. Es liegt die Annahme nahe, dass bei Regenwetter der Sicherungsvorgang sehr schnell und entsprechend ungenau durchgeführt wird und dadurch die Kinder schlechter gesichert werden.

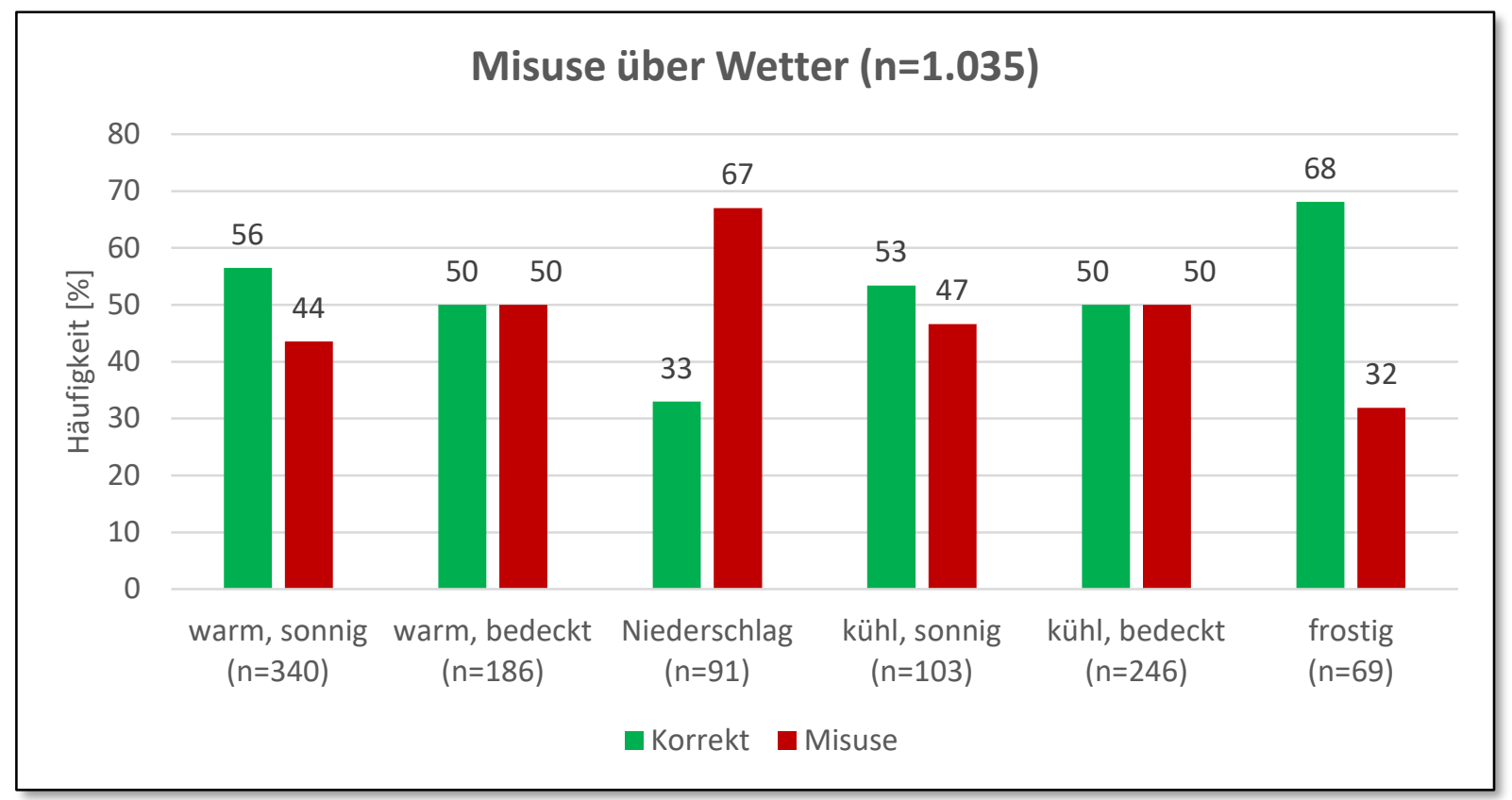

Abbildung 34: Misuse über Wetter

Die Fahrtdauer beeinflusst die Misusequote ebenfalls signifikant. Wie schon in früheren Studien zeigt sich, dass bei kurzen Fahrten bis zu einer Dauer von 10 Minuten deutlich mehr Fehler auftreten als bei längeren Fahrten (Abbildung 35) $(p=0,013)$. Offensichtlich ist nach wie vor die Annahme verbreitet, dass eine angemessene Sicherung bei kurzen Fahrten weniger wichtig ist. Diese Beobachtung kann auch immer wieder bei der Gurtanlegequote erwachsener Insassen gemacht werden. 


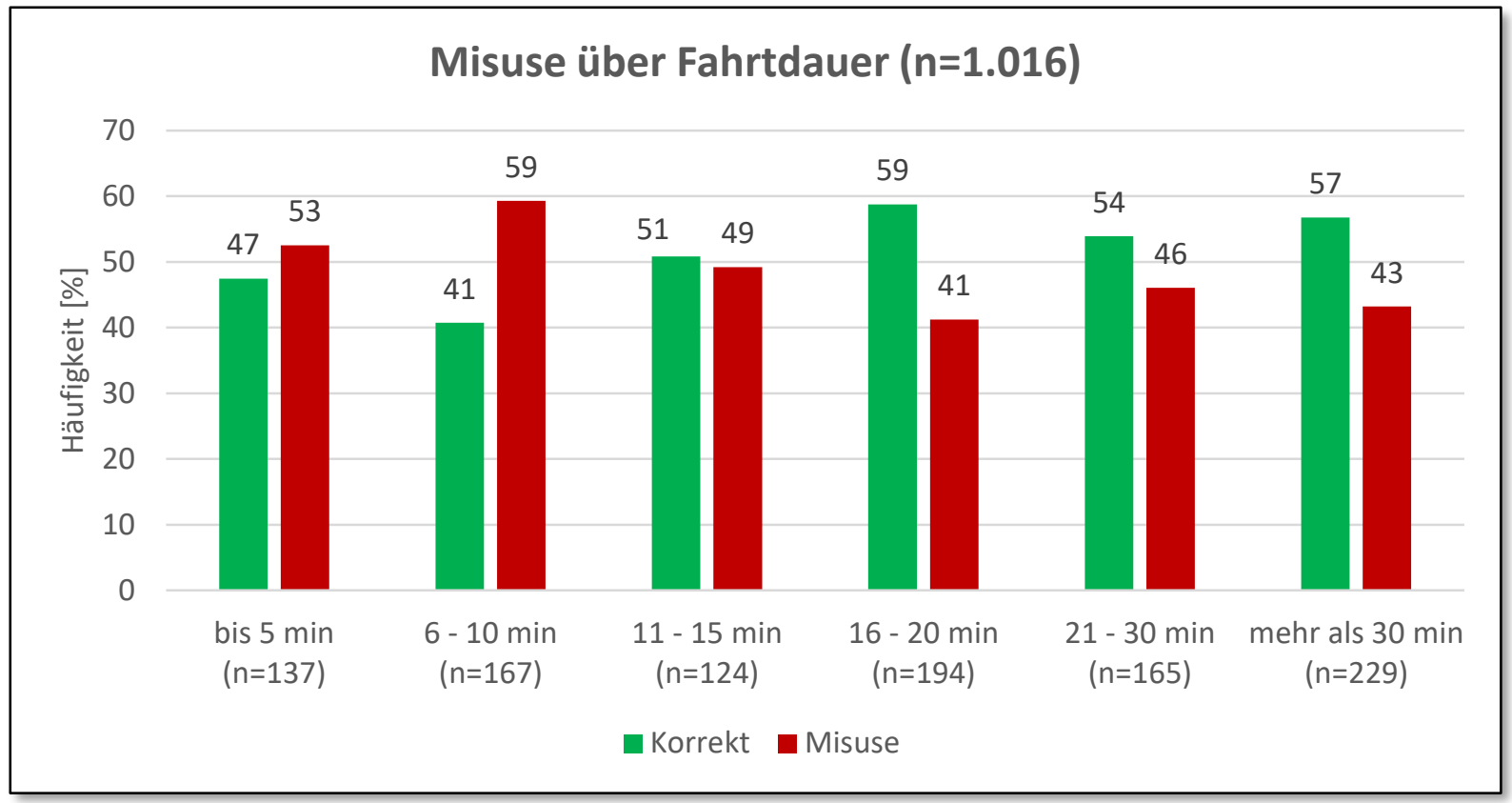

Abbildung 35: Misuse über Fahrtdauer

Als ebenfalls signifikant konnte der Zusammenhang zwischen Fahrtzweck und Misusehäufigkeit nachgewiesen werden. Fahrten, die tendenziell eher mit großer Hektik einhergehen und durch Zeitknappheit gekennzeichnet sind, führen zu schlechterer Sicherung (Abbildung 36) ( $p=0,016)$. Das fält insbesondere für Fahrten zur Schule bzw. zum Kindergarten auf, genauso wie für Fahrten im Zusammenhang mit Erledigungen oder auf der Heimfahrt. Bei Situationen, die eher durch Ruhe gekennzeichnet sind, wie z.B. Fahrten in den Urlaub oder Ausflüge, sinkt die Misusehäufigkeit deutlich. 


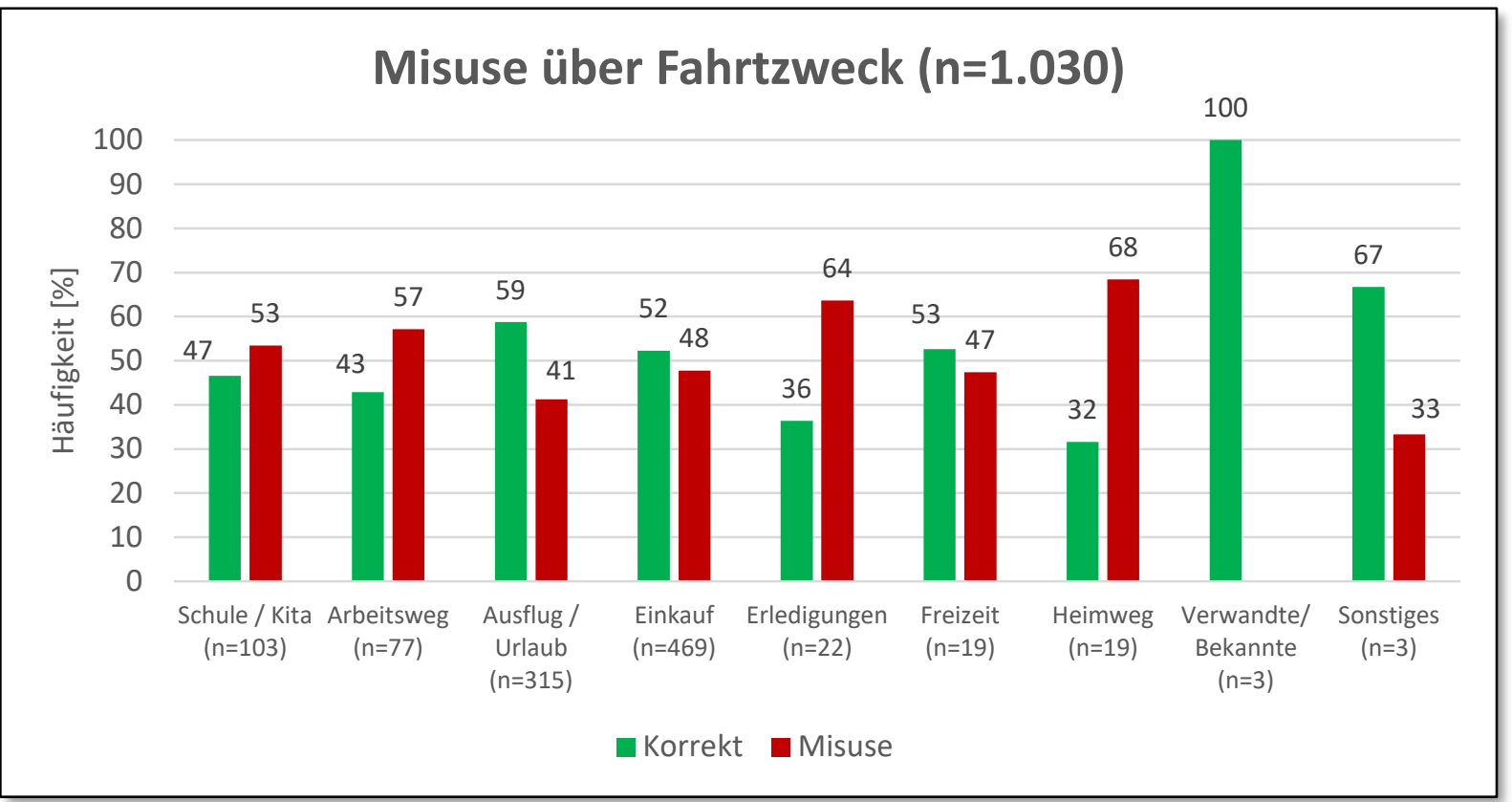

Abbildung 36: Misuse über Fahrtzweck

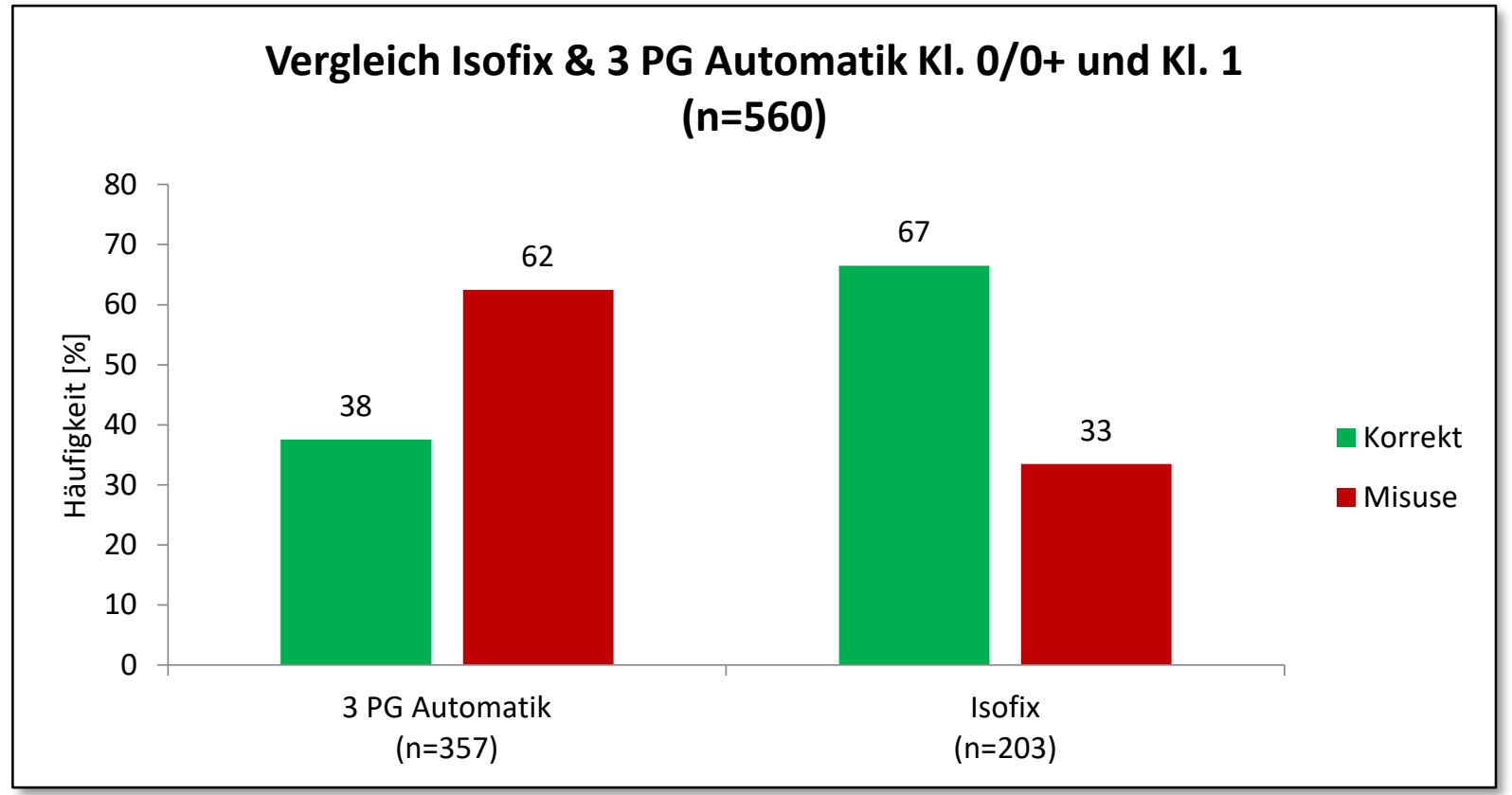

Abbildung 37: Vergleich ISOFIX \& 3PG Automatik in KI.0/0+ und KI.1

Die Zahl der KSS, die mittels ISOFIX im Fahrzeug gesichert sind, ist im Vergleich zu früheren Studien deutlich gestiegen (s. Abbildung 27). Dieser Trend hat ganz unmittelbare 
Auswirkung auf die Häufigkeit von Misuse insgesamt. Wie in Abbildung 37 zu sehen ist, tritt im Zusammenhang mit der Nutzung von ISOFIX hochsignifikant weniger Misuse auf als bei der Sicherung des Kindersitzes mittels Dreipunktgurt $(p=0,000)$. Hier zeigt sich direkt der positive Nutzen eines technischen Standards, der in den letzten Jahren in Fahrzeugen und bei KSS vermehrt vorzufinden ist.

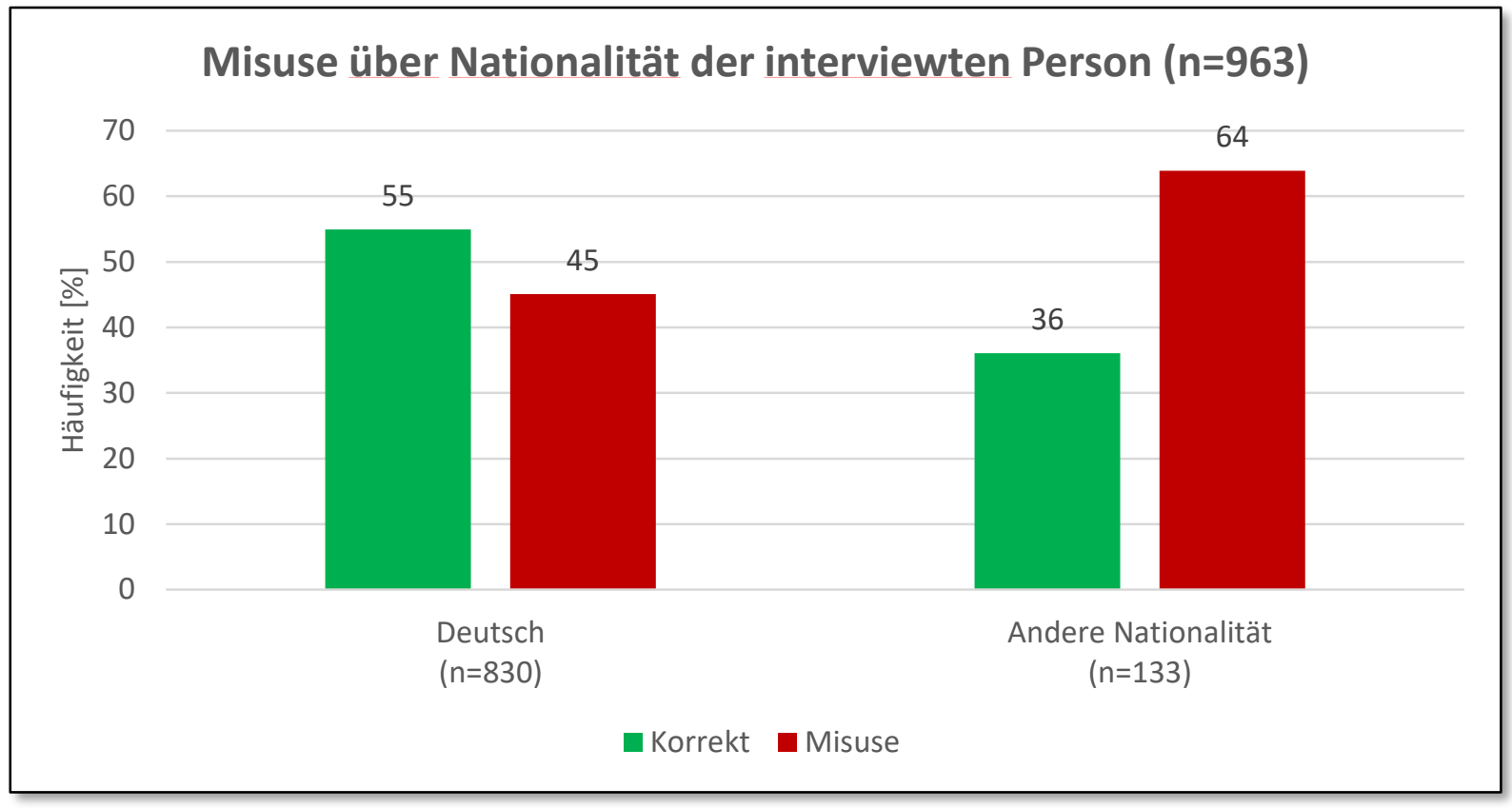

Abbildung 38: Misusequote nach Nationalität der interviewten Person

Ein ebenfalls hochsignifikanter Zusammenhang konnte zwischen der Misusequote und der Staatsangehörigkeit der interviewten (in der Regel der sichernden) Person festgestellt werden. Wie ebenfalls in früheren Studien gezeigt [Fastenmeier, 2006], liegt die Misusequote bei Menschen nicht deutscher Nationalität deutlich höher als bei deutschen Staatsangehörigen $(p=0,000)$. Insbesondere auffällig sind in dieser Hinsicht Menschen aus dem südosteuropäischen Raum, aus der Türkei und aus arabischen Staaten. In den Befragungen hat sich immer wieder gezeigt, dass der beschriebene Effekt insbesondere kulturelle Ursachen hat. Umso nötiger ist es also, für die Menschen, die den genannten Staaten zuzurechnen sind, geeignete Anspracheformen für das Thema der Sicherung von Kindern in Fahrzeugen weiter zu entwickeln und entsprechend umzusetzen (s. auch Kapitel 4).

Die Analyse der Daten hat weiterhin gezeigt, dass die Wahrscheinlichkeit für Misuse signifikant vom Alter der sichernden Person abhängt (Abbildung 39). Als besonders kritisch fällt dabei die Gruppe der unter 30jährigen auf $(p=0,03)$. Das ist die Gruppe, die als junge 
Eltern bezeichnet werden, also jene, deren Kinder in der Regel besonders jung sind. In dieser Gruppe tritt vergleichsweise viel Misuse auf und dieser ist mit einem Anteil von fast $68 \%$ meist schwerer Misuse. Hier zeigt sich einmal mehr, dass Aufklärungsarbeit hinsichtlich der richtigen Benutzung von Kindersitzen insbesondere bei jungen Eltern besonders wichtig ist. Eine weitere auffällige Gruppe mit erhöhtem Misuse ist die der 40-59jährigen Sicherungsakteure.

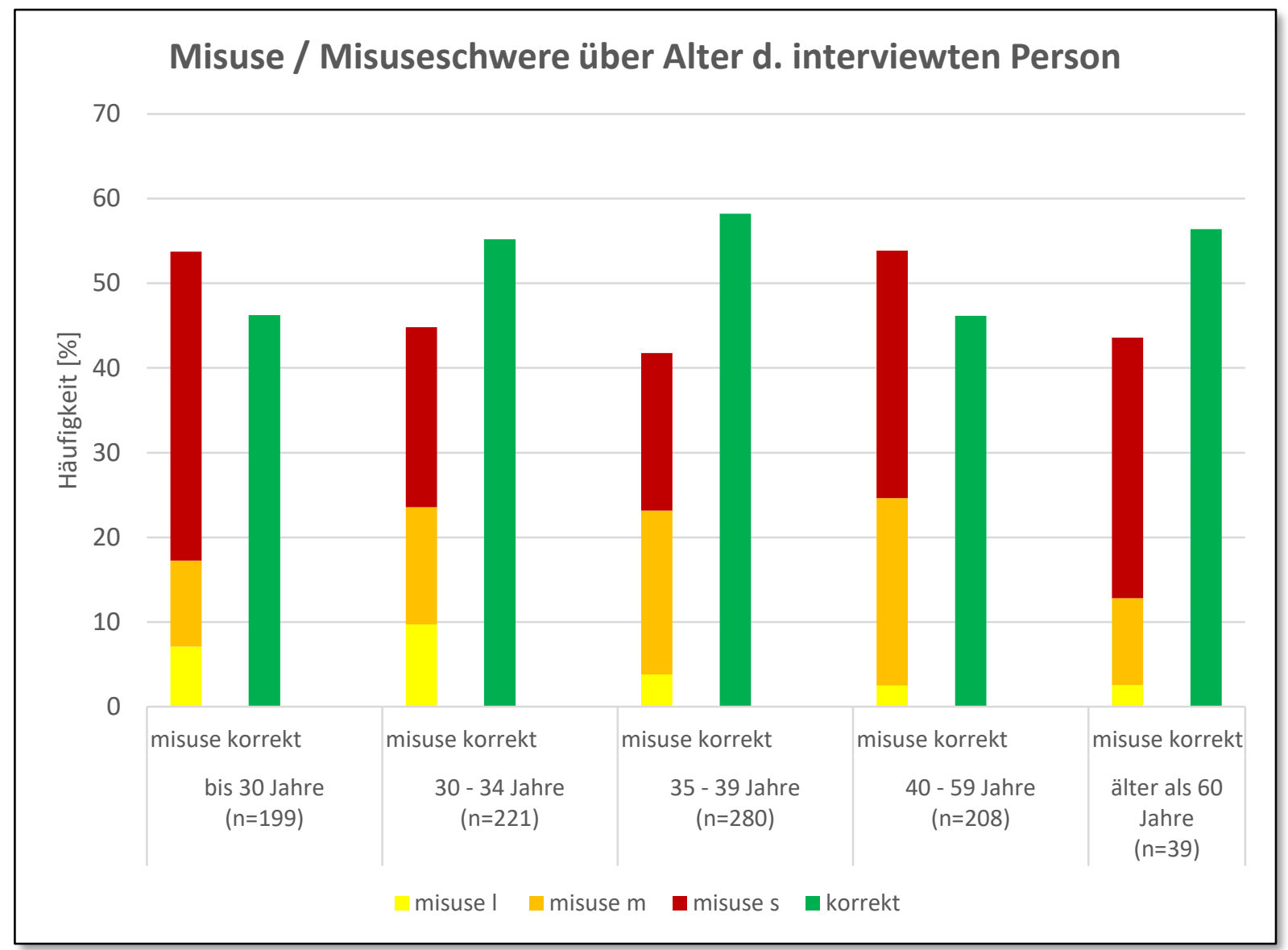

Abbildung 39: Misuse / Misusequote über Alter der interviewten Person

\section{Installation KSS}

Im Folgenden werden Installationsfehler näher untersucht. Als Installationsfehler werden Fehler beschrieben, die beim Einbau des Kindersitzes ins Fahrzeug auftreten. Diese Fehler sind also völlig unabhängig vom Kind, welches den Kindersitz nutzt. 


\begin{tabular}{|l|c|}
\hline \multicolumn{1}{|c|}{ Installationsfehler KI. 0/0+ } & Häufigkeit [\%] \\
\hline Gurtlose im Fahrzeuggurt & 24,8 \\
\hline Führungshilfe nicht eingelegt & 19,3 \\
\hline Becken- und Schultergurt vertauscht & 16,6 \\
\hline Tragegriffposition nicht korrekt & 9,7 \\
\hline Fahrzeuggurt verdreht & 7,6 \\
\hline Sonstige Fehler & 22,1 \\
\hline
\end{tabular}

Tabelle 2: Top 5 Installationsfehler Klasse 0/0+ $(n=145)$

Bei den Installationsfehlern bei Klasse 0/0+-Sitzen dominieren Fehler, die im Zusammenhang mit dem Fahrzeuggurt stehen. Das sind insbesondere die Gurtlose, die Nichtbenutzung der Führungshilfen für den Gurt und das Vertauschen von Becken- und Schultergurt (Tabelle 2). Je nach Ausprägung der Fehler werden alle drei als schwerer Misuse codiert. Insbesondere das Vertauschen von Schulter- und Beckengurt bedeutet in der Realität, dass Kinder und Kindersitz de facto nicht im Fahrzeug gesichert sind. Im Ergebnis haben die drei genannten Misusearten zwei wichtige Gemeinsamkeiten: Sie stellen ein erhebliches Verletzungsrisiko für den kindlichen Insassen dar und sie lassen sich wirkungsvoll durch die Nutzung von ISOFIX verhindern.

Als besonders gravierender Fehler bei Klasse 0/0+-Sitzen gilt nach wie vor die unterlassene Deaktivierung des Beifahrerairbags, wenn dort eine Babyschale installiert ist. Obwohl darauf durch mehrere Warnhinweise im Fahrzeuginnenraum hingewiesen wird und dieser Punkt ein eigener Tatbestand im Bußgeldkatalog ist, war der Airbag in fast $15 \%$ der Fälle, in denen eine Babyschale auf dem Beifahrersitz gesichert war, der Airbag nicht deaktiviert. Das ist umso bemerkenswerter, da in modernen Fahrzeugen das Deaktivieren in der Regel sehr einfach ist. Unfälle, bei denen die Babyschale durch einen entfalteten Airbag getroffen wird, können mit erheblichen Verletzungen für das Kind einhergehen. Technische Lösungen, die diese Fehlerbenutzung verhindern können, gibt es bereits seit einigen Jahren (s. Kapitel 1.3.3). Aufgrund mangelnder Standardisierung haben sich diese aber nicht durchgesetzt.

Mit 16,5\% ebenfalls noch sehr häufig tritt der Fehler des vertauschten Schulter- und Beckengurts auf. Dabei wird der Beckengurtabschnitt hinter der Babyschale geführt und der Schultergurt entsprechend vorn über das Kind. Im Ergebnis ist eine so befestigte 
Babyschale nicht im Fahrzeug gesichert und kann sich ungehindert nach vorn verlagern. Zur Verdeutlichung der Folgen dieser Fehlbenutzung wurden im Rahmen dieses Forschungsprojekts zwei Schlittenversuche durchgeführt, die die Folgen richtiger und falscher Gurtführung eindrücklich demonstrieren (siehe Kapitel 6.6).

\begin{tabular}{|l|c|}
\hline \multicolumn{1}{|c|}{ Installationsfehler KI. 1+ } & Häufigkeit [\%] \\
\hline Gurtlose im Fahrzeuggurt & 49,3 \\
\hline Fahrzeuggurt verdreht & 12,9 \\
\hline Führungshilfe nicht eingelegt & 10,2 \\
\hline $\begin{array}{l}\text { Gurtklemme nicht / falsch } \\
\text { verwendet }\end{array}$ & 4,4 \\
\hline $\begin{array}{l}\text { Isofix ohne Antirotationseinrichtung } \\
\text { Sonstige Fehler }\end{array}$ & 4,0 \\
\hline
\end{tabular}

Tabelle 3: Top 5 Installationsfehler Klasse $1(n=225)$

Für Installationsfehler bei Klasse 1-Sitzen ergibt sich ein sehr ähnliches Bild. Auch in dieser Gruppe treten vor allem Probleme bei der Interaktion mit dem Fahrzeuggurt auf (Tabelle 3). Dominant ist dabei die Gurtlose im Fahrzeuggurt, die in knapp der Hälfte aller untersuchten Klasse 1-Sitze zu finden war. Die Gurtlose wird je nach Ausprägung mit unterschiedlichem Schweregrad bewertet. Bei den hier untersuchten Fällen trat vor allem schwere Gurtlose auf (63\% schwere Gurtlose, $26 \%$ mittlere Gurte, $11 \%$ leichte Gurtlose). Das setzt die Schutzwirkung eines KSS erheblich herab und kann im Ergebnis zu schweren Verletzungen des Kindes führen.

Auch hier gilt, dass dieser und auch die weiteren drei aufgeführten Fehler in Tabelle 3 durch die Verwendung von ISOFIX verlässlich verhindert werden würden. Bemerkenswert ist, dass der fünfthäufigste aufgetretene Fehler im Zusammenhang mit der Installation von Klasse 1Sitzen in Verbindung mit ISOFIX steht. In vier Prozent aller Klasse 1-Sitze wurde die sogenannte Antirotationseinrichtung, also der Stützfuß bzw. der TopTether nicht benutzt. Dieser Fehler wird im Codebook als „schwer“ codiert. Daraus ergeben sich zwei wesentliche Erkenntnisse. Zum einen spricht das für eine erfreulich hohe Verbreitung für ISOFIX-Sitze, wenn diese bereits in der Fehlerstatistik der Gesamtheit aller Klasse 1-Sitze auftauchen. Zum anderen zeigt sich einmal mehr, dass trotz der insgesamt recht problemlosen Nutzung 
von ISOFIX die Verwendung dieser Antirotationseinrichtungen den Nutzern Probleme bereitet. Hier scheint weiterhin Verbesserungsbedarf zu bestehen.

\begin{tabular}{|l|c|}
\hline \multicolumn{1}{|c|}{ Installationsfehler KI. 2/3+ } & Häufigkeit [\%] \\
\hline Decke (o.Ä.) unter KSS & 11,4 \\
\hline Eigene Konstruktion & 11,4 \\
\hline $\begin{array}{l}\text { Interaktionsproblem mit Kopfstütze } \\
\text { (vom Fahrzeug) }\end{array}$ & 11,4 \\
\hline Unzulässige Einbauposition & 5,7 \\
\hline KSS beschädigt & 5,7 \\
\hline $\begin{array}{l}\text { Isofix gurtschlossseitig nicht } \\
\text { eingehakt }\end{array}$ & 5,7 \\
\hline \begin{tabular}{l} 
Sonstige Fehler \\
\hline
\end{tabular} & 48,6 \\
\hline
\end{tabular}

Tabelle 4: Top 6 Installationsfehler Klasse 2/3 (n=35)

Für Installationsfehler bei den 2/3-Sitzen ergibt sich ein weniger eindeutiges Bild (Tabelle 4). Viele verschiedene Fehler konnten beobachtet werden, die jeweils nur in geringer Häufigkeit aufgetreten sind. Generell ist aber bei der Installation eines solchen Sitzes ohnehin wenig zu beachten, da der Sitz selbst gemeinsam mit dem Kind im Fahrzeug gesichert wird. Im Wesentlichen treten für diese Sitzklasse daher Sicherungsfehler auf.

\section{Sicherung Kind}

Unter der Sicherung des Kindes versteht man das Sichern des Kindes im Kindersitz. Das geschieht entweder durch ein eigenes Gurtsystem des KSS, durch einen Fangkörper, der zum KSS gehört oder direkt durch den Fahrzeuggurt, der dann KSS und Kind gemeinsam sichert. Im Folgenden werden Fehler beschrieben, die im Zusammenhang mit diesem Sicherungsvorgang beobachtet wurden.

Bei KSS der Klasse 0/0+ trat ganz überwiegend die Gurtlose im sitzintegrierten Gurtsystem auf. Dieser Fehler machte über $90 \%$ aller Sicherungsfehler in Klasse 0/0+-Sitzen aus. Je nach Ausprägung dieses Fehlers können die Auswirkungen im Falle eines Unfalls 
unterschiedlich sein. Ist die Gurtlose derart groß, dass die Schultergurte über die Schultern rutschen können und das Baby dadurch unter dem Gurt hindurchrutschen kann, ist es im schlimmsten Fall nicht gesichert und verlässt das KSS ungehindert. Andere festgestellte Fehler traten in nur sehr geringer Häufigkeit auf.

Das Problem der Gurtlose war auch bei Klasse 1-Sitzen dominant und machte runde $90 \%$ aller Sicherungsfehler aus. Auch hier gilt, dass dieser Fehler dann besonders schwerwiegend ist, wenn der Schultergurt auf Höhe der Oberarme des Kindes verläuft und somit keine Rückhaltung des Oberkörpers erfolgt. Im Fall eines Frontalaufpralls kommt es zum sogenannten Klappmessereffekt. Das bedeutet, dass der Oberkörper nach vorn klappt und das Kind allein durch den unteren Gurtabschnitt des KSS-Gurts zurückgehalten wird. Entsprechend groß sind die Kräfte, die in den Abdominalbereich eingeleitet werden, was zu gravierenden Verletzungen führen kann. Hinzu kommt eine erhebliche Vorverlagerung des Oberkörpers, die das Risiko eines Kopfaufpralls erheblich erhöht. Eine hilfreiche Lösung für dieses Problem stellt der Chestclip dar. Dieser verbindet die beiden Schultergurtpartien und verhindert so auch bei größerer Gurtlose, dass diese über die Schultern rutschen können. 


\begin{tabular}{|l|c|}
\hline \multicolumn{1}{|c|}{ Sicherungsfehler KI. 2/3+ } & Häufigkeit [\%] \\
\hline Gurtlose des Beckengurtes & 44,8 \\
\hline Fahrzeuggurt verdreht & 11,5 \\
\hline Gurtlose des Schultergurtes & 9,4 \\
\hline $\begin{array}{l}\text { Führungshilfe Beckengurt } \\
\text { gurtschlossseitig nicht eingelegt }\end{array}$ & 9,4 \\
\hline $\begin{array}{l}\text { Führungshilfe Schultergurt } \\
\text { gurtankerseitig nicht eingelegt }\end{array}$ & 8,6 \\
\hline $\begin{array}{l}\text { Führungshilfe Beckengurt } \\
\text { gurtankerseitig nicht eingelegt }\end{array}$ & 4,7 \\
\hline $\begin{array}{l}\text { Schultergurt unter dem Arm } \\
\text { geklemmt }\end{array}$ & 1,6 \\
\hline $\begin{array}{l}\text { Gurtverlauf mit Halskontakt } \\
\text { gurtschlossseitig eingelegt }\end{array}$ & 2,9 \\
\hline $\begin{array}{l}\text { Kopfstütze (KSS) zu hoch } \\
\text { eingestellt }\end{array}$ & 2.1 \\
\hline
\end{tabular}

Tabelle 5: Sicherungsfehler in Klasse 2/3 nach Häufigkeit absteigend

Auch bei der Sicherung des Kindes im Klasse 2/3-Sitz trat als Fehler vornehmlich Gurtlose im Beckengurt auf (Tabelle 5). Darüber hinaus wurde eine Vielzahl von Fehlern festgestellt, die die Gurtführung des Fahrzeuggurts betreffen. Manche davon werden gemäß Codebook als leichte Fehler eingestuft wie zum Beispiel die zu hoch eingestellte Kopfstütze des KSS. Andere Misuseformen hingegen können schwerwiegende Folgen haben und werden entsprechend als "schwer“ kategorisiert. Dazu zählt beispielsweise der Schultergurt, der unter der Schulter des Kindes verläuft und somit den Oberkörper des Kindes nicht mehr zurückhalten kann. Ähnlich wie beim Klasse 1-Sitz beschrieben, kann auch das zu ernsthaften Verletzungen im Abdomen führen. 


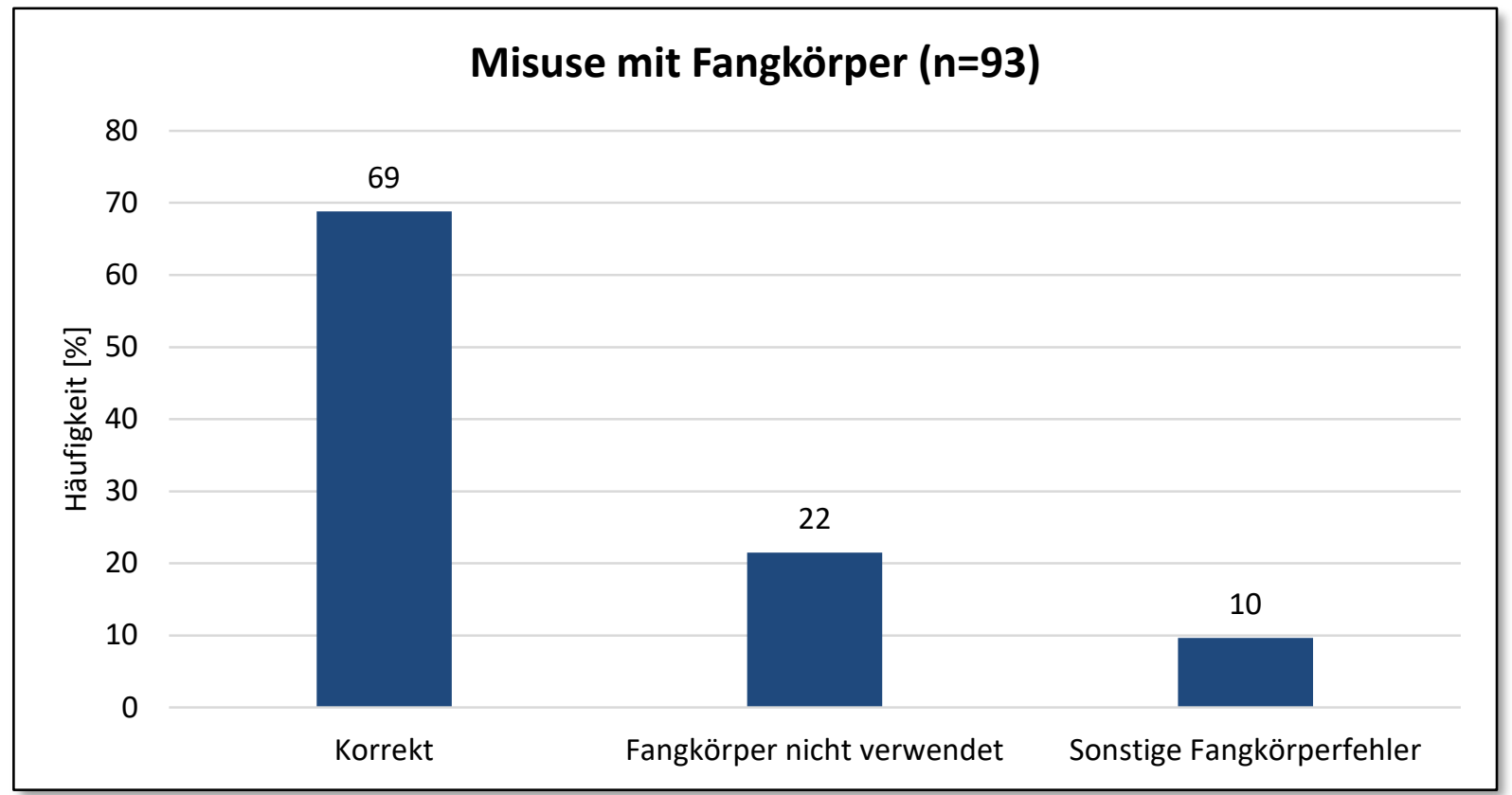

Abbildung 40: Misuse mit Fangkörper

Mit stärker Verbreitung der Fangkörpersitze sind für dieses Modell spezifische Fehler häufiger vorzufinden. Das betrifft insbesondere die Nichtverwendung des Fangkörpers (Abbildung 40). Wird der Fangkörper in einem Klasse 1-Sitz weggelassen, so wird das Kind direkt über den Fahrzeuggurt gesichert. Damit entspricht das KSS dann einem Klasse 2/3Sitz, für den das Kind zu klein ist. Eine sichere und dem Kind angepasste Rückhaltung ist nicht möglich.

\section{Sonstige Fehler}

Unabhängig von der richtigen Installation des KSS im Fahrzeug und der korrekten Sicherung des Kindes im Kindersitz können generelle Passungsprobleme auftreten. Das ist dann der Fall, wenn das Kind hinsichtlich seiner Größe oder seines Gewichts nicht zum Kindersitz passt. In Bezug auf die Gesamtheit aller erhobenen Fälle treten diese Passungsfehler erfreulich selten auf (Abbildung 41). Dennoch kann der aus früheren Studien bekannte Trend festgestellt werden, dass der Wechsel in die nächst höhere Klasse bisweilen zu früh erfolgt, das Kind also für den genutzten Kindersitz zu leicht oder zu klein ist. Dieser Effekt ist insbesondere für den Wechsel in Klasse 2/3-Sitze festzustellen. 


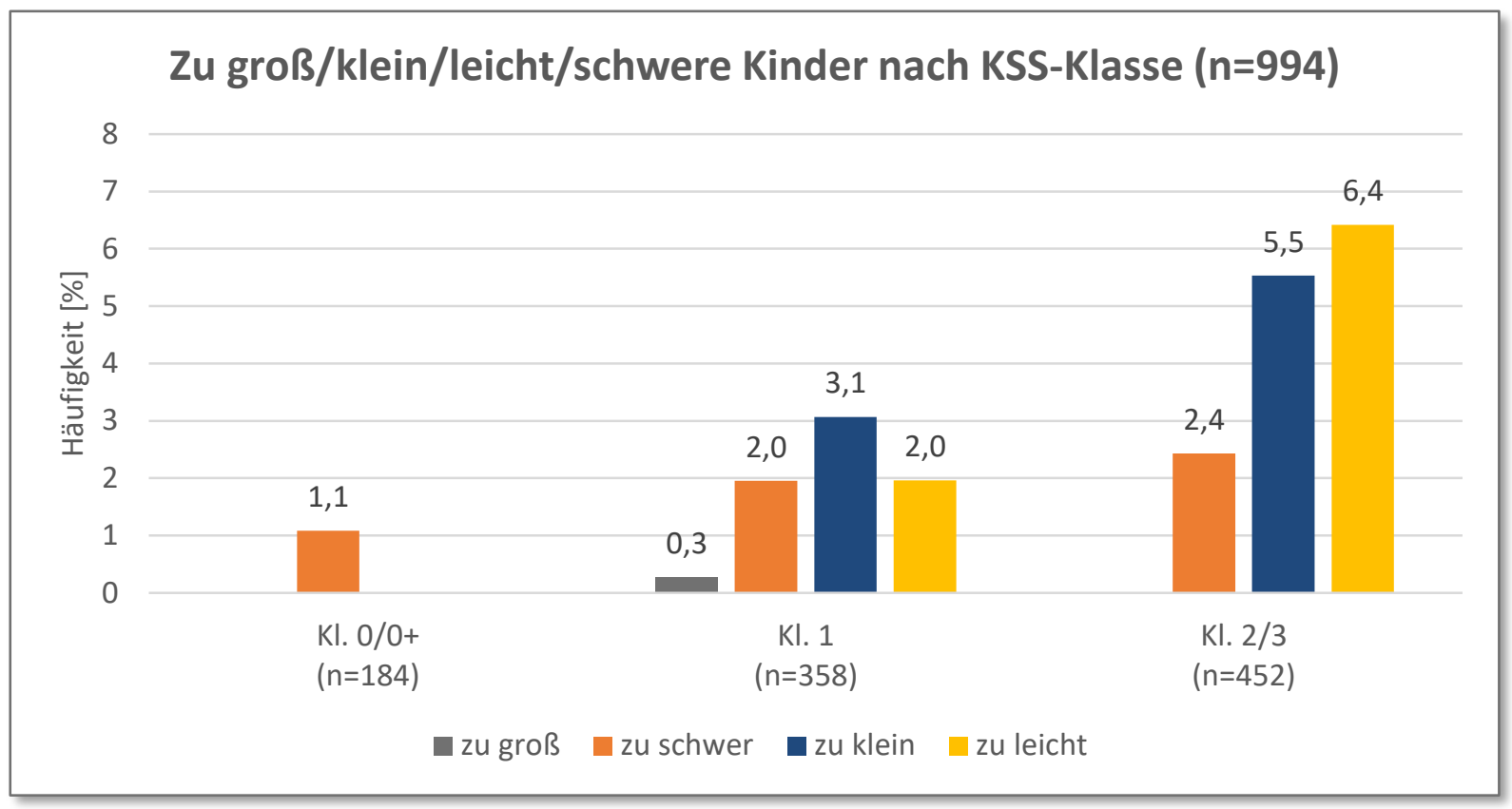

Abbildung 41: Kind passt nicht zum KSS

\section{Einschätzungen der Sicherungsakteure}

Am Ende der Vor-Ort-Überprüfungen der KSS folgte noch eine Reihe von Fragen an die Sicherungsakteure. Auf die Frage "Glauben Sie, dass alles in Ordnung/richtig gesichert war?" antworteten über $80 \%$ der Befragten mit ja. Auffallend ist allerdings, dass über $70 \%$ derjenigen, bei denen Misuse festgestellt worden war, auch der Überzeugung waren, alles richtig gemacht zu haben (vgl. Abbildung 42). 


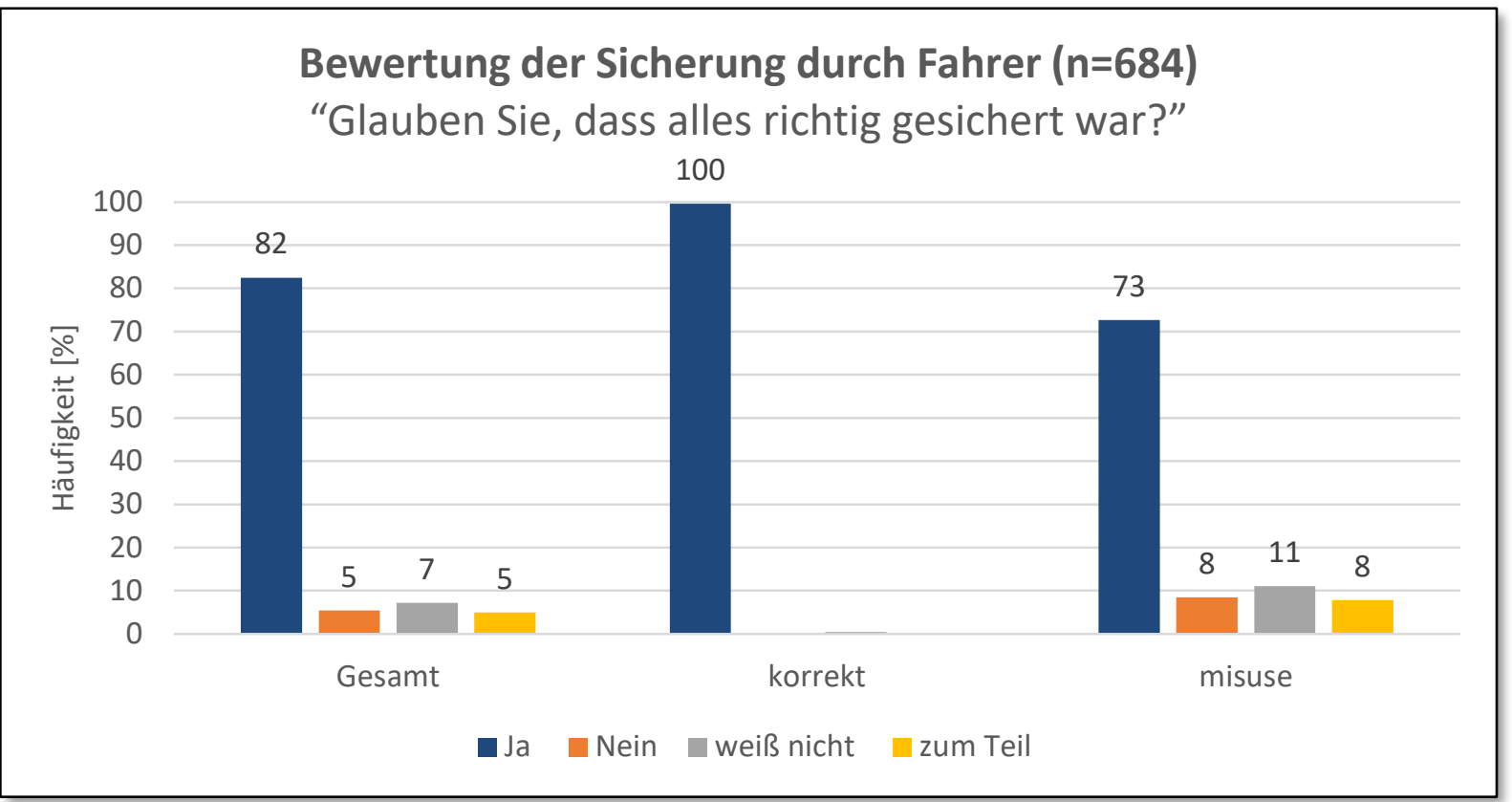

Abbildung 42: Bewertung der Sicherung durch den Fahrer

Nach dem festgestellten Misuse durch die Beobachter wurden die Probanden gefragt, wie sie die dadurch bewirkte Sicherheitseinbuße bewerten: $26 \%$ glaubten, damit wäre „keine Einbuße" verbunden, $13 \%$ stuften sie als "gering" ein, als „erheblich“ und "stark“ klassifizierten $9 \%$ bzw. $14 \%$ ihre Sicherung. Immerhin $37 \%$ konnten sich zu keiner Beurteilung durchringen und antworteten „weiß nicht“. Betrachtet man diese Einschätzungen nach den einzelnen KSS-Klassen, so ergeben sich weitgehend kongruente Antworten: Wie in Abbildung 43 dargestellt, ist ein erheblicher Teil der Befragten der Auffassung, eine fehlerhafte Handhabung der Kindersitze hätte keinerlei Folgen für die Schutzwirkung der Systeme: der Anteil dieser Teilgruppe reicht von knapp $20 \%$ bei schwerem Misuse über $30 \%$ bei mittlerem Misuse bis $40 \%$ bei leichtem Misuse. Nur ein geringer Teil äußerte eine realistische Einschätzung der damit verbundenen Risiken. Auffallend sind wiederum die Antworten mit „weiß nicht“: Sie umfassen in der Regel den größten Anteil der Antworten und reichen von knapp $40 \%$ bei leichten Misuse bis zu knapp $45 \%$ bei mittlerem Misuse. Bei schwerem Misuse übertrifft „weiß nicht“ mit knapp $35 \%$ bei weitem alle anderen Antwortmöglichkeiten. 


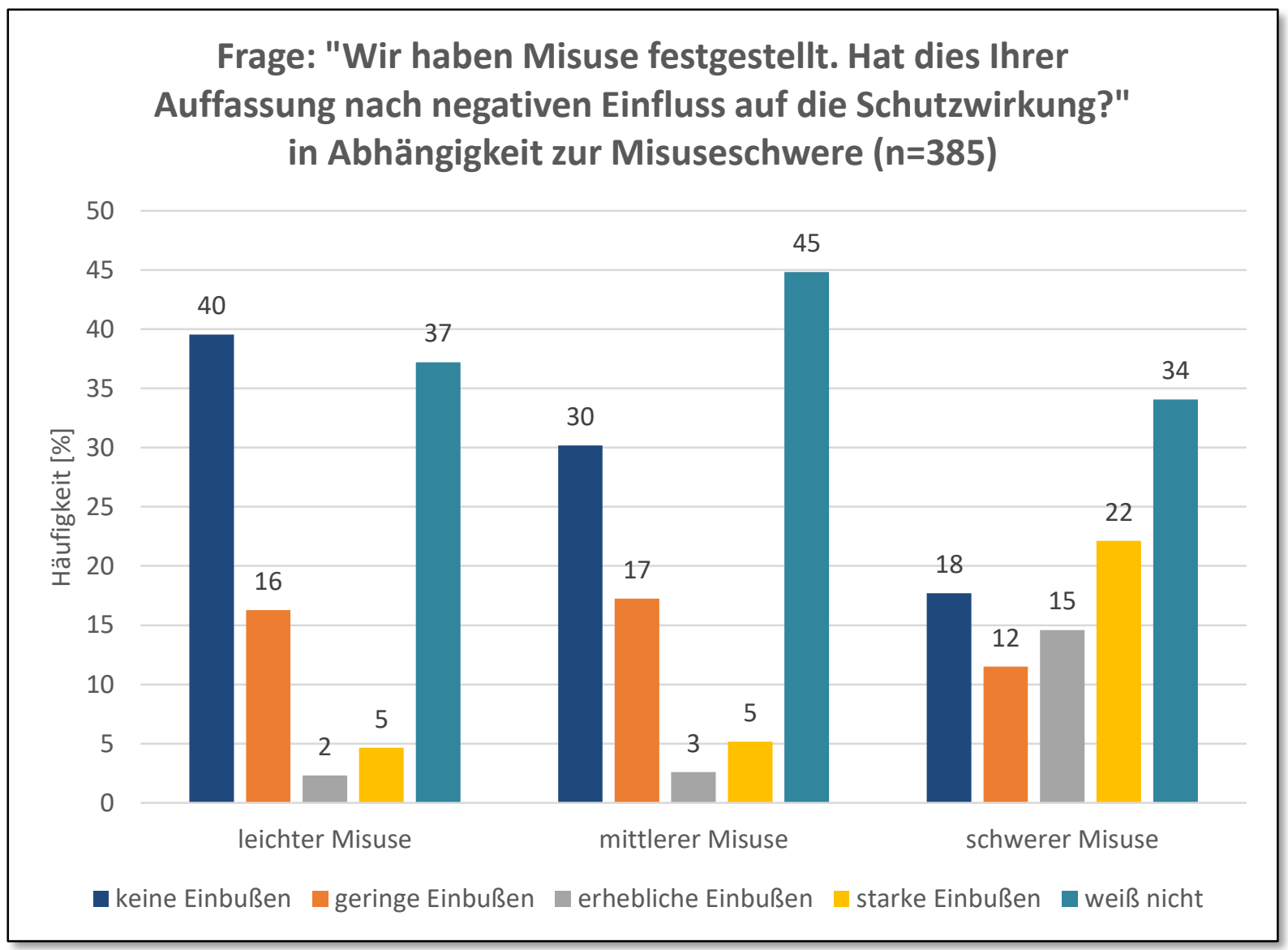

Abbildung 43: Antwort auf die Frage welchen Einfluss auf die Schutzwirkung der festgestellte Misuse hat.

Als Antwort auf die Frage nach den Ursachen für den festgestellten Misuse gaben die Befragten am häufigsten „unbewusste Nachlässigkeit“ an (vgl. Tabelle 6). Das bestätigt wiederum bekannte Ergebnisse. Weitere quantitativ bedeutende Ursachen waren Wissensdefizite in Bezug auf die korrekte Handhabung der Schutzsysteme, mangelnde / fehlerhafte Informationen zur korrekten Handhabung der Schutzsysteme, kurze Fahrtstrecke, Zeitdruck, der Wunsch nach Komfortverbesserung für das Kind, kleidungsbedingter Misuse, das Kind sichert sich selbst sowie „bewusste Nachlässigkeit“. Punkte wie technische Probleme, umständliches Einbauen etc. spielten eine vergleichsweise untergeordnete Rolle. 


\begin{tabular}{|l|c|}
\hline \multicolumn{1}{|c|}{ Ursachen für Misuse } & Häufigkeit [\%] \\
\hline unbewusste Nachlässigkeit & 32,9 \\
\hline Unkenntnis & 10,1 \\
\hline kurze Strecke & 9,1 \\
\hline mangelnde / fehlerhafte Information & 6,2 \\
\hline Kind sichert sich selbst & 6,7 \\
\hline Komfortverbesserung fürs Kind & 5,9 \\
\hline Kurze Strecke & 5.5 \\
\hline Zeitdruck & 5,3 \\
\hline Bewusste Nachlässigkeit & 2,7 \\
\hline technisch bedingt & 3,3 \\
\hline Kleidungsbedingt (dick/dünn) & 3,3 \\
\hline Widerstand des Kindes & 3,2 \\
\hline umständliches Einbauen/Sichern & \\
\hline Zweit-/Fremdauto, Fahrzeugwechsel & \\
\hline Mehrere Kinder & \\
\hline Sitzwechsel der Kinder & \\
\hline
\end{tabular}

Tabelle 6: Ursachen für Misuse (Mehrfachantworten waren möglich) n=321

Eine abschließende Frage richtete sich auf die von den Befragten eingeschätzte generelle Schutzwirkung von KSS: Interessant erscheint, dass der Löwenanteil der Interviewpartner (91\%) die Schutzwirkung von KSS mit sehr gut oder gut bewertete. Lediglich 8,7 \% kamen 
zur Einschätzung befriedigend bzw. ausreichend und nur eine kleine Minderheit war gar nicht überzeugt. Die Beurteilungen zu den einzelnen KSS-Klassen unterscheiden sich dabei nicht. Kontrastiert man allerdings diese Einschätzung der Befragten mit dem durch die Beobachter festgestellten Misuse, dann fällt auf: Insbesondere bei denjenigen, die eine stark positive Einschätzung der Sicherheitswirkung von KSS haben, wurde signifikant häufiger Misuse festgestellt $(p<0,001)$.

Zusammenfassend lässt sich hier festhalten: Der große Anteil, den der Faktor unbewusste Nachlässigkeit bei den Begründungen für Misuse besitzt, weist möglicherweise darauf hin, dass die KSS systemübergreifend und konstruktionsseitig subjektive Unaufmerksamkeiten ermöglichen oder sogar fördern.

Die Ergebnisse sind auch ein gewichtiger Indikator dafür, dass Wissensdefizite sowohl beim Einbau als auch beim Sichern schwere Sicherheitseinbußen der verwendeten Schutzsysteme nach sich ziehen.

\subsection{Telefonische Nachbefragung}

Der zweite Teil des Erhebungskonzepts sah vor, mit einer Teilstichprobe der im Feld befragten Nutzer ein weiteres Interview durchzuführen, in denen die im Kontext der (fehlerhaften) Kindersicherung festgestellten Probleme in einem persönlichen Telefonat vertieft bzw. abgeklärt werden sollten. Diese Interviews sollten insbesondere Aufschluss über die Begründungen für ggfs. festgestellten Misuse und somit auch - in Ergänzung bekannter Erkenntnisse - zur Ursachenklärung von Misuse beitragen.

\section{Fragebogen}

Der Erfassung von Kontextbedingungen, Handlungsmotiven und handlungsrelevanten Wissenselementen zu KSS diente die telefonische Nachbefragung. Der dazu verwendete Fragebogen lehnte sich an das schon bewährte Instrument von Fastenmeier \& Lehnig (2006) an. Die Erfahrungen in den Pretests sowie an den ersten Erhebungstagen führtenwie schon erwartet - dazu, den Beobachtungs- und Befragungsteil zu trennen (zu großer Zeitaufwand mit ca. 20-25 Minuten in den Augen der Probanden). Die Probanden wurden also nach ihrer Bereitschaft gefragt, an einer telefonischen Nachbefragung teilzunehmen, die zeitnah innerhalb weniger Tage stattfinden sollte.

Der Fragebogen für die Nachbefragung umfasste ebenso wie der Beobachtungsbogen vier Teile und zwar:

- Fragen zum Erwerb des Kindersitzes 
- Fragen zum Einbau des Kindersitzes (gilt in der Regel für KSS, bei denen Einbau und Sichern zwei getrennte Vorgänge sind)

- Fragen zum Sichern im Kindersitz

- allgemeine Fragen zum Sichern im Kindersitz (handlungsrelevante Wissenselemente).

\section{Ergebnisse der Nachbefragungen}

Bedauerlicherweise war die Bereitschaft der Probanden, zusätzlich an der (telefonischen) Nachbefragung teilzunehmen, äußerst gering. Insgesamt haben $n=107$ Probanden an der Nachbefragung teilgenommen. Die Probanden lehnten eine Teilnahme entweder direkt vor Ort ab oder sie gaben z.T. Telefonnummern heraus, die nicht oder nur über AB erreichbar waren, so dass nach mehreren vergeblichen Versuchen abgebrochen werden musste. Über die Gründe dieser Ablehnung kann nur spekuliert werden, vor Ort waren die meisten Probanden zugänglich, eine Reihe von Probanden reagierte allerdings - nachdem Misuse festgestellt worden war - unfreundlich auf die Interviewer.

Misuse bei den Nachbefragungen.- Misuse und korrekte Benutzung halten sich in der Nachbefragung in etwa die Waage, womit die Misusequote in den Nachbefragungen einen geringfügig höheren Anteil ausweist als in den Vor-Ort-Beobachtungen.

Status, Alter und Herkunft der KSS.- Etwas mehr als $70 \%$ der untersuchten KSS war neu, knapp $16 \%$ gebraucht erworben und etwas mehr als $9 \%$ bekamen die Nutzer als Geschenk; der Gebrauch geliehener KSS kam selten vor (vgl. Tabelle 7).

\begin{tabular}{|l|c|}
\hline \multicolumn{1}{|c|}{ KSS: Wie erworben? } & Häufigkeit [\%] \\
\hline neu & 71,0 \\
\hline gebraucht & 15,9 \\
\hline geschenkt bekommen & 9,3 \\
\hline geliehen & 3,7 \\
\hline
\end{tabular}

Tabelle 7: Status der KSS ( $n=107)$

Das Alter der verwendeten Schutzsysteme reichte von 3 Monaten bis 14 Jahre. Der Großteil der Kindersitze war allerdings höchstens 2-4 Jahre alt, etwa ein Drittel jünger als 2 Jahre (vgl. Tabelle 8). 


\begin{tabular}{|l|c|}
\hline \multicolumn{1}{|c|}{ KSS: Wann erworben? } & Häufigkeit [\%] \\
\hline vor 2013 & 14,7 \\
\hline 2013 bis 2015 & 53,7 \\
\hline nach 2015 & 31,6 \\
\hline
\end{tabular}

Tabelle 8: Alter der KSS ( $n=107)$

Die Kindersitze wurden am häufigsten in Fachgeschäften erworben (44\%), gefolgt von Käufen im Internet (31\%). Relativ häufig wurden sie allerdings auch entweder gebraucht von Privatpersonen erstanden oder sie waren ein Geschenk. Super-/ Baumärkte, Kindersitzbörsen etc. spielten keine Rolle in der untersuchten Stichprobe (vgl. Tabelle 9).

\begin{tabular}{|l|c|}
\hline \multicolumn{1}{|c|}{ Herkunft der KSS } & Häufigkeit [\%] \\
\hline Fachgeschäft & 44,0 \\
\hline Internet & 31,0 \\
\hline privat & 19,0 \\
\hline Super-/Baumarkt & 1,0 \\
\hline Kindersitzbörse & 1,0 \\
\hline andere Herkunft & 4,0 \\
\hline
\end{tabular}

Tabelle 9: Herkunft der KSS ( $\mathrm{n}=107)$

Informationsbeschaffung und maßgebliche Gesichtspunkte beim Erwerb eines KSS.Vor dem Erwerb eines KSS wurden am häufigsten Testberichte in Zeitschriften sowie das Internet zu Rate gezogen; große Bedeutung wurde aber auch Ratschlägen von Verwandten / Bekannten beigemessen. Beratung im Fachgeschäft oder durch ADAC u.ä. spielte demgegenüber eine etwas geringere, aber immer noch nennenswerte Rolle (vgl. Tabelle 10). 


\begin{tabular}{|l|c|}
\hline \multicolumn{1}{|c|}{ Informationsquellen } & Häufigkeit [\%] \\
\hline Testberichte (z.B. in Zeitschriften) & 29,1 \\
\hline Internet & 25,9 \\
\hline Verwandte/Bekannte & 17,9 \\
\hline Beratung im Fachgeschäft & 11,6 \\
\hline Beratung durch ADAC o.ä. & 9,5 \\
\hline Sonstiges & 5,8 \\
\hline
\end{tabular}

Tabelle 10: Informationsquellen vor Erwerb der KSS (n=107); (Mehrfachantworten waren möglich)

Als ausschlaggebende Gesichtspunkte beim Erwerb eines Schutzsystems erwiesen sich insbesondere positive Testergebnisse, gefolgt von Kriterien wie Eignung für das Kind, Hersteller / Marke, einfache Handhabung, Komfort für das Kind sowie der Preis. Etwas seltener wurden Tipps von Verwandten / Bekannten, eine Beratung im Fachgeschäft oder die Entsprechung mit ECE-Normen genannt (vgl. Tabelle 11). Die relativ große Bedeutung von Herstellern / Marken dürfte eng mit der Präferenz der Befragten für Testergebnisse verbunden sein. Insgesamt ist davon auszugehen, dass der Großteil der Schutzsysteme ohne direkte fachliche Beratung erworben worden ist, die allerdings möglicherweise indirekt über Testberichte und Informationen ersetzt wurde. Der Preis spielte bei den Kaufentscheidungen offensichtlich eine eher untergeordnete Rolle. 


\begin{tabular}{|l|c|}
\hline \multicolumn{1}{|c|}{ Aspekte für Erwerb eines KSS } & Häufigkeit [\%] \\
\hline Testergebnisse & 23,4 \\
\hline Eignung für das Kind (Alter, Gewicht) & 10,2 \\
\hline Hersteller/Marke & 9,5 \\
\hline einfache Handhabung & 9,1 \\
\hline Komfort für das Kind & 8,4 \\
\hline Kosten / Preis & 8,0 \\
\hline Tipps von Verwandten/Bekannten & 6,2 \\
\hline Beratung im Fachgeschäft & 3,6 \\
\hline ECE-Norm & 3,2 \\
\hline sonstige Gesichtspunkte & 17,9 \\
\hline
\end{tabular}

Tabelle 11: Gesichtspunkte beim Erwerb der KSS ( $n=107)$; (Mehrfachantworten waren möglich)

Charakteristika beim Einbau von Kindersitzen.- Schutzsysteme, bei denen Einbau und Sicherung zwei getrennte Vorgänge sind, wurden von den Befragten überwiegend selbst eingebaut. Mehrheitlich (57,5 \%) führten diese vor Fahrtantritt eine Kontrolle durch, ob das KSS korrekt eingebaut war. Der Verzicht auf eine solche Kontrolle wurde in der Regel damit begründet, dass sie nicht gewohnheitsmäßig vorgenommen werde.

Die von den Befragten berichteten Installationsprobleme (vgl. Tabelle 12) beziehen sich häufig auf die Fahrzeuggurte: So wurde am häufigsten komplizierte Gurtführung benannt $(17,9 \%)$, aber auch zu kurze Fahrzeuggurte und die Notwendigkeit, die Gurte ständig nachprüfen zu müssen. Häufige Nennungen betrafen zudem den aufwendigen Aus- / Einbau bei Fahrzeugwechsel, mangelnde Kompatibilität zwischen KSS und Fahrzeug sowie die eingeschränkte Zugänglichkeit bei 2-türigen Fahrzeugen. Die Antworten entsprechen somit dem, was aus den früheren Studien bekannt ist [Fastenmeier \& Lehnig, 2006]. 


\begin{tabular}{|l|c|}
\hline \multicolumn{1}{|c|}{ Schwierigkeiten beim Einbau } & Häufigkeit [\%] \\
\hline komplizierte Gurtführung & 17,9 \\
\hline $\begin{array}{l}\text { aufwendiger Aus-/Einbau bei } \\
\text { Fahrzeugwechsel }\end{array}$ & 12,8 \\
\hline $\begin{array}{l}\text { schlechte Passung zwischen Kindersitz } \\
\text { und Fahrzeug }\end{array}$ & 12,8 \\
\hline $\begin{array}{l}\text { schwere Zugänglichkeit bei 2-türigem } \\
\text { Fahrzeug }\end{array}$ & 10,2 \\
\hline Fahrzeuggurte zu kurz & 10,2 \\
\hline schwer verständliche Anleitung & 5,1 \\
\hline $\begin{array}{l}\text { ständige Nachprüfung der Gurte } \\
\text { erforderlich }\end{array}$ & 5,1 \\
\hline weitere Probleme & 25,6 \\
\hline
\end{tabular}

Tabelle 12: Schwierigkeiten beim Einbau $(n=39)$; (Mehrfachantworten waren möglich)

Als Begründungen für Einbauprobleme wurden am häufigsten die mangelnde Eignung des Fahrzeugs für den Sitz, aber auch fehlende Informationen in der Herstelleranleitung, wenig benutzerunfreundliche Kindersitze sowie die eigene Ungeschicklichkeit angeführt. Dazu kommen Platzprobleme bei mehreren Sitzen sowie Einbauprobleme bei Zweit/ Fremdwagen (vgl. Tabelle 13). Auch hier bewegen sich die Antworten analog zu früheren Erkenntnissen.

\begin{tabular}{|l|c|}
\hline \multicolumn{1}{|c|}{ Begründungen für Einbauprobleme } & $\%$ \\
\hline Fahrzeug nicht für Sitz geeignet & 30,6 \\
\hline Sitz benutzerunfreundlich & 8,3 \\
\hline $\begin{array}{l}\text { fehlende in } \\
\text { Herstelleranleitung }\end{array}$ & 8,3 \\
\hline eigene Ungeschicklichkeit & 8,3 \\
\hline Platzprobleme bei mehreren Sitzen & 8,3 \\
\hline $\begin{array}{l}\text { Einbauprobleme wegen Zweit- } \\
\text { /Fremdwagen }\end{array}$ & 5,6 \\
\hline weitere Gründe & 30,6 \\
\hline
\end{tabular}

Tabelle 13: Begründungen für Einbauprobleme ( $n=39)$; (Mehrfachantworten waren möglich) 
Als Hilfestellung beim Einbau nutzten die Befragten in erster Linie die Herstelleranleitung, gefolgt vom einfachen Ausprobieren. Weitere Orientierungshilfen waren der Probeeinbau im Fachgeschäft sowie Tipps von Verwandten / Bekannten.

Charakteristika beim Sichern der Kinder.- Über $60 \%$ der Befragten erklärten, keine Schwierigkeiten beim Sicherungsvorgang zu haben, etwa ein Viertel war dabei schon „manchmal“ und knapp jeder Zehnte schon „öfter“ mit Problemen konfrontiert. Als häufigste Schwierigkeit beim Sichern führten die Befragten „Kind wehrt sich bei / gegen Sicherung“ (vgl. Tabelle 14). Durch Kinder hervorgerufene Schwierigkeiten (neben „Kind wehrt sich bei / gegen Sicherung“ betrifft das „Kind manipuliert unterwegs die Sicherung“, „Kind öffnet Sicherung während der Fahrt“) umfassten etwa die Hälfte aller angegebenen Probleme im Kontext der Sicherung. Dieser Anteil liegt höher als in der erwähnten Studie von Fastenmeier \& Lehnig (2006). Dieser Befund ist allerdings angesichts der geringen Fallzahlen mit Vorsicht zu interpretieren. Häufiger genannt wurden zudem Schwierigkeiten mit der Handhabung der Gurte („Gurte verdrehen sich“, „komplizierte Gurtführung“, „Gurtschlossposition verlagert sich“ und „zu lockere Gurte bei Kleidungswechsel“). Als Ursachen für derartige Probleme wurden von den Befragten dementsprechend „Widerstand des Kindes“ sowie "Benutzerunfreundlichkeit des Kindersitzes“ und nicht zuletzt auch „schlechte Verarbeitung des Kindersitzes“ genannt. Zusätzlich wurden „Platzprobleme bei mehreren Kindersitzen“ sowie eigene Unzulänglichkeiten angeführt (vgl. Tabelle 15).

\begin{tabular}{|l|c|}
\hline Schwierigkeiten beim Sichern & Häufigkeit [\%] \\
\hline Kind wehrt sich bei/gegen Sicherung & 30,2 \\
\hline Kind manipuliert unterwegs die Sicherung & 15,9 \\
\hline Gurte verdrehen sich & 14,3 \\
\hline unkomfortabel in Sitzposition & 6,3 \\
\hline Kind öffnet Sicherung während der Fahrt & 4,8 \\
\hline Fangkörper verlagert sich & 3,2 \\
\hline komplizierte Gurtführung & 1,6 \\
\hline Gurtschlossposition verlagert sich & 1,6 \\
\hline weitere Probleme & 4,8 \\
\hline
\end{tabular}

Tabelle 14: Schwierigkeiten beim Sichern $(n=54)$; (Mehrfachantworten waren möglich) 


\begin{tabular}{|l|c|}
\hline $\begin{array}{l}\text { Begründungen für } \\
\text { Sicherungsprobleme }\end{array}$ & Häufigkeit [\%] \\
\hline Widerstand des Kindes & 38,9 \\
\hline benutzerunfreundlicher Kindersitz des & 11,1 \\
\hline $\begin{array}{l}\text { schlechte Verarbeitung von } \\
\text { Kindersitzes }\end{array}$ & 9,3 \\
\hline $\begin{array}{l}\text { Platzprobleme bei mehreren Kindern im } \\
\text { Auto }\end{array}$ & 9,3 \\
\hline $\begin{array}{l}\text { zu lockere Gurte aufgrund von } \\
\text { Kleidungswechsel }\end{array}$ & 7,4 \\
\hline \begin{tabular}{l} 
unbewusste Nachlässigkeit \\
\hline Kind hat (mit-)gesichert information(en)
\end{tabular} & 3,7 \\
\hline $\begin{array}{l}\text { falsche wegen } \\
\text { Herstelleranleitung }\end{array}$ & 1,9 \\
\hline $\begin{array}{l}\text { Platzprobleme } \\
\text { Gepäck/Personen }\end{array}$ & 1,9 \\
\hline Nachlässigkeit wegen kurzer Strecke & 1,9 \\
\hline weitere Gründe & 11,1 \\
\hline
\end{tabular}

Tabelle 15: Begründungen für Sicherungsprobleme ( $n=54)$; (Mehrfachantworten waren möglich)

Wie schon beim Einbau orientierten sich die Interviewpartner beim Sichern am häufigsten an der Herstelleranleitung (in über der Hälfte der Fälle), gefolgt von der Probesicherung im Fachgeschäft (ca. ein Viertel). Etwa jeder Zehnte nutzte Tipps von Verwandten / Bekannten oder probiert einfach selbst aus.

Wissen über KSS sowie Beurteilung der Schutzwirkung von KSS.- Nahezu allen Befragten war bekannt, dass es eine gesetzliche Sicherungspflicht für Kinder in speziellen Kindersitzen gibt. Wie Verstöße gegen diese gesetzliche Sicherungspflicht geahndet werden, konnte allerdings etwa jeder Zehnte der Befragten nicht benennen. Die Frage nach den gesetzlichen Kriterien zur Sicherung der Kinder brachte deutliche Wissens- und Verständnisdefizite zu Tage: Während immerhin noch $80 \%$ die Größe des Kindes als Kriterium benennen konnten, waren es hinsichtlich Alter des Kindes lediglich 71,9\% und hinsichtlich Gewicht des Kindes nur noch $55 \%$. Das lässt sich nach wie vor als (den schon früher mehrfach geäußerten) Hinweis deuten, dass die KSS-Typologie mit ihren vielen Unterformen von den Nutzern nach wie vor nur schlecht verstanden wird. 
Als sehr positive Entwicklung sind demgegenüber die Antworten auf die weiteren gestellten Fragen zu bewerten: So kannten alle Befragten die Bedeutung von „Der Kindersitz ist rückwärts einzubauen“. Gleiches gilt für die Frage, was bei der Sicherung von Kindern auf dem Beifahrersitz zu beachten ist, wenn ein Beifahrer-Airbag vorhanden ist. Auch hinsichtlich der Bekanntheit von ISOFIX-Systemen hat sich viel getan: knapp $90 \%$ der Interviewpartner war ISOFIX bekannt. Genau umgekehrt verhält es sich allerdings hinsichtlich ECE-R 129: etwa $90 \%$ der Befragten war diese neue gesetzliche Regelung nicht bekannt.

Der Großteil (92 \%) aller Befragten war von der Schutzwirkung des KSS überzeugt, der Rest nur „zum Teil“. Wurden Zweifel an der Schutzwirkung von Kindersitzen geäußert, so begründeten dies die Befragten damit, dass insbesondere bei großen Sitzen keine richtige Sicherung möglich sei.

Insgesamt lässt sich hier festhalten: Die große Mehrheit der Befragten ist von der Schutzwirkung der Kindersitze überzeugt, weist also ein positives Sicherheitsbewusstsein auf. Trotz der z.T. guten Kenntnisse zu einigen der genannten Fragestellungen weisen die Befragten dennoch zu zentralen Fragen der korrekten Handhabung der KSS hinsichtlich Einbau und Sicherung z.T. deutliche Wissensdefizite auf.

Im Folgenden soll noch auf eine Reihe weiterer Fragen eingegangen werden, die wegen kleiner Fallzahlen nur vorsichtig interpretiert werden können. So war in der telefonischen Nachbefragung auch vorgesehen, soziodemographische Daten wie etwa Bildungsstand und Haushaltseinkommen zu erheben. Dazu war allerdings jeweils weniger als die Hälfte der Befragten bereit. So läßt sich hier lediglich als vage Tendenz formulieren: Die Misusequote scheint nicht von Bildungsstand oder Einkommen abhängig zu sein. Umgekehrt erscheint wichtiger, wie gut der Sensibilisierungsgrad und auch der Informationsstand der Nutzer hinsichtlich guter Sicherung in KSS ist - auch wenn das, wie erwähnt, nicht immer zu guter Sicherung führt.

Weitere systematische Erfahrungen (die also nicht unmittelbar abgefragt worden waren) bei den Vor-Ort-Beobachtungen ergaben sich hinsichtlich folgender Punkte:

- ISOFIX-Sitze lassen sich zwar gut einbauen und vermitteln den Nutzern ein sicheres Gefühl. Sie werden allerdings als schwer und relativ unhandlich und nicht zuletzt als teuer empfunden.

- Kam das Gespräch auf das Thema Fachberatung, so zeigt sich: Auch vermeintliche Experten (Verkäufer, Hebammen, ...) werden als falsch beratend wahrgenommen. 
- Fangkörper werden von Eltern und Kindern häufig als einengend empfunden. Das führt einerseits zum Weglassen des Fangkörpers und andererseits zur falschen Nutzung von KSS: Gruppe 1-Sitze werden somit verfüht zu Gruppe 2/3-Sitzen.

- Kinder wechseln somit zu früh in die nächste KSS-Klasse.

- Das an manchen KSS installierte akustische Feedback ist bisweilen irreführend oder sogar falsch.

- Anleitungsvideos zur KSS-Installation werden als positiv und hilfreich erwähnt. 


\section{Mögliche Maßnahmen / Verbesserungsvorschläge und Empfehlungen}

In den mehrfach erwähnten früheren Studien sind aus den jeweiligen empirischen Ergebnissen bereits systematisch Empfehlungen und Forderungen an die für die Sicherheit von Kindern in Pkw maßgeblich verantwortlichen Akteure abgeleitet worden. Eine Reihe dieser Empfehlungen ist im Laufe der Jahre umgesetzt worden; sie haben zweifellos zu der in der aktuellen Studie festgestellten verringerten Misuse-Quote beigetragen. Dennoch sind einige Empfehlungen hinsichtlich der Misuse-Problematik nicht oder noch nicht umgesetzt worden. Deshalb werden im Anschluss einige dieser früheren Empfehlungen in die aktuellen Maßnahmenvorschläge integriert und es werden weitere Empfehlungen ausgesprochen.

Die Empfehlungen richten sich an:

- KSS- und Fahrzeughersteller

- Gesetzgeber und Exekutive

- Forschung und Verbraucherschutz.

\section{Empfehlungen an KSS- und Fahrzeughersteller}

Wie die aktuelle Studie zeigt und somit die früher formulierten Empfehlungen bestätigt, reduzieren ISOFIX-Vorrichtungen drastisch das Misuse-Potential beim Einbau von KSS. Deshalb muss die Verbreitung von ISOFIX weiter gefördert werden: Kindersitze, bei denen Einbau und Sichern zwei getrennte Vorgänge darstellen, sollten standardmäßig ausschließlich mit ISOFIX hergestellt werden.

Gebrauchsanleitungen und Piktogramme werden zwar von der großen Mehrheit der KSSNutzer sowohl hinsichtlich der Verständlichkeit als auch hinsichtlich des Informationsgehalts positiv beurteilt, zugleich sind jedoch viele Nutzer nicht in der Lage, Fehlbedienungen als solche zu erkennen. Dies verweist auf eine mangelnde Handlungsrelevanz dieser Anleitungen, die daher zu erhöhen ist. Zur Optimierung von Verständlichkeit und Nachvollziehbarkeit sollten Gebrauchsanleitungen und Piktogramme so gehalten und gestaltet sein, dass mit ihrer Hilfe jeder "naive“ Nutzer den betreffenden Kindersitz korrekt bedienen kann.

Da Fehlbedienungen niemals völlig auszuschließen sind, sollte potentiellen Fehlerquellen größerer Raum in schriftlichen oder filmischen Gebrauchsanleitungen eingeräumt werden. Dabei ist nicht nur auf Misuse-Quellen (wie z.B. beim Kleidungswechsel) aufmerksam zu machen, sondern es sollten auch Strategien zur Vermeidung oder Korrektur von Misuse angesprochen werden. Dabei sind insbesondere die wichtigsten 
Fehlbedienungsmöglichkeiten $\mathrm{zu}$ thematisieren. Weiterhin sollte eindrücklich gezeigt werden, welche Folgen bereits scheinbar kleinere Fehler nach sich ziehen können.

$\mathrm{Ab}$ einem gewissen Alter setzen sich Kinder mit ihrem Kindersitz aktiv auseinander, wollen es sich bequem(er) machen oder auch einfach nur ausprobieren, wie das alles so funktioniert. Manipulationen der Kinder an der Sicherung sind daher eine bedeutsame Misuse-Quelle.

Zur Vermeidung von Manipulationen am Gurtschloss durch Kinder sollte dieses mit einer Vorrichtung ausgestattet sein, die als „Kindersicherung“ derartiges verhindert. Denkbar wäre auch die Anbringung eines Sensors, der solche Manipulationen erfasst und an den Fahrer meldet.

Probleme mit den Gurten - insbesondere Gurtlose - sind die am häufigsten auftretenden Misuse-Quellen. Deshalb sollten die Systeme zur Vermeidung von Gurtlose im Fahrzeuggurt und im KSS-Gurt verbessert und entsprechend verbreitet werden.

Die beiden nächsten Empfehlungen basieren nicht auf den empirisch erhobenen Daten der vorgestellten Feldstudie, resultieren aber aus systematischen Beobachtungen und Erfahrungen der Interviewer vor Ort. Deshalb sollen sie hier nicht unerwähnt bleiben.

Von befragten Personen war häufiger die Rückmeldung zu hören, dass sie sich, wenn vorhanden, Anleitungsvideos zum Einbau des KSS und zur Sicherung des Kindes angesehen und diese als sehr hilfreich empfunden hätten. Andere Personen haben sich ausdrücklich gewünscht, dass es solche Videos auch für ihr genutztes Kindersitzmodell gäbe. Es wird daher empfohlen, dass es für alle Kindersitzmodelle leicht verständliche und leicht zugängliche Anleitungsvideos gibt, die die Nutzung des KSS nachvollziehbar erklären.

Da Gurtlose im sitzeigenen Gurtsystem als ein dominantes Problem bei der Sicherung der Kinder erkannt wurde, muss nach Lösungen gesucht werden, die einerseits die Gurtlose verhindern (s.o.), zum anderen müssen sie sicherstellen, dass auch beim Auftreten von Gurtlose ein Mindestschutz des Kindes gewährleistet ist. Eine Möglichkeit dafür bieten sogenannte Chest Clips. Dieser Clip verbindet die beiden Schultergurte des Gurtes im Kindersitz und verhindert so, dass die Gurte über die Schultern des Kindes rutschen und dieses nicht mehr zurückgehalten werden kann. Auch wenn durch die Gurtlose eine große Vorverlagerung gegeben sein kann, wird das Kind grundsätzlich immer noch zurückgehalten. 


\section{Empfehlungen an Gesetzgeber und Exekutive}

Piktogramme an KSS sind wie erwähnt wichtige handlungsrelevante Informationsquellen für die Nutzer. Der Gesetzgeber ist aufgerufen, hier für verbindliche Anforderungen an Piktogramme auf KSS zu sorgen.

Eine wichtige Rolle bei der Informationsbeschaffung durch die KSS-Nutzer besitzen zweifellos Medien. Allerdings erweisen sich die dabei vermittelten Informationen häufig als verhältnismäßig einseitig, weil sie sich vornehmlich auf Themen wie z.B. Crashtests konzentrieren. Bei den Zulassungstests sollte also nicht nur dieser Aspekt vorgeschrieben sein, sondern es sollten weitere Anforderungen definiert werden wie z.B.: Kriterienkatalog für geeignete KSS, Katalog zentraler Fehlbedienungsmöglichkeiten, Kriterien der Gebrauchstauglichkeit von KSS. Zudem sollte versucht werden, diese einseitige Ausrichtung der Berichterstattung in den Medien durch geeignete Maßnahmen (z.B. regelmäßige Newsletter an die für diesen Bereich zuständigen Journalisten, entsprechende Workshops für diese Berufsgruppe) aufzubrechen.

\section{Empfehlungen für Forschung und Verbraucherschutz}

Fachgeschäfte und Fachberater spielen eine wichtige Rolle sowohl bei der Informationsbeschaffung vor dem Erwerb eines Kindersitzes als auch bei dessen Anschaffung selbst. Zugleich verweisen die Ergebnisse sowohl der aktuellen als auch der früheren Studien darauf, dass die Gesichtspunkte, die in fachlichen Beratungen zur Sprache kommen, sehr uneinheitlich sind und häufig nicht alle relevanten Aspekte thematisiert werden.

Es sollten in Zusammenarbeit mit den Industrie- und Handelskammern verbindliche Standards für die Durchführung von Fachberatungen im Vorfeld des Erwerbs von Kindersitzen entwickelt werden, die neben allgemeinen Kenntnissen zu Fragen der Sicherung selbstverständlich auch Wissen um systemspezifische Besonderheiten enthalten müssten. Besonderer Wert und Nachdruck sollte dabei auch auf die Misuse-Problematik gelegt werden. Dabei ist es nicht mit generellen Hinweisen getan; vielmehr sollten an den jeweiligen Kindersitzen, für die ein Kunden- bzw. Kaufinteresse besteht, die spezifischen Möglichkeiten von Fehlbedienungen und deren Vermeidung demonstriert werden. Eine solche Vorgehensweise würde zugleich einen Probeeinbau zum selbstverständlichen Bestandteil einer jeden fachlichen Beratung machen.

Ausländische Mitbürger standen zwar nicht im Fokus der aktuellen Untersuchung, wie aber systematische Beobachtungen im Rahmen der Felderhebungen zeigen, müssen ausländische Eltern (insbesondere südost- und osteuropäischer Herkunft) nach wie vor als besondere Problemgruppe in der Kindersicherung betrachtet werden. Dies betrifft nicht nur 
die Misuseproblematik, sondern auch die Nichtbeachtung der besonderen Sicherungspflicht von Kindern unter 12 Jahren. Dazu müssen bei ausländischen Nutzern besondere Aspekte berücksichtigt werden: Zum einen ihre soziokulturelle Heterogenität sowie Sprachbarrieren. Zum anderen müssen die in diesen Gemeinschaften anerkannten Autoritäten (z.B. Kulturtreffs, religiöse Gemeinschaft, muttersprachliche Medien) für die Legitimierung solcher Aktionen einbezogen und zugleich mit ihrer Hilfe geeignete Multiplikatoren für die Verbreitung der Aktionsinhalte gewonnen werden. Besonders hinzuweisen ist in diesem Zusammenhang auf die wichtige Rolle der Verwandten / Bekannten und insgesamt des familiären Umfeldes.

Sowohl die aktuelle als auch alle bisherigen Untersuchungen weisen darauf hin, dass seit Einführung der speziellen Sicherungspflicht für Kinder unter 12 Jahren zwar die Sicherungsquoten angestiegen sind und sich auf einem hohen Niveau stabilisiert haben, die fehlerhafte Handhabung der Schutzsysteme allerdings immer noch fester Bestandteil ihrer Nutzung ist. Hinzu kommt, dass gesetzliche Änderungen, neue technische Richtlinien etc., die Einfluss auf die Sicherung von Kindern in KSS haben regelmäßig den Gesamtkontext der Kindersicherung im Fahrzeug beeinflussen. Deshalb erscheint es sinnvoll, Felduntersuchungen zu Misuse weiterhin in regelmäßigen Abständen durchzuführen.

Zentrale Bedeutung bei der Vermeidung oder zumindest Reduzierung von Misuse kommt dem Nutzerverhalten zu. Obwohl es im Laufe der Jahre gelungen ist, mehr Nutzer für die KSS-Problematik zu sensibilisieren und zu informieren, liegen bei vielen Nutzern immer noch unzureichende Kenntnisse darüber vor, wie eine korrekte Sicherung vorzunehmen ist oder die Konsequenzen einer falschen Sicherung zu erkennen.

Bei der inhaltlichen Gestaltung von Informations- und Aufklärungskampagnen ist die Vermittlung von allgemeinen sowie systemspezifischen Kenntnissen zur korrekten Sicherung unerlässlich. Den gleichen Stellenwert sollten aber auch Hinweise auf die Gefahr von Fehlbedienungen und deren ausführliche Darstellung einnehmen.

Abschließend sollte die systematische Untersuchung von Fangkörpersitzen vorangetrieben werden. Auch wenn diese Sitztypen insbesondere in Verbraucherschutztests mit sehr guten Testergebnissen auffallen, ist ihre Schutzwirkung nach wie vor umstritten. Abschließende Ergebnisse liegen dazu bis heute nicht vor. In dieser Studie hat sich gezeigt, dass im Zusammenhang mit Fangkörpern ein neues Problem auftaucht. Häufig werden diese bewusst oder unbewusst weggelassen, sodass das Kind nur noch unzureichend durch den Fahrzeuggurt allein gesichert ist. Hier müssen Maßnahmen ergriffen werden, die diese Art der Fehlbenutzung verhindern. 


\section{Literatur}

[Bendjellal, 2006]: Bendjellal, F.; Bennett, M.; Carine S.: „Reducing Misuse of Child Restraint Systems - An attempt to Treat Loose Harness Problem“; $4^{\text {th }}$ International Conference Protection of Children in Cars, 7-8 December 2006, München, Germany

[Besafe, 2017]:https://www.besafe.com/wp-content/uploads/2015/08/BeSafe-iZi-ModularGB.pdf; 19.04.2017

[Brämig, 2006]: Brämig, F.-H.: "Child Seat Presence and Orientation Detection CPOD (ISOcare)", $4^{\text {th }}$ International Conference Protection of Children in Cars, 7-8 Dezember 2006, München, Germany

[Casper, 2012]: CASPER project: Child Advanced Safety Project for European Roads, D3.1.2: Report on effect of misuse and related items, 2012

[Cehic, 2012]: Cehic, Eldin: "Untersuchung der Fahrzeugschnittstelle von Antirotationseinrichtungen an ISOFIX-Sitzen", Studienarbeit Nr. 04/12 an der Technischen Universität Berlin; 18.11.2012

[Cybex, 2017]:http://cybex-

online.com/media/productdetails/sirona_easy_entry_and_exit/usp/Sirona_easy_entry.pn g; 19.04.2017

[Fastenmeier, 2006]: Fastenmeier, W.; Lehnig, U.: „Fehlerhafte Nutzung von Kinderschutzsystemen in Pkw“, Bericht der BASt, Heft M178, Bergisch Gladbach, 2006

[Hummel, 2003]: Hummel, Th.; Finkbeiner, F.; Roselt, Th.: „Kinder im Auto. Studie zur Verwendung von Kinderschutzsystemen und Verbesserungspotentiale durch ISOFIX“, Berlin: Verkehrstechnisches Institut der Deutschen Versicherer, 2003

[Hummel, 2008]: Hummel, Th.; Finkbeiner F.; Kühn, M.: „Misuse of Child Restraint Systems - A 2008 Observation Study in Germany", $6{ }^{\text {th }}$ International Conference Protection of Children in Cars, 4-5 Dezember 2008, München, Germany

[Hummel, 2010]: Hummel, Th.; Finkbeiner, F.; Kühn, M.: „Fehlerhafte Nutzung von Kinderschutzsystemen - eine Beobachtungsstudie“, Berlin: UDV, 2008

[Johannsen, 2006]: Johannsen, $\mathrm{H}$.: „Assessment of Abdominal Injury Risks by Dummy Tests“; Fortschritt-Berichte VDI; Reihe 12 Verkehrstechnik / Fahrzeugtechnik, Nr. 631; VDI Verlag GmbH, Düsseldorf 2006 
[Johannsen, 2007]: Johannsen, H.: "Concepts towards Reduced Misuse Rates"; $5^{\text {th }}$ International Conference Protection of Children in Cars, 6-7 Dezember 2007, München, Germany

[Langwieder, 1997]: Langwieder, K.; Stadler, P.; Hummel, Th.; Fastenmeier, W.; Finkbeiner, F.: „Verbesserung des Schutzes von Kindern in Pkw“, Bericht der BASt, Heft M37, Bergisch Gladbach, 1997

[Müller, 2009]: Müller, G.; Borgmann, J.-P.; Johannsen, H.: "Technical options for reduction of misuse of group 1 CRS", $7^{\text {th }}$ International Conference Protection of Children in Cars, 3-4 Dezember 2009, München, Germany

[Müller, 2013]: Müller, G.:,Fehlgebrauch von Kindersicherungssystemen im Fahrzeug“. Dissertation; TU Berlin ILS FG Kraftfahrzeuge; URN: urn:nbn:de:kobv:83-opus4-43371, 2013

[Müller, G., Johannsen, H. \& Fastenmeier, W., 2010]: Müller, G., Johannsen, H. \& Fastenmeier, W.: „Fehlgebrauch der Airbagabschaltung bei der Beförderung von Kindern in Kinderschutzsystemen“, Berichte der Bundesanstalt für Straßenwesen, F 75. Bremerhaven: Wirtschaftsverlag NW, 2010 


\title{
6 Anhang
}

\subsection{Beobachtungsbogen}

Version vom 30.06.2016

GKB UG

Gesellschaft für

Kraftfahrzeugtechnik Berlin

\section{Beobachtungsbogen}

\author{
(Teil 1)
}

\section{- Nutzung von Kindersitzen im Pkw -}

Im Auftrag des GDV

\begin{tabular}{|l|l|}
\hline Fallnummer: & Schema: \\
& Erhebungsort \\
Datum & Fortlaufende Nummerierung \\
\hline
\end{tabular}

Wetter: $\square$ warm, sonnig $\square$ warm, bedeckt $\square$ kühl, sonnig $\square$ kühl, bedeckt $\square$ Niederschlag $\square$ frostig
Befragungsort: $\square$ Einkaufszentrum

$\square$ Einkaufs-/Supermarkt

$\square$ Kindergarten

$\square$ Schule

$\square$ Freizeiteinrichtung

$\square$ BAB Raststätte

$\square$ anderer Ort und

zwar:

Bei festgestellten Misuse:

- Befragung mit Fragebogen und Bitte um Teilnahme am Tiefeninterview!

- Daten von Interessenten an Interviews auf getrennter Vorlage notieren! (Datenschutz)

- Daten für telefonische Befragung auf dafür vorgesehener Vorlage notieren! (Telefonnummer und Uhrzeit/Datum)

Telefonisch erreichbar unter: , um am: Uhr 


\section{Angaben zu Fahrzeug und Insassen}

Fahrzeughersteller: Fahrzeugtyp:

Fahrzeugart:

Baujahr:

Anzahl Türen: $\square$ 2/3-türig

$\square$ 4/5-türig

$\square$ sonstiges: (z.B. Kombi) (ggf. Foto von halber Fahrzeugrückseite, ohne Nummernschild)

Beifahrerairbag: $\square$ ja $\square$ nein Seitenairbag/Vorne: $\square$ ja $\square$ nein Hinten: $\square$ ja $\square$ nein Airbags deaktiviert? $\square$ nein $\square$ ja $\rightarrow$ von wem vorgenommen?
$\square$ Fachwerkstatt
$\square$ Abschaltung
$\square$ Transponder/Erkennung
$\square$ ausgebaut von Sitzbelegung

War früher der/die Airbag(s) deaktiviert? $\square$ ja

Insassen insgesamt:

davon Kinder unter 12 Jahre:

Fahrtdauer: ca. $\min$

Fahrtzweck: $\square$ Einkauf $\square$ Arbeitsweg

$\square$ Heimweg

DErledigungen

$\square$ Verwandte / Bekannte

$\square$ Ausflug / Urlaub

$\square$ Schule/Kita

$\square$ Sonstige:

\begin{tabular}{|c|c|c|c|c|c|}
\hline \multicolumn{6}{|c|}{ 2. Angaben zur interviewten Person } \\
\hline$\square$ Vater & $\square$ Mutter & $\square$ Großeltern & & andte/Bekannte & $\square$ andere Person \\
\hline$\square$ männlich & $\square$ weiblich & Alter: & Natio & & (falls nicht deutsch) \\
\hline Deutschken & & $\square$ gut & mäßig & $\square$ schlecht & $\square$ keine \\
\hline
\end{tabular}

Alter: (JJ/MM)

3. Angaben zu Kind(ern), KSS und misuse

Geschlecht: $(m / w)$

Größe: $(\mathrm{cm})$ (o. Konfektionsgröße)

Gewicht: (kg)

\section{Sitzposition:}

vorne rechts

$\begin{array}{ll}\text { 2. Sitzreihe } & \begin{array}{l}\text { rechts } \\ \text { Mitte } \\ \text { links }\end{array} \\ \begin{array}{ll}\text { 3. Sitzreihe } & \text { rechts } \\ & \text { Mitte } \\ \text { links }\end{array}\end{array}$

\begin{tabular}{l|l|l} 
Kind 2 & Kind 3 & Kind 4 \\
\hline
\end{tabular}

(falls keine KSS-Sicherung)

ungesichert $\rightarrow$ Befragungsende!

Kind 1
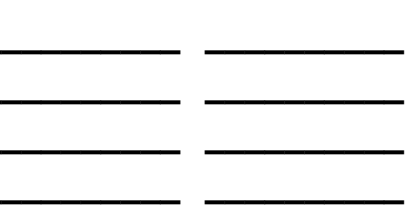

Erwachsenengurt

ebenfălls Befragungsende!

\begin{tabular}{l|l|l|l}
$\square$ & $\square$ & $\square$ & $\square$ \\
$\square$ & $\square$ & $\square$ & $\square$ \\
$\square$ & $\square$ & $\square$ & $\square$ \\
$\square$ & $\square$ & $\square$ & $\square$ \\
$\square$ & $\square$ & $\square$ & $\square$ \\
$\square$ & $\square$ & $\square$ & $\square$ \\
$\square$ & $\square$ & $\square$ & $\square$ \\
$\square$ & $\square$ & $\square$ & $\square$ \\
$\square$ & $\square$ & $\square$ & $\square$
\end{tabular}




\section{Verwendetes Schutzsystem}

ECE-Norm 44.01 / 02

ECE-Norm 44.03

ECE-Norm 44.04

ECE-Norm 129

KSS Klasse (z.B.: 0/0+/1/2/3)

KSS Firma (bitte eintragen) \&

Modellbezeichnung

\section{Einbaurichtung}

vorwärts

rückwärts

quer

Sitzreihe entgegen der Fahrtrichtung

\section{Befestigungsart des KSS}

3 PG Automatik

2 PG Automatik

2 PG-Statik

Zusatzgurt

Zusatzrückbank

integriertes KSS

ISOFIX

sonstiges und

zwar:

Bewertung des Einbaus/Sicherns

korrekt (falls für jedes Kind

zutreffend $\rightarrow$ Befragungsende!)

misuse

Fehler nach Codebook

Fehler 1

Fehler 2

Fehler 3

Fehler 4

Fehler 5

Fehler 6

Fehler 7

Fehler 8 $\square$

$\square$

$\square$

$\square$

Kind 1

$\square$

$\square$

$\square$

$\square$

Kind 3

Kind 4

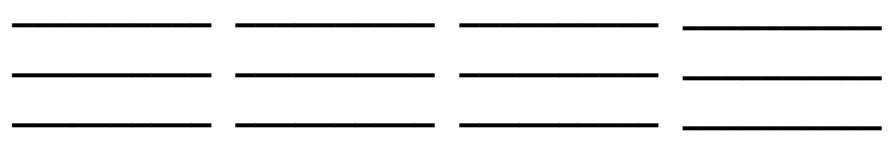

민

$\square$

$\square \quad \square$

$\square$

$\square$

$\square$

믐

$\square$

$\square$

ㅁ $\square$

ㅁ

$\square \quad \square$

ㅁ

ㅁ

$\square \quad \square$

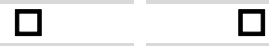

口 $\square$

문

문

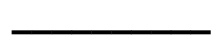

$\square$

$\square$

$\square$

$\square$

$\square$ 


\section{Fragen $z u(m)$ festgestellten misuse}

\begin{tabular}{|l|l|l|l|l|l|} 
(nur für KSS mit gesondertem Einbau) & Kind $\mathbf{1}$ & Kind 2 & Kind $\mathbf{3}$ & Kind 4 \\
\hline
\end{tabular}

Letzter Einbau von

interviewter Person

ㅁ

$\square$

$\square$

$\square \quad \square$

anderer Person und

$\square$

$\square$

zwar:

Aktuelle Sicherung von

interviewter Person

anderer Person und

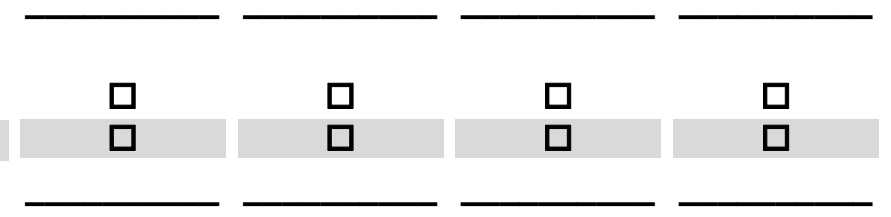

zwar:

Glauben Sie, dass alles in Ordnung/ richtig gesichert war?

ja

zum Teil

nein

weiß nicht

\begin{tabular}{l|lll}
$\square$ & $\square$ & $\square$ & $\square$ \\
$\square$ & $\square$ & $\square$ & $\square$ \\
$\square$ & $\square$ & $\square$ & $\square$ \\
$\square$ & $\square$ & $\square$ & $\square$
\end{tabular}

Wir haben festgestellt ... (interviewten

Person werden misuse gezeigt). Hat

dies Ihrer Auffassung nach negativen

Einfluss auf die KSS-Schutzwirkung?

keine Einbuße

geringe Einbuße

erhebliche Einbuße

starke Einbuße

weiß nicht

Was waren die Gründe für misuse?

unbewusste Nachlässigkeit

technisch bedingt

Komfortverbesserung für Kind

mangelnde/fehlerhafte Information

Kind sichert sich selbst

kleidungsbedingt (dick/dünn)

mehrere Kinder im Auto

kurze Strecke

Zweit-/Fremdauto, Fahrzeugwechsel

bewusste Nachlässigkeit

Widerstand des Kindes

Umständliches Einbauen/Sichern

Zeitdruck

Platzmangel wg. Gepäck/Personen

Mitfahrt bei Bekannten

Sitzwechsel unterhalb der Kinder

Unkenntnis

$\begin{array}{ll}\square & \square \\ \square & \square \\ \square & \square \\ \square & \square \\ \square & \square\end{array}$

$\begin{array}{ll}\square & \square \\ \square & \square \\ \square & \square \\ \square & \square \\ \square & \square\end{array}$

sonstige Gründe und

$\square$

$\square \quad \square$

$\square \quad \square$

$\square \quad \square$

뭄

$\square \quad \square$

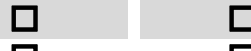

ㅁ

$\square$

$\square \quad \square$

$\square$

口

$\square$

口

ㅁ

$\square$

$\square$

zwar:

\begin{tabular}{llll}
$\square$ & $\square$ & $\square$ & $\square$ \\
$\square$ & $\square$ & $\square$ & $\square$ \\
$\square$ & $\square$ & $\square$ & $\square$ \\
$\square$ & $\square$ & $\square$ & $\square$ \\
$\square$ & $\square$ & $\square$ & $\square$ \\
$\square$ & $\square$ & $\square$ & $\square$ \\
$\square$ & $\square$ & $\square$ & $\square$ \\
$\square$ & $\square$ & $\square$ & $\square$ \\
$\square$ & $\square$ & $\square$ & $\square$ \\
$\square$ & $\square$ & $\square$ & $\square$ \\
\hline
\end{tabular}




\subsection{Codebook}

\section{CODEBOOK 2016 Fehler in KSS-Gruppe 0/0+}

\section{Allgemein}

$001 \mathrm{~S} \quad$ Kind ist zu groß für KSS

002

003

$004 \mathrm{~s} \quad$ KSS Position im Fahrzeug nicht vom Hersteller zugelassen

005 S KSS auf Beifahrersitz mit aktivem Frontairbag

$006 \mathrm{~S} \quad$ unzulässige Einbaurichtung

$007 \mathrm{~S} \quad$ KSS unbefestigt

008 LMS Sitzschiefstand

009 S KSS auf Mittelsitz positioniert (\& ungeeignet)

010 KM Geometrische Inkompatibilität mit dem Fahrzeug

011 MS Decke (o.̈̈.) unter KSS

012 KM mangelnde Seitenstabilität

013 KM kein KSS verwendet

$014 M \quad$ KSS leicht defekt/beschädigt

$015 \mathrm{~S} \quad$ KSS stark defekt/beschädigt

016 LMS keine Zulassung

017 LMS keine Zulassung für das Fahrzeug

$018 \mathrm{~L} \quad$ Vordersitz zu nah am KSS

$019 \mathrm{~S}$ eigene Konstruktion

\section{ISOFIX zur Befestigung}

020 LM Anpressdruck des KSS gering

$021 \mathrm{~S} \quad$ ISOFIX nicht eingehakt (beidseitig)

$022 \mathrm{~s}$ ISOFIX gurtschlossseitig nicht eingehakt

$023 \mathrm{~s}$ ISOFIX gurtankerseitig nicht eingehakt

$024 \mathrm{~S}$

$025 M$

$026 \mathrm{~S}$

$027 \mathrm{M}$

$028 \mathrm{~S}$

029 LMS

030 LMS

$031 \mathrm{~L}$

$032 \mathrm{~L}$

$033 \mathrm{~S}$

$034 \mathrm{KM}$
ISOFIX ohne TopTether verwendet

TopTether lose

ISOFIX ohne Stützfuß verwendet

Stützfuß nicht gespannt/lose

Stützfuß auf Staufach

\section{Fahrzeuggurt zur Befestigung}

\section{Gurtlose des Beckengurtes}

Gurtlose des Schultergurtes

Fahrzeuggurt verdreht

falsches Gurtschloss genutzt

Fahrzeuggurtschloss geöffnet

Fahrzeuggurt zu kurz
035 LS

036 LS

$037 \mathrm{~S}$

$038 \mathrm{~S}$

039 MS

040 MS

$041 \mathrm{~S}$

$042 M$

$043 M$

044 LMS

\section{Gurtsystem(KSS)}

045 LMS Gurtlose im KSS

$046 \mathrm{~S} \quad$ KSS-Gurtschloss im Sitz geöffnet

047 L KSS-Gurt im Sitz verdreht

048 L KSS-Gurtsystem nicht an Kind angepasst (Höhenverstellung zu niedrig)

049 M KSS-Gurtsystem nicht an Kind angepasst (Höhenverstellung zu hoch)

$050 \quad$ KSS Gurte vertauscht

051 KM Beinkontakt mit Rückenlehne

052 S Babyschale nicht eingerastet (Babyschale + Basis Kombination)

$053 \mathrm{M}$ Tragegriffposition nicht korrekt

054 M Abstützung am Armaturenbrett nicht ausreichend

056

057

Infobox

Fahrzeuggurt Gurtlose: Leicht - Weniger als eine flache Hand passt Mittel- zwischen KSS und Fahrzeugsitz Schwer Mehr als passt dazwischen dazwischen

KSS-Gurtlose:

Leicht - flache Hand passt gerade zwischen Gurt und Kind

Mittel - Hand passt leicht dazwischen

Schwer - Gurte können über die Schulter gezogen werden 


\section{CODEBOOK 2016 Fehler in KSS-Gruppe 1}

\begin{tabular}{|c|c|c|}
\hline & & Allgemein \\
\hline 101 & MS & 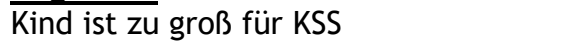 \\
\hline 102 & $\mathrm{~L}$ & Kind ist zu schwer für KSS \\
\hline 103 & LM & Kind ist zu klein für KSS \\
\hline 104 & LS & Kind ist zu leicht für KSS \\
\hline 105 & $S$ & $\begin{array}{l}\text { KSS-Position im Fahrzeug nicht vom } \\
\text { Hersteller zugelassen }\end{array}$ \\
\hline 106 & $S$ & $\begin{array}{l}\text { Rebound KSS auf Beifahrersitz mit } \\
\text { aktivem Frontairbag }\end{array}$ \\
\hline 107 & & \\
\hline 108 & $S$ & unzulässige Einbaurichtung \\
\hline 109 & $S$ & KSS unbefestigt \\
\hline 110 & LMS & Sitzschiefstand \\
\hline 111 & S & $\begin{array}{l}\text { KSS auf Mittelsitz positioniert } \\
\text { (\& ungeeignet) }\end{array}$ \\
\hline 112 & KM & $\begin{array}{l}\text { Geometrische Inkompatibilität mit dem } \\
\text { Fahrzeug }\end{array}$ \\
\hline 113 & MS & Decke (o. Ä.) unter KSS \\
\hline 114 & KM & mangelnde Seitenstabilität \\
\hline 115 & KM & kein KSS verwendet \\
\hline 116 & $M$ & KSS leicht defekt/beschädigt (Foto) \\
\hline 117 & S & KSS stark defekt/beschädigt (Foto) \\
\hline 118 & LMS & keine Zulassung \\
\hline 119 & LMS & keine Zulassung für das Fahrzeug \\
\hline 120 & $\mathrm{~S}$ & Beifahrersitz mit KSS zu weit vorn \\
\hline 121 & LMS & Beifahrersitz mit KSS hinter B-Säule \\
\hline 122 & S & Vordersitz zu nah am KSS \\
\hline 123 & $S$ & eigene Konstruktion \\
\hline & & ISOFIX zur Befestigung \\
\hline 124 & LM & Anpressdruck des KSS gering \\
\hline 125 & S & ISOFIX nicht eingehakt (beidseitig) \\
\hline 126 & S & ISOFIX gurtankerseitig nicht eingehakt \\
\hline 127 & $S$ & ISOFIX gurtschlossseitig nicht eingehakt \\
\hline 128 & $S$ & ISOFIX ohne TopTether verwendet \\
\hline 129 & M & TopTether zu lose \\
\hline 130 & $S$ & ISOFIX ohne Stützfuß verwendet \\
\hline 131 & M & Stützfuß nicht gespannt/lose \\
\hline 132 & $S$ & Stützfuß auf Staufach \\
\hline & & Fahrzeuggurt zur Befestigung \\
\hline 133 & LMS & Gurtlose des Beckengurtes \\
\hline 134 & LMS & Gurtlose des Schultergurtes \\
\hline 135 & $\mathrm{~L}$ & Fahrzeuggurt verdreht \\
\hline 136 & L & falsches Gurtschloss genutzt \\
\hline 137 & $S$ & Fahrzeuggurtschloss geöffnet \\
\hline 138 & KM & Fahrzeuggurt zu kurz \\
\hline 139 & $M$ & $\begin{array}{l}\text { Führungshilfe Beckengurt } \\
\text { gurtschlossseitig nicht eingelegt }\end{array}$ \\
\hline 140 & M & $\begin{array}{l}\text { Führungshilfe Beckengurt } \\
\text { gurtankerseitig nicht eingelegt }\end{array}$ \\
\hline
\end{tabular}

177 S Führungshilfe Schultergurt an

Gurtumlenkung nicht eingelegt

178 S Führungshilfe Schultergurt gurtschlossseitig eingelegt

179 LMS Gurtführung falsch (Foto)

180 LMS Gurtspanner nicht genutzt

$141 \quad M \quad$ Gurtklemme nicht verwendet

142 S Gurtklemme auf der falschen Seite genutzt

143 S Gurtklemme beidseitig genutzt

144 S 2-Punkt-Gurt (statisch) statt 3-Punkt-

145 S 2-Punkt-Gurt (autom.) verwendet

146 S 2-Punkt-Gurt ohne zusätzliche Abstützung verwendet

Gurtsystem (KSS

148 S Schultergurt hinter den Armen

149 S Schultergurt über den Armen

$150 \quad \mathrm{~L} \quad$ Chest-Clip geöffnet

151 S KSS-Gurtschloss im Sitz geöffnet

$152 \quad \mathrm{KM}$ KSS-Gurtschloss zu hoch positioniert

$153 \quad M \quad$ KSS-Gurt im Sitz verdreht

$154 \quad$ KSS Gurte vertauscht

155 M Gurtsystem nicht an Kind angepasst

156 LM KSS ist nicht auf Kindesgröße eingestellt

\section{Rückwärtsgerichtete KSS}

157 KM Beinkontakt mit Rückenlehne

158 S fehlende Verankerung

(Zusatzsicherungsgurte am KSS)

159 MS mangelhafte Verankerung

(Zusatzsicherungsgurte am KSS)

$\begin{array}{lll} & & \text { Misuse mit Fangkörper } \\ 160 & \mathrm{~S} & \text { Fangkörper nicht verwendet } \\ 161 & \mathrm{~S} & \text { Fangkörper verdreht } \\ 162 & \mathrm{~S} & \text { Fangkörper nicht gesichert (ISOFIX) } \\ 163 & \mathrm{~S} & \text { Fangkörper zu hoch positioniert } \\ 164 & \mathrm{~S} & \text { nur Fangkörper verwendet } \\ 165 & \mathrm{~S} & \text { falsche Gurtführung mit Fangkörper } \\ 166 & \mathrm{~S} & \begin{array}{l}\text { Beckengurt nicht in Führung des } \\ \text { Fangkörpers }\end{array} \\ 167 & \mathrm{~S} & \begin{array}{l}\text { Schultergurt nicht in Führung des } \\ \text { Fangkörper }\end{array}\end{array}$


CODEBOOK 2016 Fehler in KSS-Gruppe 2/3

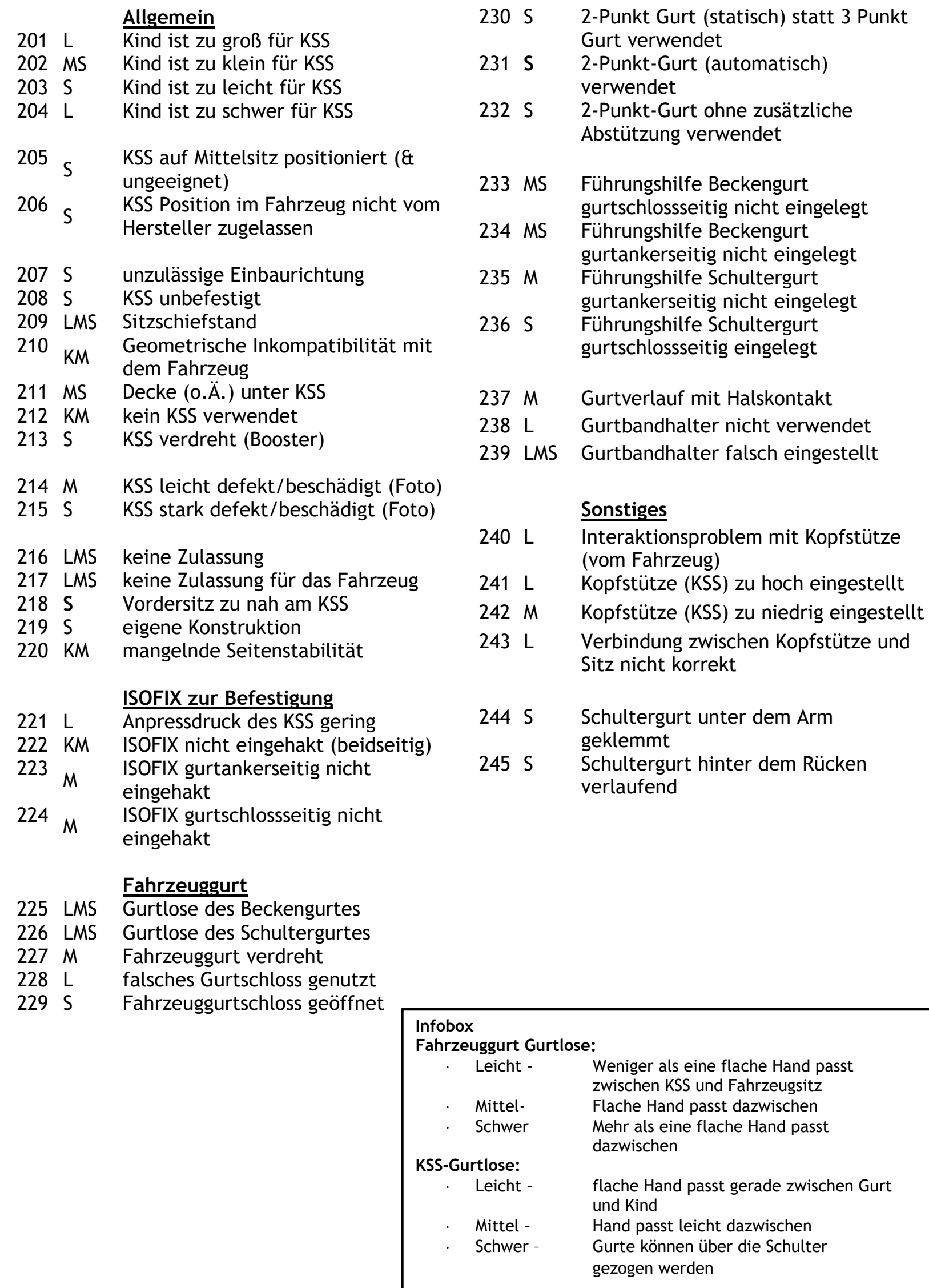




\subsection{Befragungsbogen}

GKB UG

Gesellschaft für

Kraftfahrzeugtechnik Berlin

\section{Fragebogen}

(Teil 2)

(für schriftliche oder telefonische Beantwortung)

- Nutzung von Kindersitzen im Pkw -

Im Auftrag des GDV

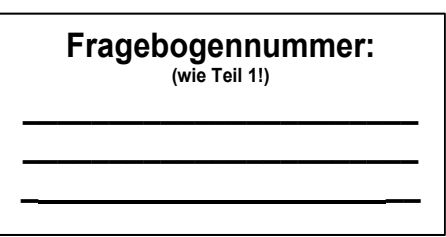

\section{INFORMATIONEN ZUM DATENSCHUTZ}

- Für die Bearbeitung von personenbezogenen Informationen gibt es das Bundesdatenschutzgesetz. Seine Vorschriften sind Grundlage der Befragung, an der Sie teilnehmen, und werden entsprechend eingehalten. Ihre Teilnahme an der Befragung ist freiwillig.

- Auf dem beiliegenden Fragebogen steht weder Ihr Name noch Ihre Adresse. Zur Erfassung der Fragebögen dient die auf dem Bogen aufgetragene Nummer.

- Die Fragebögen werden an unsere Erfassungsstelle weitergeleitet und dort mit einem Computer erfasst. Anschließend werden die Daten von uns ausgewertet. Bei der Auswertung werden Ihre Antworten ausschließlich in anonymisierter Form (d.h. ohne Name und Adresse) bearbeitet. Die Ergebnisse werden nur für Gruppen (z. B. Altersgruppen) und nicht für Einzelpersonen dargestellt. Es kann an Hand der Ergebnisse also niemand erkennen, von welchen Personen die Angaben gemacht wurden. 


\section{Fragen zum Erwerb des Kindersitzes}

\section{Position des Kindes bei der Erstbefragung}

Kind $1 \quad$ Kind $2 \quad$ Kind 3

Kind 4

1. Wann wurde der Kindersitz erworben?

(Falls nicht mehr bekannt, bitte hier ankreuzen)

2. Ist der Kindersitz ... ?

neu gebraucht gekauft ein Geschenk geliehen $\overline{(\text { Monat/Jahr) }}$ $\overline{(\text { Monat/Jahr) }}$ $\overline{(\text { Monat/Jahr) }}$

$\overline{(\text { Monat/Jahr) }}$<smiles>[LiH]</smiles>

$\square$

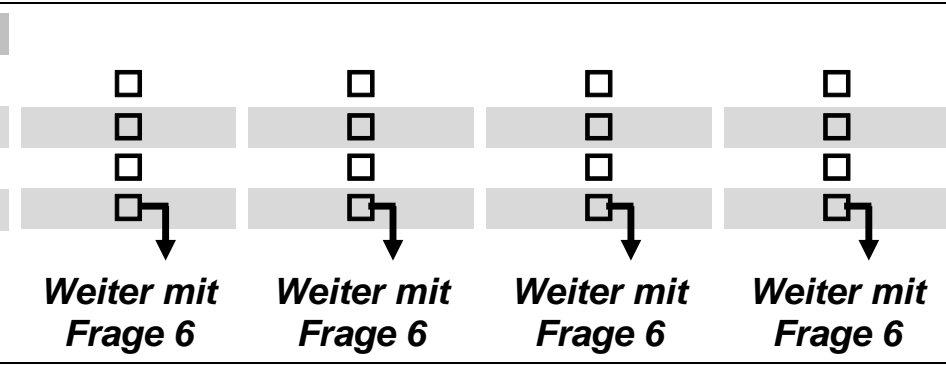

\section{Wo oder von wem wurde der Kindersitz erworben?}

Fachgeschäft

Super-/Baumarkt

Kindersitzbörse

privat (Verwandte, Bekannte)

Internet

andere Herkunft und

zwar:

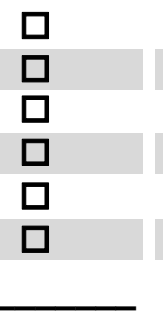

$\square$
$\square$
$\square$
$\square$
$\square$
$\square$

$\square$
$\square$
$\square$
$\square$
$\square$
$\square$

$\square$

$\square$

$\square$

$\square$

$\square$

\section{$\square$}

$\square$

$\square$

$\square$

$\square$

$\square$

4. Welcher Informationsquellen haben Sie sich vor dem Erwerb eines Kindersitzes bedient? (Mehrfachantworten sind möglich)

$\square$ Testberichte (z.B. in Zeitschriften)

$\square$ Beratung im Fachgeschäft

$\square$ Beratung durch ADAC o.ä.

$\square$ Verwandte/Bekannte

$\square$ Internet

$\square$ sonstiges und zwar:

\section{Welche Gesichtspunkte standen für Sie beim Erwerb eines Kindersitzes im} Vordergrund? (Mehrfachantworten sind möglich)
$\square$ Testergebnisse
$\square$ Tipps von Verwandten/Bekannten $\square$ Beratung im Fachgeschäft
$\square$ Hersteller/Marke
$\square$ Komfort für Kind
$\square$ ECE-Norm
$\square$ einfache Handhabung
$\square$ Eignung für Kind (Alter, Gewicht)
$\square$ Kosten / Preis
$\square$ sonstige Gesichtspunkte und zwar: 


\section{Fragen zum Einbau des Kindersitzes}

6. Worauf ist Ihrer Auffassung nach beim Einbau eines Kindersitzes zu achten? (Bitte kurz in Stichworten beschreiben)

Nachfolgende Fragen 7 bis 12 bitte nur für Kindersitze beantworten, bei denen Einbau und Sicherung zwei getrennte Vorgänge sind (Klasse 0/ 0+/1)

7. Haben Sie vor Beginn der Fahrt kontrolliert, ob der Kindersitz korrekt eingebaut war?

ja

nein

Kind 1

Kind 2

Kind 3

Kind 4

Schwierigkeiten beim Einbau

dieses Kindersitzes?

ja, manchmal

ja, öfter

nein

nehme den Einbau nicht vor

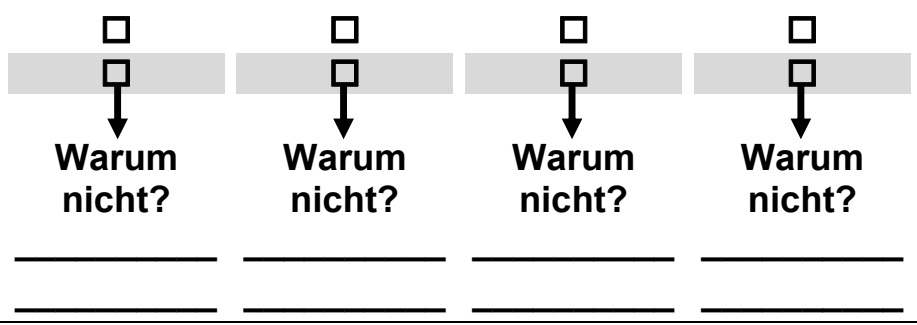

9. Welche Schwierigkeiten sind beim Einbau aufgetreten?

(Mehrfachantworten sind möglich)

komplizierte Gurtführung

Fahrzeuggurte zu kurz

aufwendiger Aus-/Einbau bei

Fahrzeugwechsel

schwer verständliche Anleitung

schlechte Passung zwischen

Kindersitz und Fahrzeug

ständige Nachprüfung der Gurte

erforderlich

fester Einbau erfordert Zusatz-

befestigung

schwere Zugänglichkeit bei

2-türigem Fahrzeug

weitere Probleme und zwar:

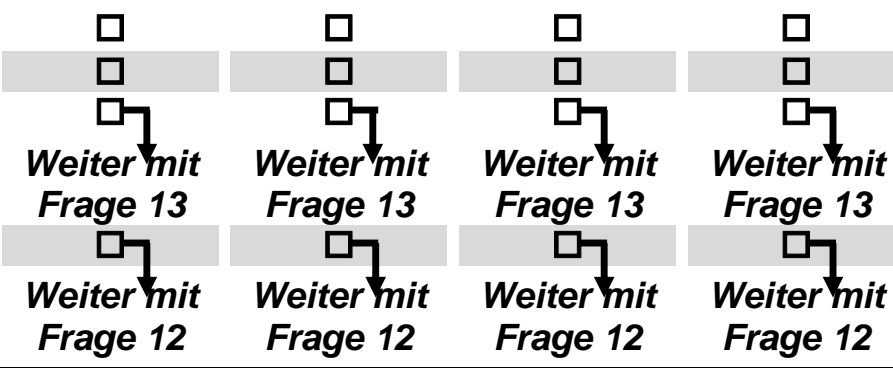

Frage 12 Frage 12 Frage $12 \quad$ Frage 12
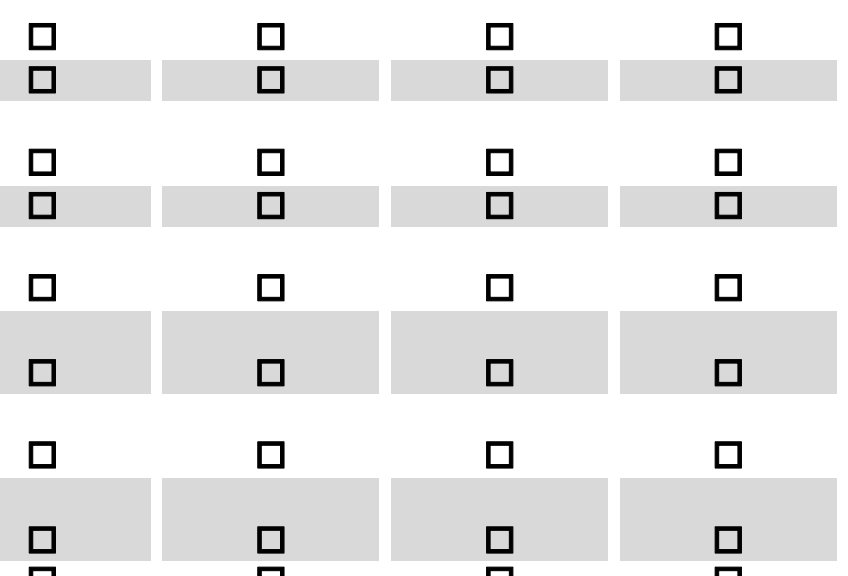

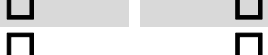

$\square$

문 $\square$

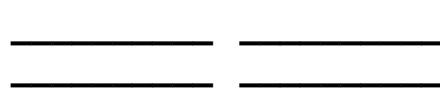


10. Worauf sind Ihrer Auffassung nach diese Probleme beim Einbau des betreffenden Kindersitzes zurückzuführen?

(Mehrfachantworten sind möglich)

Sitz nicht für Fahrzeug geeignet Fahrzeug nicht für Sitz geeignet Sitz benutzerunfreundlich falsche Information in Herstelleranleitung

fehlende Information in Herstelleranleitung eigene Ungeschicklichkeit Platzprobleme bei mehreren Sitzen Einbauprobleme wegen Zweit-I Fremdwagen weitere Gründe und zwar:

11. An welchen Informationen bzw. Hinweisen haben Sie sich beim Einbau dieses Kindersitzes hauptsächlich orientiert? (Bitte ordnen Sie bei Mehrfachantworten diese nach ihrer Wichtigkeit mit den Rangziffern 1, 2, 3 etc.)

Herstelleranleitung

Probeeinbau vor Fachgeschäft

einfach ausprobiert

Unterstützung durch Verwandte/

Bekannte

Probeeinbau vor Super-/Baumarkt

Einbau auf Basis eigener Erfahrungen

nicht mehr bekannt andere Hilfestellung und zwar:

Bitte weiter mit Frage 13!

12. Hat die Person, die den Einbau vorgenommen hat, über Schwierigkeiten berichtet?

nein

ja, und zwar,

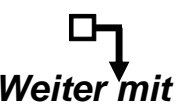

Weiter'mit

Weiter mit

$\square$

$\square$

$\square$

$\square$

$\square$

$\square$

$\square$

$\square$

$\square$

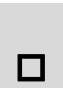

$\square$

$\square$

$\square$

$\square$

$\square$

$\square$

$\square$

$\square$

口

ㅁ

ㅁ

ㅁ

$\square$

$\square$

口

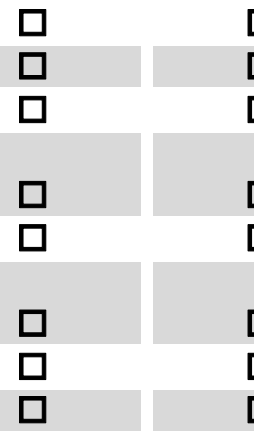

$\square$

$\square$

$\square$

$\square$

$\square$

$\square$

$\square$<smiles></smiles>

$\square$

$\square$

$\square$

$\square$

$\square$

ㅁ

$\square$

口

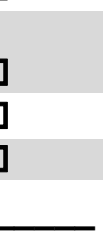

$\square$

$\square$

$\square$

$\square$

$\square$

$\square$

Frage 13

Frage 13

Frage 13

口

口

口

Weiter'mit

Frage 13

\section{dass}




\section{Fragen zum Sichern im Kindersitz}

13. Worauf ist Ihrer Auffassung nach beim Sichern eines Kindes im Kindersitz zu achten? (Bitte kurz in Stichworten beschreiben)

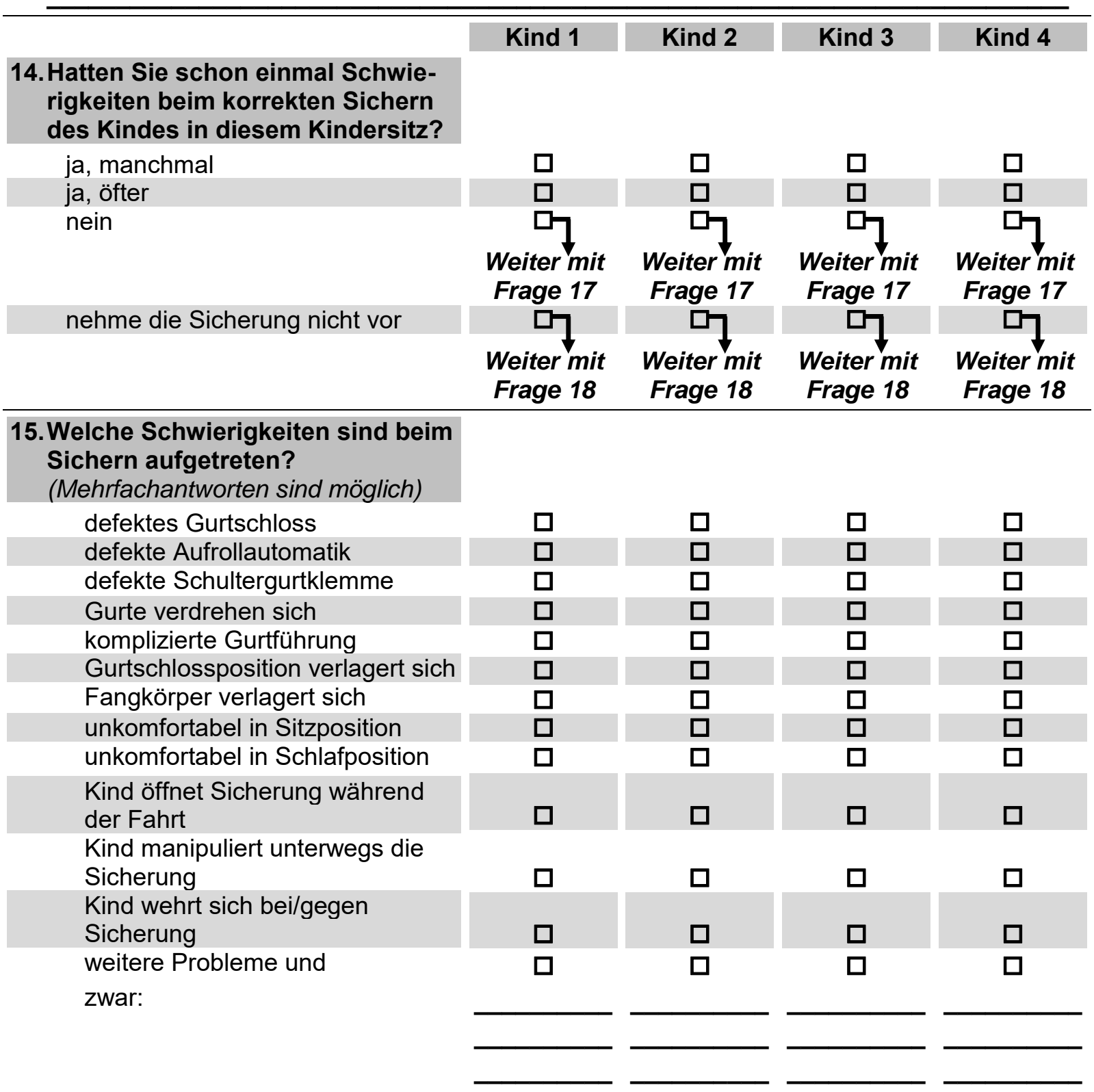


16. Worauf sind Ihrer Auffassung nach die Probleme beim Sichern zurückzuführen?

(Mehrfachantworten sind möglich)

schlechte Verarbeitung des

Kindersitzes

benutzerunfreundlicher Kindersitz

falsche Information(en) in

Herstelleranleitung

eigene Ungeschicklichkeit

fehlende Information(en) in

Herstelleranleitung

Platzprobleme wegen Gepäck/

Personen

Platzprobleme bei mehreren

Kindern im Auto

unbewusste Nachlässigkeit

zu lockere Gurte aufgrund von

Kleidungswechsel

Nachlässigkeit wegen Zeitdruck

Nachlässigkeit wegen kurzer

Strecke

vergessen, Sicherung des Kindes

zu kontrollieren

Widerstand des Kindes

Kind hat (mit-)gesichert

weitere Gründe und

zwar:

\section{Kind 1}

Kind 3

Kind 4

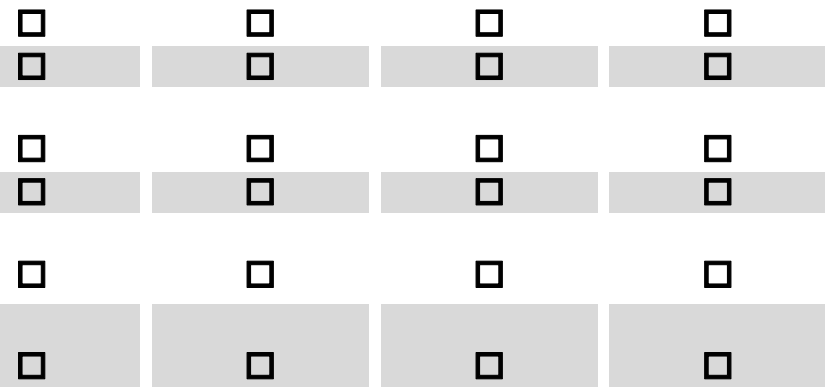

$\begin{array}{llll}\square & \square & \square & \square\end{array}$

$\begin{array}{llll}\square & \square & \square & \square\end{array}$<smiles>[B]C1[CH]C1[18O]</smiles>

$\begin{array}{llll}\square & \square & \square & \square\end{array}$

$\square$

$\square$

$\square \quad \square$

$\square \quad \square$

$\square$

$\square$

ㅁ

$\square$

$\square$

$\square$

$\square$

뭄

\section{Welche Informationen bzw. Tipps \\ haben Sie beim Sichern \\ des Kindes in diesem Kindersitz \\ hauptsächlich berücksichtigt? \\ (Bitte ordnen Sie bei Mehrfachant- \\ worten diese nach ihrer Wichtigkeit \\ mit den Rangziffern 1, 2, 3 etc.)}

Herstelleranleitung

Probesicherung vor Fachgeschäft

einfach ausprobiert
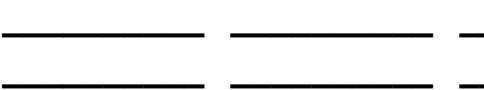

Unterstützung durch Verwandte/

Bekannte

Probesicherung vor Super-

/Baumarkt

Sicherung auf Basis eigener

Erfahrungen

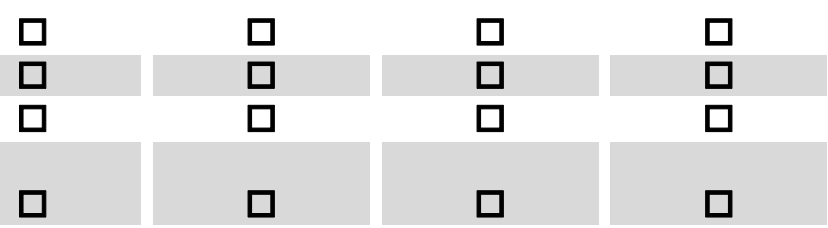

nicht mehr bekannt

andere Hilfestellung und

$\square$

$\square$

$\square$

$\square$

zwar:

Bitte weiter mit Frage 20!

$\square$

$\square$

$\square$

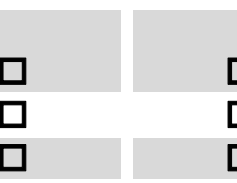

두

$\square$

$\square$

$\square$

$\square$

ㅁ

ㅁ

ㅁ 
18. Haben Sie vor Beginn der Fahrt trotzdem kontrolliert, ob das Kind korrekt gesichert war?

ja

nein \begin{tabular}{|l|l|l|l|}
\hline Kind 1 & Kind 2 & Kind 3 & Kind 4 \\
\hline
\end{tabular}

19. Hat die Person, welche die Sicherung vorgenommen hat, über

Schwierigkeiten berichtet?

nein

ja, und zwar,

dass

20.Welches der Kinder im Fahrzeug sicherte sich selbst?

$\begin{array}{lllll}\text { ja } & \square & \square & \square & \square \\ \text { nein } & \square & \square & \square & \square \\ \text { weiß nicht } & \square & \square & \square & \square\end{array}$

21. Ist eines der Kinder im Fahrzeug in der Lage, das Gurtschloss selbständig zu öffnen?

nicht bekannt

nein
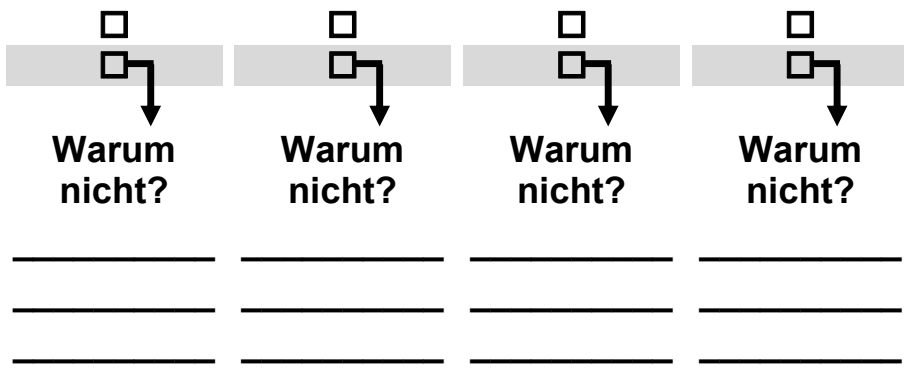


\section{IV.Allgemeine Fragen zum Sichern von Kindern im Kindersitz}

23. Ist Ihnen bekannt, dass seit einigen Jahren die Sicherung von Kindern in speziellen Kindersitzen gesetzlich vorgeschrieben ist?

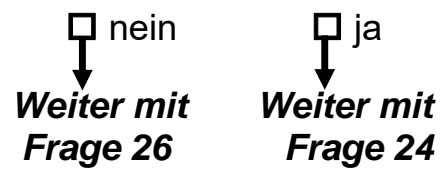

24. Was sind die gesetzlichen Kriterien, die festlegen, welche Kinder in speziellen Kindersitzen gesichert werden müssen? (Mehrfachantworten sind möglich)
$\square$ Alter
$\square$ Gewicht
$\square$ Größe

25.Werden Verstöße gegen diese gesetzliche Sicherungspflicht geahndet?
$\square$ nein
$\square$ ja

26. Für die Sicherung von Kindern gibt es verschiedene Kindersitz-Systeme (von Gruppe 0/0+ bis Gruppe III). Nach welchen Kriterien ist Ihrer Auffassung nach die Auswahl bzw. Nutzung unterschiedlicher Kindersitz-Systeme vorzunehmen? (Mehrfachantworten sind möglich)

Beachten nach welcher Norm der Sitz zugelassen ist (R44 o. R129)
$\square$ Alter
$\square$ Gewicht
$\square$ Größe
$\square$ benutztes Fahrzeug

27.Was bedeutet „Der Kindersitz ist rückwärts einzubauen“? (bitte kurz beschreiben)

28. Was ist bei der Sicherung von Kindern im Kindersitz auf dem Beifahrersitz zu beachten, wenn ein Beifahrer-Airbag vorhanden ist? (Mehrfachantworten sind möglich)

29. Wenn kein ISOFIX-Sitz in Verwendung: Kennen Sie das ISOFIX-System? (ja, nein)

$\square$ ja

$\square$ nein

Wenn ja: Warum haben Sie sich keinen ISOFIX-Sitz gekauft?

30.Wenn ISOFIX-Sitz verwendet: Warum haben Sie sich für einen ISOFIX-Sitz entschieden?

31. Wissen Sie, dass es eine neue gesetzliche Regelung zur Zulassung von Kindersitzen gibt (ECE-R 129)? (ja, nein)

$\square \mathrm{ja}$

$\square$ nein 
32. Falls 129 bekannt: Können in ihrem Auto Sitze, die nach 129 zugelassen sind, installiert werden (iSize ready)?

$\square$ ja $\square$ nein

$\square$ nicht bekannt

33. Falls 129 bekannt: Spielte die Zulassungsnorm für Ihre Kaufentscheidung eine Rolle?

$\square$ ja $\square$ nein

34.Was ist der höchste Bildungsabschluss Ihres Haushalts?

\section{Vielen Dank für Ihre Mitarbeit!}




\subsection{Struktur des Testprotokolls von EuroNCAP zur Kindersicherheit}

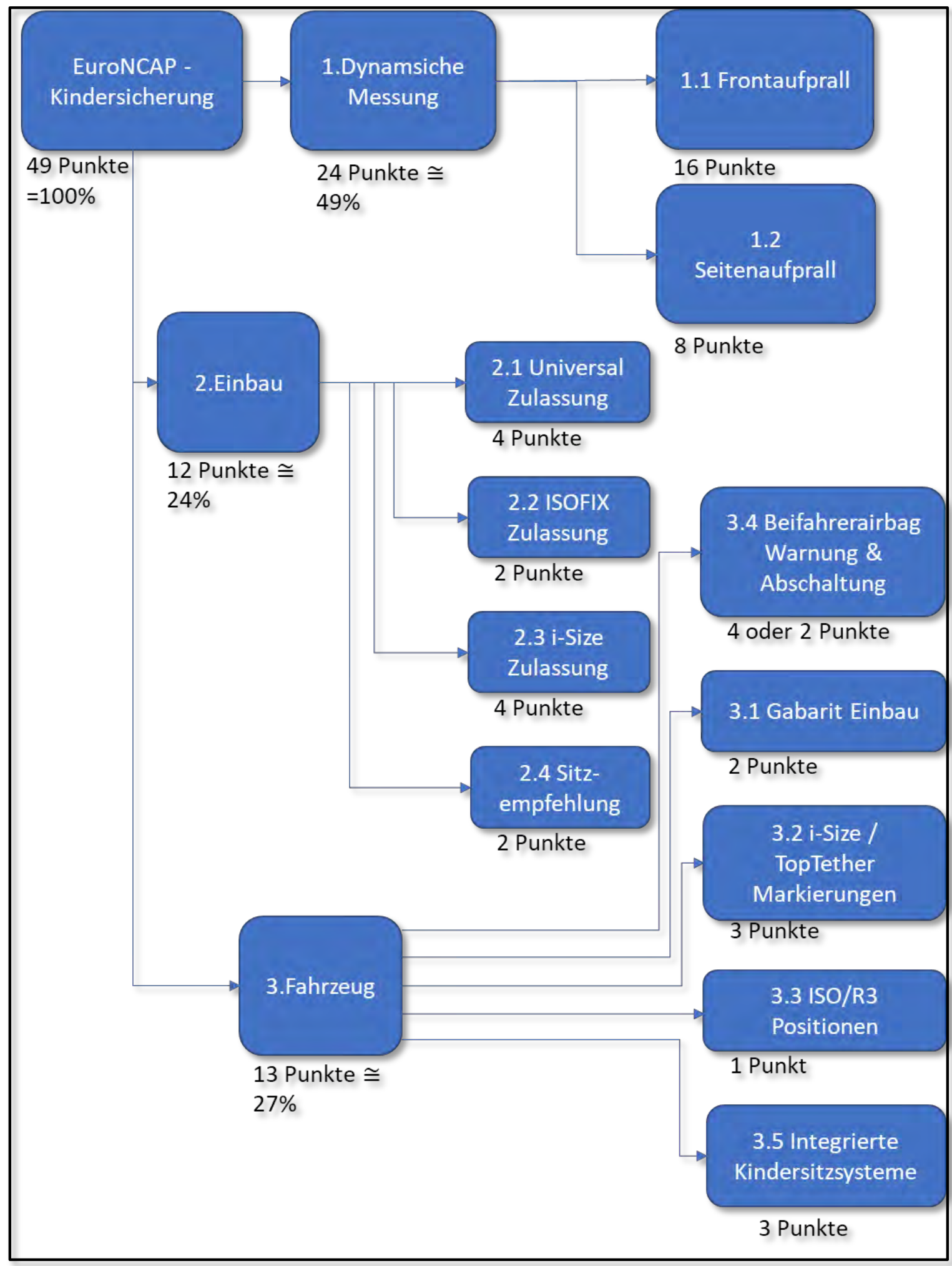


Abbildung 44: Gesamtübersicht des Testverfahrens für Kindersicherheit nach EuroNCAP 


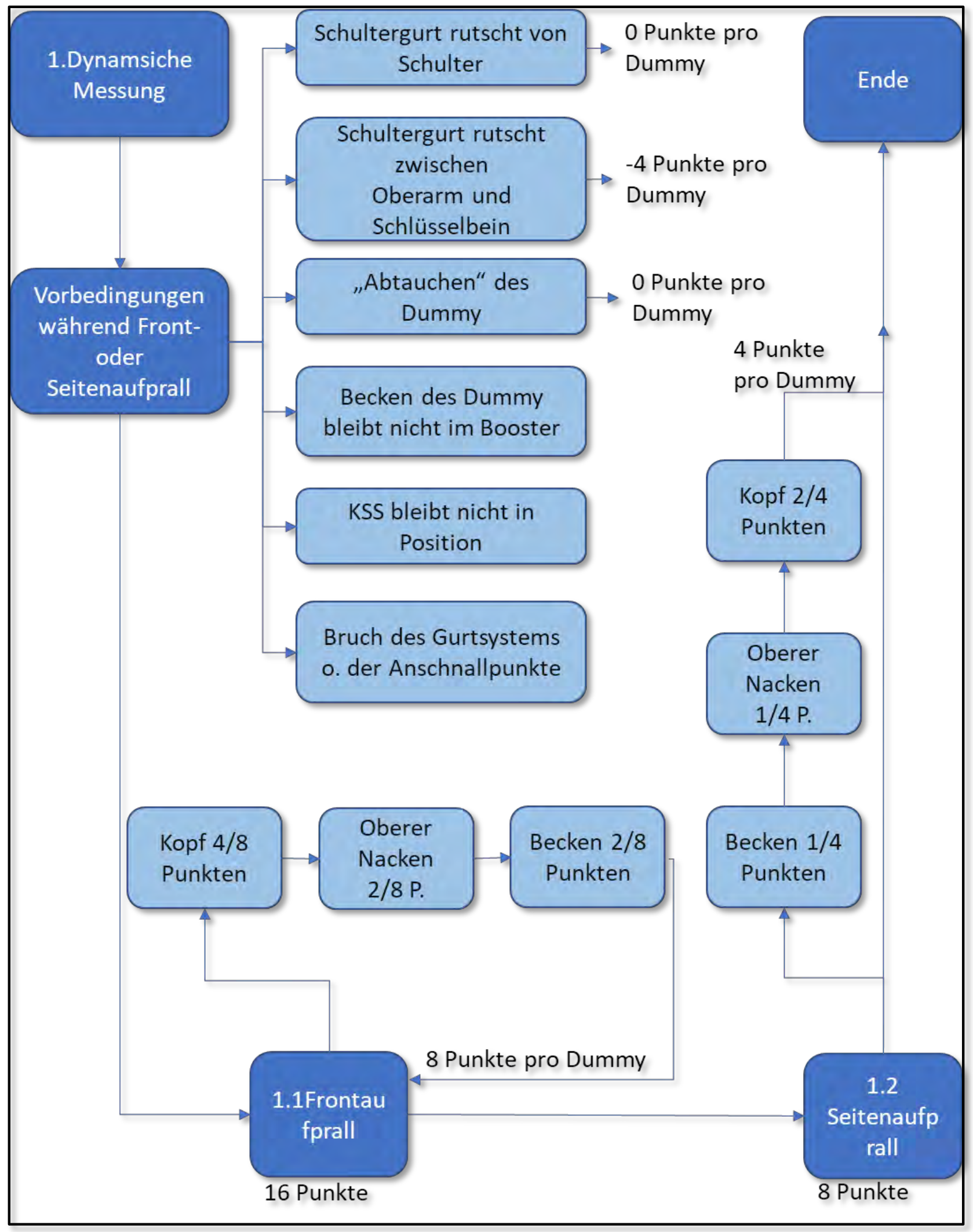

Abbildung 45: Dynamische Messungen im Bereich Kindersicherheit nach EuroNCAP 


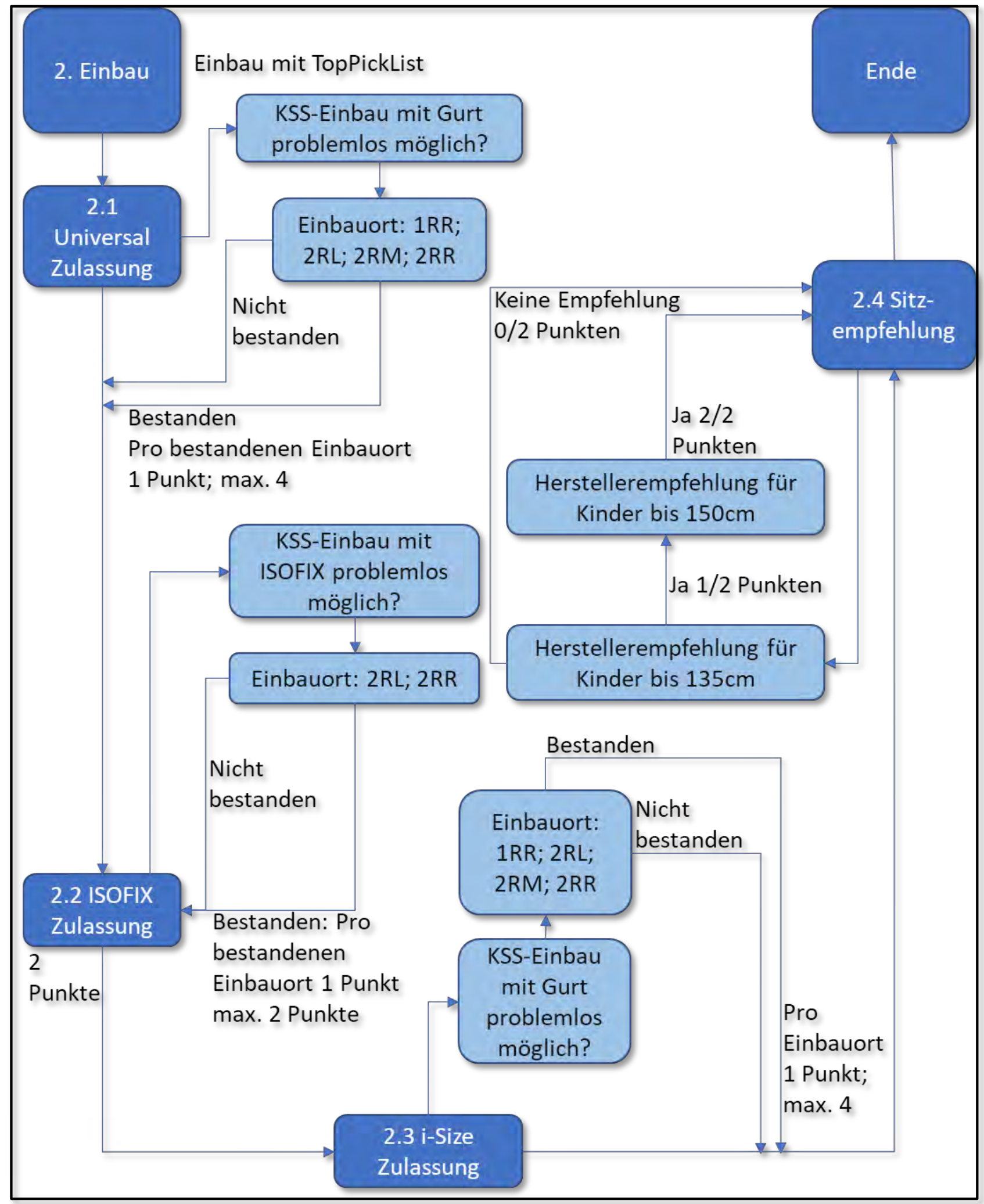

Abbildung 46: Einbauüberprüfung für Kindersitze nach EuroNCAP 


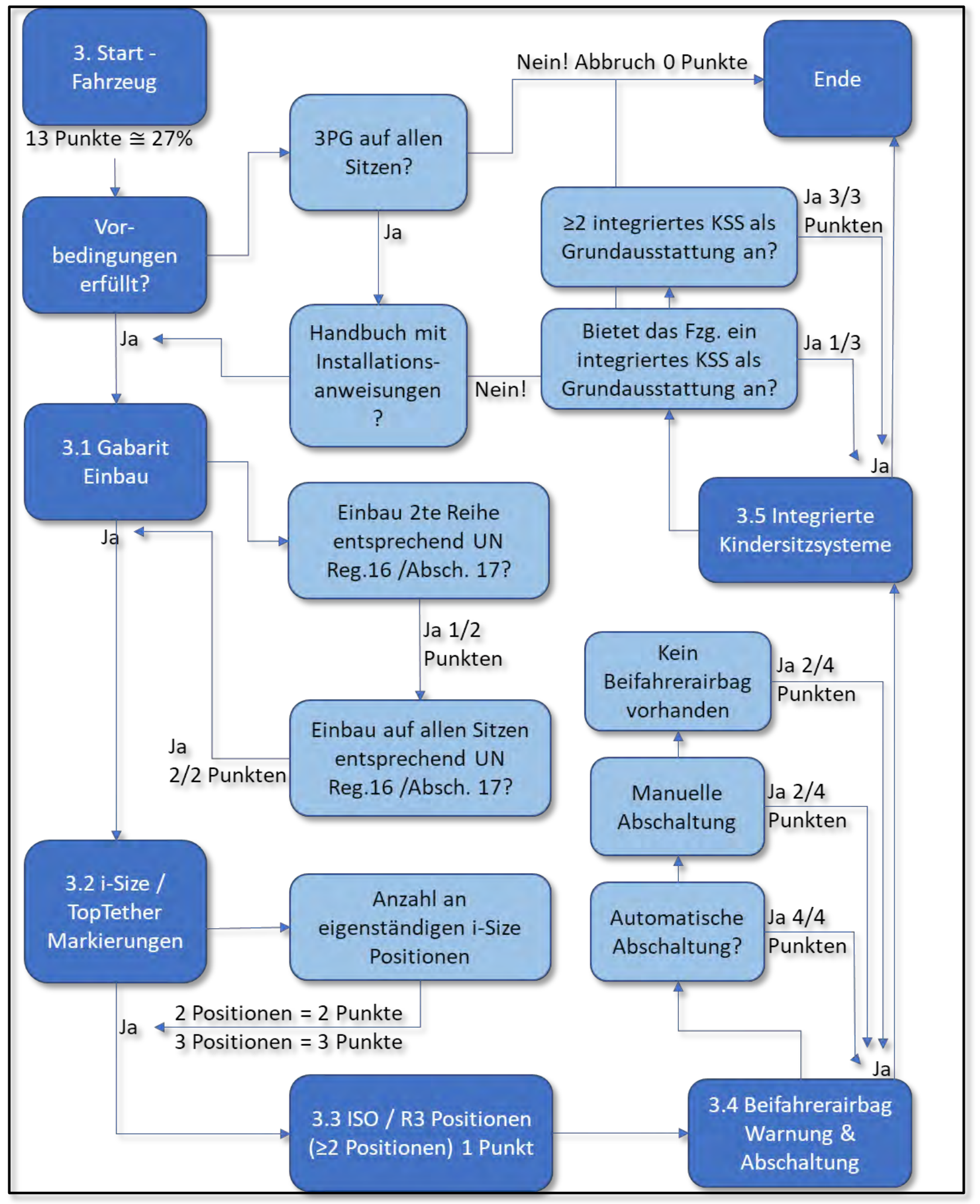

Abbildung 47: Fahrzeugbewertung im Bereich Kindersicherheit nach EuroNCAP 


\subsection{Weitere Auswertung der Feldstudie}

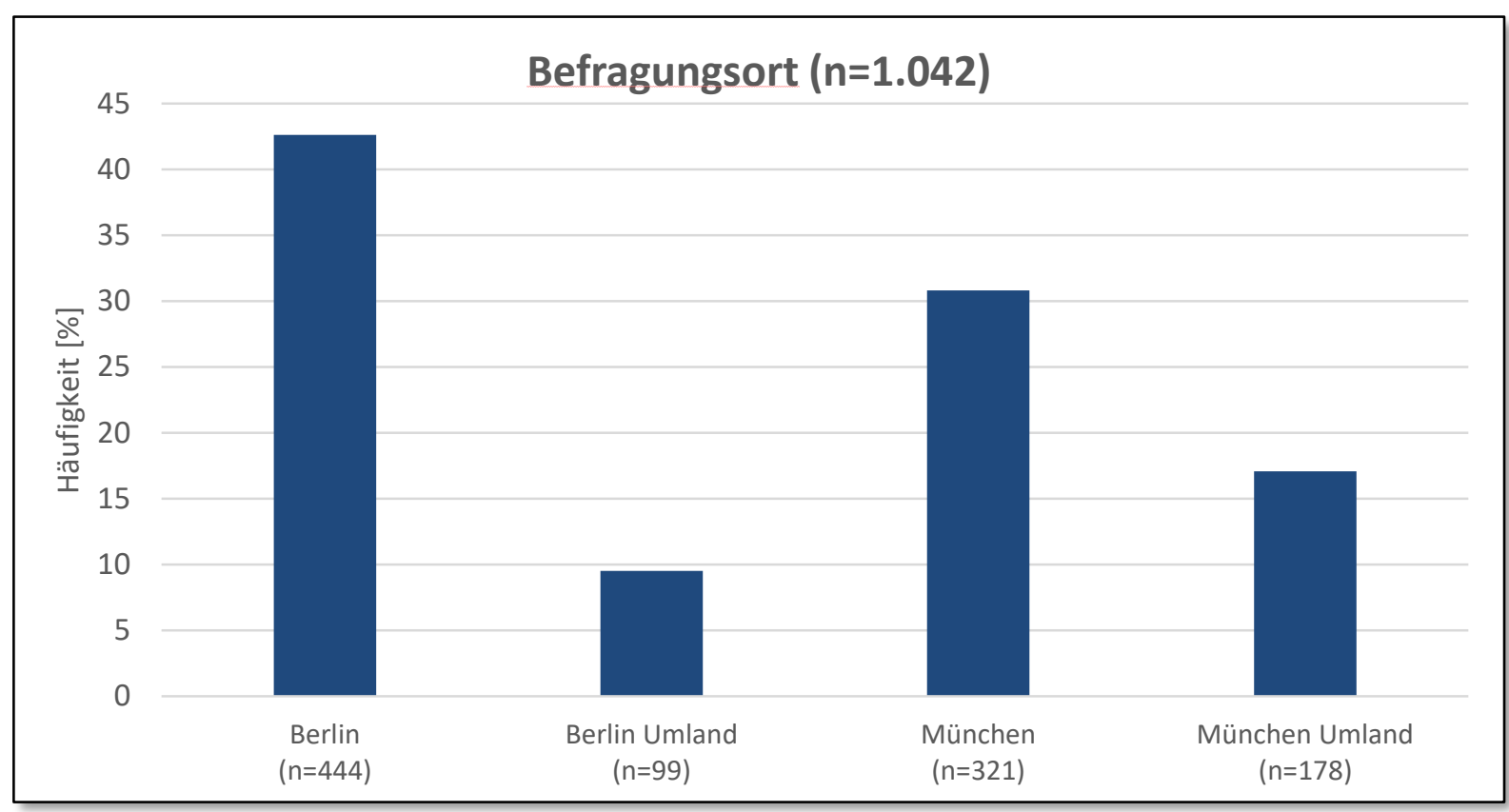

Abbildung 48: Befragungsort

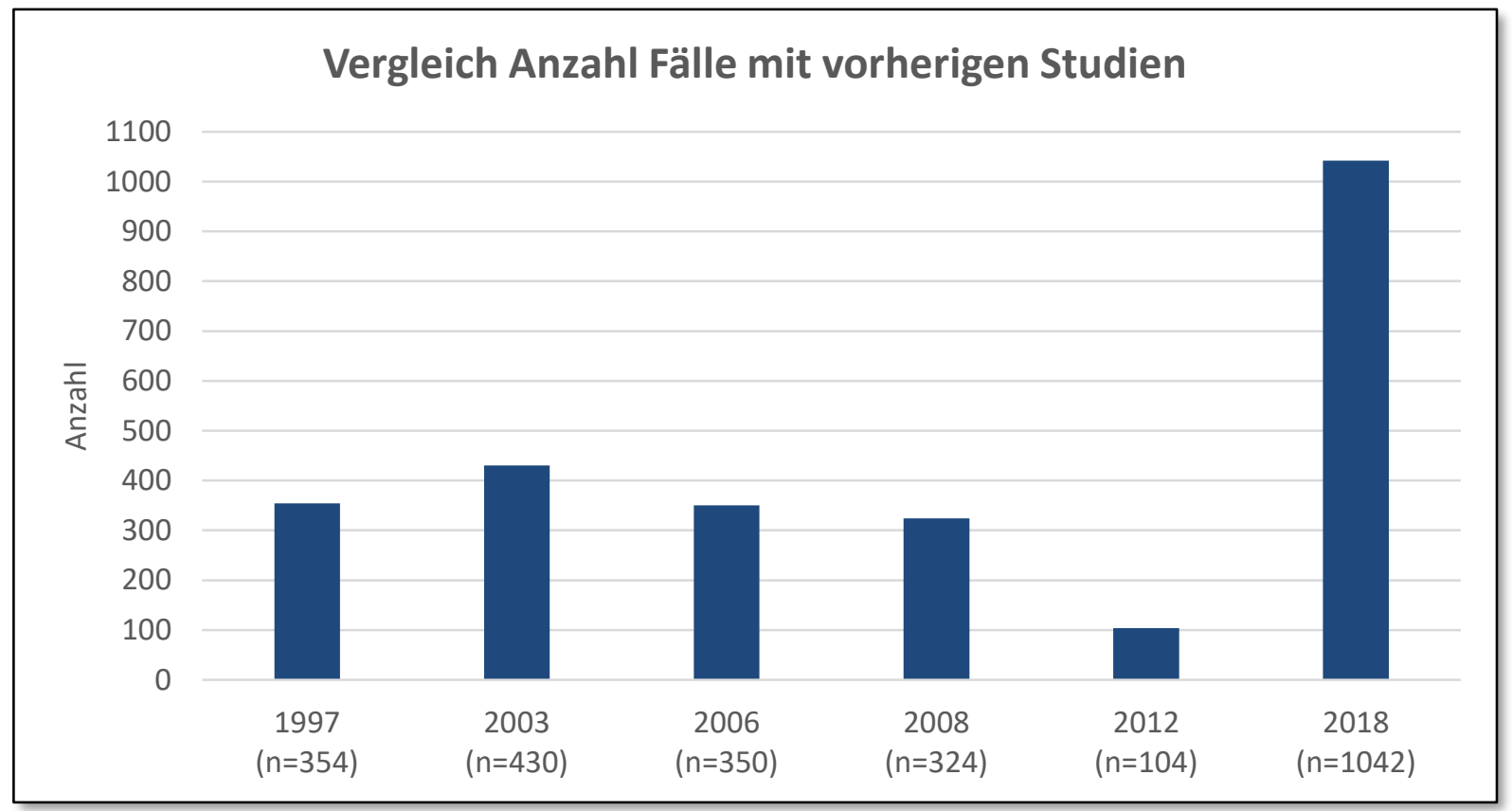

Abbildung 49: Vergleich Anzahl mit vorherigen Studien 


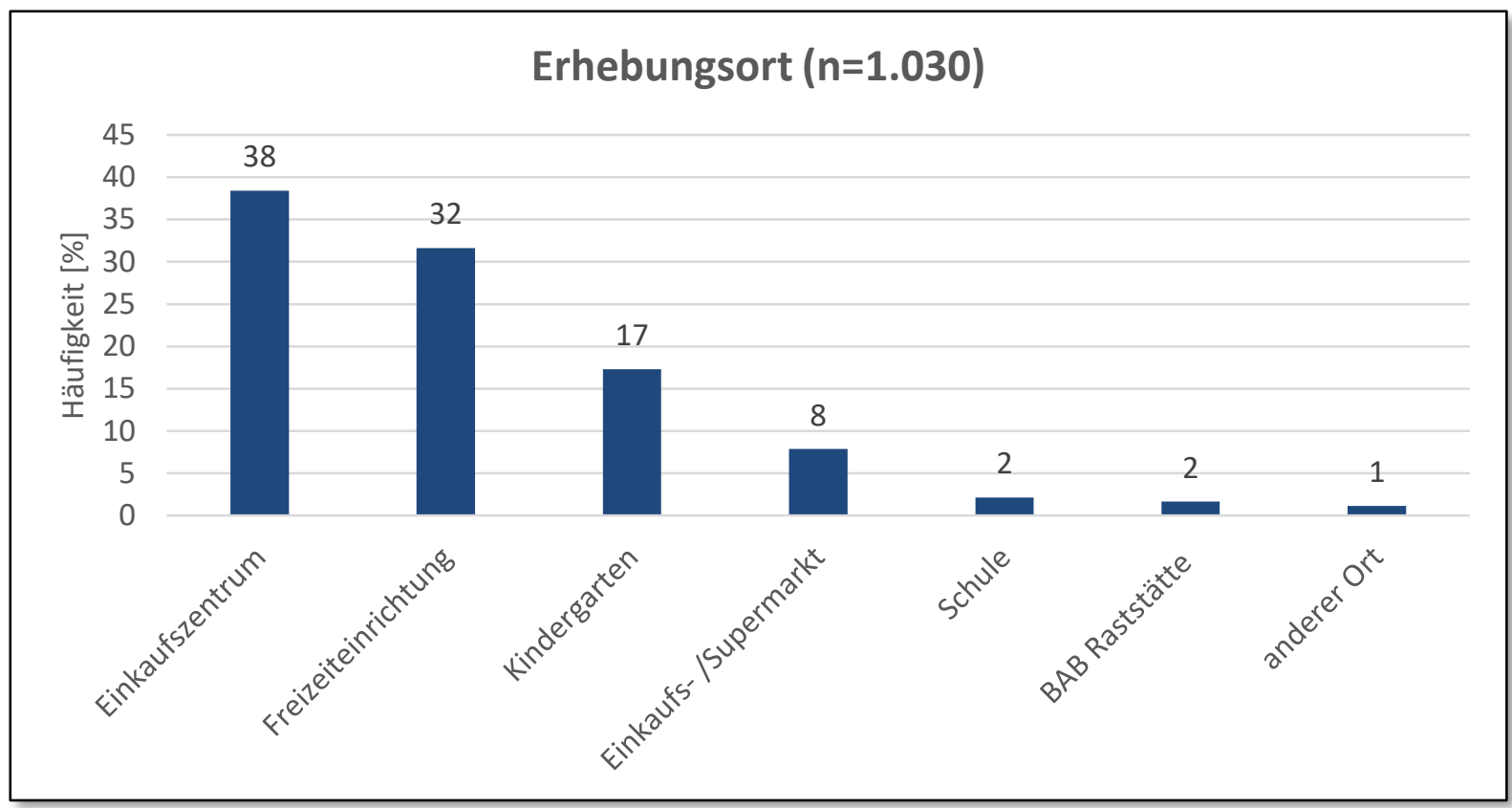

Abbildung 50: Erhebungsort

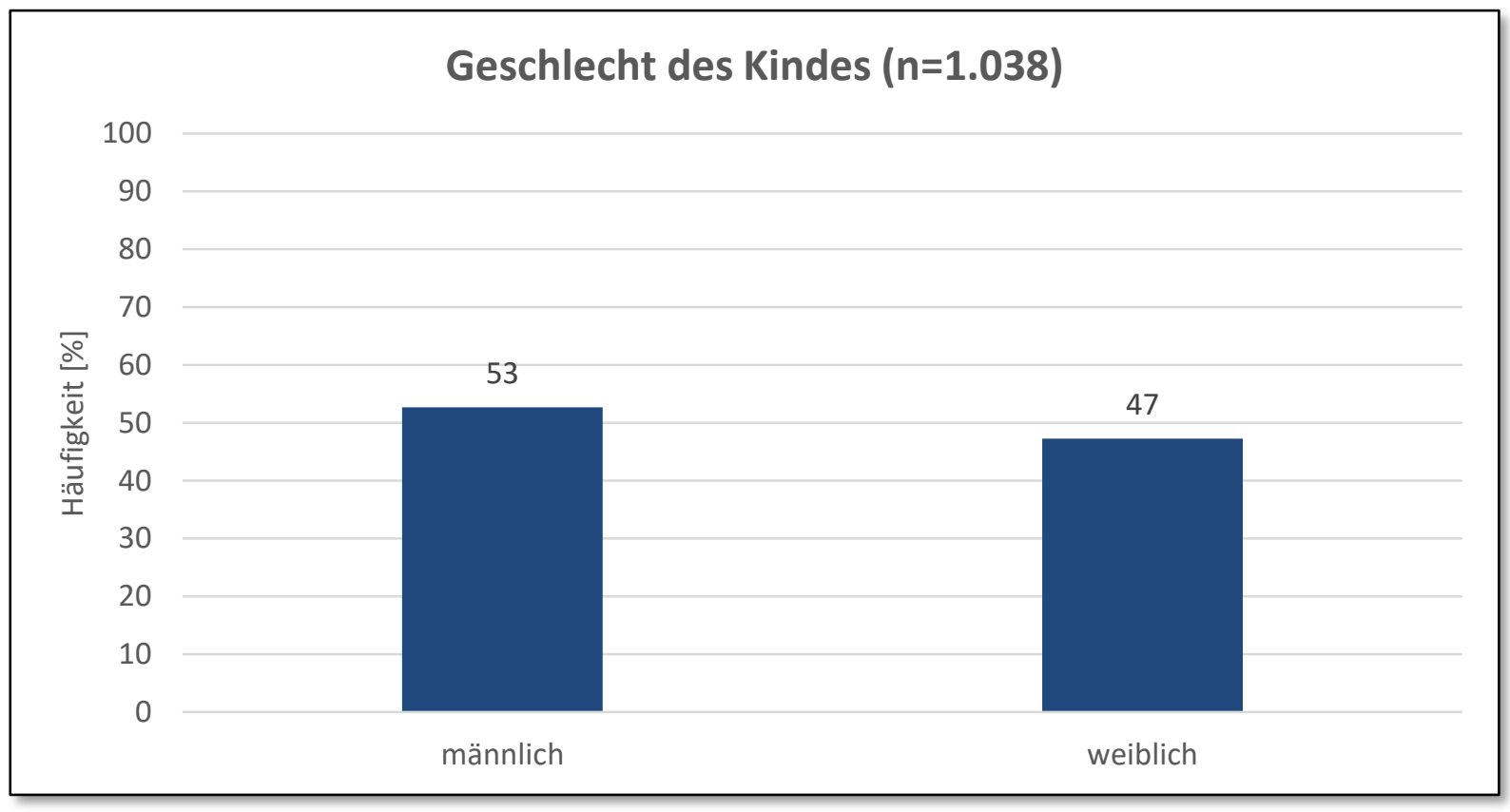

Abbildung 51: Geschlecht des Kindes 


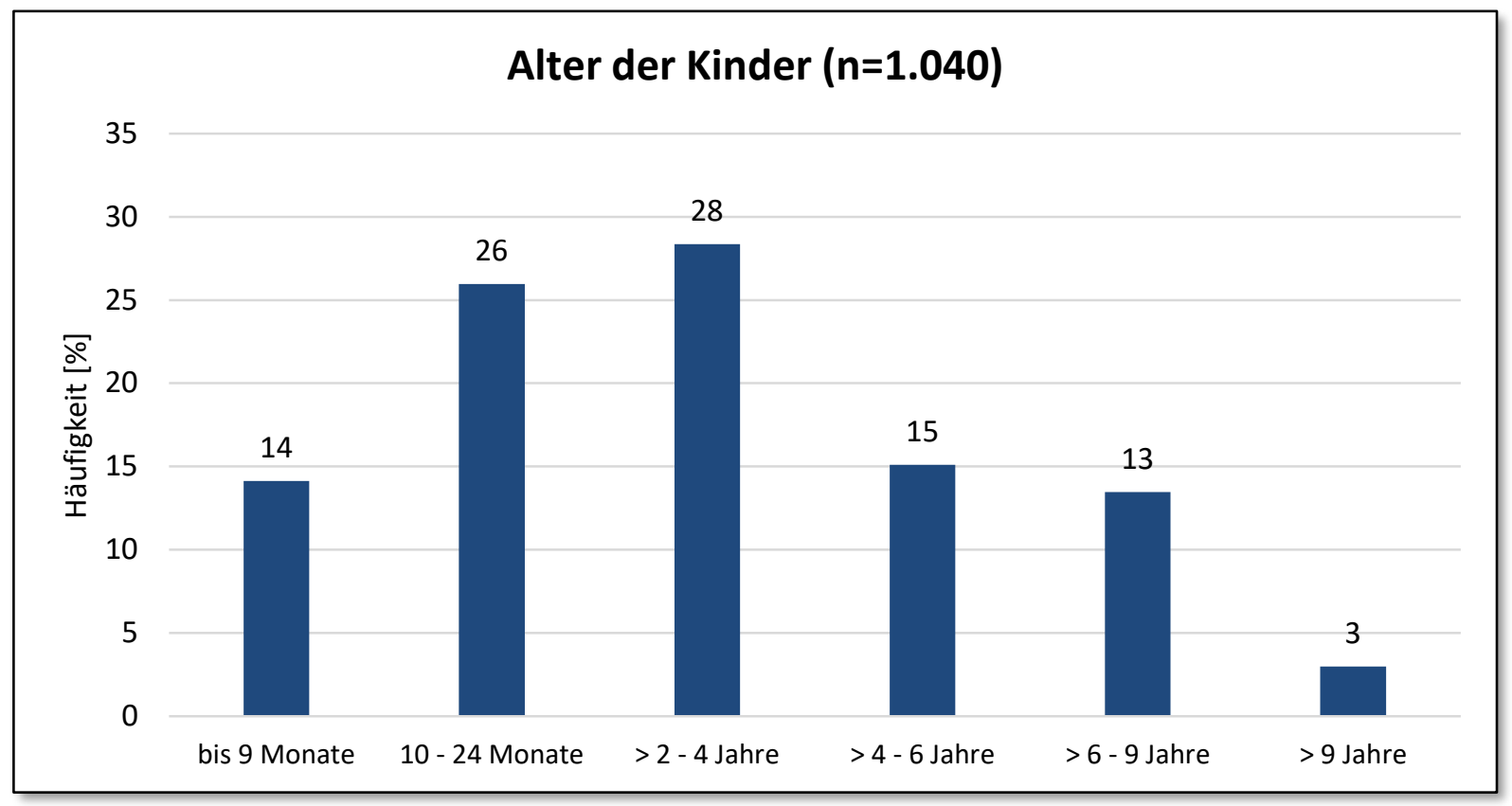

Abbildung 52: Alter der Kinder

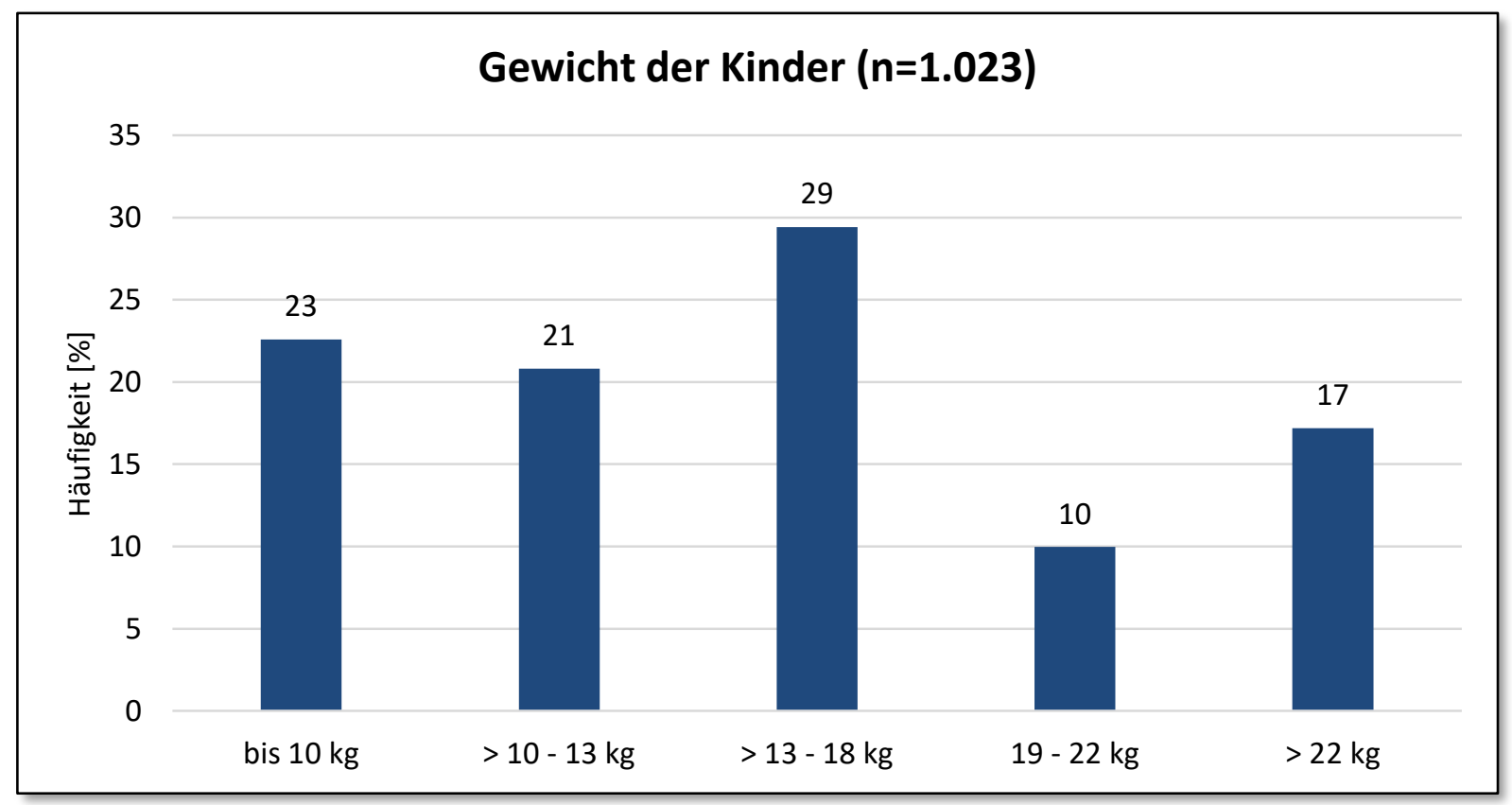

Abbildung 53: Gewicht der Kinder 


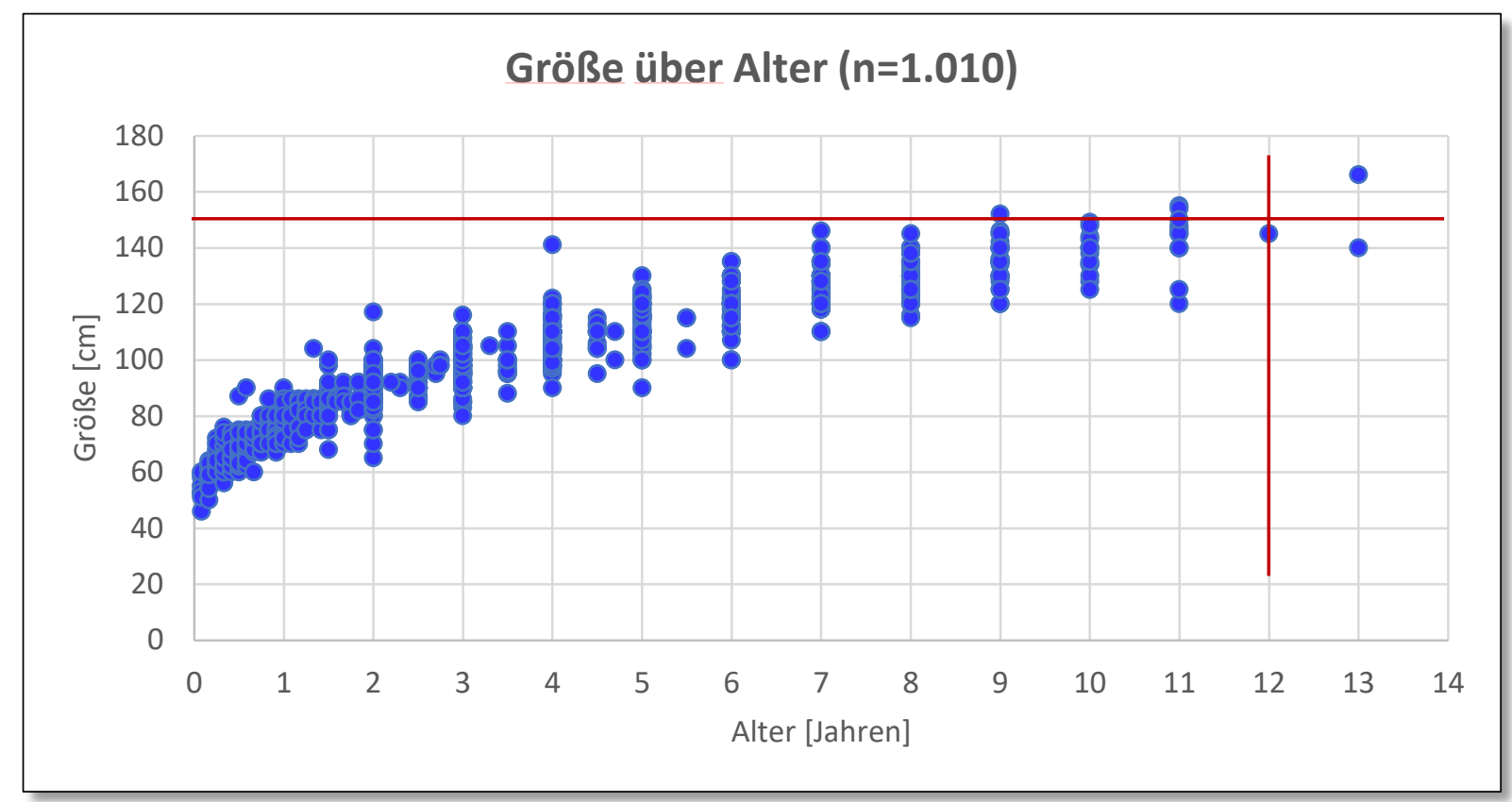

Abbildung 54: Größe über Alter der Kinder

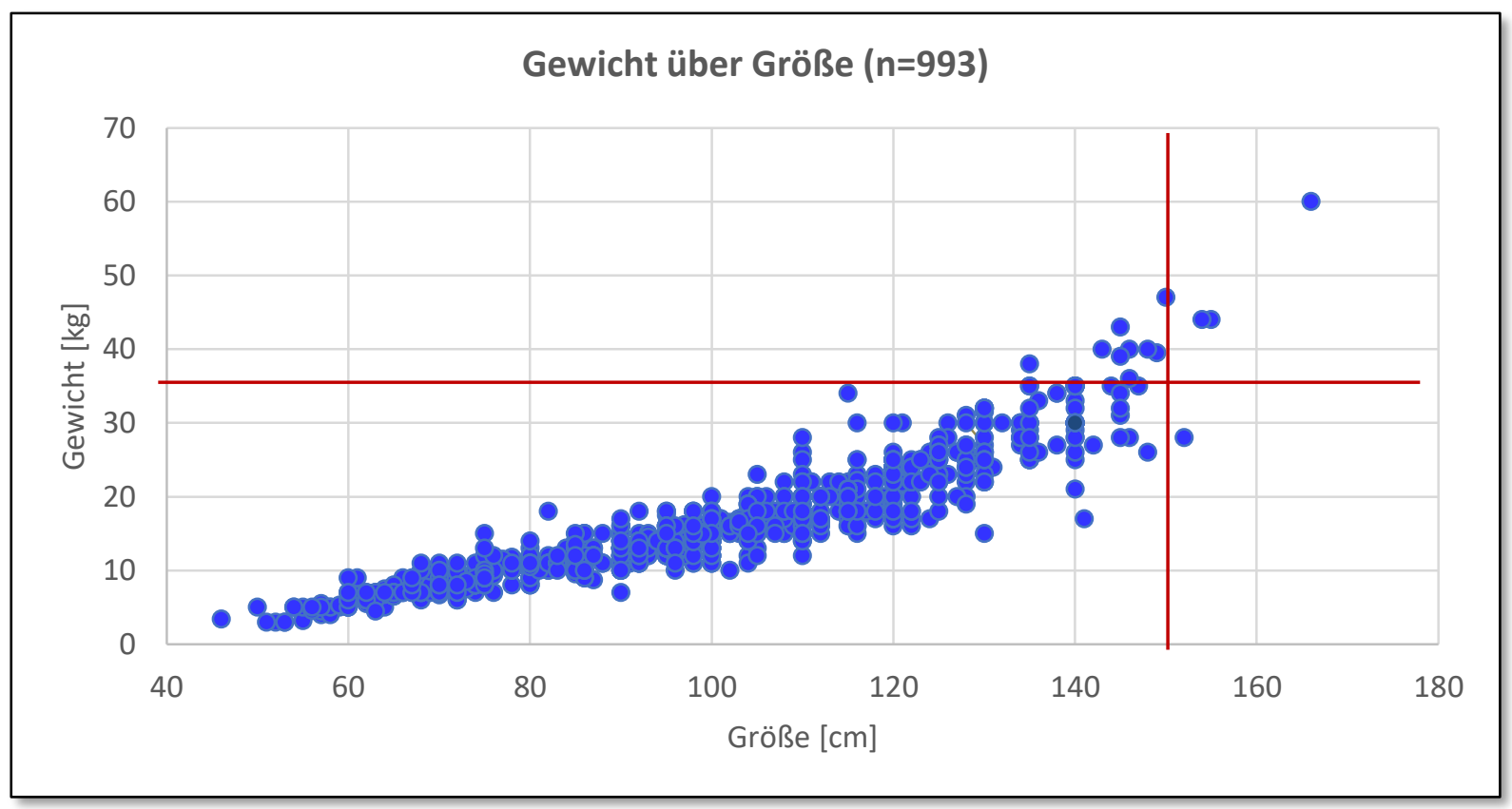

Abbildung 55: Gewicht über Größe der Kinder 


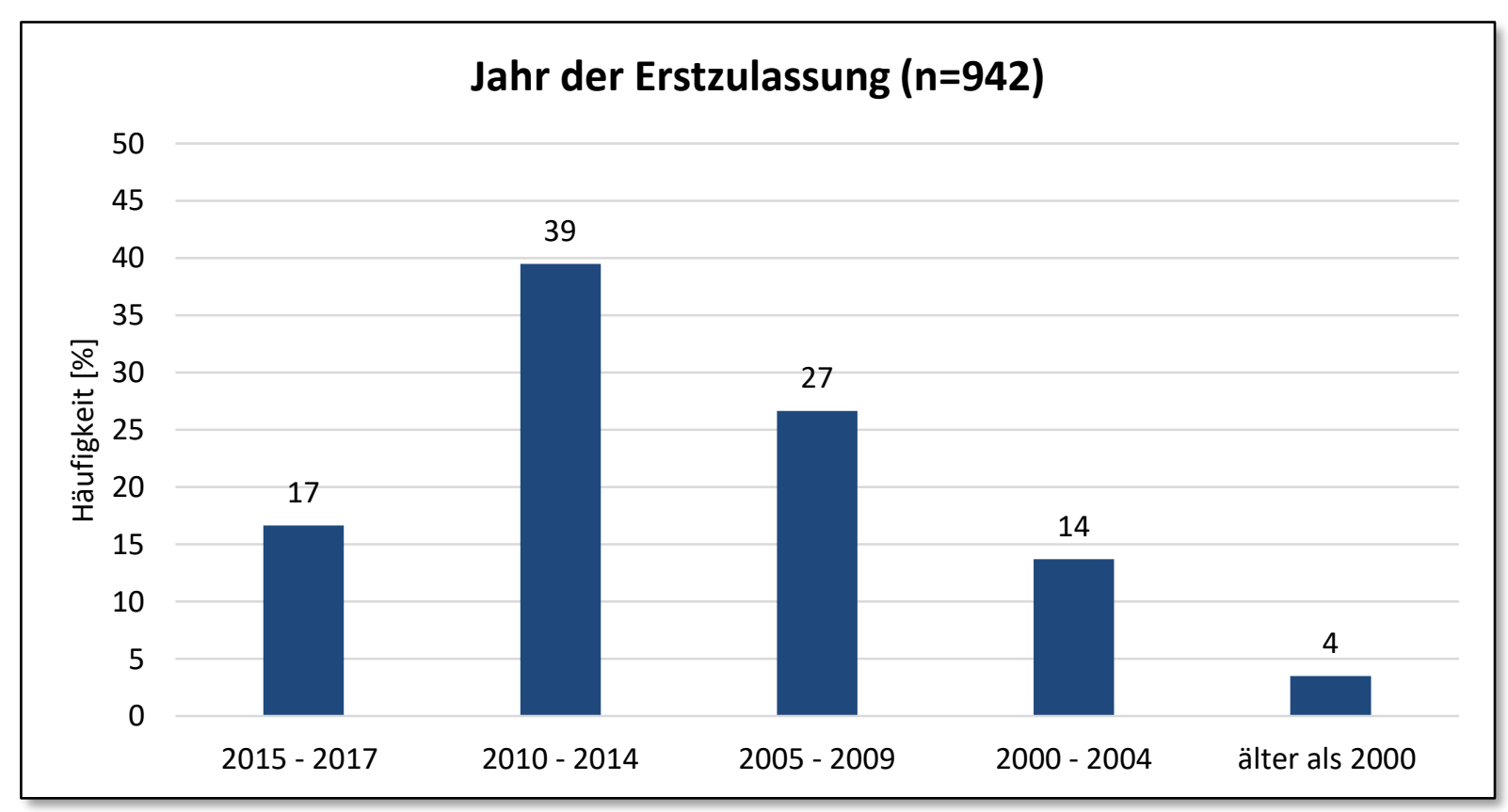

Abbildung 56: Jahr der Erstzulassung

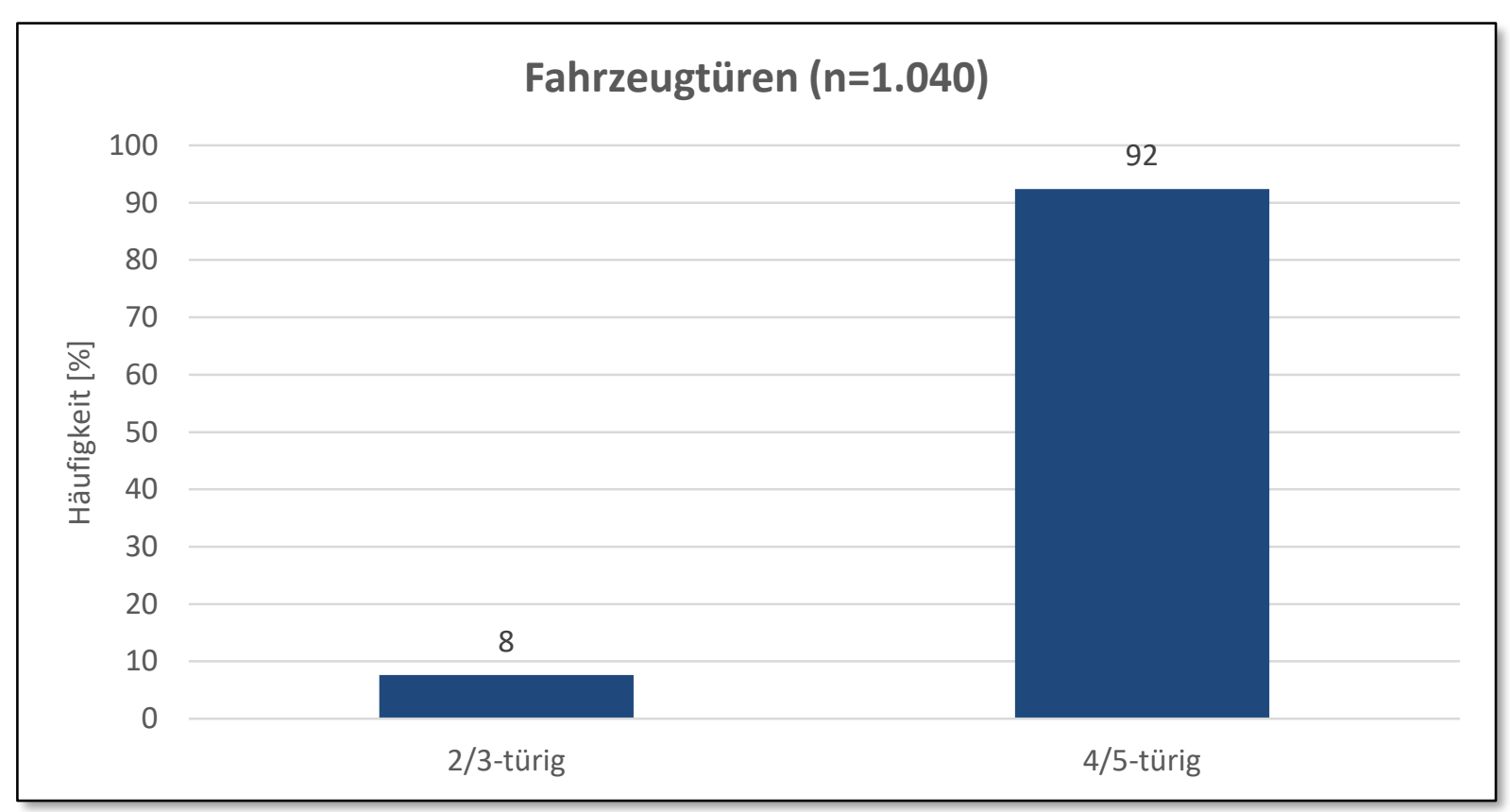

Abbildung 57: Fahrzeugtüren 


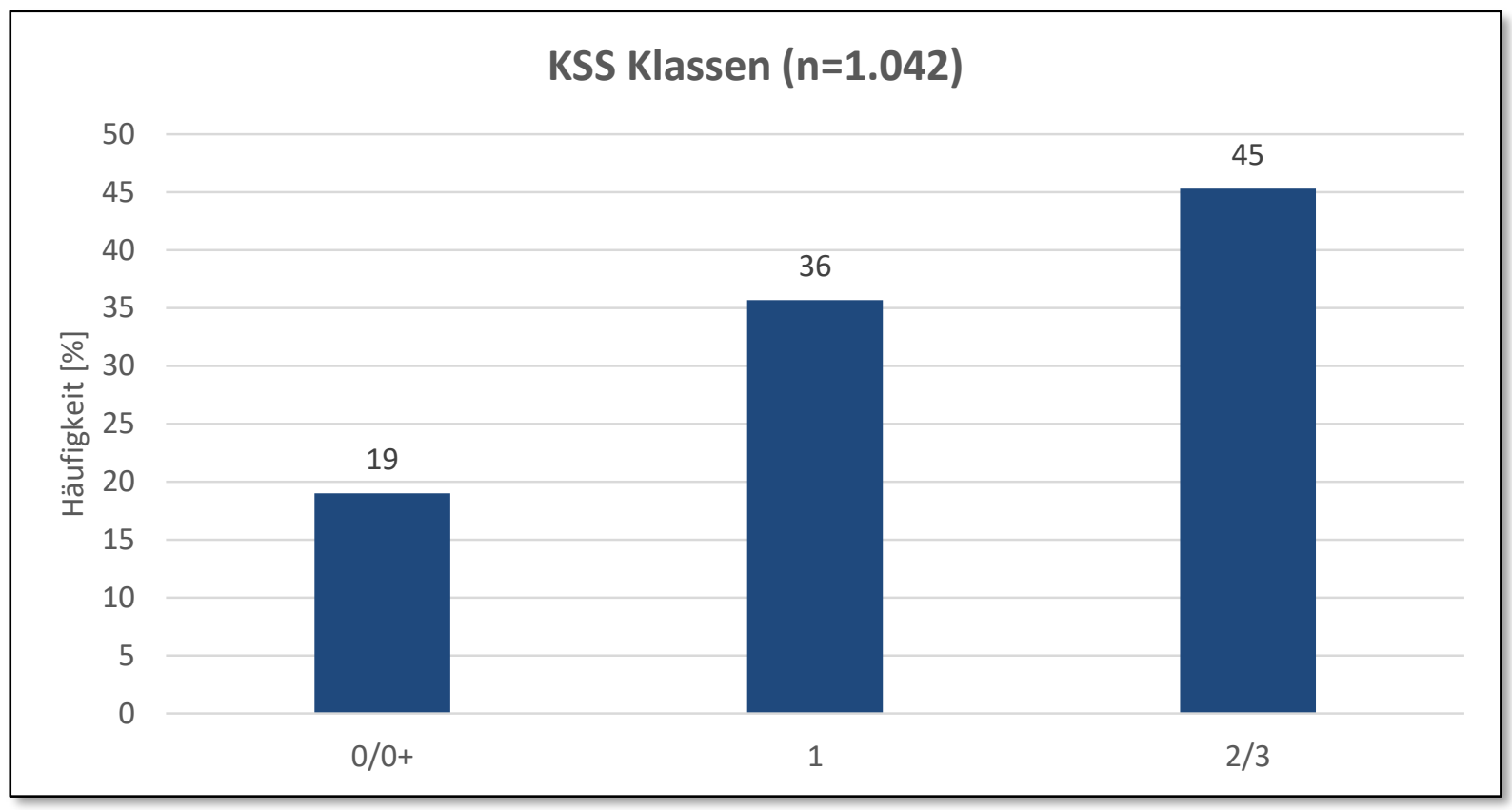

Abbildung 58: KSS-Klassen

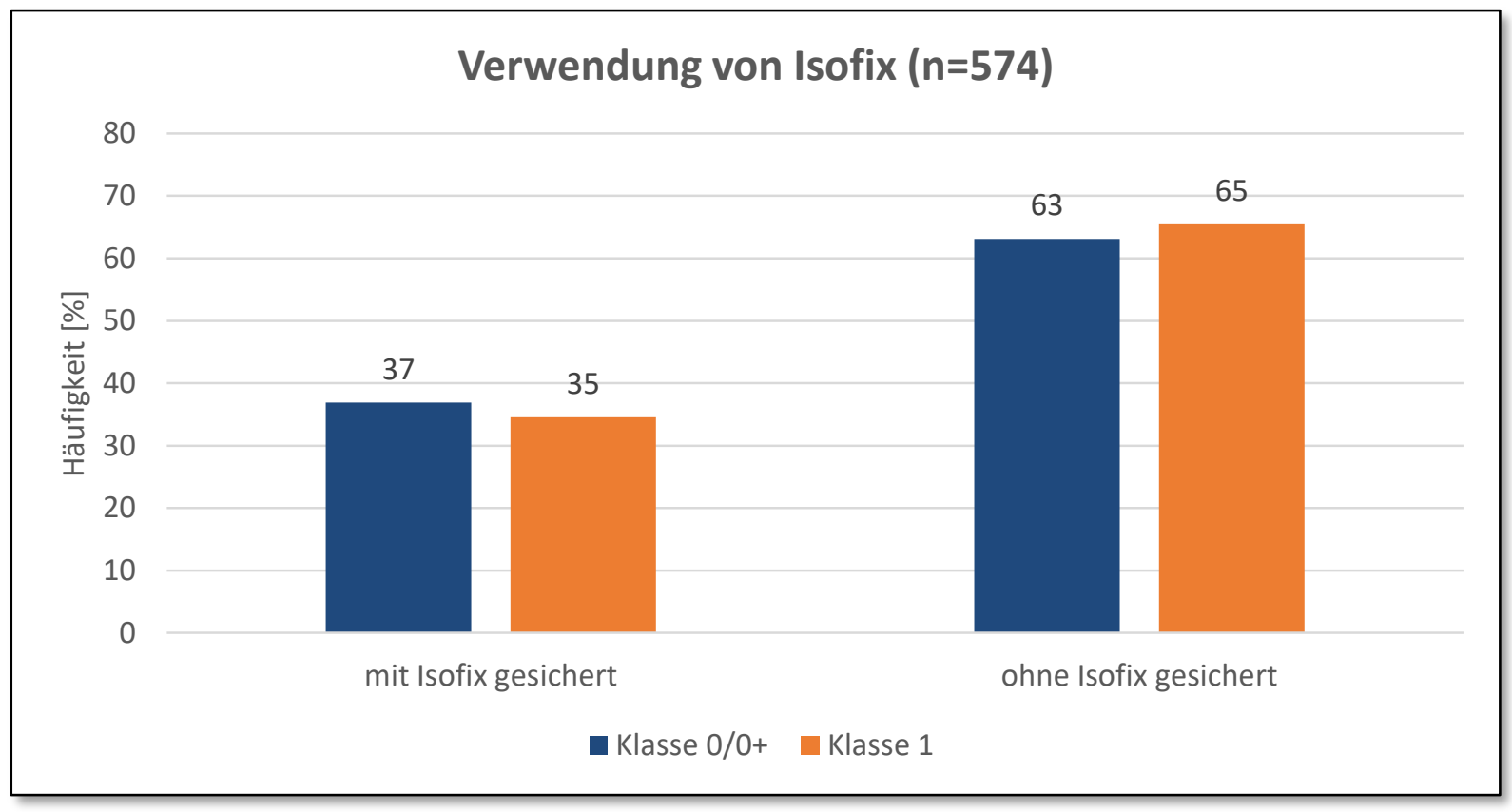

Abbildung 59: Verwendung von ISOFIX 


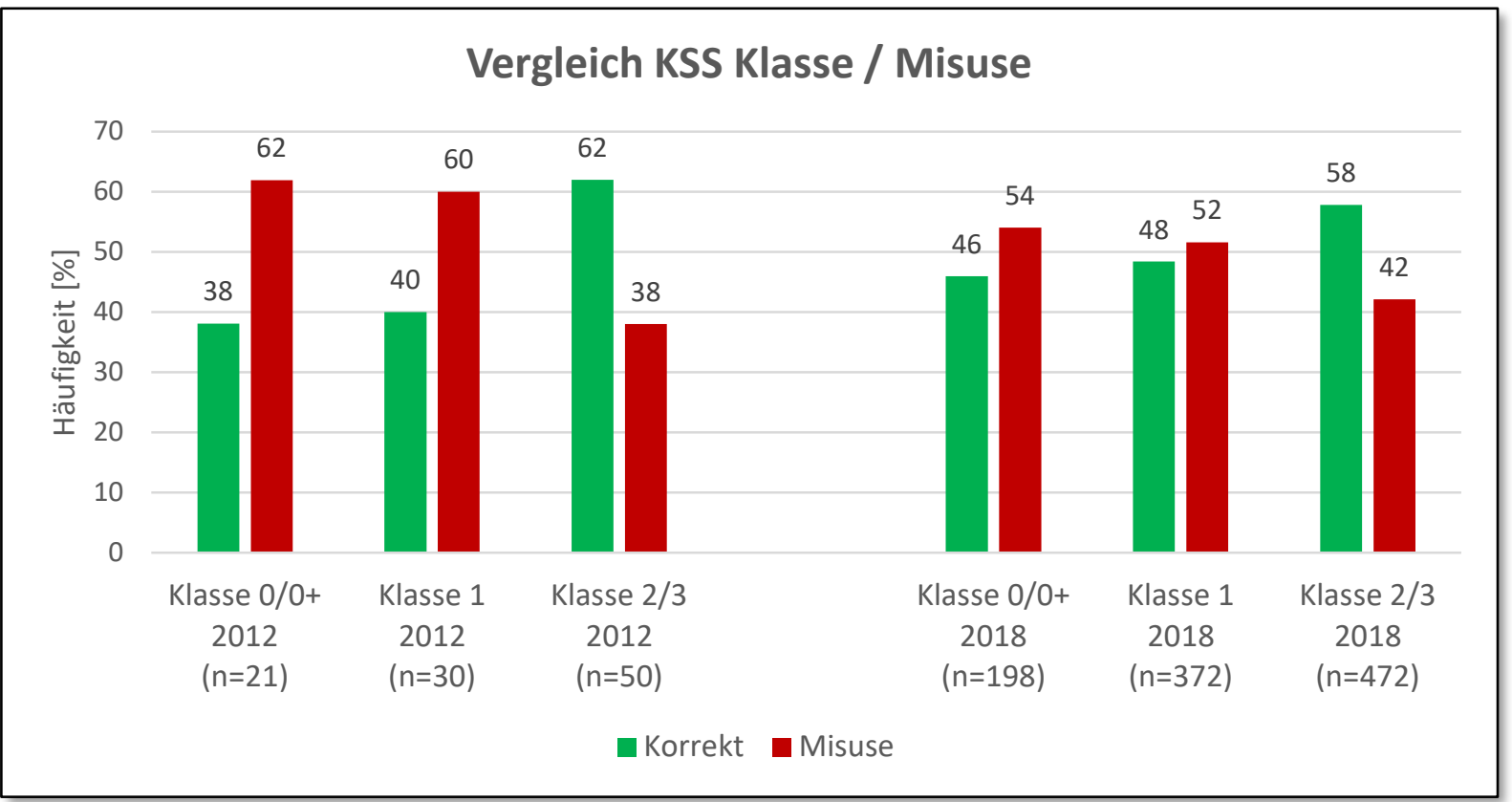

Abbildung 60: Vergleich KSS Klasse / Misuse

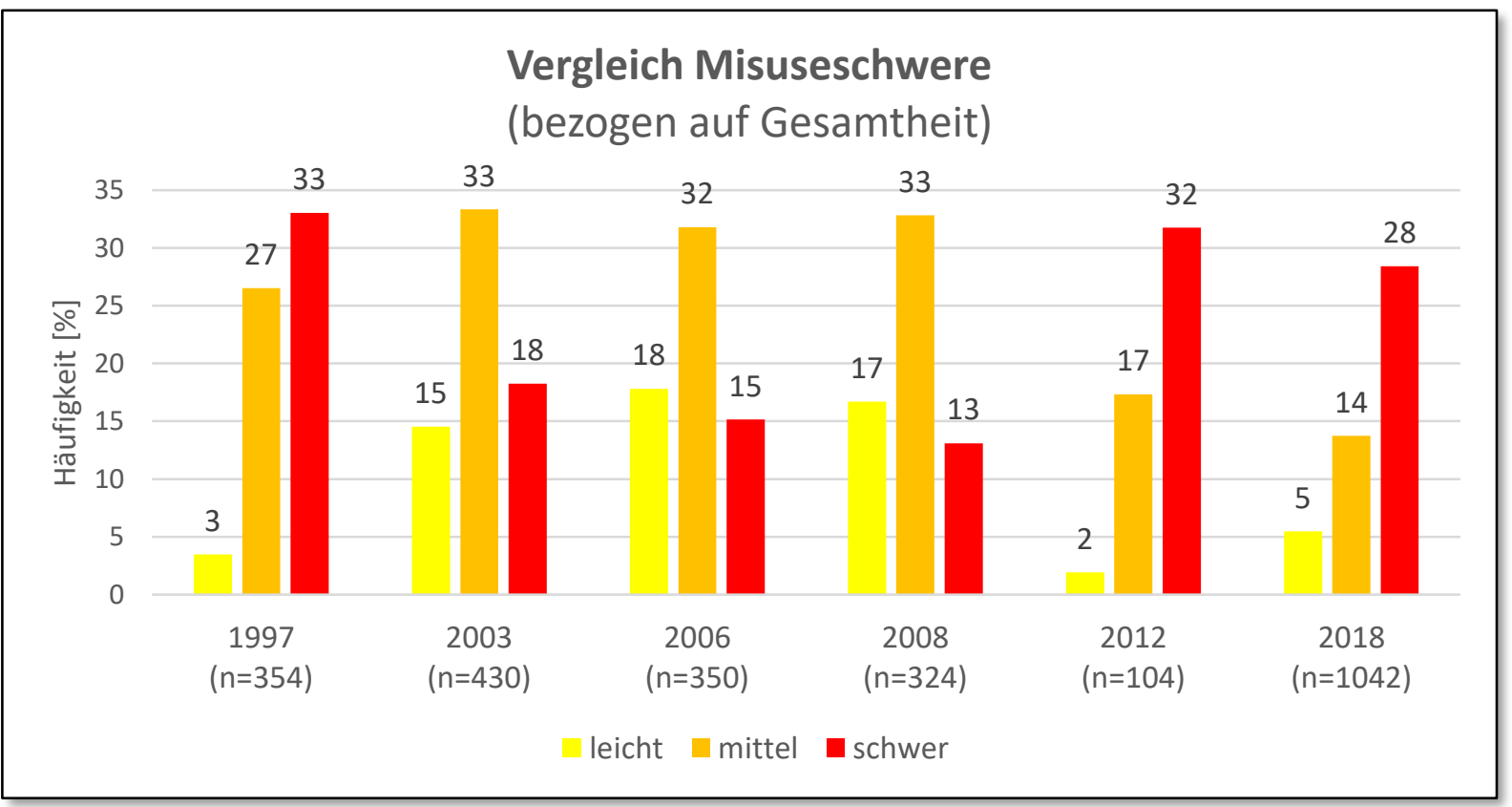

Abbildung 61: Vergleich Misuseschwere im Vergleich 


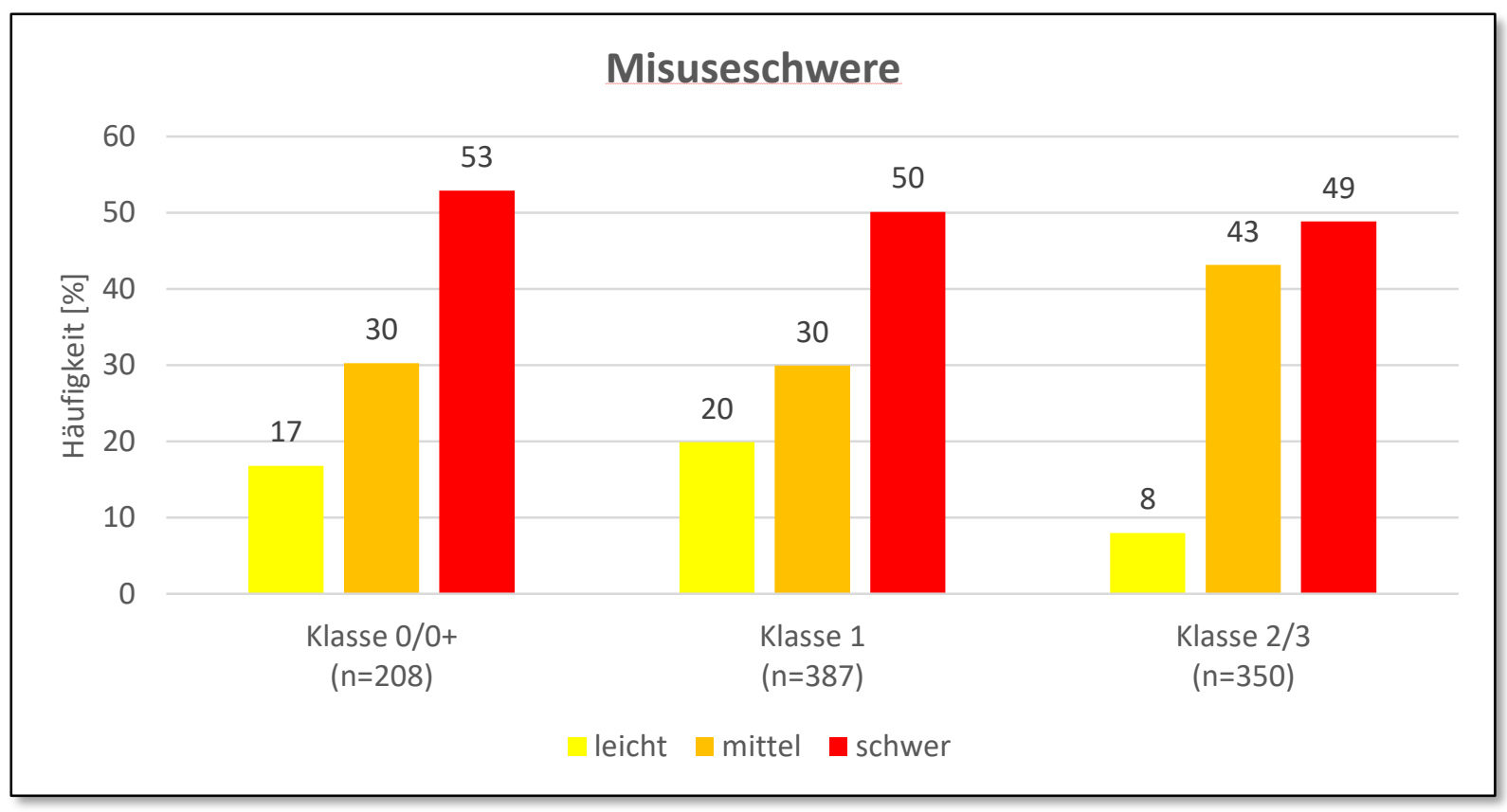

Abbildung 62: Misuseschwere

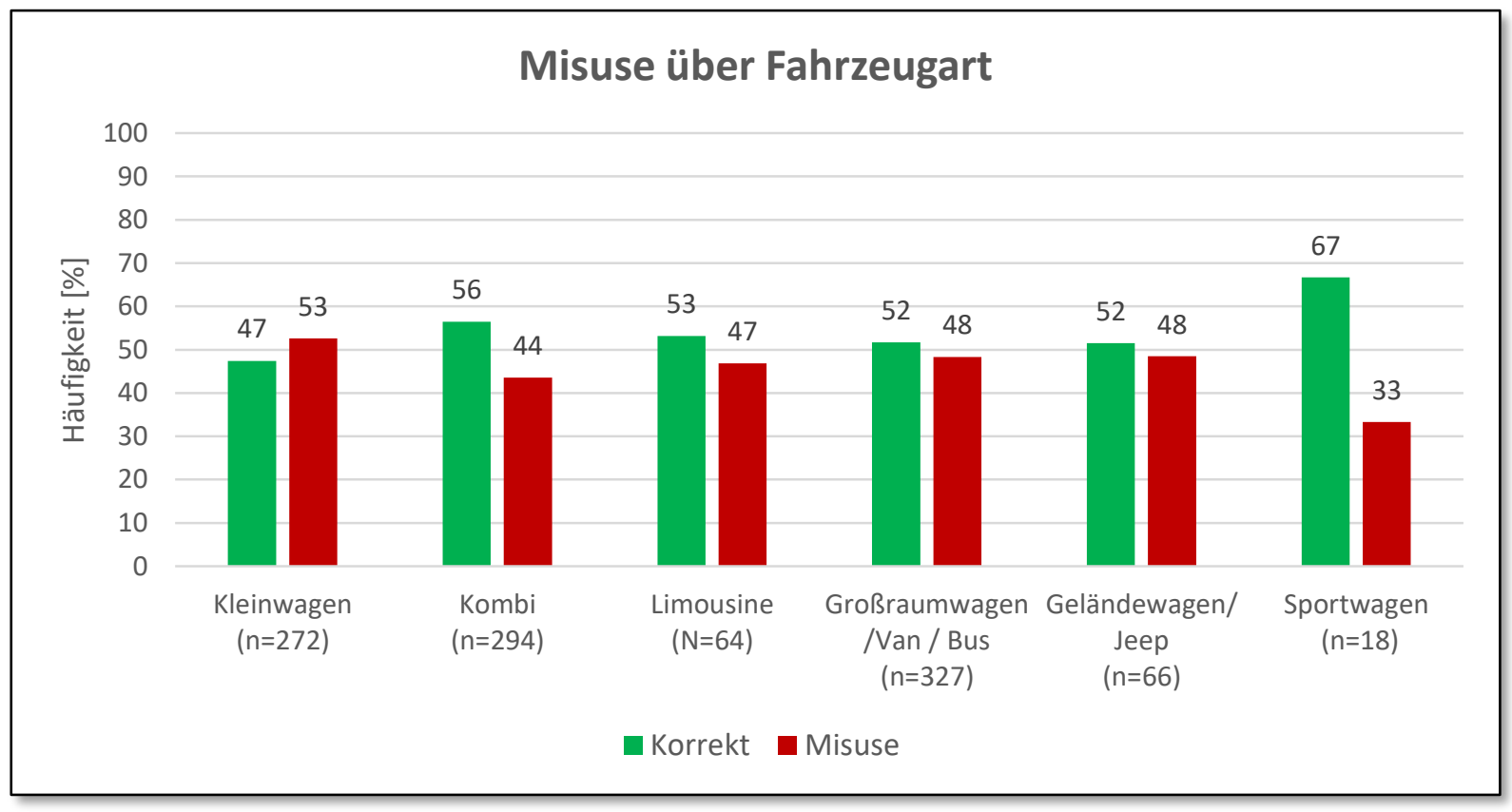

Abbildung 63: Misuse über Fahrzeugart 


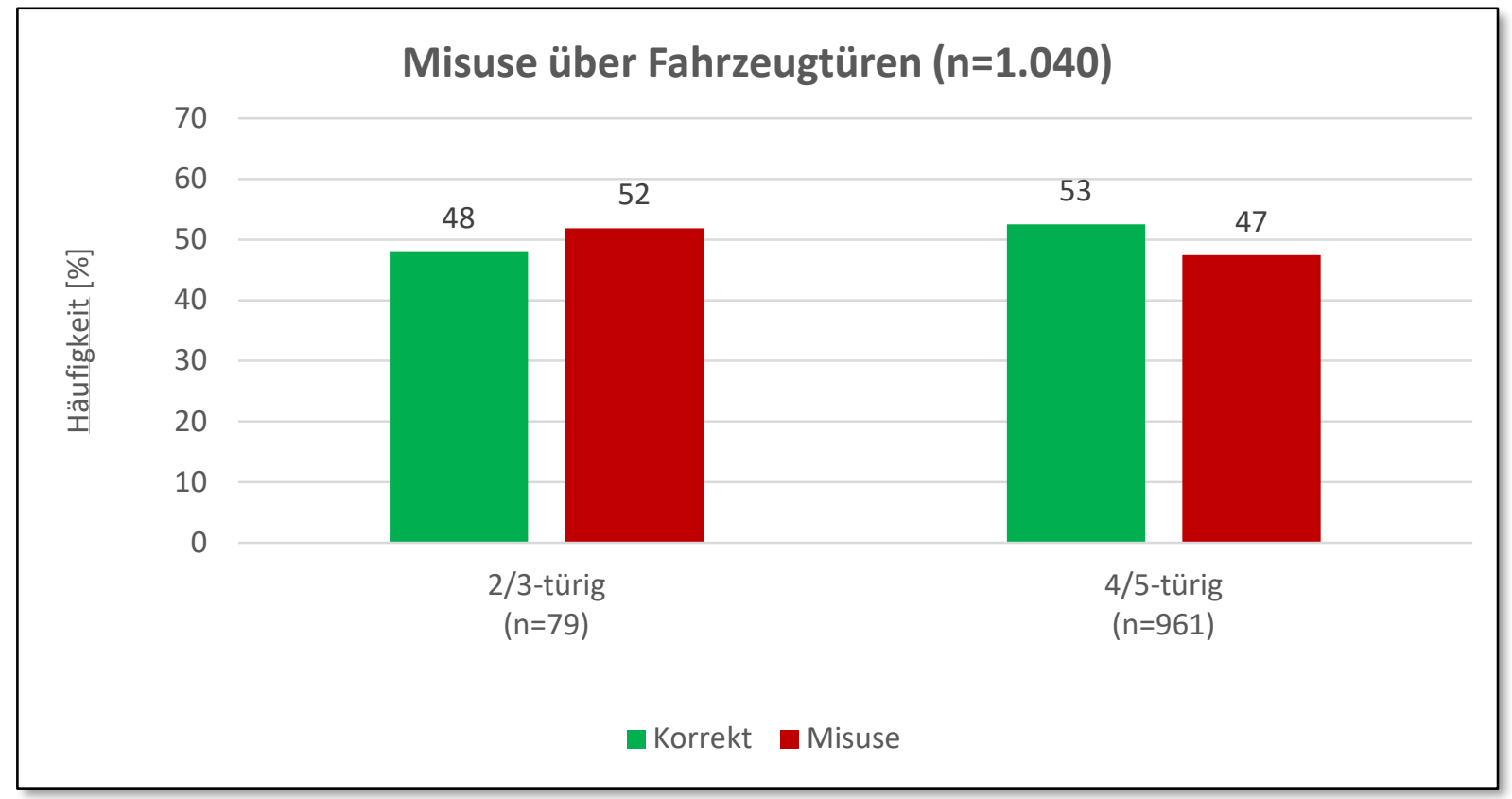

Abbildung 64: Misuse über Fahrzeugtüren

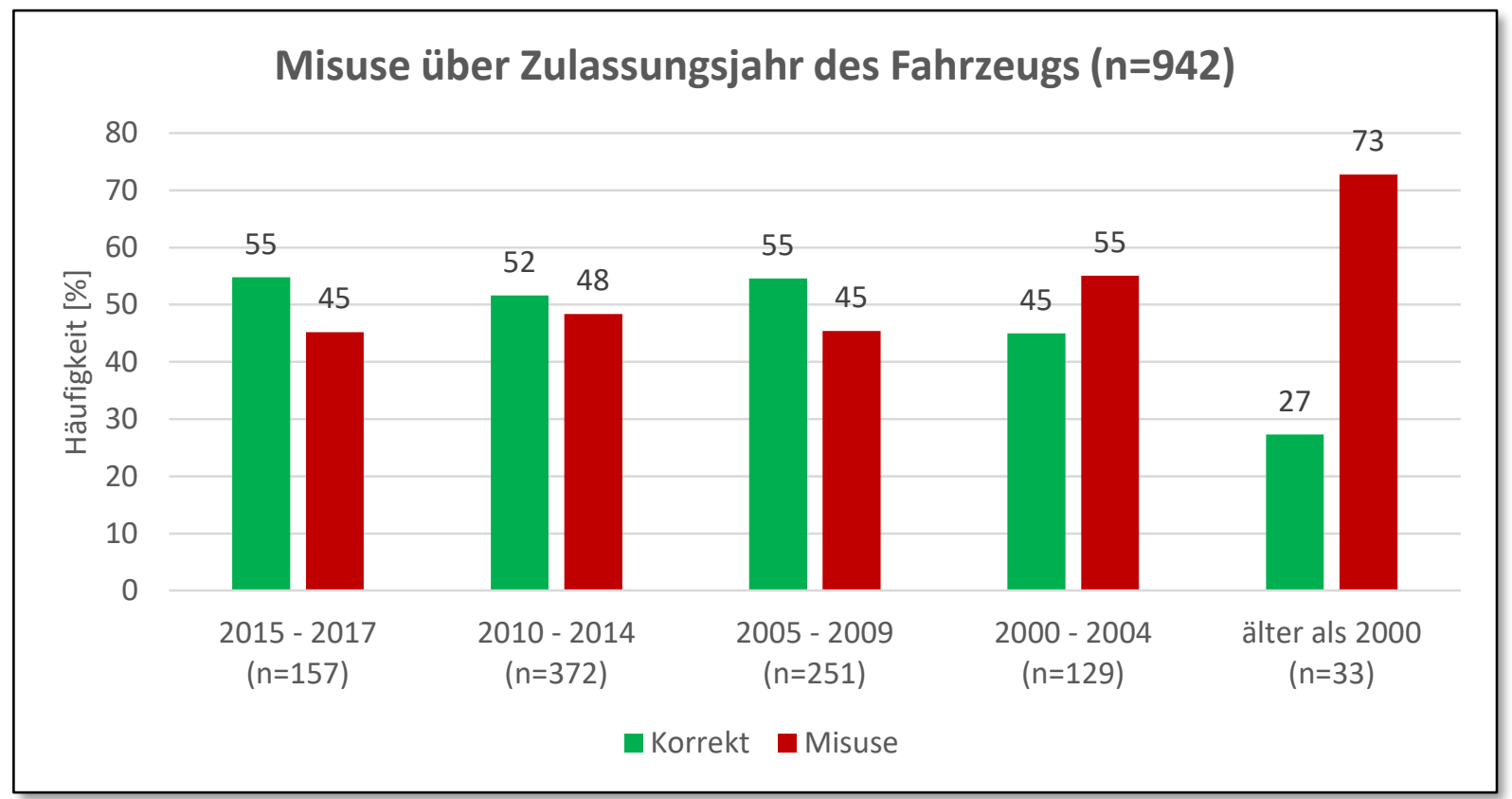

Abbildung 65: Misuse über Zulassungsjahr des Fahrzeuges 


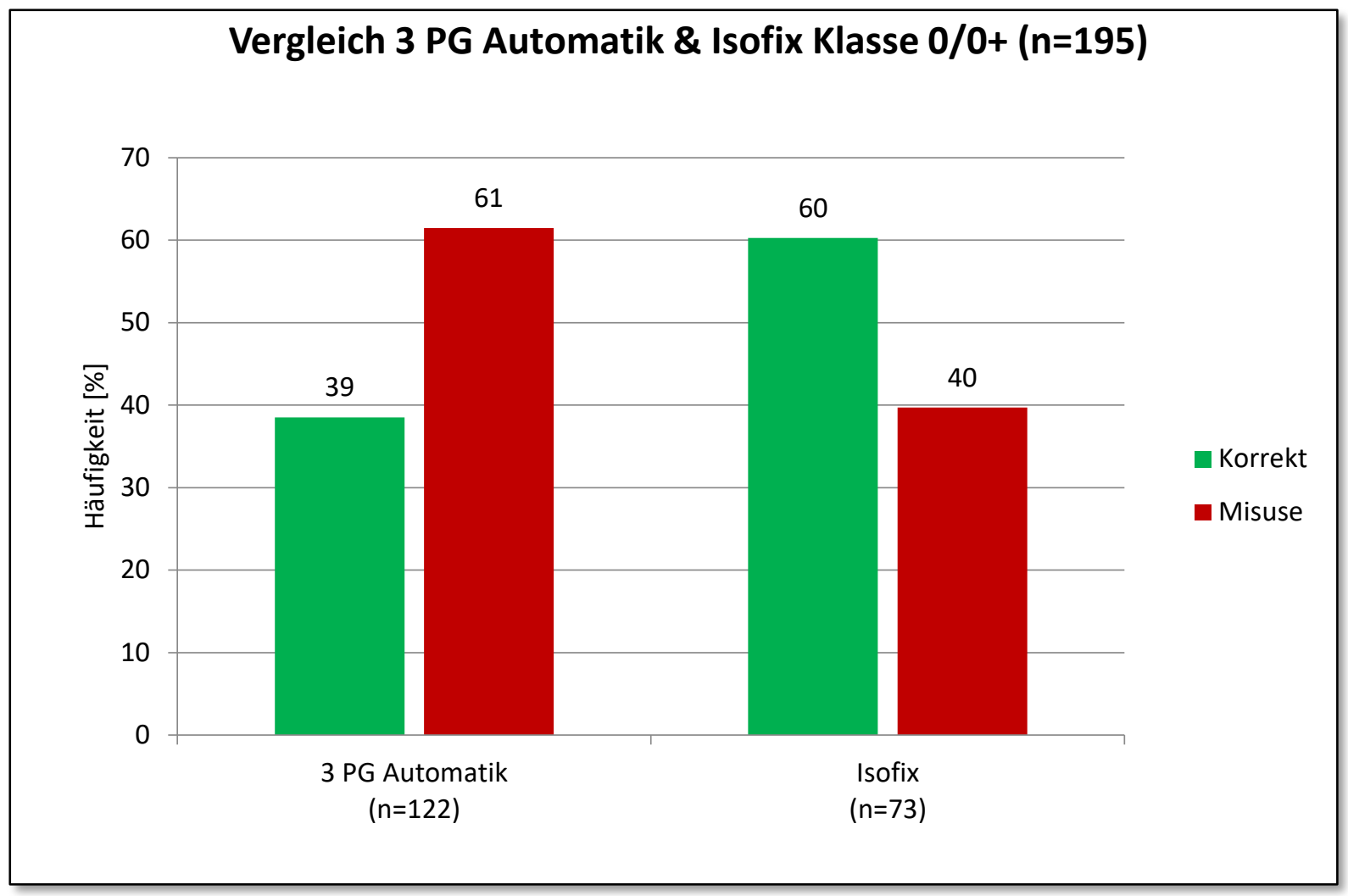

Abbildung 66: Vergleich 3PG und ISOFIX in KI.0/0+ 


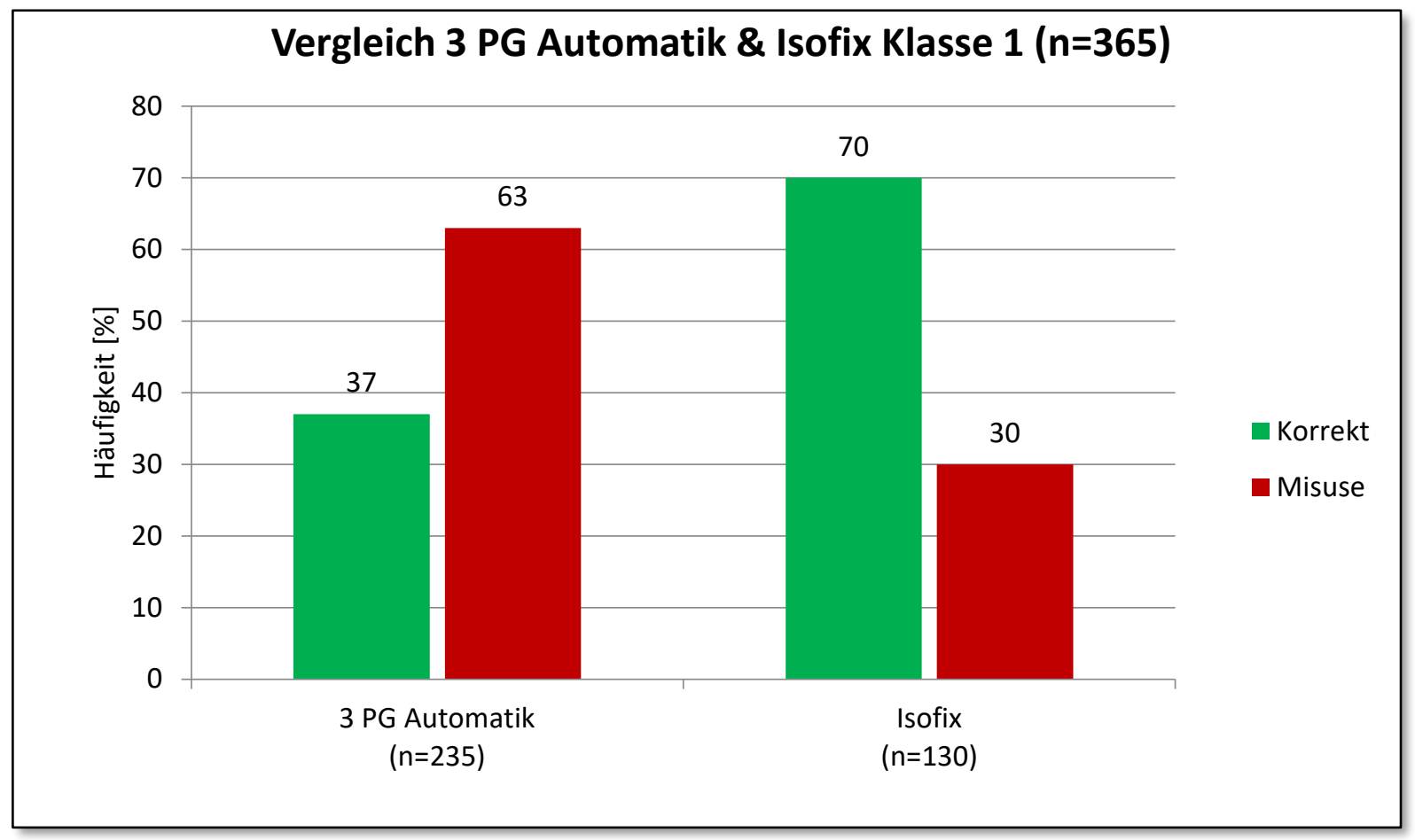

Abbildung 67: Vergleich 3PG und ISOFIX in KI.1

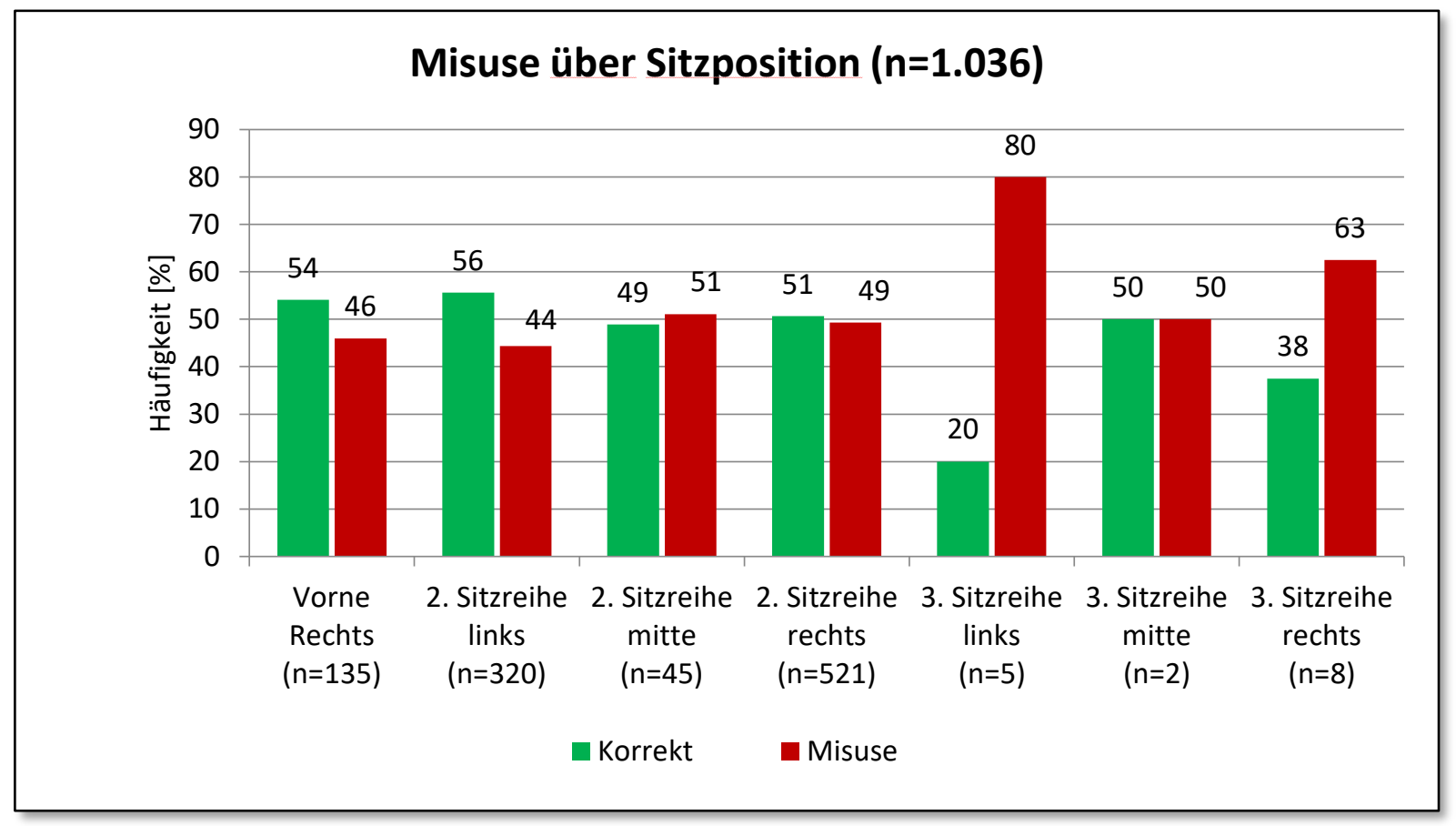

Abbildung 68: Misuse über Sitzposition 


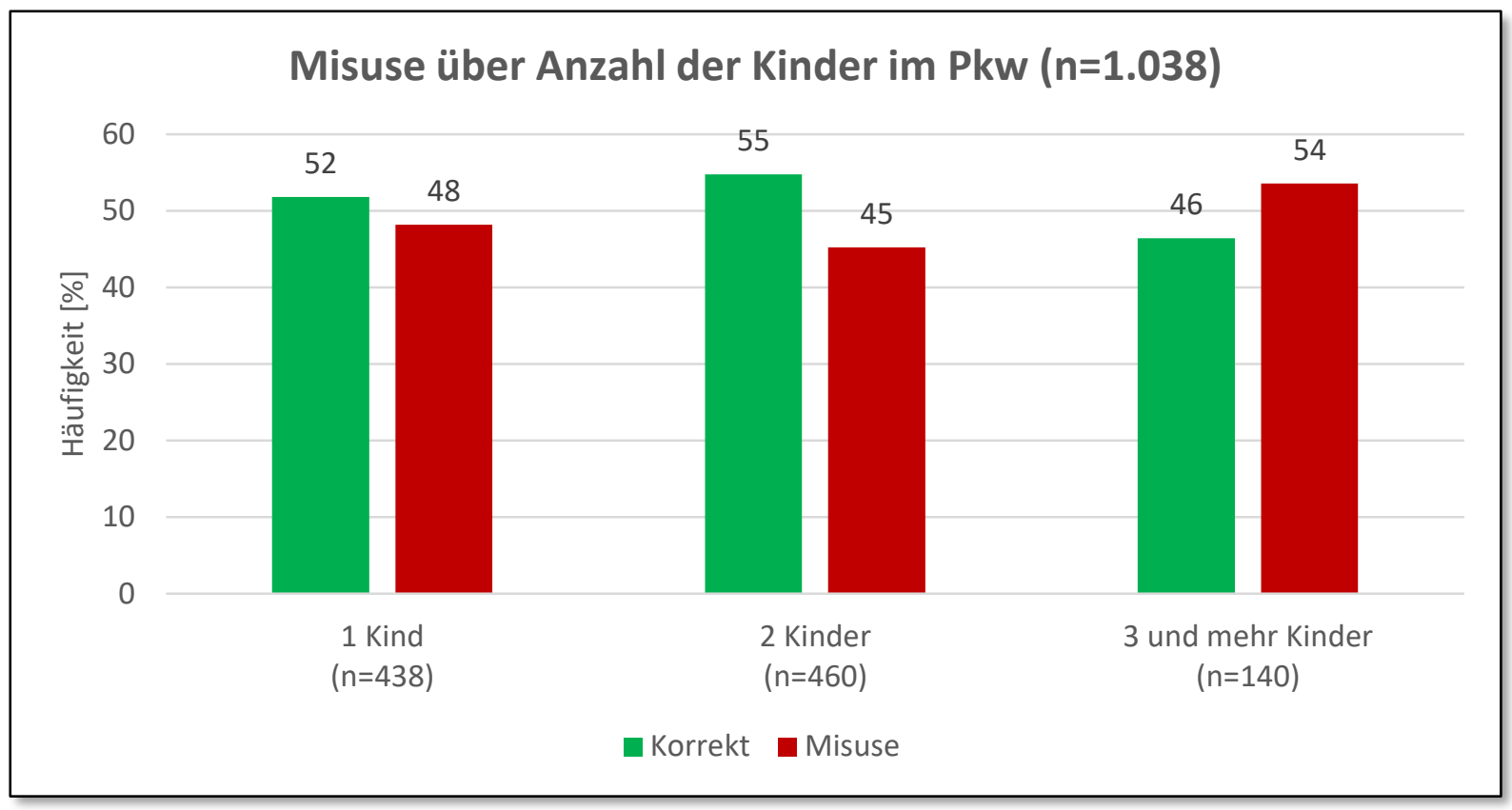

Abbildung 69: Misuse über Anzahl der Kinder im Pkw

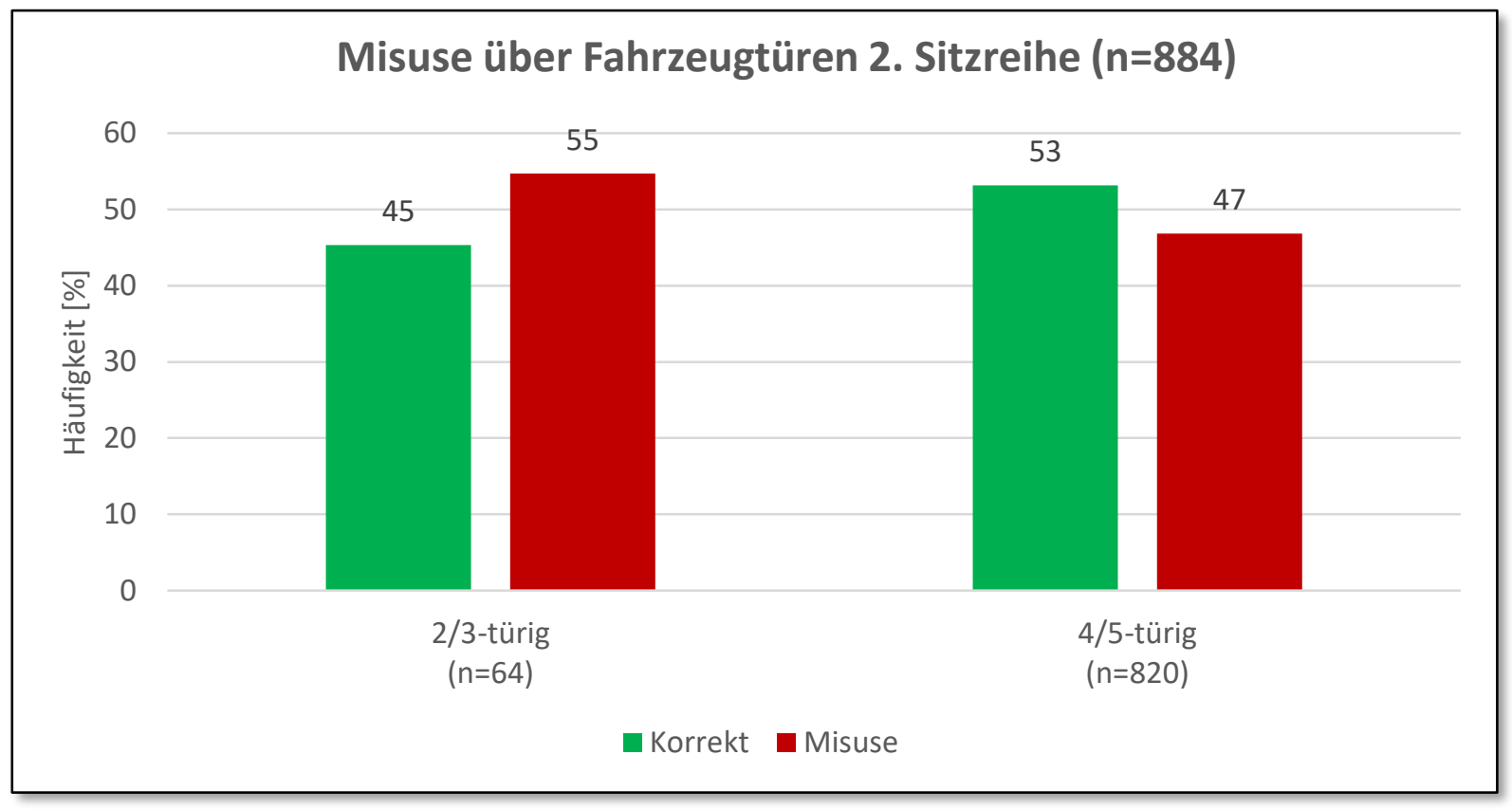

Abbildung 70: Misuseverteilung nach Anzahl an Fahrzeugtüren 


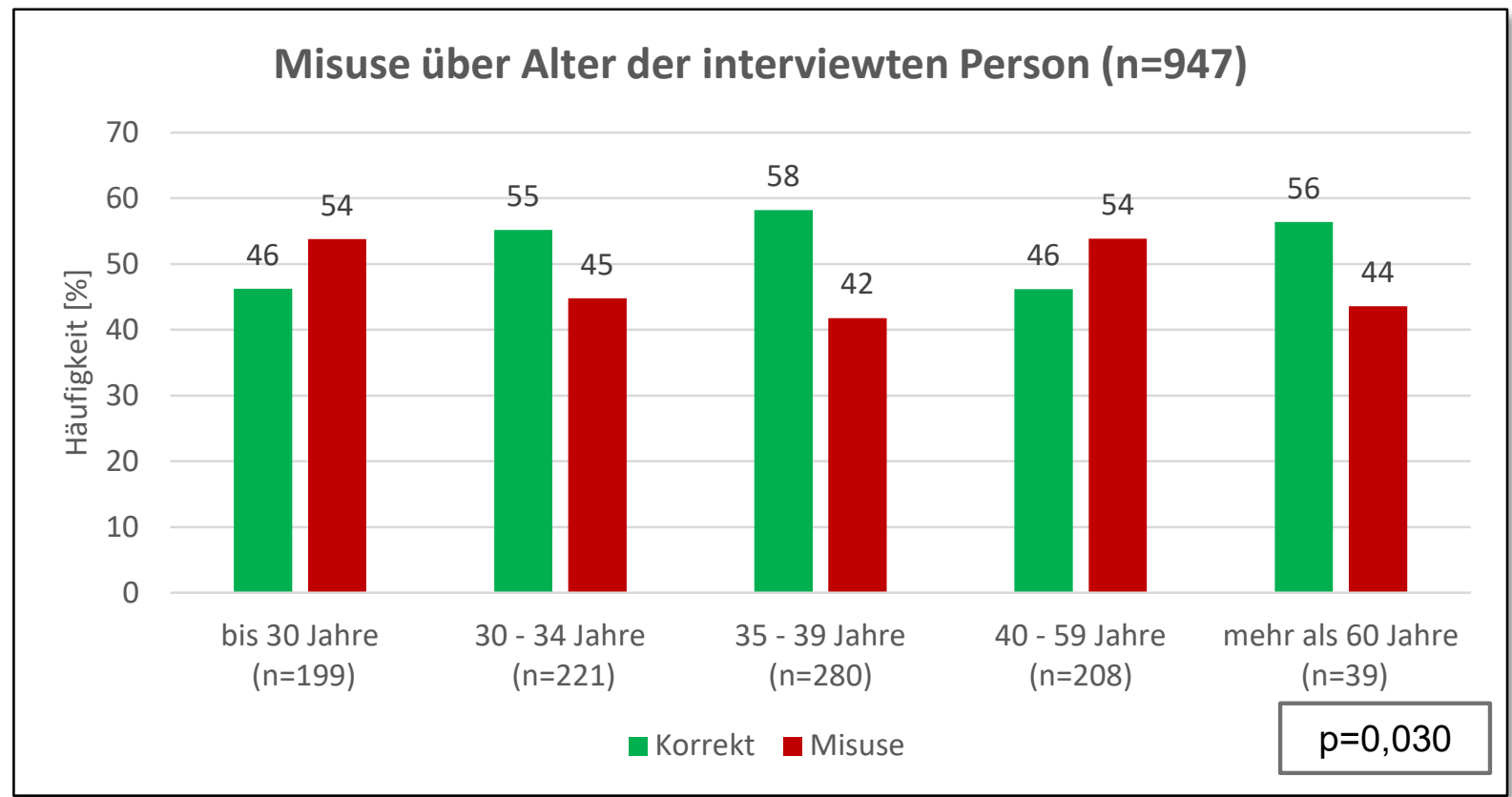

Abbildung 71: Misuse über Alter der interviewten Person

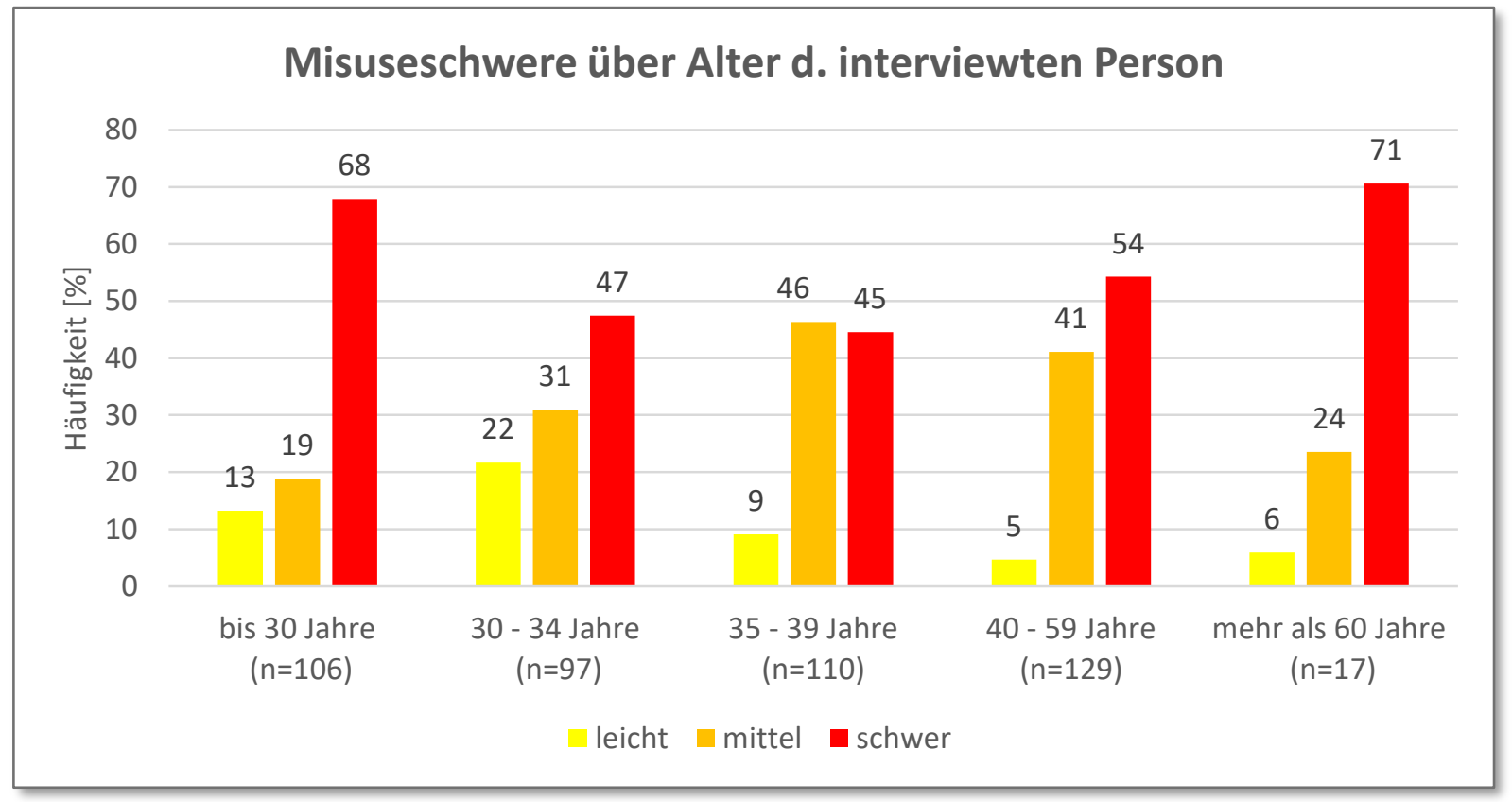

Abbildung 72: Misuseschwere über Alter der interviewten Person 


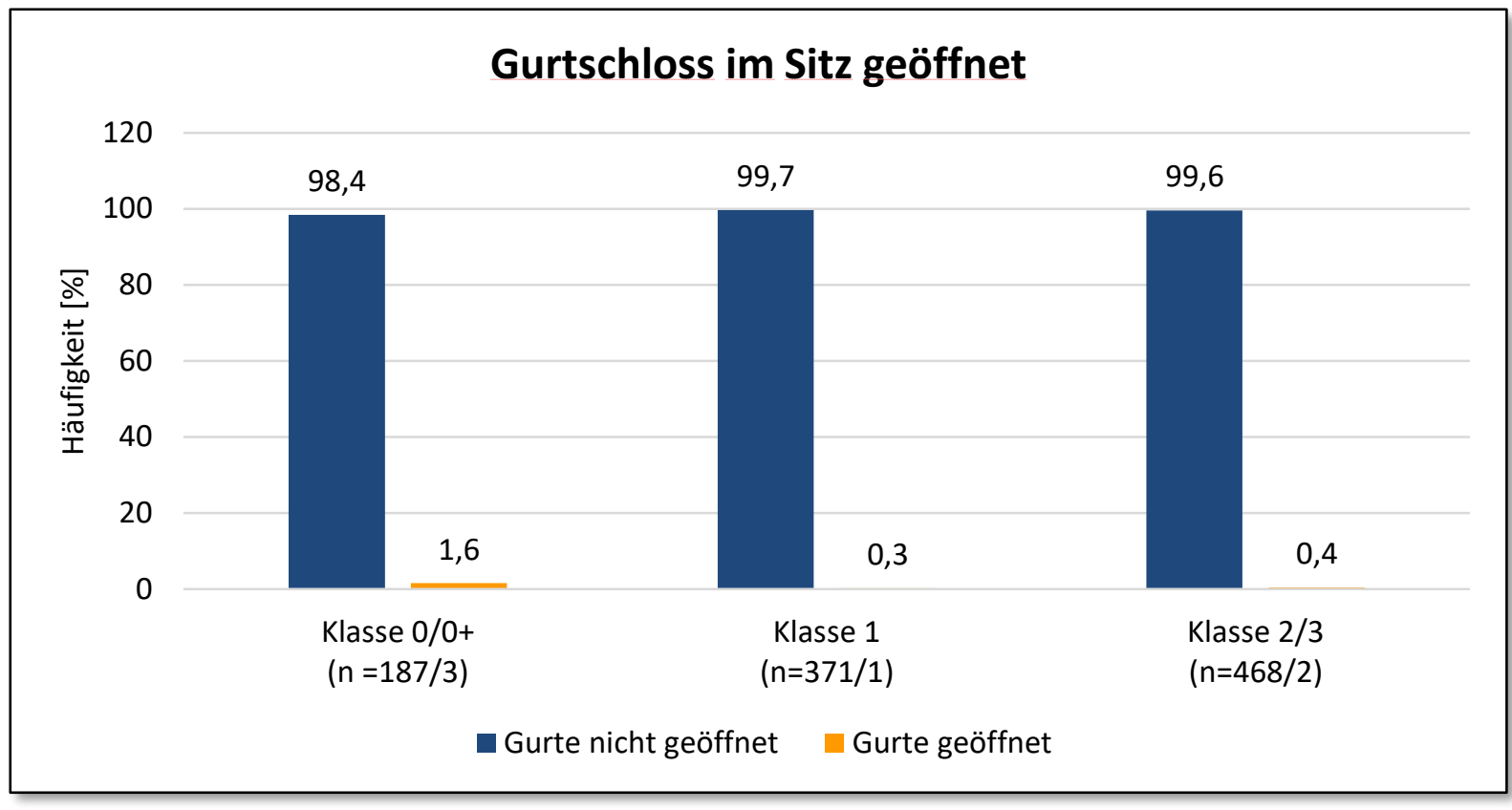

Abbildung 73: Gurtschloss im Sitz geöffnet

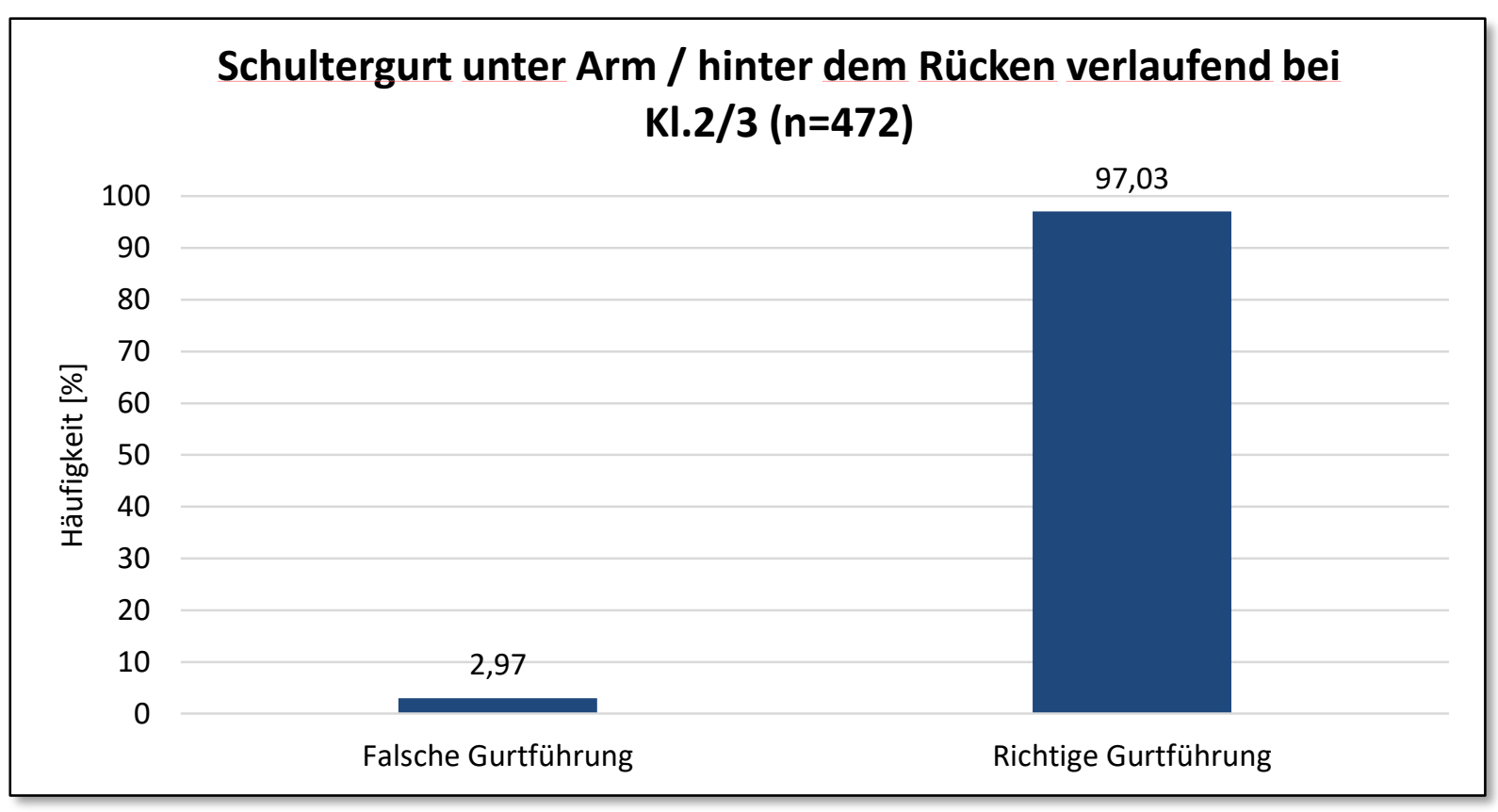

Abbildung 74: Schultergurt unter Arm / hinter dem Rücken verlaufend bei KI.2/3 


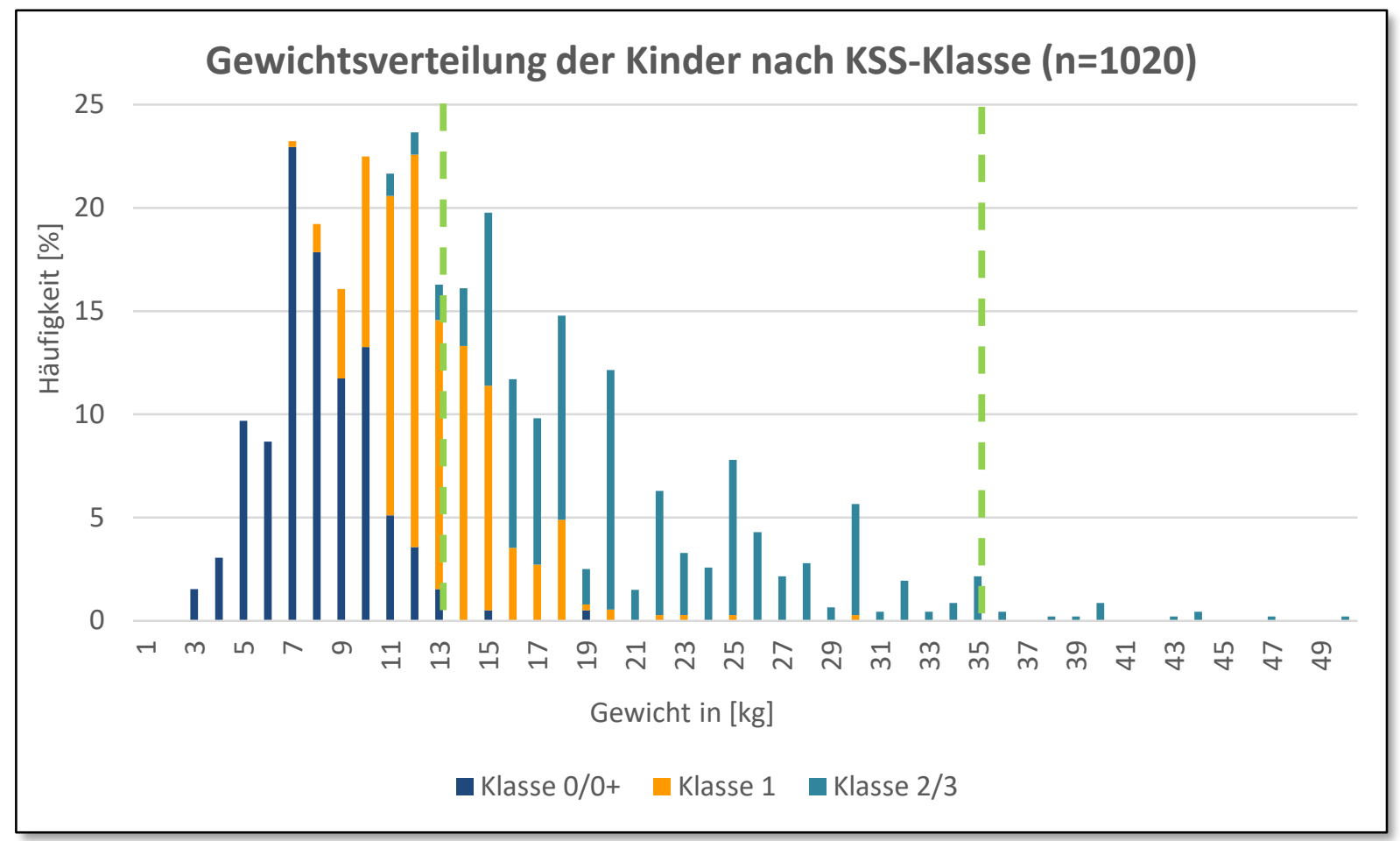

Abbildung 75: Gewichtsverteilung der Kinder nach KSS-Klasse

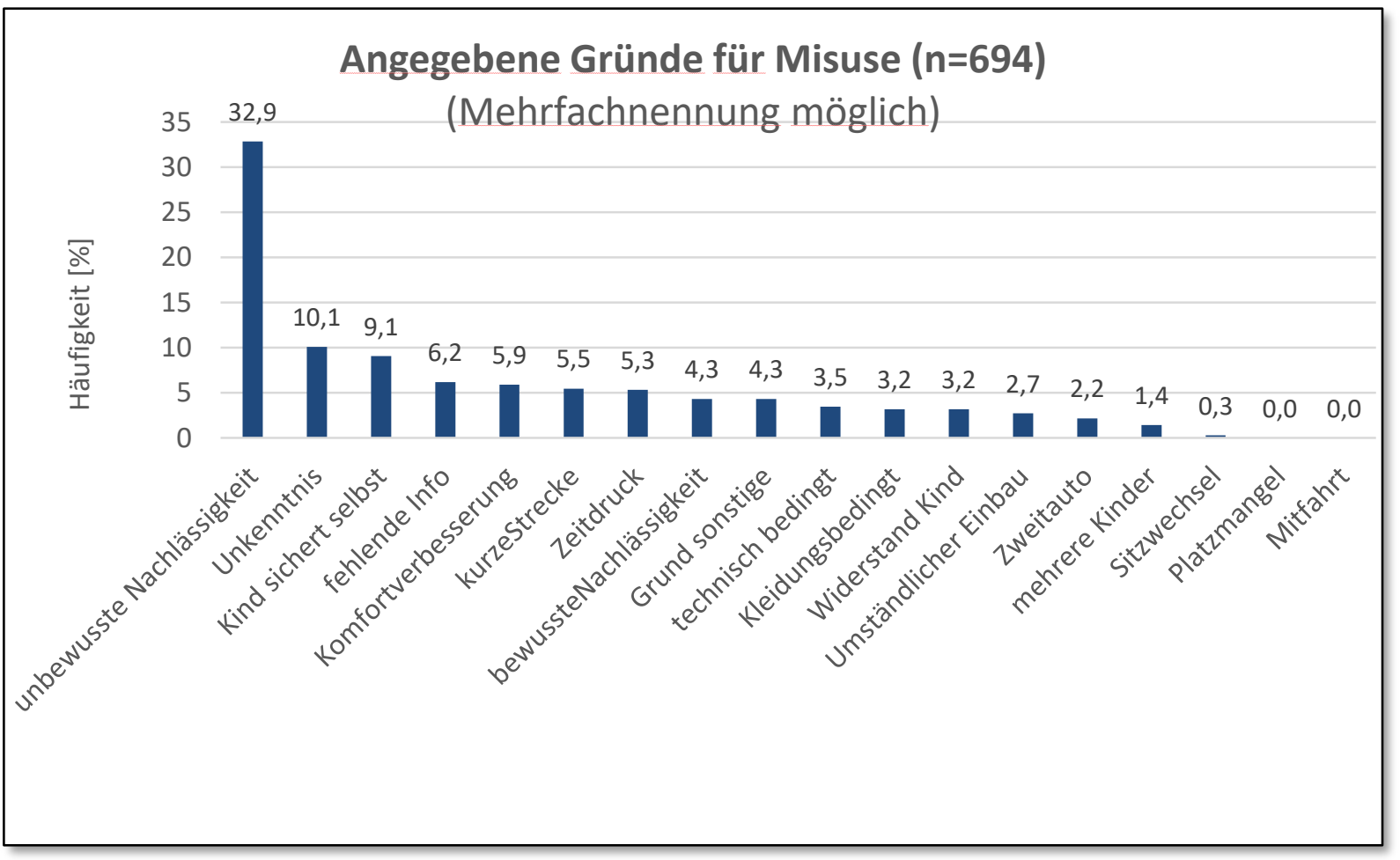

Abbildung 76: Angegebene Gründe für Misuse 


\subsection{Ergebnisse der Schlittenversuche zur Darstellung der Folgen von Misuse}

Sowohl in der aktuellen als auch in den vorangegangenen Studien ist bei der Sicherung von Babyschalen (Klasse 0/0+-Sitze) ein Fehler immer wieder aufgefallen: das Vertauschen von Schulter- und Beckengurt. Dabei wird der Schultergurtabschnitt des Fahrzeuggurts nicht wie vorgesehen hinter der Babyschale geführt, sondern über der Babyschale. Entsprechend wird der Beckengurtabschnitt hinter der Schale geführt (s. Abbildung 77).

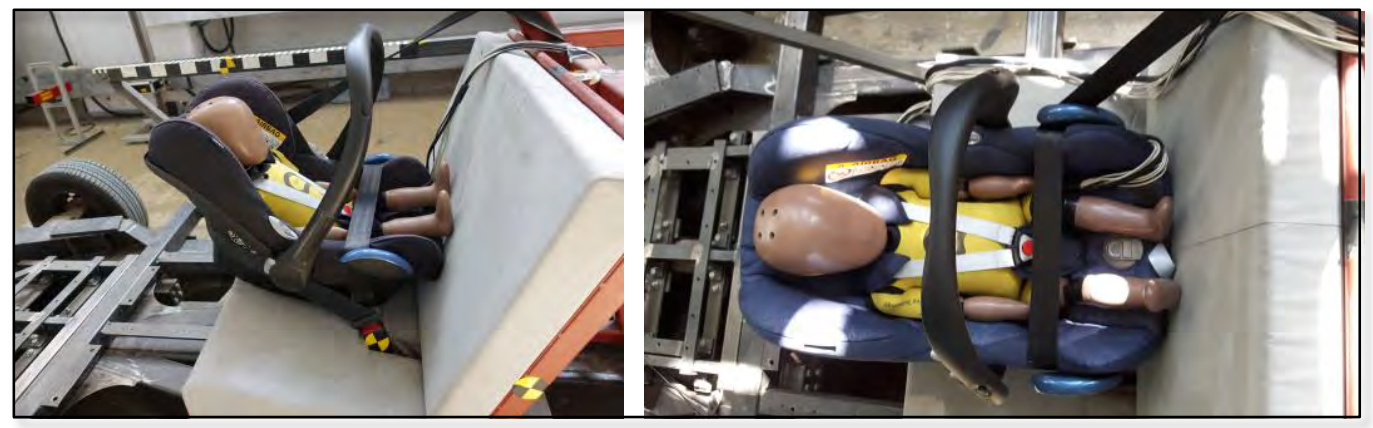

Abbildung 77: Vergleich der Befestigung der Babyschale Links: richtige Gurtführung (Versuch 1); Rechts: falsche Gurtführung (Versuch 2)

Dieser Sicherungsfehler führt im Ergebnis dazu, dass die Babyschale samt dem darinsitzenden Kind de facto nicht im Fahrzeug gesichert ist. Beide fliegen im Fall eines Frontalaufpralls ungesichert nach vorn und werden je nach Einbauposition erst durch das Armaturenbrett oder durch die Lehne des vorderen Sitzes gebremst. Diese Verzögerung ist dann entsprechend hoch und führt zu hohen Belastungen des Kindes. Im schlimmsten Fall wird der Kopf direkt durch harte Fahrzeugteile getroffen.

Zur Veranschaulichung der Folgen dieser Fehlbenutzung wurden zwei Schlittenversuche durchgeführt, die die richtige und die falsche Nutzung des Fahrzeuggurts gegenüberstellen. Die Nutzung eines Schlittens hat gegenüber der Verwendung eines kompletten Fahrzeugs vor allem den Vorteil, dass die Kinematik der Babyschale sehr gut zu erkennen und zu filmen ist. Ein harter Aufschlag der Babyschale findet nicht statt, dafür ist aber die deutliche Vorverlagerung zu erkennen und deren Folgen absehbar. Damit es zu keinen Schäden am Dummy kommt, wurde die Babyschale im Misuseversuch derart gesichert, dass sie sich zwar zunächst ungehindert vorverlagern kann, ab einem bestimmten Punkt jedoch durch einen Sicherungsgurt gehalten wird.

Für den Versuch wurde ein Q1-Dummy verwendet. Dieser entspricht in Größe und Gewicht dem Durchschnitt eines einjährigen Kindes. 
Der Versuch wurde in Anlehnung an die ECE-R 129 durchgeführt. Entsprechend wurden Sitzbank, Verzögerungskorridor und Versuchskonfiguration gewählt. Die Verzögerungsverläufe beider Versuche lagen im vorgegebenen Korridor (s. Abbildung 78).

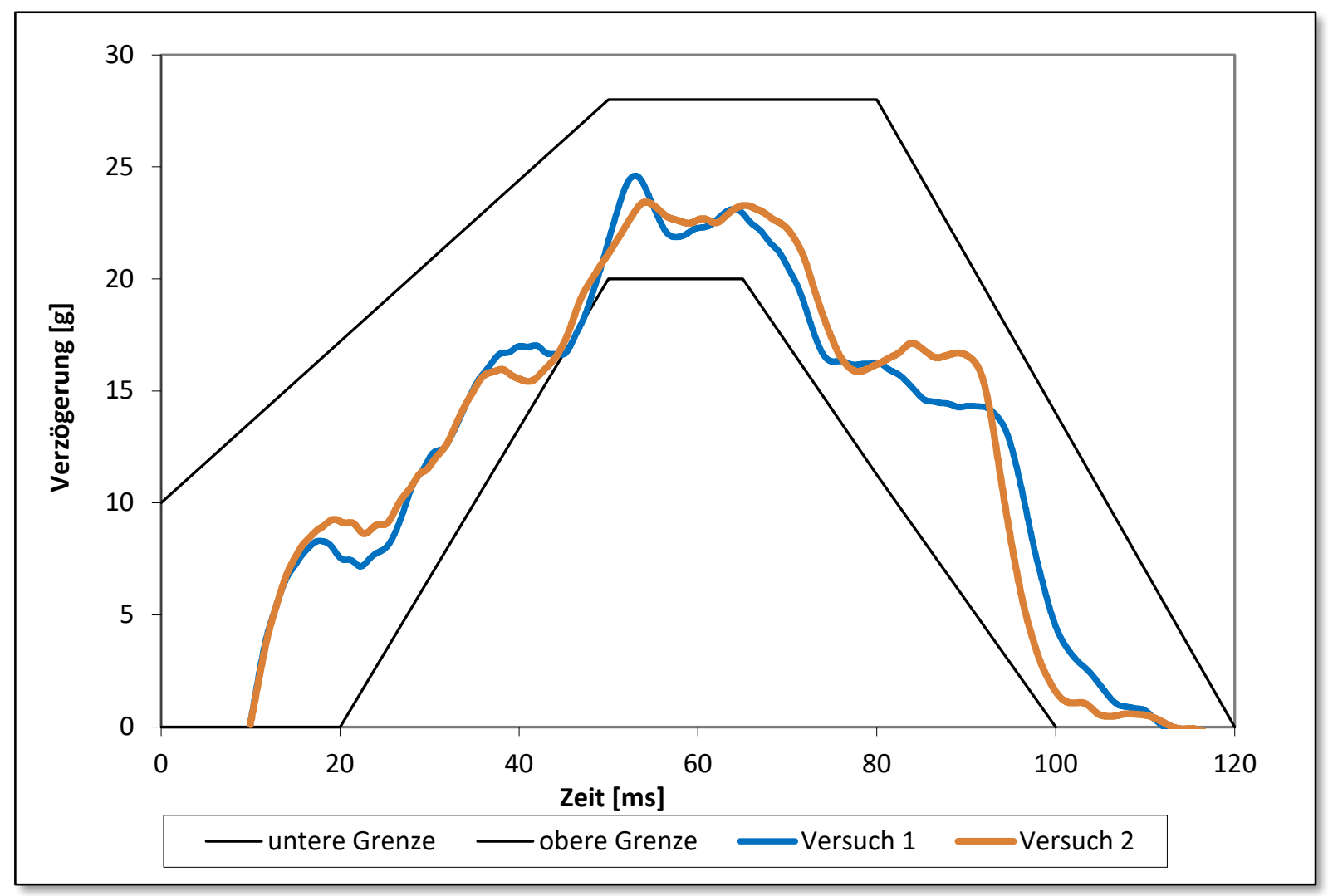

Abbildung 78: Zeitlicher Verzögerungsverlauf beider Vergleichsversuche

Im Vergleich beider Versuche ist erwartungsgemäß zu erkennen, dass sich die Babyschale im ersten Versuch (richtige Benutzung) moderat vorverlagert, dabei verformt wird und somit Energie aufnimmt. Die dabei erfassten biomechanischen Belastungswerte sind moderat und unterhalb der gesetzlich festgelegten Grenzwerte. Im zweiten Versuch mit falscher Gurtnutzung ist zu erkennen, dass sich die Babyschale nahezu ungehindert nach vorn bewegt. Die Vorverlagerung wird erst durch die zusätzliche Gurtsicherung der Schale gestoppt. Im realen Fahrzeug hätte es hier bereits einen harten Kontakt mit dem Innenraum gegeben. Die gemessenen Belastungswerte sind insgesamt ebenfalls moderat, wobei die Kopfbelastungen deutlich über denen vom Versuch 1 liegen. Die insgesamt niedrigen Belastungswerte sind dadurch begründet, dass die Schale durch eine zusätzliche, ebenfalls elastische Sicherung zurückgehalten wird. Ein Kontakt im Fahrzeuginnenraum wäre deutlich massiver. Abbildung 79 zeigt die Kinematik beider Versuche im Vergleich, in Tabelle 16 sind 
die gemessenen Belastungswerte beider Versuche und die gesetzlichen Grenzwerte dargestellt.

Die in diesem Vergleichsversuch dargestellte Fehlbenutzung zeigt beispielhaft, welche massiven Folgen bereits scheinbar kleine Fehler haben können, die in der Feldstudie häufig beobachtet wurden. Das bestätigt zwei, in diesem Bericht mehrfach geäußerte Erkenntnisse: Es besteht nach wie vor eine große Notwendigkeit zur Aufklärungsarbeit hinsichtlich der richtigen Nutzung von KSS und die Verbreitung von ISOFIX-Systemen, welches den dargestellten Fehler unmöglich macht, sollte vorangetrieben werden.

\begin{tabular}{|l|c|c|c|}
\hline \multicolumn{1}{|c|}{ Belastungsgröße } & $\begin{array}{c}\text { Grenzwert } \\
\text { gemäß } \\
\text { ECE-R 129 }\end{array}$ & $\begin{array}{c}\text { Messwerte } \\
\text { Versuch 1 }\end{array}$ & $\begin{array}{c}\text { Messwerte } \\
\text { Versuch 2 }\end{array}$ \\
\hline HIC & 600 & 163 & 619 \\
\hline HIC 15 & 75 & 146 & 530 \\
\hline HIC 36 & & 31 & 56 \\
\hline Kopf $\mathrm{a}_{3 \mathrm{~ms}}[\mathrm{~g}]$ & & 0,15 & 0,84 \\
\hline Obere Halskraft $\mathrm{F}_{\mathrm{z}}[\mathrm{kN}]$ & 55 & 15 & 38 \\
\hline Oberes Halsmoment $\mathrm{M}_{\mathrm{y}}[\mathrm{Nm}]$ & & 44 & 31 \\
\hline Brust $\mathrm{a}_{3 \mathrm{~ms}}[\mathrm{~g}]$ & & & \\
\hline Becken $\mathrm{a}_{3 \mathrm{~ms}}[\mathrm{~g}]$ & & & \\
\hline
\end{tabular}

Tabelle 16: Gemessene Belastungswerte im Vergleich zu gesetzlichen Grenzwerten für Q1-Dummy 


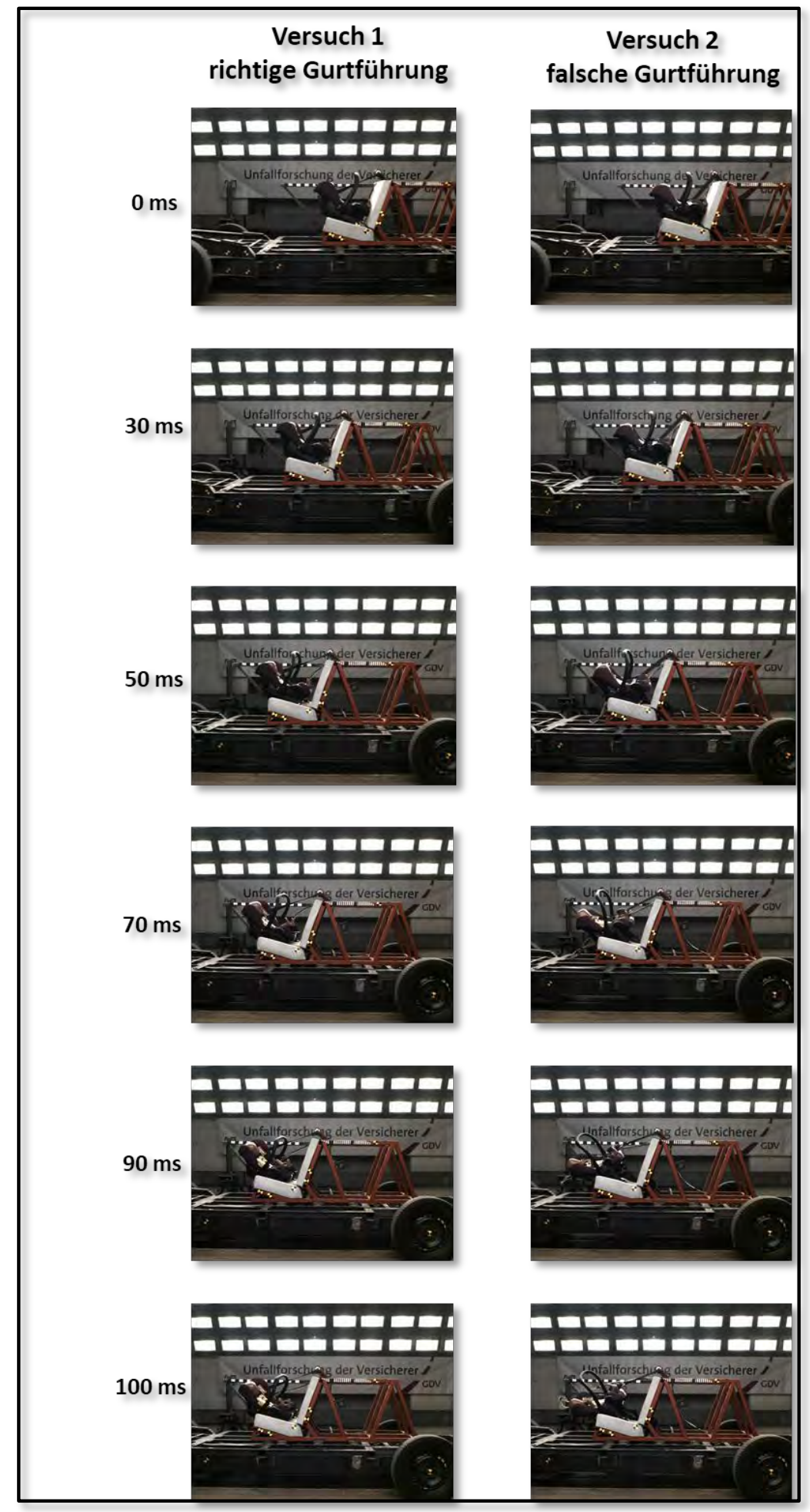

Abbildung 79: Vergleich der Kinematik beider Sitzschalen zu verschiedenen Zeitpunkten 


\section{GDV \\ DIE DEUTSCHEN VERSICHERER}

Gesamtverband der Deutschen Versicherungswirtschaft e. V.

Wilhelmstraße 43 / 43G, 10117 Berlin

Postfach 0802 64, 10002 Berlin

Telefon 030 / 2020 - 50 00, Fax 030 / 2020 - 6000

Internet: www.gdv.de, www.udv.de 